\title{
Simulationsrechnungen anisoplanatischer Übertragungsfunktionen für solare Adaptive Optik
}

\author{
Dissertation \\ zur Erlangung des Doktorgrades \\ der Mathematisch-Naturwissenschaftlichen Fakultäten \\ der Georg-August-Universität zu Göttingen
}

vorgelegt von

Markus Josef Sailer

aus Kempten im Allgäu

Göttingen 2006 
D7

Referent: Prof. Dr. Franz Kneer

Korreferent: Prof. Dr. Oskar von der Lühe

Tag der mündlichen Prüfung: 


\section{Inhaltsverzeichnis}

Inhaltsverzeichnis 3

\begin{tabular}{ll}
\hline Kurzfassung & 7
\end{tabular}

\begin{tabular}{lll}
\hline & Einleitung & 9
\end{tabular}

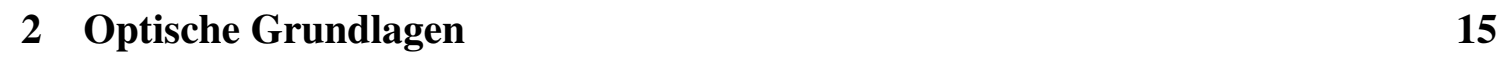

2.1 Lineare Systeme. . . . . . . . . . . . . . . . . . . . . . . . 15

2.2 Wellenoptik $\ldots \ldots \ldots \ldots \ldots \ldots \ldots \ldots \ldots$

$2.2 .1 \quad$ Fresnel'sche Näherung . . . . . . . . . . . . . . . . . 17

2.2 .2 Fraunhofer'sche Näherung . . . . . . . . . . . . . . . . . . . . 18

2.3 Zur Bildentstehung . . . . . . . . . . . . . . . . . . . . . . . . . 19

2.4 Aberrationsfreies optisches System $\ldots \ldots \ldots \ldots \ldots \ldots \ldots$

3 Turbulenz der Erdatmosphäre 23

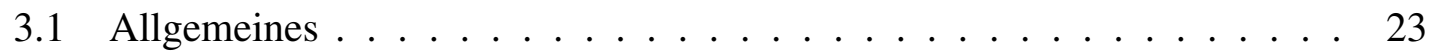

3.2 Mechanik der Turbulenz $\ldots \ldots \ldots \ldots \ldots \ldots$

3.2 .1 Ursachen . . . . . . . . . . . . . . . . . . . . 23

3.2 .2 Strömungsmechanische Größen . . . . . . . . . . . . . . . . 24

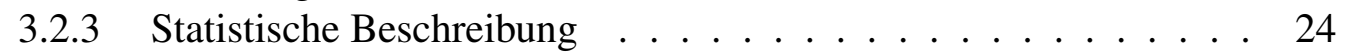

3.2 .4 Kolomogorov'sches Modell . . . . . . . . . . . . . . . . . . 24

3.2 .5 Turbulente Brechungsindexvariationen . . . . . . . . . . . 26

$3.2 .6 \quad C_{n}^{2}$-Höhenprofil . . . . . . . . . . . . . . . . . . . . . 27

3.3 Momente und Kohärenz. . . . . . . . . . . . . . . . . . . . . . . . . . . 28

3.4 Aberration durch Turbulenz . . . . . . . . . . . . . . . . . . . . . . . . . . . 29

$3.4 .1 \quad$ Inkohärente Übertragungsfunktion . . . . . . . . . . . . . . . . . 30

3.4 .2 Langzeit- und Kurzzeitbelichtung . . . . . . . . . . . . . . . 32

3.4 .3 Bildschärfe - Strehl'sche Definitionshelligkeit . . . . . . . . . . 32

$3.4 .4 \quad$ Modale Repräsentation von Atmosphärischen Störungen . . . . . 33

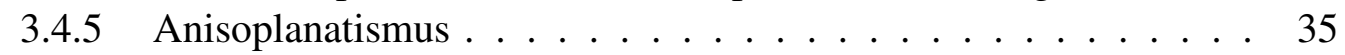

$3.4 .6 \quad$ Zeitliche Effekte - Greenwood-Frequenz $\ldots \ldots \ldots \ldots$

4 Adaptive Optik 41

$4.1 \quad$ Funktionsweise einer Adaptiven Optik . . . . . . . . . . . . . . . . . 41

$4.1 .1 \quad$ Allgemeiner Aufbau und wichtigste Elemente . . . . . . . . . . 41

4.1 .2 Wellenfrontmessung - WFS . . . . . . . . . . . . 43

4.1.2.1 Shack-Hartmann-Wellenfrontsensor . . . . . . . . . 43 
$4.1 .2 .2 \quad$ Messgenauigkeit . . . . . . . . . . . . 45

4.1 .2 .3 Rauschen . . . . . . . . . . . . . . . . . . . . . . . . . . . . . . . .

4.1 .3 Wellenfrontrekonstruktion . . . . . . . . . . . . . . 46

4.1.3.1 Zonaler und Modaler Ansatz . . . . . . . . . . . . . 47

4.1.3.2 Lösung des inversen Problems . . . . . . . . . . . . . . . 48

4.1 .4 Wellenfrontkorrektur . . . . . . . . . . . . . . . . . . . . 49

4.1.4.1 Bimorphe Spiegel . . . . . . . . . . . . . 50

\begin{tabular}{lll|}
\hline & AO-Simulation & $\mathbf{5 3}$
\end{tabular}

5.1 Überblick . . . . . . . . . . . . . . . . . . . . 53

$5.2 \quad$ Generierung von Phasenschirmen mit dem Programmpaket TurbuLenZ $\quad 53$

$5.2 .1 \quad$ Eingabe-Parameter . . . . . . . . . . . . 54

5.2 .2 Berechnung der Phasenschirme . . . . . . . . . . . . 55

5.2 .3 Modellierung der Dynamik . . . . . . . . . . . . . . . . 56

5.2 .3 .1 Wind . . . . . . . . . . . . . . . . . 56

5.2 .3 .2 Dekorrelation . . . . . . . . . . 56

5.2 .4 Wellenfrontpropagation . . . . . . . . . . . . . 57

5.3 Einfache Modellierung von KAOS . . . . . . . . . . . . . . . . . . 58

5.3 .1 Wellenfrontmessung . . . . . . . . . . . . . . . . . . . . . . . . 59

5.3 .2 Deformierbarer Spiegel . . . . . . . . . . . . . . . . . . 60 60

5.3 .3 Kalibration . . . . . . . . . . . . . . . . 62

5.3 .3 .1 Singular Value Decomposition . . . . . . . . . 62

5.3.3.2 Korrekturstärke und cutoff-Parameter . . . . . . . . . . 63

5.3.3.3 Gegenseitige Orientierung von WFS und DM . . . . . 64

5.3 .4 Wellenfrontkorrektur . . . . . . . . . . . . . . . 65

5.4 Output - Übertragungsfunktionen . . . . . . . . . . . . . . . . . . 66

5.4.1 PSF, Übertragungsfunktionen und Strehl-Verhältnis . . . . . . . . 67

5.4 .2 Parameterraum . . . . . . . . . . . . 68

\begin{tabular}{lll}
\hline & Beobachtungsmaterial & $\mathbf{7 1}$ \\
\hline
\end{tabular}

6.1 Instrumentelles . . . . . . . . . . . . . . . . . 71

$6.1 .1 \quad$ Das Vakuum-Turm-Teleskop . . . . . . . . . . . . . . 71

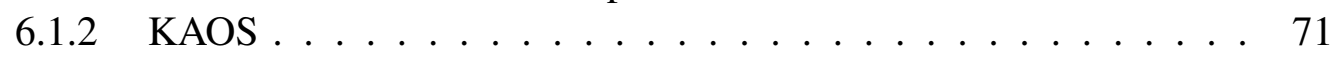

6.1 .3 DALSA Kamera . . . . . . . . . . . . . . . . 73

6.2 Beobachtungen am VTT $\ldots \ldots \ldots \ldots . \ldots \ldots$

6.2 .1 Speckle-Daten . . . . . . . . . . . . . . . 73

6.2 .2 AO-Mitschriften . . . . . . . . . . . . . . . . . . . . . . . . . . . . . . 74

6.3 Datenmaterial vom SST . . . . . . . . . . . . . . 76

\begin{tabular}{lll}
\hline 7 & Ergebnisse & $\mathbf{7 7}$
\end{tabular}

$7.1 \quad$ Parameterstudien zu Simulationsrechnungen . . . . . . . . . . . . . . . . 77

7.1 .1 Seeing und Korrekturgrad $\ldots \ldots \ldots 77$

7.1 .2 Winkelabhängigkeit $\ldots \ldots \ldots$. . . . . . . . . . . . . . . . . . . . . 85

7.1 .3 Dekorrelation durch Wind . . . . . . . . . . . . . . . . . . . . 89

7.2 Rekonstruktion von AO-Speckle-Daten . . . . . . . . . . . . . . . . 93

7.2.1 Modifikation des Programmpakets . . . . . . . . . . . . . . 96 
7.2.1.1 Bestimmung des Lockpunktes . . . . . . . . . . . . . . 96

7.2.1.2 Modellierung der Richtungsanisotropie . . . . . . . . . 97

7.2 .2 Drei Näherungen . . . . . . . . . . . . . . . . . . . . . 99

7.2.2.1 Ansatz A und B - Korff'sche STFs . . . . . . . . . . . 99

7.2.2.2 Ansatz C - Simulation der AO-Korrektur . . . . . . . . 101

7.2 .3 Unterschiede in den Rekonstruktionen . . . . . . . . . . . . 105

$7.2 .3 .1 \quad \mathrm{~B}-\mathrm{A} \ldots \ldots \ldots \ldots 10 \ldots \ldots$

$7.2 .3 .2 \quad \mathrm{C}-\mathrm{A} \ldots \ldots \ldots \ldots \ldots$

$7.2 .3 .3 \quad \mathrm{C}-\mathrm{B} \quad \ldots \ldots \ldots$

7.2 .4 Winkelabhängigkeit des Kontrastes . . . . . . . . . . . . . . 109

7.2 .5 Weitere Anmerkungen zur Analyse . . . . . . . . . . . . . . . 111

7.3 Beobachtungsgestützte Simulation . . . . . . . . . . . . . . . . . 112

7.3.1 Rekonstruktionsmatrizen und Fingerabdruck der Geometrie . . . 112

7.3 .2 Parameter aus der AO-Aktivität . . . . . . . . . . . . . . . 112

7.3 .3 Anisoplanatische PSFs aus den Speckle-Daten . . . . . . . . . . 114

7.3.4 Simulationsgestützte Beobachtung . . . . . . . . . . . . . . 115

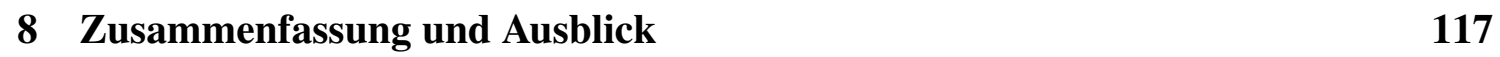

\begin{tabular}{lr}
\hline A Fouriertransformation & 119
\end{tabular}

\begin{tabular}{|rr}
\hline B Der Satz von Wiener-Khinchine & 123
\end{tabular}

\begin{tabular}{ll}
\hline Literaturverzeichnis & 125
\end{tabular}

\begin{tabular}{ll}
\hline Danksagung & 131
\end{tabular}

\begin{tabular}{ll}
\hline Lebenslauf & 135
\end{tabular} 



\section{Kurzfassung}

Mit dem Adaptiven Optischen (AO) System KAOS, installiert am Vakuum-Turm-Teleskop (VTT) auf Teneriffa (Spanien) und entwickelt am Kiepenheuer-Institut für Sonnenphysik, sind seit wenigen Jahren Beobachtungen mit Online-Korrektur des atmosphärischen Seeings möglich. Somit können bedeutend häufiger Rohdaten höchster Qualität aufgenommen werden, als dies ohne die AO möglich war, jedoch ergeben sich Probleme für die Verfahren zur Nachbehandlung von Bilddaten, wie auch für die wissenschaftliche Interpretation des quantitativen Informationsgehaltes solcher Daten. Der Grund dafür ist vor allem in der gegenüber dem rein atmosphärischen Einfluss veränderten Statistik der residuellen Wellenfrontfehler zu suchen, die nach der AO-Korrektur übrigbleiben. Dabei hängt die Größe der Unterschiede und die Stärke der Effekte von der Korrekturempfindlichkeit des AO-Systems ab, das sich seinerseits auf die atmosphärischen Bedingungen einstellt und damit zeitabhängig wird. Durch die Korrektur der Wellenfront in einer einzigen Richtung tritt zusätzlich als auffälliger Effekt der Anisoplanatismus in Erscheinung, der die Abbildungsqualität blickrichtungsabhängig macht. Auch die zeitliche Verzögerung zwischen Messung und Korrektur der Wellenfront beeinflusst die Güte der Kompensationsleistung.

Aus dem Wunsch heraus, diese Punktverbreiterungsfunktion (PSF) bzw. die äquivalente Optische Übertragungsfunktion (OTF) zu modellieren, um Aussagen über die Wirkung von KAOS auf die Bildqualität treffen zu können, wurde in dieser Arbeit der Weg einer Simulation gewählt. Darin wurden Wellenfronten als synthetische Phasenschirme mit atmosphärischer Statistik der Phasenverzögerungen durch den optischen Aufbau propagiert, wobei die wichtigsten optischen Elemente des AO-Systems modelliert wurden, um eine realistische Phasenkompensation der Wellenfront zu erreichen. Aus jeweils einer Reihe so korrigierter Phasenschirme, d. h. geglätteten Wellenfronten, wurden für eine Reihe von Kombinationen der Parameter (Blickwinkel, Korrekturniveau, Turbulenzstärke, Wind) die entsprechende Langzeit-PSF und Speckle-Übertragungsfunktion (STF) berechnet und die Auswirkungen auf die Bildqualität untersucht.

Die Simulationsergebnisse wurden im Göttinger Speckle-Masking-Rekonstruktionsalgorithmus auf Beobachtungsdaten angewandt, wobei die konventionelle Annahme einer über das Gesichtsfeld konstanten PSF fallengelassen werden konnte. Hier zeigt der Verleich mit der herkömmlichen Methode signifikante Unterschiede in den rekonstruierten Intensitäten und im Kontrast. 



\section{Einleitung}

Nach der Erfindung des Teleskops und den detaillierten Zeichnungen von astronomischen Objekten, wie der Mondoberfläche oder der Monde des Planeten Jupiter, wurde Astronomen schnell klar, dass größere Teleskope besser waren was Empfindlichkeit und Auflösungsvermögen betrifft. Bis zur Mitte des zwanzigsten Jahrhunderts konnte jedoch nur die eine Seite verbessert werden. Die erreichbare räumliche Auflösung wird nämlich schon begrenzt, bevor das Licht in ein Teleskop am Erdboden fällt. Das Problem sind Schwankungen im Brechungsindex der Luft entlang der Sichtlinie zum astronomischen Objekt. Im Bild der Strahlenoptik stelle man sich ein praktisch paralleles Lichtstrahlenbündel vor, das die Atmosphäre der Erde erreicht, und das letztlich in das Teleskop gelangen wird. Nun durchquert davon jeder Lichtstrahl die Luftschichten entlang eines eigenen Weges und durchtritt unterschiedliche Turbulenzzellen mit unterschiedlichem Brechungsindex. Jedes Teilbündel wird dadurch von seiner ursprünglichen Richtung leicht abgelenkt und die in das Teleskop tretenden Lichtstrahlen sind nicht mehr parallel. Dies hat zur Folge, dass das Licht nicht mehr auf einen Bildpunkt fokussiert werden kann und nur ein unscharfes Bild erzeugt wird. Die Brechungsindexänderungen werden durch Dichteänderungen in Folge von ungleichmäßiger Erwärmung verursacht und prägen einem hindurchtretenden Lichtbündel den chaotischen Charakter der Luftturbulenzen auf. Dieses als 'Seeing' bezeichnete Phänomen wird schon von Isaac Newton 1704 beschrieben und verhindert die Beobachtung von feineren Details, als sie schon durch ein etwa $10 \mathrm{~cm}$ durchmessendes Teleskop möglich ist, selbst bei Teleskopen wie den vier Hauptteleskopen des VLT (je 8,2 m) am Paranal in Chile oder etwa den beiden segmentierten Keck-Teleskopen (je $10 \mathrm{~m}$ ). Eine Korrektur des Seeings war zunächst nicht denkbar, da die Störungen äußerst schnell fluktuieren (Zeitskala $\sim 10 \mathrm{~ms}$ ) und Konzepte, theoretische Grundlagen zur Lichtausbreitung und Messungen in der Atmosphäre noch lange nicht verfügbar waren. Fortschritte in Richtung größerer Auflösung wurden erst Mitte des 20. Jahrhunderts gemacht, als die erwähnten Voraussetzungen allmählich komplett waren und Ideen zur Entwicklung der sogenannten Adaptiven Optik (AO) geboren wurden.

\section{Historische Eckdaten}

Die Anfänge der Bemühungen zur Kompensation des atmosphärisch verursachten Seeings für astronomische Beobachtungen reichen zurück in das Jahr 1953 und beginnen mit der Publikation von H. Babcock (1953). Dort wurde zum ersten Mal ein Konzept für eine AO beschrieben: In einem dünnen Ölfilm ('Eidophor') sollten auf einem Spiegel über Bestrahlung mit einem Elektronenstrahl mechanische Spannung aufbaut werden, die die Dicke der Ölschicht und damit seine Oberflächenform lokal verändern sollten. Über die veränderte Lichtbrechungsgeometrie hätte man somit kontrollierten Einfluss auf die Rich- 
tung der reflektierten Lichtstrahlen bzw. äquivalent dazu auf die Deformation der Wellenfront und damit auf die Fokussierbarkeit des gesamten Lichtbündels. Zunächst konzentrierten sich die Bemühungen aber lediglich auf die niedrigste Ordnung der Korrektur, der Beruhigung der globalen Bildbewegung. Sie kann durch passende Verkippung eines Planspiegels (Tip/Tilt-Spiegel) erreicht werden, muss jedoch mit ausreichend hoher Frequenz durchgeführt werden. Korrekturen höherer Ordnung erforderten elektronische und mechanische Technologien, die damals noch nicht entwickelt waren.

Die theoretische Basis zur quantitativen Bestimmung der Begrenzung des optischen Auflösungsvermögens durch unkompensiertes atmosphärisches Seeing wurde in den 1960er und 70er Jahren von D. Fried, V. Tatarski und anderen gelegt. Viele Methoden wurden damals zur Korrektur nach der tatsächlichen Aufnahme vorgeschlagen, d. h. die Gewinnung fast beugungsbegrenzter Bilder aus der Verarbeitung von Beobachtungen, die durch den Einfluss der Turbulenz verzerrt wurden und ohne aktive Korrekturen dieser Effekte aufgenommen wurden. Hier sind nicht-AO-Methoden wie die von Labeyrie (1970), von Knox \& Thompson (1974) und Weigelt (1977) auch für die vorliegende Arbeit von grundlegender Bedeutung. Die erste Implementierung eines AO-Sytems mit Phasenkompensation, so wie sie heute in Gebrauch ist, wurde 1973 unter J. Hardy für das 1,6-m-Teleskop am ARPA ${ }^{1}$ Maui Optical Site (AMOS) entwickelt, als Methode für die außerordentlich verbesserte Abbildung von 'Weltraum-Objekten'. Heraus kam ein 21-Kanal-System, das auf der stufenweisen Messung von Phasendifferenzen beruhte. Für die Rekonstruktion der Wellenfront (WF) benutzte man eine Matrixanordnung solcher Messzellen, die das Bild der gesamten Eintrittspupille des Teleskops abdeckte. Der Erfolg der Experimente fürte zur Etablierung dieser Technik der Wellenfrontkompensation. Danach wurde Mitte der 70er Jahre das theoretische Fundament für die späteren technischen Entwicklungenen durch Arbeiten zur Wellenfrontrekonstruktion und AO-Leistungsfähigkeit von R. Hutchin (vorher Hudgin), D. Fried und D. Greenwood gelegt. In den 70er Jahren waren zwar schon nicht-öffentliche Forschungen und Entwicklungen auf experimentellem Gebiet schon weiter, unter anderem im Rahmen des amerikanischen SDI-Programms, jedoch wurden die meisten der bis dahin geheimen Forschungsergebnisse der Öffentlichkeit erst preisgegeben, als auch Astronomen Laserleitsterne für die AO vorschlugen und außerdem der Kalte Krieg zu Ende ging. So wurde erst Anfang der 90er Jahre das erste öffentlich zugängliche astronomische AO-System ('COME-ON') am 3,6-m-Teleskop des European Southern Observatory (ESO) auf La Silla (Chile) in Betrieb genommen. Seit dieser Zeit hat sich jedoch viel getan und die AO hat den Durchbruch zu einer populären, sich immer schneller verbreitenden Technologie geschafft. Mittlerweile sind durch billige Rechenresourcen einfache Systeme so erschwinglich geworden, dass die Zahl der Teleskope mit AO-Unterstützung unübersehbar geworden ist.

\section{AO in der Sonnenbeobachtung}

Die Sonnenbeobachtung hat, obwohl sie im 'Medienrummel' der Großteleskope der NachtAstronomie oft übersehen wird, immer wieder entscheidende Beiträge zur Entwicklung der Adaptiven Optik gegeben! Dabei sind Acton \& Smithson (1992) zu nennen, die mit einem segmentierten adaptiven Spielgel und einer solaren Pore als Referenzobjekt eine stabile AO-Korrektur erreichten. Dies bedeutete einen großen Fortschritt, da solare

\footnotetext{
${ }^{1}$ Advanced Research Projects Agency
} 
Adaptive Optik bedeutend mehr Rechenleistung erfordert, als der Betrieb einer AO bei Nacht. Da hochauflösende Beobachtungen der Sonnenoberfläche nur bei Abbildung kleiner Ausschnitte der Sonnenscheibe auf den Detektor möglich sind, hat man keine richtige Punkt-Lichtquelle zur Verfügung, deren ideales Beugungsmuster bekannt wäre und über den Vergleich mit der tatsächlich gemessenen Form direkt eine Entfaltung des Bildes ermöglichte. Zudem sind keine statischen Strukturen auf der Sonnenoberfläche zu sehen, vielmehr hat man es mit einer Entwicklung der Strukturen auf der Zeitskala von Minuten zu tun. Dazu kommt das entschieden schlechtere Seeing bei Tage, da die Sonneneinstrahlung viel stärkere Temperaturunterschiede in der Atmosphäre und am Boden bewirkt, der dann wie eine heiße Kochtopfplatte wirkt und zusätzliche Turbulenzen durch die konvektive Bewegung der erwärmten Luft erzeugt.

Als erstes Sonnenteleskop der zivilen Forschung wurde 1989 das 76-cm Dunn Solar Telescope (DST) am U.S. National Solar Observatory (NSO) auf dem Sacramento Peak, New Mexico, mit einem AO-System ausgestattet, das zuletzt 2002 modernisiert wurde und durch Erhöhung der Freiheitsgrade bzw. Spiegelaktuatoren auf 97 zum derzeit bestkorrigierenden System avancierte.

Im Jahr 1999 wurde das Swedish Vakuum Solar Telescope (SVST) auf La Palma, Spanien, mit einer AO aufgerüstet, die beim Wechsel zum 1-Meter Swedish Solar Telescope (SST) 2002 mit dem Ziel in Betrieb genommen wurde, die Beugungsgrenze für die Auflösung ( 0 '” 1 für blaues Licht) am SST zu ermöglichen, was mit Hilfe einer 'Low-order' AO (37 Aktuatoren) auch gelang. Es ist derzeit die wohl führende Einrichtung in Sachen hochauflösende Beobachtung.

Hochauflösende Beobachtungen werden regelmäßig auch am vom Kiepenheuer-Institut für Sonnenphysik (KIS) betriebenen Vakuum-Turm-Teleskop (VTT) auf Teneriffa (Spanien) aufgenommen, wobei das Adaptive Optische System KAOS mit einem 35-AktuatorenSpiegel fest installiert und für alle Beobachtungen verwendbar ist. Auf dieses AO-System wird in der Arbeit noch Bezug genommen. Die in diesem Zusammenhang besondere Stellung des VTTs wird dadurch begründet, dass dort 2003 der Regelkreis einer Erweiterung von KAOS zur sogenannten Multi-konjugierten Adaptiven Optik (MCAO) geschlossen wurde. Mit der MCAO kann die Wellenfront gleichzeitig in zwei verschiedenen atmosphärischen Schichten korrigiert werden, sodass sich der korrigierte Ausschnitt in den Beobachtungsdaten vergrößert.

Aus aktuellem Anlass will ich hier natürlich noch die anstehende Inbetriebnahme des neuen 1,5-Meter Sonnenteleskops GREGOR, ebenfalls auf Teneriffa, ansprechen. Es ist das Nachfolgeteleskop des früher von der Göttinger Sternwarte betriebenen Gregory-CoudéTeleskops. Die dort zunächst geplante Version des AO-Systems GAOS mit 60 Freiheitsgraden und einer Bandbreite der Regelschleife von $65 \mathrm{~Hz}$ ist eine Weiterentwicklung von KAOS.

\section{Numerischer Zugang}

Simulationen spielen im Zusammenhang mit der Untersuchung der Leistung Adaptiver Optischer Systeme eine immer bedeutendere Rolle. Sie ermöglichen schon im Vorfeld der Entwicklung eines tatsächlichen Systems die Bestimmung optimaler System-Parameter und die Ermittlung der effizientesten Kombinationen von Bauteilen. Unterschiedliche Algorithmen, die in ihrer Leistung von äußeren Bedingungen abhängen, wie etwa die 
Bewertung verschiedener Ansätze zur Wellenfrontmessung von der Art des beobachteten Objektes und der Stärke des Seeings abhängt, können in einer kontrollierten Weise und unter einer Vielzahl von Bedingungen getestet werden. Bezüglich der Bildentstehung nimmt beispielsweise die Simulation der Effekte statischer und dynamischer atmosphärischer Turbulenz auf die Wellenfronten eine zentrale Position ein und wird deswegen in dieser Arbeit näher untersucht werden. Ein Vorteil liegt hier darin, dass eine quasi beliebige Zahl von Parameter zur Modellierung der Situation herangezogen werden kann, was in einem analytischen Ansatz aufgrund der Komplexität und daraus resultierenden rechnerischen Schwierigkeiten scheitern muss. Daneben sind nicht nur statistisch gemittelte Größen einer weiteren Analyse zugänglich, sondern auch die konkreten 'atmosphärischen' Wellenfronten. Mit ihnen lassen sich etwa Abschätzungsalgorithmen prüfen, die mit einem Wellenfrontsensor gemessen werden, und aus weiteren Messungen des Systems Rückschlüsse auf die ursprünglichen atmosphärischen Verhältnisse ziehen sollen.

\section{Motivation und Ziel der Arbeit}

Am Vakuum-Turm-Teleskop (VTT), das vom Kiepenheuer-Institut für Sonnenphysik auf Teneriffa (Spanien) betrieben wird, bietet sich seit wenigen Jahren die Möglichkeit, Beobachtungen mit Hilfe des Adaptiven Optischen Systems KAOS zu gewinnen, das die Produktivität hinsichtlich der Gewinnung exzellenter Daten enorm erhöht hat. Die wissenschaftliche Analyse und Weiterverwendung dieser Daten macht jedoch bei der Nachbearbeitung Schwierigkeiten, da aufgrund des Anisoplanatismus eine ortsabhängige und darüber hinaus auch zeitabhängige Punktverbreiterungsfunktion (PSF) vorliegt. Neben allgemeinen Gesetzmäßigkeiten, die die Abhängigkeit der Bildqualität von den Parametern der Wellenfrontkorrektur theoretisch beschreiben, möchte man deshalb spezifischere Aussagen über die resultierende PSF bzw. deren Fouriertransformierte, die Optische Übertragungsfunktion OTF, gewinnen. Die Modellierung muss dazu auf das tatsächlich verwendete System KAOS zugeschnitten sein und hängt im konkreten Anwendungsfall von der tatsächlichen Korrekturaktivität der Module der Adaptiven Optik während der Datenerfassung ab.

Einen typischen Fall der post factum Nachbearbeitung stellt die weitere Bildrekonstruktion, etwa mit Methoden der Speckle-Interferometrie, dar. Man erreicht so eine weitere Verbesserung der räumlichen (oder Winkel-) Auflösung über die Leistungsfähigkeit der AO hinaus. Die Verlässlichkeit der rekonstruierten relativen Intensitäten bei der SpeckleBildrekonstruktion hängen jedoch empfindlich von der geschätzten wirklichen STF des kombinierten Systems aus Atmosphäre und optischem System ab, da das rekonstruierte Fourierspektrum der Daten im wesentlichen mit dem Kehrwert der Übertragungsfunktion multipliziert wird. Durch den Einsatz einer AO wird diese Funktion, verglichen mit dem rein atmosphärischen Fall, nicht mehr nur abhängig von einem einzigen, sondern einer ganzen Reihe weiterer Parameter. Die konventionelle Speckle-Übertragungsfunktion, die bisher in der solaren Speckle-Rekonstruktion gebräuchlich war, ist also nicht länger gültig. Aus dem Wunsch heraus, diese neuen OTFs bzw. die STFs zu verwenden, muss entweder das theoretische Modell modifiziert werden oder es müssen numerisch berechnete Übertragungsfunktionen bestimmt werden. In dieser Arbeit wurde der letztere Ansatz gewählt und der Weg einer Simulation eingeschlagen. In ihr wurden, soweit es die Bildentstehung betrifft, die wichtigsten optischen Elemente des Teleskops und sei- 
ner AO modelliert und die Propagation der Wellenfront mit simulierten atmosphärischen Distorsionen durch den spezifischen optischen Aufbau am VTT nachvollzogen. Aus den korrigierten synthetischen Phasenschirmen wurden die entsprechende Langzeit-PSF und Speckle-Übertragungsfunktion (STF) berechnet. Wie beabsichtigt, wurden Simulationsergebnisse im Göttinger Speckle-Masking-Rekonstruktionsalgorithmus auf Beobachtungen angewandt, wobei die Annahme einer über das Gesichtsfeld konstanten PSF fallengelassen werden konnte. Hier zeigten sich dann auch im Vergleich mit der herkömmlichen Methode die erwarteten signifikanten Unterschiede in den rekonstruierten Intensitäten und Kontrastwerten. Aus den berechneten Größen sind in Verbindung mit Daten aus dem laufenden Betrieb von KAOS, die die tasächliche technische Aktivität dokumentieren, weitere mögliche diagnostische 'Werkzeuge' zu entwickeln, die in der Praxis angewendet werden können!

Der Aufbau der Arbeit ist wie folgt: In den Kapiteln 2 bis 4 habe ich wichtige Grundlagen zusammengefasst, die für den Themenkreis der Arbeit und für das Verständnis der Begriffswelt, mit der dieses Forschungsgebiet arbeitet, von grundlegender Wichtigkeit sind. Dies betrifft erstens die Optik für die Bildentstehung. Zweitens ist einiges zum Einfluss der Atmosphäre auf die Lichtausbreitung zu sagen; hier kommt es mir auf die Ursachen der Wellenfrontdeformationen an. Drittens soll das Funktionsprinzip einer Adaptiven Optik erklärt werden.

Kapitel 5 behandelt dann überleitend die Simulation der AO, in der ich neben der Beschreibung der Phasenschirmgenerierung mit dem Programmpaket TURBULENZ auch auf mein eigenes Vorgehen bei der Berechnung der Übertragungsfunktionen eingehe.

Kapitel 6 beinhaltet eine kurze Sammlung wichtiger Angaben zum VTT und über experiementelle Daten, die dort von mir und von Kollegen am schwedischen Sonnenteleskop auf La Palma gewonnen wurden.

In Kapitel 7 gehe ich auf die Ergebnisse ein, die auf Rechnungen des Simulationscodes zur AO-Korrektur beruhen. Im ersten Teil verdeutlicht die Parameterstudie zu den Übertragunsgrößen und zur Bildqualität, welche Parameter in der Simulation berechnet werden können. Dies soll ein Gefühl dafür vermitteln, welche Abhängigkeiten den größten Einfluss auf die Modellierung haben. Der zweite Teil des Abschnittes geht auf die Anwendung der Rechnungen im Göttinger Speckle-Masking-Rekonstruktionscode ein und stellt eine (publizierte) Studie vor, die ich zusammen mit meinem Kollegen Dr. K. Puschmann hier am Institut für Astrophysik, Göttingen durchgeführt habe. Im Anschluss daran konnten Ideen und Gedanken zur Verwertung von Protokoll-Dateien zur AO-Aktivität während der Beobachtungen konnten nur kurz angerissen werden.

Schließlich blickt Kapitel 8 einerseits auf die Arbeit zurück, eröffnet aber andererseits Perspektiven für weitere Untersuchungen auf diesem hochinteressanten Forschungsgebiet. 



\section{Optische Grundlagen}

Zunächst sollen hier Grundlagen der optischen Abbildung im Rahmen der Theorie linearer Systeme gelegt werden, soweit sie für die in dieser Arbeit behandelten Problemkreise astronomischer Beobachtung relevant sind. Auch wenn die gegebenen Gleichungen nur gelegentlich explizit für die Analysen in dieser Arbeit benutzt werden, sind sie doch immer implizit. Somit stellen sie die Kulisse für alle optischen Aufbauten dar und verdienen deshalb wenigstens der Vollständigkeit halber eine kurze Behandlung.

\subsection{Lineare Systeme}

Um ein Verständnis des abbildenden Prozesses etwa in einem Teleskop zu gewinnen, beginnen wir hier mit dem allgemeinsten Formalismus. Wegen der Linearität der Wellengleichung, die die Propagation von elektromagnetischen Wellen beschreibt, kann der Bildentstehungsprozess als Abbildung vom Objektraum in den Bildraum angesehen werden. Die Einzelheiten dieser Abbildung spielen an diesem Punkt zunächst keine Rolle. Die dazu nötigen physikalischen Grundlagen werden in den folgenden Abschnitten eingeführt werden, jedoch ist die hier für uns wichtigste Eigenschaft der Abbildung ihre Linearität. In diesem Zusammenhang ist eine Abbildung $\mathcal{A}$ linear, wenn ihre Wirkung auf eine Summe von $N$ mehrdimensionalen Funktionen $g_{n}(\beta)$ mit Skalaren $b_{n}$ die Bedingung

$$
\mathcal{A}\left\{\sum_{n=1}^{N} b_{n} g_{n}(\beta)\right\}=\sum_{n=1}^{N} b_{n} \mathcal{A}\left\{g_{n}(\beta)\right\}
$$

erfüllt. Der Vorteil der Linearität ist im Grunde der, dass komplizierte Systeme mit dieser Eigenschaft in eine Reihe aus elementaren Objekten zerlegt werden können, die dann separat analysiert werden können. Betrachten wir ein allgemeines lineares System, das sich strikt deterministisch ohne dissipative Effekte entwickelt. Es kann durch

$$
f(\alpha)=\mathcal{A}\{g(\beta)\}
$$

beschrieben werden, wobei $g$ und $f$ die Eingabe und das Ergebnis darstellen. $\mathcal{A}$ ist ein linearer Operator, der die Abbildung von $g$ nach $f$ definiert. In der theoretischen Optik könnten die Funktionen $g$ und $f$ die Amplitudenverteilung in der Objektebene bzw. der Bildebene repräsentieren, wobei $\mathcal{A}$ die Physik der Lichtausbreitung beinhaltet. Man kann nun $g$ als eine Superposition von elementareren Bestandteilen ansehen, beispielsweise verschobene und gewichtete Delta-Funktionen

$$
g(\beta)=\int g(\mu) \delta(\beta-\mu) \mathrm{d} \mu,
$$


was nach Einsetzen in den vorhergehenden Ausdruck ergibt:

$$
f(\alpha)=\int g(\mu) \mathcal{A}\{\delta(\beta-\mu)\} \mathrm{d} \mu .
$$

Daher ist das Ergebnis der Abbildung durch eine Superposition von Impulsantworten $\mathcal{A}\{\delta(\beta-\mu)\}$ gegeben, die im Folgenden mit $h(\alpha, \beta)$ bezeichnet werden. Man findet, dass das Verhalten des Systems vollständig durch seine Impulsantwort charakterisiert wird. Eine wichtige Klasse von linearen Systemen, die eine erheblich vereinfachte Beschreibung bei der optischen Bildentstehung ermöglicht, ist die der isoplanatischen Operatoren, die invariant gegenüber räumlichen und zeitlichen Verschiebungen sind. Die Impulsantwort solcher Operatoren hängt nur von den relativen Koordinatenunterschieden zwischen Objekt- und Bildraum ab, d. h. $h(\alpha, \beta)=h(\alpha-\beta)$, und Gleichung 2.4 nimmt die Form einer Faltung an:

$$
f(\alpha)=\int g(\beta) h(\alpha-\beta) \mathrm{d} \beta=g * h .
$$

Ein immenser Vorteil wird offensichtlich, wenn man in den Fourierraum wechselt, wo die aufwändigen Berechnungen der Faltung durch eine einfache Multiplikation ersetzt werden.

\subsection{Wellenoptik}

Obwohl elektromagnetische Wellen Vektorcharakter besitzen, ableitbar aus den Maxwell'schen Gleichungen, kann man für eine adäquate Beschreibung der hier interessierenden Phänomene, z. B. die Ausbreitung durch ein turbulentes Medium oder die Beugung an der Teleskoppupille, getrost zu einer skalaren Theorie übergehen. Die Behandlung des Lichts als Welle wird beträchtlich vereinfacht, wenn die Kopplung zwischen elektrischen und magnetischen Vektoren vernachlässigt werden kann. Dies führt dann auf die skalare Beugungstheorie nach Fresnel und Kirchhoff, mit dem grundlegenden Ergebnis

$$
\begin{aligned}
U(P) & =\frac{A}{i \lambda} \iint_{\Sigma} \frac{e^{i k(r+s)}}{r s}\left[\frac{\cos (\boldsymbol{n}, \boldsymbol{r})-\cos (\boldsymbol{n}, \boldsymbol{s})}{2}\right] \mathrm{d} \Sigma \\
& =\frac{1}{i \lambda} \iint_{\Sigma} U_{1}\left(P_{1}\right) \frac{e^{i k s}}{s} \mathrm{~d} \Sigma,
\end{aligned}
$$

mit der Abkürzung

$$
U_{1}\left(P_{1}\right)=A \frac{e^{i k r}}{r}\left[\frac{\cos (\boldsymbol{n}, \boldsymbol{r})-\cos (\boldsymbol{n}, \boldsymbol{s})}{2}\right] .
$$

Dabei ist die Konstante $A$ ein Amplitudenfaktor, der durch die Feldstärke der Lichtquelle bestimmt ist. In Betrachtungen, die sich nicht mit absoluten Helligkeiten von Beobachtungsobjekten befassen, ist dieser Faktor unwichtig und kann ignoriert werden. Die komplexen Exponentialfunktionen stellen zwei Huygens'sche Elementarkugelwellen dar, die sich in Richtung der Vektoren $r$ und $s$ ausbreiten und dabei die Strecken $r=|\boldsymbol{r}|$ bzw. 


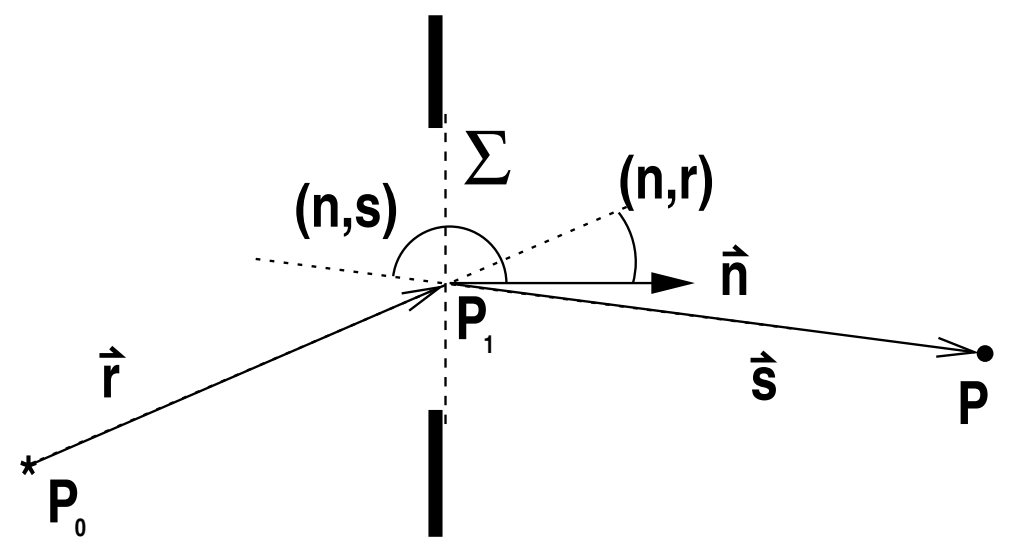

Abbildung 2.1: Zur Notation beim Kirchhoff-Fresnel-Integral: Beugung an einer Blende.

$s=|s|$ durchlaufen. Der Cosinus-Term, auch Inklinationsfaktor genannt, stellt einen Anisotropiefaktor für die Richtungsverteilung der Wellen dar. Wie in Abb. 2.1 skizziert, bringt das Beugungsintegral die Feldamplitude $U_{0}$ einer monochromatischen Lichtquelle der Wellenzahl $k=2 \pi / \lambda$ am Punkt $P_{0}$ in Beziehung mit dem Feld $U_{1}$ an der Stelle $P$, wobei das Lichtwellenfeld irgendwo dazwischen an einer Apertur $\Sigma$ gebeugt wird. Das resultierende Feld bei $P$ ist also gewissermaßen eine Überlagerung von Elementarwellen, die von den Punkten auf $\Sigma$ auszugehen scheinen, denen jedoch dort neben der Anisotropie (Inklinationsfaktor) auch noch der Amplituden- und Phasenfaktor $1 /(i \lambda)$ aufgeprägt wird.

Dieser Meilenstein in der Entwicklung der Theorie der Optik ist von unwahrscheinlicher Tragweite und Nützlichkeit. Die Herleitung kann in der Literatur, etwa in Born \& Wolf (1999), gefunden werden.

\subsubsection{Fresnel'sche Näherung}

Betrachtet man den allgemeinen Fall, wird das Beugungsintegral nichttrivial und Berechnungen werden sehr aufwändig. Deswegen werden an dieser Stelle üblicherweise einige Näherungen betrachtet, die, wenigstens für Anwendungen in der Astronomie, die Allgemeingültigkeit nur sehr schwach einschränken. Im astronomischen Umfeld wird die Lichtquelle eine sehr weit entfernte Konstellation bzw. Struktur sein und die beugende Öffnung wird aus der Erdatmosphäre und dem Teleskop bestehen. Diese Blende ist um viele Größenordnungen kleiner, als die Entfernung zur Lichtquelle, und somit sind im Integral nur sehr kleine Winkel $(\boldsymbol{n}, \boldsymbol{r})$ zu berücksichtigen und die paraxiale Näherung darf verwendet werden. Wir können also den Cosinus-Term in 2.7 durch 1 ersetzen und die Strecke $s$ durch die Distanz zwischen Blende und Bildebene $z$ ersetzen, bzw. in der Exponentialfunktion bis zur ersten Ordnung entwickeln. Setzen wir noch $A=1$, erhalten wir das Beugungsintegral in der Fresnel'schen Näherung:

$$
U(x, y, z)=\frac{e^{i k z}}{i \lambda z} \int_{-\infty}^{+\infty} \int_{1}\left(x_{1}, y_{1}\right) e^{i \frac{k}{2 z}\left(\left(x_{1}-x\right)^{2}+\left(y_{1}-y\right)^{2}\right)} \mathrm{d} x_{1} \mathrm{~d} y_{1} .
$$


Das Feld $U_{1}$ stellt in diesem Bild das einlaufende komplexe Feld $\frac{1}{r} e^{i k r}$ dar, multipliziert mit der Pupillentransmissionsfunktion. Der Index soll andeuten, das die Beschreibung dem Koordinatenraum $\boldsymbol{x}_{1}$ zugeordnet ist. Die zweidimensionalen Koordinaten $\left(x_{1}, y_{1}\right)$ bzw. $(x, y)$ bezeichnen also die Aperturebene bzw. die Bildebene. Die Fresnel'sche Näherung, auch Nahfeld-Näherung genannt, ist gültig unter den Bedingungen $x_{1}^{2}+y_{1}^{2} \ll z^{2}$ und $x^{2}+y^{2} \ll z^{2}$, die allerdings nicht stark einschränken. Gleichung (2.8) hat offenbar die Form einer Faltung (G1. 2.5)

$$
U(\boldsymbol{x})=U_{1}\left(\boldsymbol{x}_{\mathbf{1}}\right) * f_{z}\left(\boldsymbol{x}-\boldsymbol{x}_{\mathbf{1}}\right),
$$

für die wir die Funktion $f_{z}$ durch

$$
f_{z}(x, y, z) \equiv \frac{e^{i k z}}{i \lambda z} e^{i \frac{k}{2 z}\left(x^{2}+y^{2}\right)}
$$

definiert haben. Eine andere gebräuchliche und nützliche Sichtweise ist die Darstellung mit Hilfe einer Fouriertransformation, die in Gleichung 2.8 impliziert ist und nach Ausmultiplizieren des Exponenten in Erscheinung tritt. Allerdings bleibt ein multiplikativer Faktor, der von der Feldverteilung in der Pupillenebene abhängt:

$$
U(\boldsymbol{x})=f_{z}(\boldsymbol{x}) \cdot \mathcal{F}\left\{U_{1} e^{i \frac{k}{2 z}\left(x_{1}^{2}+y_{1}^{2}\right)}\right\} .
$$

Mit der zweidimensionalen Fouriertransformation $\mathcal{F}$ erhalten wir außerdem die zu $(x, y)$ konjugierten räumlichen Fourierfrequenzkoordinaten in der Fokalebene

$$
\left(\kappa_{x}, \kappa_{y}\right)=\left(\frac{x}{\lambda z}, \frac{y}{\lambda z}\right)
$$

mit der Dimension einer inversen Länge.

\subsubsection{Fraunhofer'sche Näherung}

Wenn die Detektorebene sehr weit von der beugenden Apertur entfernt ist, kann der Ausdruck 2.8 für die Fresnel'sche Beugung noch weiter vereinfacht werden. Wenn für die Entfernung zur beugenden Öffnung gilt $z \gg\left(x_{1}^{2}+y_{1}^{2}\right) k / 2$ nähert sich der Exponentialterm der Eins und das gebeugte Feld wird einfach

$$
U=f_{z} \cdot \mathcal{F}\left\{U_{1}\right\}
$$

Lassen wir nun die Punktquellenfunktion $f_{z}$ weg, denn sie ist nur ein multiplikativer Faktor, der Amplituden- und Phaseninformationen enthält. Dann zeigt sich, dass das Feld in der Bildebene proportional zur Fouriertransformierten der Feldkonfiguration in der Aperturebene wird. Darin besteht genau die Fraunhofer- oder Fernfeldnäherung. Für eine Blende von $1 \mathrm{~cm}$ Durchmesser, die von Licht der Wellenlänge $0.6 \mu \mathrm{m}$ bestrahlt wird, gilt die Frauhofer'sche Näherung für Abbildungen mit $z \gg 130 \mathrm{~m}$. Diese Einschränkung wiegt im Vergleich zur allgemeiner anwendbaren Nahfeldtheorie schwerer, hat aber den Vorteil der erheblich einfacheren Berechnung. Da große Distanzen zwischen Blende und Bildebene gefordert sind, wollen wir für den Augenblick den Fall $z \approx \infty$ betrachten. Parallele Lichtstrahlen einer astronomischen Quelle können durch eine positive Linse in einer 


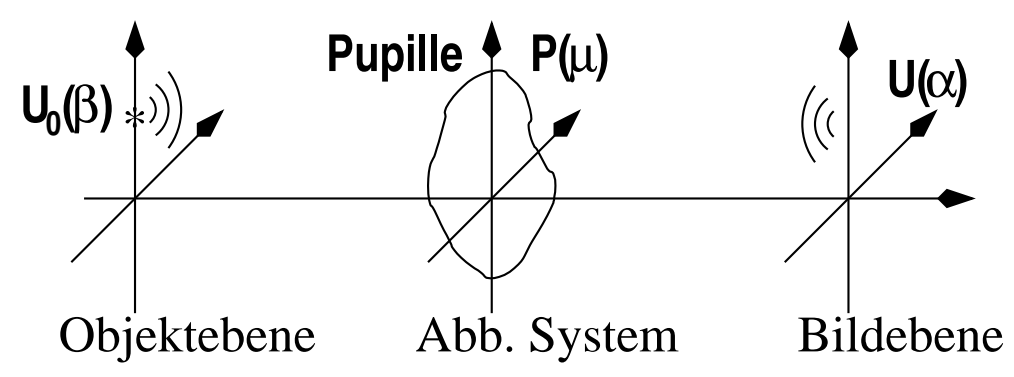

Abbildung 2.2: Ein allgemeines abbildendes System. Licht von der Quelle $U_{0}$ durchläuft ein beliebiges (lineares) optisches System, das ein fokussiertes Bild $U$ erzeugt. Alle Beugungseffekte des Systems werden mit seiner Pupille $P$ assoziiert.

im Endlichen liegenden Ebene fokussiert werden, so dass wir sagen können, die Linse bringe das Unendliche in die Fokusebene. Mit derselben Argumentation können alle abbildenden Systeme, die als Ergebnis fokussierte Strahlen liefern, mit der Fernfeldtheorie behandelt werden, ungeachtet der Details der Abbildung. Im Folgenden wird in den Analysen bisweilen Gebrauch von den dimensionslosen reduzierten Variablen

$$
\left.\left(s_{x}, s_{y}\right)=\left(\frac{x_{1}}{\lambda}, \frac{y_{1}}{\lambda}\right)\right) \quad \text { und } \quad\left(\alpha_{x}, \alpha_{y}\right)=\left(\frac{x}{z}, \frac{y}{z}\right)
$$

gemacht werden, mit der fetten Notation $s \rightleftharpoons \alpha$. Die $\alpha$ sind Richtungs-Cosinus der gebeugten Welle mit Einheiten in Radian, gültig für die Bildebene, die $s$ dagegen sind dazu konjugierte Winkelfrequenzen, die für die gebeugten wie auch die einlaufenden Wellen benutzt werden können und die räumlichen Koordinaten in der Ebene der beugenden Apertur benutzen. Die Einheiten der Winkelfrequenzen sind inverse Radian. Gehen wir in der Beschreibung also über von $U_{0}(\boldsymbol{\beta}) \rightarrow U_{1}(\lambda \boldsymbol{s})$, erhalten wir für die komplexe Amplitude des in Richtung $\boldsymbol{\alpha}$ gebeugten Feldes

$$
U(\boldsymbol{\alpha}) \propto \mathcal{F}\left[U_{0}(\boldsymbol{\beta})\right]
$$

\subsection{Zur Bildentstehung}

Wir sind nun mit Hilfe der eingeführten Größen der beiden obigen Abschnitte in der Lage, der Frage nach der inkohärenten Bildentstehung nachzugehen. Jedes beliebig komplizierte optische System kann im linearen Fall als 'black box' angesehen werden, die eine bestimmte Wirkung auf die einlaufende Lichtwelle ausübt, die wiederum vollständig durch die Impulsantwort $h$ charakterisiert ist. Als solches können wir ein optisches, abbildendes System dadurch definieren, dass es Licht in endlicher Entfernung fokussiert und ein Bild erzeugt, wie in Abbildung 2.2 angedeutet. Dort bezeichnet $U_{0}(\boldsymbol{\beta})$ die Amplitude des Lichtwellenfeldes des Objektes in der Objektebene. Weiterhin ist $U(\boldsymbol{\alpha})$ die Feldamplitude in der Bildebene und $P(\mu)$ die Pupillenfunktion des abbildenden Systems und die Größen $\boldsymbol{\alpha}, \boldsymbol{\beta}$ sind die jeweiligen allgemeinen zweidimensionalen Winkelkoordinaten, $\boldsymbol{\mu}$ die räumliche Koordinate. Da die Austrittspupille und die Eintrittspupille Bilder voneinander sind, können alle Beugungseffekte mit einer von beiden assoziiert werden. 
Damit sind alle Einzelheiten des Systems in der Pupille spezifiziert. Nimmt man räumliche Isoplanatizität an und betrachtet eine mochochromatische Quelle, so kann man nach Gleichung 2.5 das resultierende Feld in der Bildebene schreiben als

$$
U(\boldsymbol{\alpha})=U_{0} * h=\int U_{0}(\boldsymbol{\beta}) h(\boldsymbol{\alpha}-\boldsymbol{\beta}) \mathrm{d} \boldsymbol{\beta}
$$

mit der Impulsantwort $h(\boldsymbol{\alpha}-\boldsymbol{\beta})$ des Systems. Da $h$ die Antwort des Systems auf eine Deltafunktion-Anregung darstellt, kann sie direkt erhalten werden, wenn wir für das Feld $U_{0}$ das einer Punktlichtquelle zulassen. Wir benutzen dabei die Fernfeld-Approximierung, da das System ein fokussiertes Bild liefert. Damit wird klar, dass die Impulsantwort proportional zur Fouriertransformierten der Pupillentransmissionsfunktion $P$ wird:

$$
h(\boldsymbol{\alpha}-\boldsymbol{\beta}) \propto \mathcal{F}[P(\boldsymbol{\mu})] .
$$

Bisher haben wir die Kohärenzeigenschaften des Lichtwellenfeldes vernachlässigt. Für quasi-monochromatische Quellen geschieht dies durch Hinzufügen einer zeitlichen Abhängigkeit, so dass $U_{0}(\boldsymbol{\beta}) \rightarrow U_{0}(\boldsymbol{\beta}, t)$ wird. Allerdings registrieren optische Detektoren für gewöhnlich Intensitäten, also das Betragsquadrat der Feldamplitude, und die Integrationszeiten sind um Größenordnungen länger als eine einzelne optische Schwingungsperiode. Deshalb muss die zeitliche Mittelung berücksichtigt werden, um die relevanten experimentellen Größen zu erhalten. Man muss lediglich darauf achten, die Betragsbildung erst an der Detektorebene auszuführen! Die Intensität $I(\boldsymbol{\alpha})$ in der Bildebene kann dann geschrieben werden als

$$
\begin{aligned}
I(\boldsymbol{\alpha}) & =\left\langle U(\boldsymbol{\alpha}, t) U^{*}(\boldsymbol{\alpha}, t)\right\rangle \\
& =\iint\left\langle U_{0}(\boldsymbol{\beta}, t) U_{0}^{*}\left(\boldsymbol{\beta}^{\prime}, t\right)\right\rangle h(\boldsymbol{\alpha}-\boldsymbol{\beta}) h^{*}\left(\boldsymbol{\alpha}-\boldsymbol{\beta}^{\prime}\right) \mathrm{d} \boldsymbol{\beta} \mathrm{d} \boldsymbol{\beta}^{\prime} .
\end{aligned}
$$

Hier muss nun zwischen dem kohärenten und inkohärenten Fall unterschieden werden. Für stellare Beobachtungsobjekte mit thermischem Emissionsspektrum kann sicherlich davon ausgegangen werden, dass die Quelle keinerlei räumliche Kohärenz aufweist. Somit wird im Integral der Gleichung 2.19 der Term in den spitzen Klammern nur für $\beta=\beta^{\prime}$ einen Beitrag liefern

$$
\left\langle U_{0}(\beta, t) U_{0}^{*}\left(\beta^{\prime}, t\right)\right\rangle=I_{0}(\beta) \delta\left(\beta-\beta^{\prime}\right) .
$$

Setzen wir diesen Ausdruck mit der Quellintensität $I_{0}(\beta)$ in 2.19 ein, erhalten wir die inkohärente Abbildungsgleichung

$$
\left.I(\boldsymbol{\alpha})=\int I_{0} \boldsymbol{\beta}\right)|h(\boldsymbol{\alpha}-\boldsymbol{\beta})|^{2} \mathrm{~d} \boldsymbol{\beta}=I_{0} *|h|^{2} .
$$

Die Funktion $|h|^{2}$ ist die Energieverbreiterungsfunktion ESF (Energy Spread Function) und wird in ihrer normierten Form als Punktverbreiterungsfunktion PSF (Point Spread Function) bezeichnet und ist von zentraler Bedeutung: sie beschreibt eine durch das optische System abgebildete Punktlichtquelle und stellt als Impulsantwort ein fundamentales Maß für die Bildqualität dar. Fassen wir die Beschreibung in der Bildebene mit Hilfe der dimensionslosen Koordinaten aus Gleichung 2.14 zusammen:

$$
\begin{aligned}
I(\boldsymbol{\alpha}) & =I_{0}(\boldsymbol{\beta}) * E S F(\boldsymbol{\alpha}) \\
E S F(\boldsymbol{\alpha}) & =|\mathcal{F}[P(\lambda \boldsymbol{s})]|^{2}
\end{aligned}
$$


Die PSF ist außerdem die Fouriertransformierte der optischen Übertragungsfunktion OTF (Optical Transfer Function). Die OTF ist die am Ursprung auf Eins normierte Energieübertragungsfunktion ETF (Energy Transfer Function), d. h. die PSF ist entsprechend auf den gesamten Energieinhalt normiert, also auf ihr Integral über die gesamte Bildebene. Es gilt also

$$
\begin{aligned}
O T F(\boldsymbol{s}) & =\frac{\operatorname{ETF}(\boldsymbol{s})}{\operatorname{ETF}(0)}=\mathcal{F}[\operatorname{PSF}(\boldsymbol{\alpha})] \\
\mathcal{F}[I](\lambda \boldsymbol{s}) & \propto \mathcal{F}\left[I_{0}(z \boldsymbol{\alpha}) * \operatorname{PSF}(\boldsymbol{\alpha})\right]=\mathcal{F}\left[I_{0}\right](\lambda \boldsymbol{s}) \cdot \operatorname{OTF}(\boldsymbol{s}) .
\end{aligned}
$$

Während die PSF Aussagen zur Energieverteilung im Bild einer Punktlichtquelle macht, beschreibt die OTF die Übertragung der räumlichen Frequenzen des Bildes zusammen mit der Phaseninformation. Mit Hilfe des Wiener-Khinchine-Theorems erhalten wir aus G1. 2.23 auch noch die Beziehung

$$
O T F(\boldsymbol{s})=\frac{P \star P}{E T F(0)}=\frac{1}{E T F(0)} \int P\left(\boldsymbol{x}_{\mathbf{1}}\right) P^{*}\left(\boldsymbol{x}_{\mathbf{1}}^{\prime}\right) \mathrm{d} \boldsymbol{x}_{\mathbf{1}},
$$

die es erlaubt, die OTF als Autokorrelationsfunktion ' $\star$ ' der Pupillenfunktion $P$ zu schreiben.

\subsection{Aberrationsfreies optisches System}

Die Leistung eines jeden abbildenden Systems kann zweckmäßigerweise aus einem Vergleich der tatsächlichen PSF mit einer idealen Referenz abgeleitet werden, wozu allerdings die Pupillenfunktion des Systems bekannt sein muss. Im idealen Fall der perfekten Abbildung wird das Ergebnis nur von der geometrischen Form der Blendenöffnung abhängen. Im Falle einer kreisrunden Teleskopöffnung mit Durchmesser $D$ ohne Zentralabschattung ist die Pupillenfunktion $P$ definiert durch

$$
P(\boldsymbol{r})=\operatorname{zirk}(\boldsymbol{r}), \quad \text { wobei } \quad \operatorname{zirk}(\boldsymbol{r})= \begin{cases}1 & \text { für } \quad r \leq D / 2 \\ 0 & \text { sonst }\end{cases}
$$

Um die zugehörige PSF zu erhalten, bildet man zunächst die Fouriertransformierte, die für zweidimensionale, kreissymmetrische Funktionen eine Hankeltransformation null-ter Ordnung $\mathcal{H}$ darstellt:

$$
\mathcal{H}[f(r)](\kappa)=2 \pi \int_{0}^{\infty} r f(r) J_{0}(2 \pi \kappa r) \mathrm{d} r
$$

$J_{0}$ bezeichnet die Besselfunktion null-ter Ordnung und $\kappa$ ist definiert durch $\kappa^{2}=\kappa_{x}^{2}+\kappa_{y}^{2}$ und bedeutet die radiale Raumfrequenzkoordinate in den ursprünglichen Variablen $\kappa=$ $\boldsymbol{x}_{1} / \lambda z=|\boldsymbol{\alpha}| / \lambda$. Für eine Kreisscheibe vom Durchmesser D ist die Impulsantwort in reduzierten Variablen (Gl.2.14) gegeben durch die Hankeltransformation

$$
\mathcal{H}\left[\operatorname{zirk}\left(\frac{\lambda s}{D}\right)\right]=\frac{(D / 2) J_{1}(\pi D \alpha / \lambda)}{\alpha / \lambda}=\frac{\pi D^{2}}{4}\left[\frac{2 J_{1}(\pi D \alpha / \lambda)}{\pi D \alpha / \lambda}\right],
$$


mit der Notation $\alpha=|\boldsymbol{\alpha}|$ und $s=|\boldsymbol{s}|$. Zur PSF gelangen wir nun endlich, indem wir diesen Ausdruck quadrieren und normieren:

$$
P S F_{t e l}(\alpha)=\frac{1}{\pi}\left[\frac{J_{1}(\pi D \alpha / \lambda)}{\pi D \alpha / \lambda}\right]^{2} .
$$

Ihre funktionale Form wird üblicherweise als Airy-Figur bezeichnet und stellt für ein beugungsbegrenztes Teleskop das Bild der Intensitätsverteilung einer punktförmigen Lichtquelle dar, also insbesondere die von Sternen. Analog zur Normierung der PSF auf ihr Integral ist die zugehörige optische Übertragungsfunktion

$$
O T F_{t e l}(s)=P \star P=\frac{2}{\pi}\left\{\arccos (|s|)-|s| \sqrt{1-|s|^{2}}\right\} \quad(|s| \leq 1)
$$

auf ihren Wert am Ursprung normiert.

Die Abbildung ausgedehnter Objekte kann nun als Überlagerung der Airy-Figuren jedes Objektpunktes auf dem Detektor gesehen werden. Die kleinsten auflösbaren Details im Bild sind dann von der Ordnung des Durchmessers des ersten Nullstellenringes des Airy-Musters (Airy-Scheibchen)

$$
D_{A}=\frac{2.44 \lambda}{D}[\mathrm{rad}]
$$

Da die Pupille P notwendigerweise räumlich begrenzt ist, wirkt die Fouriertransformation als Tiefpass-Filter für die Raumfrequenzen des Objektbildes. Da die hohen Frequenzen die Feinstruktur des Objekts kodieren, wird das Bild dadurch unscharf. Wie aus der Skalierungseigenschaft der Fouriertransformation folgt (siehe G1.2.28), muss letztendlich die Eintrittspupille vergrößert und damit die Breite der PSF verringert werden, wenn höhere räumliche Auflösung erzielt werden soll.

Im Frequenzraum betrachtet, bewirkt die räumliche Begrenzung der Apertur des Teleskops auch eine Limitierung der Bandbreite der OTF. Das Signal fällt dann bei einer maximalen Frequenz auf Null ab, die inkohärente cut-off Frequenz $f_{c}$ genannt wird. Für unser Beispiel einer kreisförmigen Eintrittsöffnung wird sie zu

$$
f_{c}=\frac{D}{\lambda} \quad\left[\mathrm{rad}^{-1}\right]
$$

Für die Bemessung der Bildqualität ist die OTF eine gleichwertige Alternative zur PSF, wobei Formel 2.33 für jede Pupille mit (größtem) Durchmesser D gilt, egal wie ihr Inneres aussieht, insbesondere gilt sie auch für den Fall einer Zentralabschattung durch einen Primärspiegel.

Nach der Beleuchtung des Idealfalles einer optischen Abbildung wenden wir uns nun der Realität zu und müssen uns daher mit Aberrationen befassen. Uns interessieren dabei jedoch nicht so sehr solche, die durch Instrumentierung verursacht sind, als die von atmosphärischer Turbulenz Herrührenden, da es ihre zufälligen Fluktuationen sind, die eine kontinuierliche Beobachtung und adaptive Korrektur erfordern, wie sie AO-Systeme bieten. Statische und langsam veränderliche (quasistatische) Aberrationen erfordern sehr viel weniger technischen Aufwand und können oft mit permanenten Lösungen behoben werden. 


\section{Turbulenz der Erdatmosphäre}

\subsection{Allgemeines}

Hier soll ein Überblick über die wichtigsten Eigenschaften der Erdatmosphäre gegeben werden, insofern sie die einlaufende Strahlung von astrophysikalischen Quellen beeinflusst. Die Diskussion wird sich auf die Effekte der Turbulenz konzentrieren. Statistische Momente des stochastischen Prozesses, als den wir die Turbulenz sehen, werden mit Bezug auf das Kolmogorov'sche Modell beschrieben. Die Phasenstrukturfunktion und der Fried-Parameter $r_{0}$ werden eingeführt und analytische Ausdrücke für die Degradationen der optischen Transferfunktion durch die Atmosphäre, für die resultierende Strehl'sche Definitionshelligkeit, sowie für den Anisoplanatismus abgeleitet.

Die Erdatmosphäre dehnt sich bis zu einer Höhe von etwa $300 \mathrm{~km}$ aus. Ihr Druck nimmt dabei gleichmäßig von etwa 1000 mbar auf Meereshöhe gemäß einem exponentiellen Gesetz ab, mit einem anfänglichen Abfall von 12.1 mbar pro $100 \mathrm{~m}$. Dagegen misst man ein ganz anderes Höhenprofil der für die Abbildungsqualität wichtigen Turbulenzverteilung, denn man findet vornehmlich dünne Schichten von Ausdehnungen bis herunter zu $100 \mathrm{~m}$. Es sind hauptsächlich Grenzschichten von Luftmassen verschiedener Bewegungsrichtung und Geschwindigkeit (Näheres zum Turbulenzprofil in Abschnitt 3.2.6). Aus globaler Sicht ergeben sich deswegen die optimalen Standorte für astronomische Beobachtungen, nämlich auf möglichst isolierten, hohen Berggipfeln im Ozean oder an dem Wind zugewandten Ozeanküsten. Dort werden Temperaturunterschiede durch die Wassermassen gedämpft und die Windverhältnisse werden nicht durch geographische Hindernisse gestört. Bezüglich der allgemeinen Struktur der globalen Luftzirkulation bieten niedrige Breiten bis $35^{\circ}$ stabilere und gleichmäßigere Windverhältnisse.

\subsection{Mechanik der Turbulenz}

\subsubsection{Ursachen}

Die eigentliche Energiequelle für Luftbewegungen in der Erdatmosphäre ist die Aufwärmung durch Sonneneinstrahlung, indem sich deswegen tagsüber lokale Konvektionszellen bilden. Nachts bildet das Vermischen von Luftmassen unterschiedlicher Temperatur durch Wind die Hauptenergiequelle. 


\subsubsection{Strömungsmechanische Größen}

Die Reynoldszahl ist definiert als $R e=v_{0} L_{0} / \nu_{0}$, mit der charakteristischen Geschwindigkeit $v_{0}$, der charakteristischen Länge $L_{0}$ und der kinematischen Viskosität $\nu_{0}$ der Strömung. Nimmt man für Luft $\left(\nu_{0}=15 \cdot 10^{-6} \mathrm{~m}^{2} \mathrm{~s}^{-1}\right)$ mäßig große atmosphärische Störungen mit $L_{0}=15 \mathrm{~m}$ bei einer Geschwindigkeit $v_{0}=1 \mathrm{~m} / \mathrm{s}$ an, erhält man eine Reynoldszahl $R e=1 \cdot 10^{6}$, die den kritischen Wert $R e \approx 100$ bis 1000 um Größenordnungen übertrifft.

\subsubsection{Statistische Beschreibung}

Die Mittelwerte vieler meteorologischer Variablen, wie Temperatur, Druck oder Luftfeuchtigkeit, verändern sich kontinuierlich innerhalb von Minuten bis Stunden. Der Unterschied der Mittelwerte kann dabei beträchtlich werden und bei der Analyse der Variablen von langsamen Fluktuationen nicht unterschieden werden. Wie in (Tatarskij 1961) diskutiert wird, kann dieses Problem, im Fall einer nichtstationären Zufallsfunktion $f(t)$ mit zeitlich veränderlichem Mittelwert, dadurch umgangen werden, dass die Differenzfunktion $F_{t}(\tau)=f(t+\tau)-f(t)$ verwendet wird. Für nicht zu große $\tau$ kann $F_{t}(\tau)$ dann als stationär angenommen werden ${ }^{1}$. Damit kann nun eine Strukturfunktion

$$
D_{f}(\tau)=\left\langle\left[F_{t}(\tau)\right]^{2}\right\rangle=\left\langle[f(t+\tau)-f(t)]^{2}\right\rangle
$$

definiert werden, wie sie von Kolmogorov (1941) in die Turbulenztheorie zur Beschreibung räumlicher Charakteristika des Mediums eingeführt wurden. Die Strukturfunktion ist ein Maß für die Intensität der Fluktuationen in $f(t)$ über Perioden etwa der Länge $\tau$. Für eine als stationär angenommene Funktion $f(t)$ ergibt sich folgender Zusammenhang mit der als $B_{f}(\tau)=\left\langle f(t+\tau) f^{*}(t)\right\rangle$ definierten Korrelationsfunktion:

$$
D_{f}(\tau)=2\left[B_{f}(0)-B_{f}(\tau)\right]
$$

$B_{f}(0)$ bezeichnet das mittlere Quadrat der Funktion. Da bei den meisten Funktionen die Korrelation bei großen Abständen $\tau$ verschwindet, erhalten wir bei Mittelwert Null also die doppelte Varianz der Fluktuationen, wie wir es für zwei unabhängige Größen auch erwarten, als Grenzwert, $D_{f}(\infty)=2 B_{f}(0)$. Dies gilt insbesondere bei atmosphärischer Turbulenz.

\subsubsection{Kolomogorov'sches Modell}

Kolmogorov (1941) untersuchte die mechanische Struktur der Turbulenz (Geschwindigkeit von Bewegungen in dem fluiden Medium), um ein Modell der zugrundeliegenden physikalischen Prozesse zu entwickeln. Dies war nötig, um solch ein komplexes, stochastisches Phänomen wie die atmosphärische Turbulenz zu analysieren. Das Modell nimmt an, dass dem Fluid Energie in Form von großskaligen Störungen ('outer scale', $L_{0}$ ) zugefügt wird, die dann in immer kleinere Strukturen zerfallen, sodass die kinetische Energie

\footnotetext{
${ }^{1}$ Es sei angemerkt, dass die Argumente $t$ und $\tau$ insbesondere auch als räumliche Variablen mit ähnlichen statistischen Eigenschaften gesehen werden können.
} 
in einer Kaskade weitergegeben wird, bis die Reynoldszahl den kritischen Wert unterschreitet und die Energie durch Reibung in Wärme dissipiert werden kann ('inner scale', $l_{0}$ ). Dieses Verhalten hat universellen Charakter und tritt insbesondere bei turbulenten Strömungen auf, also immer, wenn die Reynoldszahl über einen kritischen Wert hinauswächst. Wie oben gesehen verhalten sich Luftströmungen in der Atmosphäre praktisch immer turbulent. Die Größe von $L_{0}$ wurde in der Literatur intensiv diskutiert und bewegt sich in der Größenordnung von einigen $10 \mathrm{~m}$ bis $1 \mathrm{~km}$. Sicher ist, dass sie sehr variabel und höhenabhängig ist (Coulman et al. 1988). In der Sonnenbeobachtung hat man es zudem mit kleineren Skalen zu tun, da die Sonneneinstrahlung zu größerer Turbulenz führt als nachts. Schätzungen variieren im Bereich von $1 \mathrm{~m}$ bis $100 \mathrm{~m}$ ! Der Teleskopdurchmesser kann also bei der Sonnenbeobachtung in guter Näherung als klein gegenüber der oberen Turbulenzskala gelten. Die andere Grenze, $l_{0}$, geht bei Kenntnis der Reynoldszahl gemäß der Relation

$$
l_{0}=\frac{L_{0}}{(R e)^{3 / 4}}
$$

aus $L_{0}$ hervor, wird also größer, wenn die Geschwindigkeit der Strömung wächst. Nach Roddier (1981) bewegt sich $l_{0}$ von etwa $1 \mathrm{~mm}$ am Boden bis etwa $1 \mathrm{~cm}$ nahe der Tropopause. Die Skalen $l_{0}$ und $L_{0}$ begrenzen den sogenannten 'inertialen Bereich'.

Damit sich nun aber auch ein stabiles dynamisches Gleichgewicht ausbilden kann, muss die Dissipationsrate gleich der Rate der zugeführten Energie sein. Diese Bedingung impliziert nun, dass die Geschwindigkeitsfluktuationen $V$ auf jeder Größenskala innerhalb des inertialen Bereichs nur von der Skalengröße $l$ und der Energietransportrate $\epsilon$ abhängen kann. So wird eine Dimensionsbetrachtung möglich, die zu der Beziehung

$$
V \propto \epsilon^{1 / 3} l^{1 / 3}
$$

führt. Man sieht, dass die zur Turbulenzzellengröße $l$ gehörenden Energiefluktuationen proportional zu $l^{2 / 3}$ sind. Außerdem kann man damit das Leistungsspektrum der Turbulenz ableiten: Mit der räumlichen Wellenzahl $\kappa=2 \pi / l$ schreiben wir die Leistungsdichte der Fluktuationen als $\Phi(\kappa)$. Im Inkrement $d \kappa$ bedeutet die Fläche unter ihrem Graphen die zugehörige Energie, die wiederum proportional zu $V^{2}$ ist. Aus Gleichung 3.4 und der Definition von $\kappa$ ergibt sich aber auch die Proportionalität $\mathrm{zu} \kappa^{-2 / 3}$. Folglich ergibt sich für $\Phi$

$$
\Phi(\kappa) d \kappa \propto \kappa^{-2 / 3} \Rightarrow \Phi(\kappa) \propto \kappa^{-5 / 3} .
$$

Diese Beziehung definiert das Kolmogorov'sche Spektrum in einer Dimension. Für die Analyse der Wellenfrontpropagation durch Turbulenz benötigt man jedoch das dreidimensionale Spektrum $\Phi(\boldsymbol{\kappa})=\Phi\left(\kappa_{x}, \kappa_{y}, \kappa_{z}\right)$. Eindimensionale Spektren werden aus dreidimensionalen Spektren durch Integration über alle Richtungen gewonnen. Im isotropen Fall haben wir also, wie Tatarskij (1961) gezeigt hat,

$$
\Phi(\kappa)=4 \pi \kappa^{2} \Phi(\boldsymbol{\kappa}) \quad \Rightarrow \quad \Phi(\boldsymbol{\kappa}) \propto \kappa^{-11 / 3} .
$$

Obwohl ein sehr einfaches Modell, beschreibt Kolmogorov's 'Zwei Drittel Gesetz' doch die meisten beobachteten Phänomene in guter Näherung und wurde deshalb weithin akzeptiert. Beschränkungen für das Potenzgesetz ergeben sich jedoch aus seiner Gültigkeit ausschließlich innerhalb des inertialen Bereichs und in der Annahme eines inkompressiblen Mediums mit isotroper Turbulenz. Für die Adaptive Optik und speziell für die 
Simulation realistischer Phasenschirme von Wellenfronten ist die Kenntnis von $L_{0}$ nun wichtig, da sie die Größe der Gesamt-Tip-Tilt-Komponente der atmosphärischen Turbulenz bestimmt. Wird die Bedingung $L_{0} \gg D$ für den Teleskopdurchmesser nicht mehr erfüllt, dann überschätzt das Modell den tatsächlichen Tip-Tilt-Anteil und man sollte etwa das von Kármán-Spektrum 3.13 verwenden. Hinzu kommt, dass die 'outer scale' nach Beckers (1993) nicht unbedingt eine wohlbestimmte, einzelne Größe ist. Energie kann der atmosphärischen Turbulenz auf vielen verschiedenen Skalen zugeführt werden, sei es durch Sonneneinstrahlung, Wind an Grenzschichten oder auch die lokale Umgebung des Teleskops, sodass zu jedem Zeitpunkt auch das effektive $L_{0}$ von der jeweils dominierenden Quelle abhängt. Im Übrigen wurden gerade in höheren atmosphärischen Schichten auch Abweichungen von der Isotropie der Turbulenz gemessen, die den Exponenten im Leistungsdichtespektrum 3.6 senken (Beyer et al. 2003).

\subsubsection{Turbulente Brechungsindexvariationen}

Wäre die Atmosphäre ein Medium in thermischem Gleichgewicht und wiese keine Luftfeuchtigkeitsschwankungen auf, so produzierte die mechanische Turbulenz keinerlei optische Effekte. Diese werden durch Variationen des Brechungsindex hervorgerufen. Für seine Fluktuationen spielt der Wassergehalt der Luft bei haupsächlich vertikalen Lichtwegen nur eine vernachlässigbare Rolle, sodass es die durch Turbulenz vermischten Luftschichten unterschiedlicher Temperatur sind, die über lokale Dichtefluktuationen die abbildenden Eigenschaften der Atmosphäre bestimmen. Ausgehend von Gleichung 3.4 kann man für ein lokal homogenes und isotropes Medium die Strukturfunktion zwischen zwei Komponenten der Geschwindigkeiten an zwei um $r$ auseinanderliegenden Punkten auf der x-Achse als

$$
D_{v}(r)=|v(x)-v(x+r)|^{2}=C_{v}^{2} r^{2 / 3}
$$

definieren, wobei der Koeffizient $C_{v}^{2}$ von der Energie des Prozesses abhängt. Die mechanische Turbulenz ist nun die treibende Ursache für die Vermischung der Luft aus verschiedenen Höhen, deswegen liegt nahe, dass auch die Temperaturfluktuationen durch eine formal gleiche Strukturfunktion beschrieben werden kann. Tatsächlich gilt (siehe Obukhov 1949)

$$
D_{T}(r)=|T(x)-T(x+r)|^{2}=C_{T}^{2} r^{2 / 3}
$$

mit dem Strukturparameter $C_{T}^{2}$ für Temperaturänderungen. Die Brechkraft $N$ der Luft hängt bei vertikalen Lichtwegen nach

$$
N=(n-1) 10^{6}=77.6 P / T
$$

von Temperatur $T$ und Druck $P$ ab, wenn als Einheiten Kelvin bzw. millibar benutzt werden. Druckdifferenzen werden schnell, mit Schallgeschwindigkeit, ausgeglichen, sodass Brechungsindexfluktuationen $\delta N$ als

$$
\delta N=-77.6\left(\frac{P}{T^{2}}\right) \delta T
$$

dargestellt werden dürfen. Für die Strukturfunktion des Brechungsindex folgt damit

$$
C_{N}=\frac{\delta N}{\delta T} C_{T} \quad \Rightarrow \quad D_{N}(r)=C_{N}^{2} r^{2 / 3}
$$



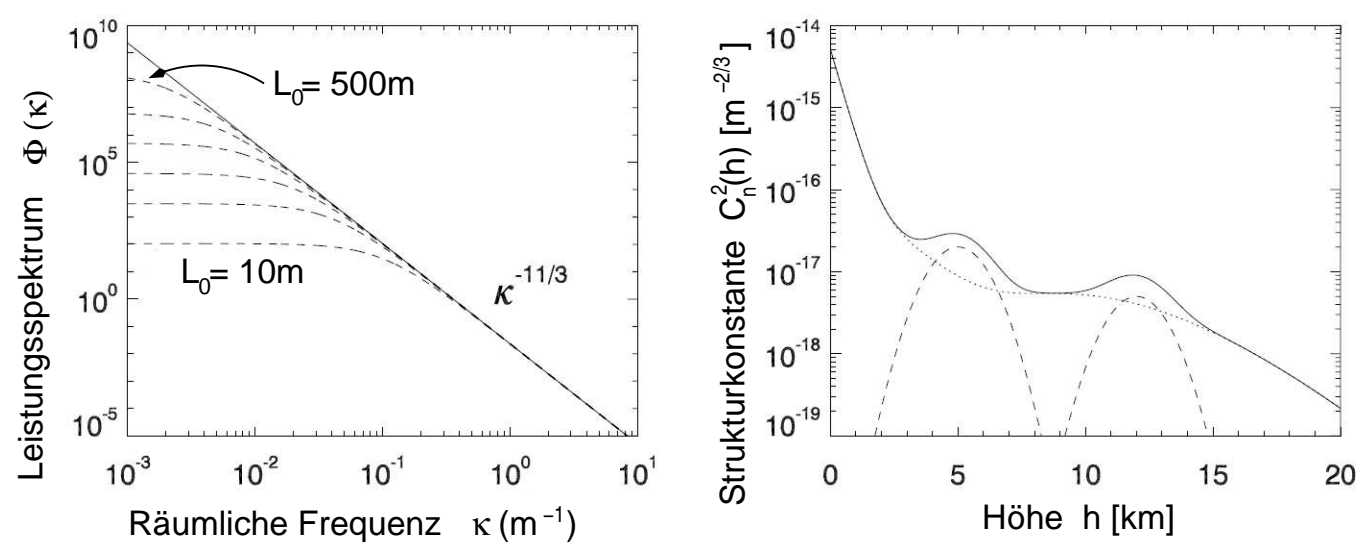

Abbildung 3.1: Links: Theoretische Leistungsspektren nach Komogorov (durchgezogene Linie) und nach von Kármán (gestrichelte Linien) jeweils bei einem Wert aus \{10, 25, 50, $100,200,500\}$ in Metern für die 'outer scale' $L_{0}$. Rechts: Beispiel für ein analytisches $C_{n}^{2}$ Modellprofil nach Hufnagel-Valley (durchgezogene Linie). Der punktierte Verlauf zeigt den Beitrag der oberflächennahen Schicht, der planetaren Grenzschicht und der Tropopause. Die unterbrochenen Linien illustrieren, wie der Beitrag von zwei unterschiedlichen Wind-Scherschichten, hier bei 5 und $12 \mathrm{~km}$, modelliert wird.

in formaler Übereinstimmung mit 3.8 und 3.7. Mit Hilfe der Strukturparameter kann auch das Leistungsdichtespektrum des Brechungsindex ausgedrückt werden, wie in Tatarskij (1961) gezeigt wurde:

$$
\Phi_{N}(\kappa)=0.033 C_{N}^{2} \kappa^{-11 / 3} .
$$

Wie oben ausgeführt, ist die Form des Spektrums außerhalb der Werte $L_{0}$ und $l_{0}$ unsicher. Oft ist es nämlich notwendig, über alle Wellenzahlen zu integrieren, um Voraussagen über die optischen Effekte der Turbulenz treffen zu können. Der nicht integrierbare Pol bei $\kappa \rightarrow 0$ führt im Kolmogorov-Spektrum 3.12 auf unphysikalische Resultate, wie z. B. eine unendliche Varianz der Brechungsindexfluktuationen. Um dieses Problem zu überwinden, hat von Kármán folgendes Spektrum vorgeschlagen (siehe Roggemann \& Welsh 1996):

$$
\Phi_{n}(\kappa)=\frac{0.033 C_{N}^{2}}{\left(\kappa^{2}+\kappa_{0}^{2}\right)^{11 / 6}} \exp \left(-\frac{\kappa^{2}}{\kappa_{m}^{2}}\right)
$$

mit $\kappa_{m}=5.92 / l_{0}$ und $\kappa_{0}=2 \pi / L_{0}$, die dafür sorgen, dass das Spektrum bei hohen Frequenzen exponentiell gedämpft wird und bei niedrigen Frequenzen asymptotisch konstant verläuft, sodass die Varianz endlich bleibt. Neben dem Kolomogorov'schen Fall sind einige nach von Kármán berechneten Spektren mit verschiedenen Werten für die obere Grenzskala der Turbulenz in Abbildung 3.1 dargestellt.

\subsection{6 $C_{n}^{2}$-Höhenprofil}

Das Turbulenzprofil der Atmosphäre hat fundamentalen Einfluss auf das Design eines passenden AO-Systems. Wie eingangs erwähnt, weist die Turbulenzstärkenverteilung eine mehr oder weniger ausgeprägte 'diskrete' Struktur auf. In diesen dünnen Schichten von 
100 bis $200 \mathrm{~m}$ Dicke steigt der Brechungsindexstrukturkoeffizient $C_{n}^{2}$ um gut eine Größenordnung. Der Bereich, in dem die Schichten auftreten ('planetary boundary layer'), reicht von etwa $1 \mathrm{~km}$ bis etwa $9 \mathrm{~km}$ und $C_{n}^{2}$ nimmt dabei bis auf $10^{-17} \mathrm{~m}^{-2 / 3} \mathrm{ab}$. In der Nachtastronomie tragen (bei exzellenten Beobachtungsorten) vor allem höher gelegene Schichten zur gesamten Turbulenzstärke bei, insbesondere die Tropopause in etwa 10 km Höhe als Scherschicht ausgleichender Winde. Tagsüber bilden sich jedoch infolge der Erwärmung durch Sonneneinstrahlung in der bodennahen (bis $1 \mathrm{~km}$ ) Luftschicht die stärksten Turbulenzen. Oberhalb der Troposphäre nimmt $C_{n}^{2}$ schnell ab, die optischen Effekte der Turbulenz verschwinden in einer Höhe von etwa $25 \mathrm{~km}$ über Grund und oberhalb $30 \mathrm{~km}$ findet man bei einem Druck von unter 10 mbar kaum mehr Beiträge zur gesamten atmosphärischen Turbulenz. Ein Beispiel für ein $C_{n}^{2}$-Profil, das innerhalb eines analytischen Hufnagel-Valley-Modells (Valley 1980) gerechnet ist, ist in Abbildung 3.1 abgebildet.

Natürlich gibt es noch lokale Quellen von Turbulenz, die nicht im $C_{n}^{2}$-profil auftauchen, etwa innerhalb der Teleskopstruktur oder der Kuppel. Die damit verbundenen Temperaturunterschiede können aber meist, ob aktiv oder passiv, ausreichend klein gehalten werden.

\subsection{Momente und Kohärenz}

Viele fundamentale Eigenschaften von stochastischen Prozessen können durch ihre verschiedenen Momente quantifiziert werden. Zwei davon sind beim Studium der atmosphärischen Turbulenz von großer Nützlichkeit, nämlich die $m$-ten Momente der Turbulenzstärke $\mu_{m}$ und der Windgeschwindigkeit $v_{m}$, definiert durch die Ausdrücke

$$
\begin{aligned}
& \mu_{m}=\sec ^{m+1}(\zeta) \int_{0}^{\infty} C_{n}^{2}(h) h^{m} \mathrm{~d} h \quad \text { und } \\
& v_{m}=\sec ^{m+1}(\zeta) \int_{0}^{\infty} C_{n}^{2}(h) v^{m}(h) \mathrm{d} h,
\end{aligned}
$$

mit dem Windgeschwindigkeitsprofil $v(h)$, einem Faktor $\sec (\zeta)$, der den längeren Lichtweg durch die Atmosphäre bei schrägem Lichteinfall unter dem Zenitwinkel $\zeta$ berücksichtigt. Für verschiedene Geltungsbereiche können im Kolmogorov-Modell folgende quantitative Maße für die Kohärenz der Turbulenz angegeben werden:

$$
\begin{aligned}
r_{0}^{-5 / 3} & =0.423 k^{2} \mu_{0}, \\
\theta_{0}^{-5 / 3} & =2.91 k^{2} \mu_{5 / 3}, \\
\tau_{0}^{-5 / 3} & =0.102 k^{2} v_{5 / 3},
\end{aligned}
$$

wobei $k=2 \pi / \lambda$ die Wellenzahl und $\lambda$ die Wellenlänge des Lichtes sind. Diese drei Parameter charakterisieren in dieser Reihenfolge die räumlichen, winkelbezogenen und zeitlichen Kohärenzeigenschaften Kolmogorov'scher Turbulenz. Die Größen sind feste Bestandteile des Grundwortschatzes auf dem Gebiet der Adaptiven Optik geworden und wurden alle von Fried (1965, 1966a) eingeführt, wobei ihm zu Ehren die räumliche Kohärenzlänge $r_{0}$ gewöhnlich als Fried-Parameter genannt wird. Er hat eine Reihe von nützlichen Interpretationen, entspricht aber zahlenmäßig dem Durchmesser eines Lichtbündels, 
das nach Durchlaufen Kolmogorov'scher Turbulenz eine Phasenvarianz $\sigma_{\phi}^{2} \simeq 1 \operatorname{rad}^{2}$ aufweist. Dies bedeutet wiederum einen Kohärenzgrad der Energie von 0.37 (Strehl-Zahl, siehe 3.30), der um den Faktor zwei niedriger liegt, als üblicherweise als 'fast beugungsbegrenzt' gilt.

Der isoplanatische Winkel $\theta_{0}$ (Fried 1982) beschreibt die Feldwinkelabhängikeit der Turbulenz und entspricht demjenigen Winkel, bei dem der Versatz zweier Lichtbündel in der Höhe der Turbulenzschicht zu einer Phasenvarianz von $1 \mathrm{rad}^{2}$ führt. Da meistens nicht von nur einer einzelne Turbulenzschicht ausgegangen werden kann, definiert man gewöhnlich eine mittlere Turbulenzhöhe $\bar{h}=\left(\mu_{5 / 3} / \mu_{0}\right)^{3 / 5}$, über die der isoplanatische Winkel zum Fried-Parameter $r_{0}$ durch

$$
\theta_{0}=0.314 \cos (\zeta) \frac{r_{0}}{\bar{h}}
$$

in Beziehung gesetzt werden kann. Die Kohärenzzeit $\tau_{0}$ atmosphärischer Turbulenz hängt nach Fried (1990) mit der Greenwood-Frequenz $f_{G}($ Greenwood 1977$)$ wie $\tau_{0}=0.143 / f_{G}$ zusammen. Ähnlich kann ein quadratischer Phasenfehler von $1 \mathrm{rad}^{2}$ zwischen zwei Ensembles von Komogorov'scher Turbulenz auftreten, wenn sie durch das Windprofil $v(h)$ für die zeitliche Dauer $\tau_{0}$ gegeneinander verschoben werden. Die Definition $f_{G}$ und $\tau_{0}$ beruhen auf der Taylorhypothese, unter der Turbulenz als eine Gruppe von unendlich dünnen horizontalen Schichten modelliert wird. Ist die Belichtungsdauer einer Aufnahme kurz genug, kann die zeitliche Turbulenzentwicklung innerhalb der einzelnen Schichten als ,eingefroren“ angesehen werden, sodass sich die Schichten allein durch die unterschiedlichen Windgeschwindigkeiten und -richtungen gegeneinander verschieben. Auf Zeitskalen von der Ordnung $\tau_{0}$ oder kürzer, ist dies normalerweise eine gute Näherung und wird oft benützt. Die Werte von $r_{0}, \theta_{0}$ bzw. $\tau_{0}$ sind im sichtbaren Wellenlängenbereich von der Größenordnung einiger Zentimeter, Bogensekunden bzw. Millisekunden. Es sollte jedoch noch einmal darauf hingewiesen werden, dass die Definitionen 3.16 bis 3.18 aus strikt Kolmogorov'scher Turbulenz abgeleitet sind und nur dann gelten.

\subsection{Aberration durch Turbulenz}

Für die optischen Effekte der atmosphärischen Turbulenz ist die Phasenstrukturfunktion $D_{\phi}$ besser zu gebrauchen, als das Leistungsspektrum der Brechungsindexfluktuationen 3.12, da bei Rechnungen und Simulationen gerade die Polstelle bei $\kappa=0$ Schwierigkeiten macht. Nach Obukhov (1949) ist $D_{\phi}$ aus den Brechungsfluktuationen mit Hilfe des Fermat'schen Prinzip ableitbar und gegeben durch

$$
D_{\phi}=\left\langle\left|\phi\left(r_{0}\right)-\phi\left(r_{0}+r\right)\right|^{2}\right\rangle=2.91 k^{2} C_{n}^{2} r^{5 / 3} \delta h .
$$

Diese Gleichung gibt die durchschnittliche Phasendifferenz zwischen zwei parallelen Lichtstrahlen mit der Wellenzahl $k=2 \pi / \lambda$ an, wenn sie mit einem Abstand $r$ voneinander durch eine Turbulenzschicht der Dicke $\delta h$ und Strukturkonstanten $C_{n}^{2}$ treten. Da die Atmosphäre aber als kontinuierliches turbulentes Medium anzusehen ist, müssen die Phasendifferenzen über alle Höhen $h$ integriert werden, also

$$
D_{\phi}(r)=2.91 k^{2} \sec (z) \int_{0}^{\infty} C_{n}^{2}(h) r^{5 / 3} \mathrm{~d} h
$$


wobei mit $\sec (z)$ der längere Weg durch die Atmosphäre bei nicht-vertikalem Lichteinfall berücksichtigt wird. Die Gleichung impliziert die Einführung des Fried-Parameters $r_{0}$ (Fried (vgl. 1965) und Gl. 3.16)

$$
\left.r_{0}=\left(0.423 k^{2} \sec (z) \int_{0}^{\infty} C_{n}^{2}(h) \mathrm{d} h\right)\right)^{-3 / 5}
$$

mit welcher wir Gleichung 3.21 in die übersichtlichere Form

$$
D_{\phi}(r)=6.88\left(\frac{|\boldsymbol{r}|}{r_{0}}\right)^{5 / 3}
$$

umschreiben können. Der Fried-Parameter $r_{0}$ ist eine ungemein nützliche Größe für die Charakterisierung der atmosphärischen Turbulenz, da er zwei recht intuitive physikalische Interpretationen besitzt:

- $r_{0}$ entspricht dem Durchmesser derjenigen (kreisförmigen) Teleskopeintrittsöffnung, über die der $r m s$-Wert (root mean square) der Phasenaberrationen der einlaufenden Wellenfront etwa einem radian entspricht

- $r_{0}$ definiert den maximalen Durchmesser eines Teleskops, das bei gegebenen atmosphärischen Bedingungen noch beugungsbegrenzt abbildet, also seine höchste, theoretisch mögliche Winkelauflösung erreicht.

Bei einer Beobachtungswellenlänge von $500 \mathrm{~nm}$ bewegt sich $r_{0}$ gewöhnlich zwischen 5 und $15 \mathrm{~cm}$, also deutlich kleiner als typische Teleskopdurchmesser in der Astronomie, die Sonnenbeobachtung eingeschlossen! Daher kann nun die Auflösung ('Schärfe') der Aufnahmen bei unkorrigierten Langzeit-Belichtungen nicht durch eine Vergrößerung des Teleskops erreicht werden, nur die Empfindlichkeit erhöht sich aufgrund der erweiterten Lichtsammelfläche. Da die statistischen Eigenschaften der Phasenaberrationen nun bekannt sind, können wir den Effekt der Turbulenz auf die Abbildungsqualität untersuchen.

\subsubsection{Inkohärente Übertragungsfunktion}

Ein allgemeines Abbildungssystem, das ein im Unendlichen gelegenes Objekt fokussiert, wie es die meisten astronomischen Systeme tun, kann ohne Einschränkung der Allgemeinheit in der Fernfeld- oder Fraunhofer-Näherung betrachtet werden. Für inkohärente, quasi-monochromatischen Quellen kann gezeigt werden (Born \& Wolf 1999), dass die zugehörige PSF durch $P S F(\boldsymbol{\alpha})=|\mathcal{F}[\psi(\boldsymbol{x})](\lambda \boldsymbol{\kappa})|^{2}$ beschrieben werden kann, wobei $\mathcal{F}$ die Fouriertransformation bezeichnet und $\psi$ die komplexe Transmissionsfunktion des kombinierten Systems aus Atmosphäre und Teleskop. Im isoplanaren Fall schreiben wir $\psi=P e^{i \phi}$ mit den reellwertigen Variablen $\phi$ für die Phase und der Apodisationsfunktion $\mathrm{P}$, die normalerweise mittels $\iint P^{2}\left(\boldsymbol{x}_{1}\right) \mathrm{d} \boldsymbol{x}_{1}=1$ normalisiert wird. Als Gaussverteilte Zufallsvariable mit dem Mittelwert Null gilt für die Phase:

$$
\langle\exp (i \phi)\rangle=\exp \left(-\frac{1}{2}\left\langle\phi^{2}\right\rangle\right)
$$



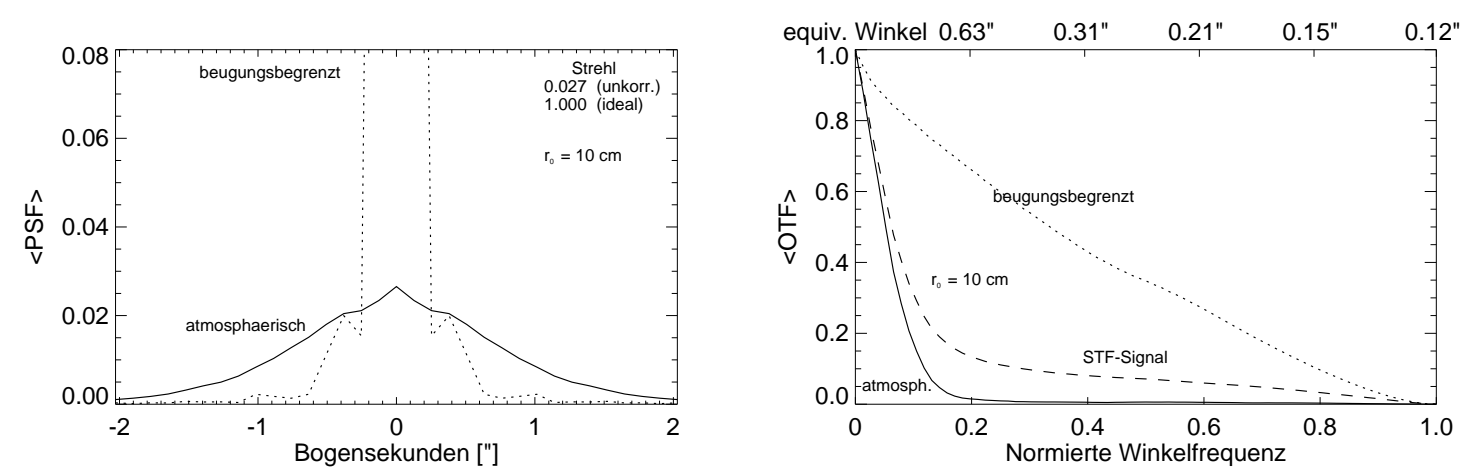

Abbildung 3.2: Beispiel für die Wirkung der Atmosphäre auf die Bildqualität. Links ist die PSF für gewöhnliche atmosphärische Bedingungen $\left(r_{0}=10 \mathrm{~cm}\right)$ zusammen mit der entsprechenden beugungsbegrenzten PSF gezeichnet, deren Spitze beim Wert Eins liegt. Rechts sind die entsprechenden Übertragungsfunktionen gezeigt, zusammen mit dem Fall einer mittleren Kurzzeitbelichtung ('STF'). Hier ist interessant, dass sich beim letzteren Fall ein Plateau ausbildet, das bis zur Auflösungsgrenze reicht, während es bei langen Belichtungszeiten verschwindet ('atmosph.'). Aus kurzen Schnappschüssen durch die Atmosphäre lassen sich also Informationen über sehr viel feinere Einzelheiten des Objektes gewinnen, als bei längeren Belichtungen.

Bringen wir nun mit dem Wiener-Khinchine-Theorem die Langzeitübertragungsfunktion $\mathrm{OTF}_{\mathrm{LE}}(s)$ mit der PSF in Beziehung, können wir die Strukturfunktion $\mathcal{D}$ im Exponenten identifizieren:

$$
O T F_{L E}(\boldsymbol{s})=\iint\left\langle\psi\left(\boldsymbol{s}^{\prime}\right) \psi\left(\boldsymbol{s}^{\prime}+\lambda \boldsymbol{s}\right)\right\rangle \mathrm{d} \boldsymbol{s}=\underbrace{\exp \left[-\frac{1}{2} \mathcal{D}_{\phi}(\lambda \boldsymbol{s})\right]}_{\text {OTFA }} \underbrace{\iint P\left(\boldsymbol{s}^{\prime}\right) P\left(\boldsymbol{s}^{\prime}+\lambda \boldsymbol{s}\right) \mathrm{d} \boldsymbol{s}^{\prime}}_{\text {OTF }} .
$$

Dabei führt die Isoplanatizität von $\psi$ zur Homogenität von $\mathcal{D}_{\phi}$. Die Funktionen $\mathrm{OTF}_{\mathrm{A}}$ bzw. $\mathrm{OTF}_{\mathrm{T}}$ sind Transferfunktionen die zur Beschreibung der atmosphärischen Phasenfluktuationen bzw. zum (beugungsbegrenzten) Teleskop gehören. Sie beschreiben ausschließlich Phase und Amplitude von $\phi$, solange wir uns im Nahfeld-Regime bewegen, wo atmosphärische Szintillation eine vernachlässigbare Rolle spielt. Roddier (1981) leitet für diesen Fall $\mathrm{OTF}_{\mathrm{A}}$ und $\mathcal{D}_{\phi}$ für die integrierte Phasenverzögerung entlang der Sichtlinie ab und erhält als Übertragungsfunktion in der Nahfeldapproximation

$$
O T F_{A}(s)=\exp \left[-3.44\left(\frac{\lambda s}{r_{0}}\right)^{5 / 3}\right]
$$

und von dieser kann auf die Strukturfunktion in der Form 3.23 geschlossen werden. Beispielhaft sind in Abbildung 3.2 die PSF und OTF für eine kreisförmige Teleskopöffnung, mit Obstruktion durch die Halterung des Sekundärspiegels, unter typischen SeeingBedingungen und für den begungsbegrenzten Fall gezeigt. 


\subsubsection{Langzeit- und Kurzzeitbelichtung}

In erster Näherung werden zunächst nur geometrisch optische Effekte untersucht, d.h. die Beugung einlaufender Wellenfronten an Turbulenzstrukturen wird vernachlässigt. Nur die differentiellen Phasenretardationen zwischen nebeneinanderliegenden Lichtstrahlen durch lokale Brechungsindexfluktuationen werden also betrachtet und Szintillation soll keine Rolle spielen (Nahfeldapproximation, siehe dazu Fried (1966a) und Roddier(1981)). Für exzellente Beobachtungsstandorte beschreibt diese Näherung bereits den allergrößten Anteil der Bilddegradationen durch die Atmosphäre.

Wie oben angeführt, beschreibt die OTF im isoplanaren Fall die Abbildungseigenschaften eines astronomischen Teleskops vollständig. Muss durch ein turbulentes Medium beobachtet werden, setzt sich die Gesamtübertragungsfunktion $\left(\mathrm{OTF}_{\mathrm{A}}(s)\right)$ aus dem Produkt eines Teleskopanteils $\left(\mathrm{OTF}_{\mathrm{T}}(\boldsymbol{s})\right)$ und des atmosphärischen Anteils $\left(\mathrm{OTF}_{\mathrm{A}}(\boldsymbol{s})\right)$ zusammen und wurde von Roddier (1981) als

$$
O T F_{A}(\boldsymbol{s})=\left\langle\exp \left(i\left[\phi\left(\boldsymbol{s}^{\prime}\right)-\phi\left(\boldsymbol{s}^{\prime}+\lambda \boldsymbol{s}\right)\right]\right)\right\rangle=\exp \left[-\frac{1}{2} D_{\phi}(\lambda \boldsymbol{s})\right]
$$

definiert. $\mathrm{OTF}_{\mathrm{T}}(\boldsymbol{s})$ ist einfach die Autokorrelationsfunktion der Pupillenfunktion des Teleskops. Die PSF des kombinierten Systems Atmosphäre-Teleskop ist dann durch die Fouriertransformation der Gesamt-OTF

$$
O T F_{L E}(s)=O T F_{A}(s) \cdot O T F_{T}(s)
$$

gegeben. Die Größe $D_{\phi}$ in Gleichung 3.27 muss allerdings nicht exakt wie in Gleichung 3.21 definiert aussehen. Insbesondere im Fall einer partiellen Korrektur der Phasenunterschiede durch Adaptive Optik wird die Phasenstrukturfunktion modifiziert und in der neuen Form in 3.27 eingesetzt. Für Kolmogorov'sche Turbulenz zeigt Abb. 3.2 den Effekt auf die Abbildung bei langer Belichtungszeit. Die OTF fällt sehr viel schneller auf Null (bzw. unter das Rauschniveau) als im beugungsbegrenzten Fall, was sich in einer Filterung der hohen Frequenzen ausdrückt. Im Ortsraum wird dies durch die zugehörige abgeflachte, Gausskurvenförmige PSF mit stark erhöhter FWHM bemerkbar.

Beträgt nun die Belichtungszeit nur wenige Millisekunden, liegt eine weitgehend 'eingefrorene' Turbulenzkonfiguration vor und die Ensemblemittelung in G1. 3.27findet keine Anwendung. In diesem Fall hängt die jeweiligen Gestalt der PSF von der momentanen Konfiguration der Turbulenz und der daraus folgenden Verteilung der Phasendifferenzen über die Eintrittspupille des Teleskops ab. Dies führt zu einer OTF, die auch hohe Frequenzen bis zum Beugungslimit überträgt. Dieser Umstand wird beim Speckle imaging (Labeyrie 1970) ausgenutzt.

\subsubsection{Bildschärfe - Strehl'sche Definitionshelligkeit}

Abbildung 3.2 dient auch zur Einführung einer anderen sehr nützlichen und wichtigen Größe, die zur Charakterisierung der Abbildungsleistung eines Systems sehr oft benützt wird, der Strehl'schen Definitionshelligkeit oder einfacher 'Strehl-Zahl' bzw. 'Strehl ratio' (Strehl 1902). Sie ist definiert durch das Verhältnis der Maximalintensität $I_{\text {real }}$ der tatsächlich vorliegenden PSF zur Maximalintensität $I_{\text {ideal }}$ der theoretischen, d.h. beugungsbegrenzten, PSF:

$$
S=\frac{I_{\text {real }}}{I_{\text {ideal }}}
$$


Ist die Pupillenfunktion $\Phi(\rho, \theta)$ eines optischen Systems mit seinen Aberrationen bekannt, lässt sich die Strehl-Zahl nach (Hardy|1998, Kap. 4.3.3) durch Auswertung des Integrals

$$
S=\frac{1}{\pi^{2}}\left|\int_{0}^{1} \int_{0}^{2 \pi} e^{i k \phi(\rho, \theta)} \rho \mathrm{d} \rho \mathrm{d} \theta\right|^{2}
$$

berechnen. Allerdings ist die explizite Form von $\Phi$ nur in Simulationen bekannt. In der Realität der astronomischen Beobachtungen mit oder ohne Kompensation sind die Phasenfluktuationen bestenfalls statistisch bekannt. Man hat aber mehrere Vereinfachungen der Formel 3.30 gefunden, von denen die (erweiterte) Maréchal-Näherung

$$
S \approx e^{-\left(\sigma_{p}\right)^{2}}
$$

die am weitesten verbreitete ist. $\sigma_{p}$ bezeichnet hier die Standardabweichung der Phasenfluktuationen innerhalb der Apertur. Gleichung 3.31 ist für kleinere Phasenfehler bis zu 2 rad brauchbar. Für stärker beeinträchtigte Wellenfronten, wie es bei unkorrigierter atmosphärischer Turbulenz die Regel ist, wird die Struktur eines Bildes bzw. seiner PSF radikaler verändert und das Problem muss auf andere Art angegangen werden.

\subsubsection{Modale Repräsentation von Atmosphärischen Störungen}

Eine kontinuierliche analytische Funktion kann in eine Superposition von orthogonalen Polynomen oder Moden zerlegt werden. Dies gilt in der Folge auch für eine beliebige Phasenfunktion $\phi$, die nach

$$
\phi(\boldsymbol{x}, t)=\sum_{j=1}^{n} a_{j}(t) Z_{j}(\boldsymbol{x})=\mathcal{Z} \boldsymbol{a}
$$

in $n$ Moden entwickelt wird, wobei $\boldsymbol{a}$ der zeitabhängige, n-elementige Spaltenvektor der Entwicklungskoeffizienten $a_{j}$ ist und $\mathcal{Z}$ ein Zeilenvektor, dessen Elemente $Z_{j}$ die zweidimensionalen Basisfunktionen darstellen. Ein bezüglich der Repräsentation von Aberrationen in optischen Systemen mit Kreissymmetrie wohlbekanntes, vollständiges Funktionensystem zur Darstellung von Wellenfrontdeformationen sind die Zernike-Polynome

$$
Z_{j}^{m n}(r, \theta)=R_{m n}(r) \Theta_{m n}(\theta)
$$

mit der Radialfunktion $R_{m n}$ und der Winkelfunktion $\Theta$, die durch

$$
\begin{aligned}
& R_{m n}(r)=\sum_{q=0}^{(n-m) / 2} \frac{(-1)^{2}}{q !} \frac{(n-q) ! r^{n-2 q}}{[(n+m) / 2-q] ![(n-m) / 2-q] !} \quad \text { und } \\
& \Theta_{m n}(\theta)=\sqrt{n+1} \begin{cases}1 & \text { für } m=0 \\
\sqrt{2} \cos (m \theta) & \text { für } m \neq 0, \quad j \text { gerade } \\
\sqrt{2} \sin (m \theta) & \text { für } m \neq 0, \quad j \text { ungerade }\end{cases}
\end{aligned}
$$

mit den Bedingungen, dass $m \leq n$ und $n-m$ gerade sein müssen. Die $Z_{j}$ sind orthogonal und auf die Varianz 1 über die Fläche $\Omega$ des Einheitskreises normiert:

$$
\iint_{\Omega} w(\boldsymbol{x}) Z_{i}(\boldsymbol{x}) Z_{j}(\boldsymbol{x}) \mathrm{d} \boldsymbol{x}=\delta_{i j}, \quad w(\boldsymbol{x})= \begin{cases}\frac{1}{\boldsymbol{x}}, & \boldsymbol{x} \in \Omega \\ 0, & \text { sonst }\end{cases}
$$




\begin{tabular}{|l|l|}
\hline Zernike-Polynom & entsprechende Aberration(en) \\
\hline \hline$Z_{1}=1$ & Piston (konstanter Phasen-Offset) \\
\hline$Z_{2}=2 r \cos \theta$ & Tip und Tilt (laterale Verschiebung) \\
$Z_{3}=2 r \sin \theta$ & Defokus + Piston (longitudinale Verschiebung) \\
\hline$Z_{4}=\sqrt{3}\left(2 r^{2}-1\right)$ & Astigmatismus (45 und $\left.90^{\circ}\right)$ \\
\hline$Z_{6}=\sqrt{6} r^{2} \cos 2 \theta$ & \\
$Z_{5}=\sqrt{6} r^{2} \sin 2 \theta$ & Koma + Tip bzw. Tilt \\
\hline$Z_{8}=\sqrt{8}\left(r^{3}-2 r\right) \cos \theta$ & \\
$Z_{7}=\sqrt{8}\left(r^{3}-2 r\right) \sin \theta$ & 'Trifoil' \\
\hline$Z_{10}=\sqrt{8} r^{3} \cos 3 \theta$ & \\
$Z_{9}=\sqrt{8} r^{3} \sin 3 \theta$ & Sphärische Aberration + Defokus + Piston \\
\hline$Z_{11}=\sqrt{5}\left(6 r^{4}-6 r^{2}+1\right)$ &
\end{tabular}

Tabelle 3.1: Die ersten Zernike-Polynome haben eine enge Verbindung zu klassischen Abbildungsfehlern. Die Darstellung benützt Polarkoordinaten $(r, \theta)$ und die fortlaufende Indizierung $Z_{i}$ der Moden nach Noll (1976).

Die ersten Moden $Z_{j}$ sind von besonderer Bedeutung, da sie sich mit traditionell wohlbekannten Abbildungsfehlern identifizieren lassen (vgl. Tabelle 3.1). Einer der wichtigsten Gründe für die Verwendung von Zernike-Polynomen liegt in der relativ einfachen Form, die es unter Anderem erlaubt, analytische Ausdrücke für die Fouriertransformierten von Filterfunktionen in der Aperturebene hinzuschreiben.

Noll (1976) stellte eine sehr parktische und oft gebrauchte modale Beschreibung der Kolmogorov'schen Turbulenz bereit, indem er die statistischen Gewichte der Projektionen auf die Zernike-Moden berechnete. Wang \& Markey (1978) benutzten dann diese Ergebnisse, um OTFs für die turbulente Atmosphäre zu berechnen. Später erweitert Winker (1991) die Zernike-Zerlegung, um eine endliche 'outer scale' zu berücksichtigen, und Roddier et al.(1993) leiteten statistische Eigenschaften des zeitlichen und winkelabhängigen Verhaltens der Zernike-Moden unter der Annahme von Kolmogorov'scher Turbulenz ab. Allerdings sind Zernike-Moden nicht die optimale Basis für eine modale Projektion von Kolmogorov'scher Turbulenz: Wie Noll (1976) zeigen konnte, enthält die Kovarianzmatrix $C_{a}=\left\langle\boldsymbol{a} \boldsymbol{a}^{T}\right\rangle$ Nicht-Diagonalelemente, die bedeuten, dass die Zernike-Moden statistisch miteinander korreliert sind. Dies bedeutet, dass es eine effizientere ModenZerlegung geben muss, die durch eine Diagonalisierung von $C_{a}$ durch eine unitäre Matrix $U$ gefunden werden kann. Definiert man den Basisvektor dieser optimalen Zerlegung gemäß $\boldsymbol{k}=U^{T} \boldsymbol{a}$, erhalten wir die neue, diagonale Kovarianzmatrix $C_{k}=U^{T} C_{a} U$. Diese Zerlegung ist als Karhunen-Loéve Basis (KL-Moden) bekannt. Weitere Modifikationen sind insbesondere für den Fall einer Zentralabschattung möglich, wobei die Abweichun- 
gen erst bei substanziellen Abschattungen merklich werden. Auch die KL-Moden können natürlich durch die Zernike-Moden ausgedrückt werden. Glücklicherweise ist diese Zerlegung für die niedrigeren Moden recht kompakt, sodass einerseits mit der optimalen Modenzerlegung gearbeitet werden kann, jedoch nicht auf die analytische Berechnung verzichtet werden muss. Dies erweist sich gerade für die modale Wellenfrontkorrektur mit deformierbaren adaptiven Spiegeln als nützlich (Scharmer et al. 2003a|b), da diese durch ihr Design auf natürliche Art azimutale Sinus nachbilden, wie sie bei Zernikeund Karhunen-Loéve-Funktionen vorkommen (siehe Abschnitt 4.1.4.1). Zur Illustration aller besprochenen Moden möge Abbildung 3.3 dienen, die die ersten (modifizierten) KLModen für eine Teleskopöffnung mit einer kleinen Zentralabschattung gezeigt.

\subsubsection{Anisoplanatismus}

Ein anderer wichtiger geometrischer Effekt bei der Abbildung durch eine turbulente Atmosphäre ist der Winkel-Anisoplanatismus. Er ist eine Konsequenz der unterschiedlichen Blickrichtungen im Gesichtsfeld. Lichtstrahlen aus unterschiedlichen Richtungen durchqueren auch unterschiedliche Ausschnitte der Atmosphäre und erfahren, wie in Abb. 3.4 illustriert, jeweils andere Phasenverzögerungen. In Abschnitt 3.4.1 hatten wir aber isoplanatische Bedingungen vorausgesetzt, was bei inertialer Turbulenz nur im statistischen Mittel gilt. Betrachtet man die momentane Konfiguration von dreidimensionalen Turbulenzfluktuationen $n(\boldsymbol{x}, h, t)$ des Brechungsindex, wird klar, dass keine Isoplanasie vorliegen kann: Unter der Nahfeld-Näherung schreibt man dann die Phasenverzögerung entlang der Sichtlinie $\boldsymbol{\theta}$

$$
\phi(\boldsymbol{x}, \boldsymbol{\theta}, t)=k \int_{0}^{\infty} n(\boldsymbol{x}-h \boldsymbol{\theta}, t) \mathrm{d} h .
$$

Definiert man die Achse $\theta=0$ über die Sichtlinie der Referenzquelle, wird die AOKorrektur auf der Messung der Referenzwellenfront $\phi(\boldsymbol{x}, 0, t)$ beruhen. Beobachtungsobjekte in anderen Richtungen werden in der Folge schlechter abgebildet, da die Kohärenz zwischen Referenzwellenfront und tatsächlicher WF $\phi(\boldsymbol{x}, \boldsymbol{\theta}, t)$ abnimmt (vgl. Abb. 3.4. rechts). Dieser Umstand wird in unkompensierten Beobachtungen nur bei sehr kurzen Belichtungszeiten durch bildfeldabhängige Aberrationen bemerkbar; für längere Mittelungszeiten tendiert die Abbildungsqualität zu isotropem Verhalten, wie oben diskutiert. Für AO-unterstützte Beobachtungen stellt die Anisotropie jedoch ein fundamentales Problem dar. Gehen wir von der Strukturfunktion $\mathcal{D}_{\phi}$ in Gleichung 3.21 aus und nehmen $D \gg r_{0}$ an. Dann lässt sich der mittlere quadratische Fehler durch Anisoplanasie $\left\langle\sigma_{\theta}^{2}\right\rangle$ berechnen, indem wir $r=\theta h \sec (\zeta)$ einsetzen:

$$
\left\langle\sigma_{\theta}^{2}\right\rangle=2.91 k^{2}(\sec (\zeta))^{8 / 3} \theta^{5 / 3} \int_{0}^{\infty} C_{n}^{2}(h) h^{5 / 3} \mathrm{~d} h
$$

Ähnlich zur Definition des Fried-Parameters ist es auch hier möglich, eine charakteristische Größe, den isoplanatischen Winkel

$$
\theta_{0}=\left[2.91 k^{2}(\sec (\zeta))^{8 / 3} \int_{0}^{\infty} C_{n}^{2}(h) h^{5 / 3} \mathrm{~d} h\right]^{-3 / 5}
$$




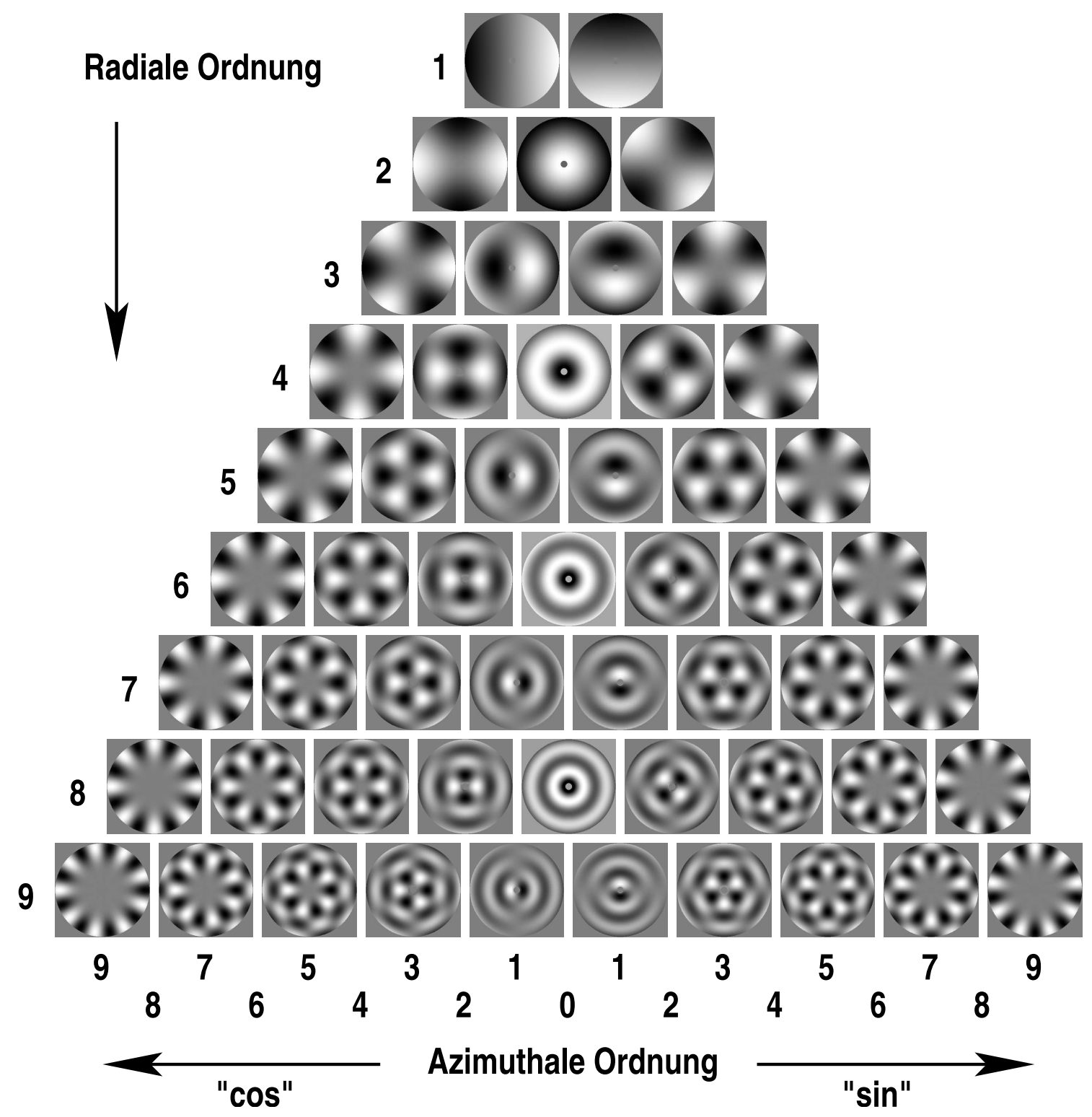

Abbildung 3.3: Die ersten 56 modifizierten Karhunen-Loéve-Moden für eine Teleskopöffnung mit Zentralabschattung (z. B. durch einen Sekundärspiegel). Die Moden bilden auf der Kreisfläche ein orthonormales und statistisch unabhängiges Funktionensystem. Im Sinne der Minimierung der Varianz des Wellenfrontfehlers bilden sie die effizienteste Modenzerlegung einer atmosphärischen Wellenfront.

zu definieren, so dass wir den Anisoplanasiefehler bei Kolmogorov'scher Turbulenz mit Hilfe von $\theta_{0}$ schreiben können (Fried 1982):

$$
\sigma_{\theta}^{2}=\left(\frac{\theta}{\theta_{0}}\right)^{5 / 3} .
$$

Die Interpretation von $\theta_{0}$ entspricht gewissermaßen der von $r_{0}$ und definiert denjenigen Winkelabstand zwischen zwei Quellen im Unendlichen, bei dem die relativen Phasen- 

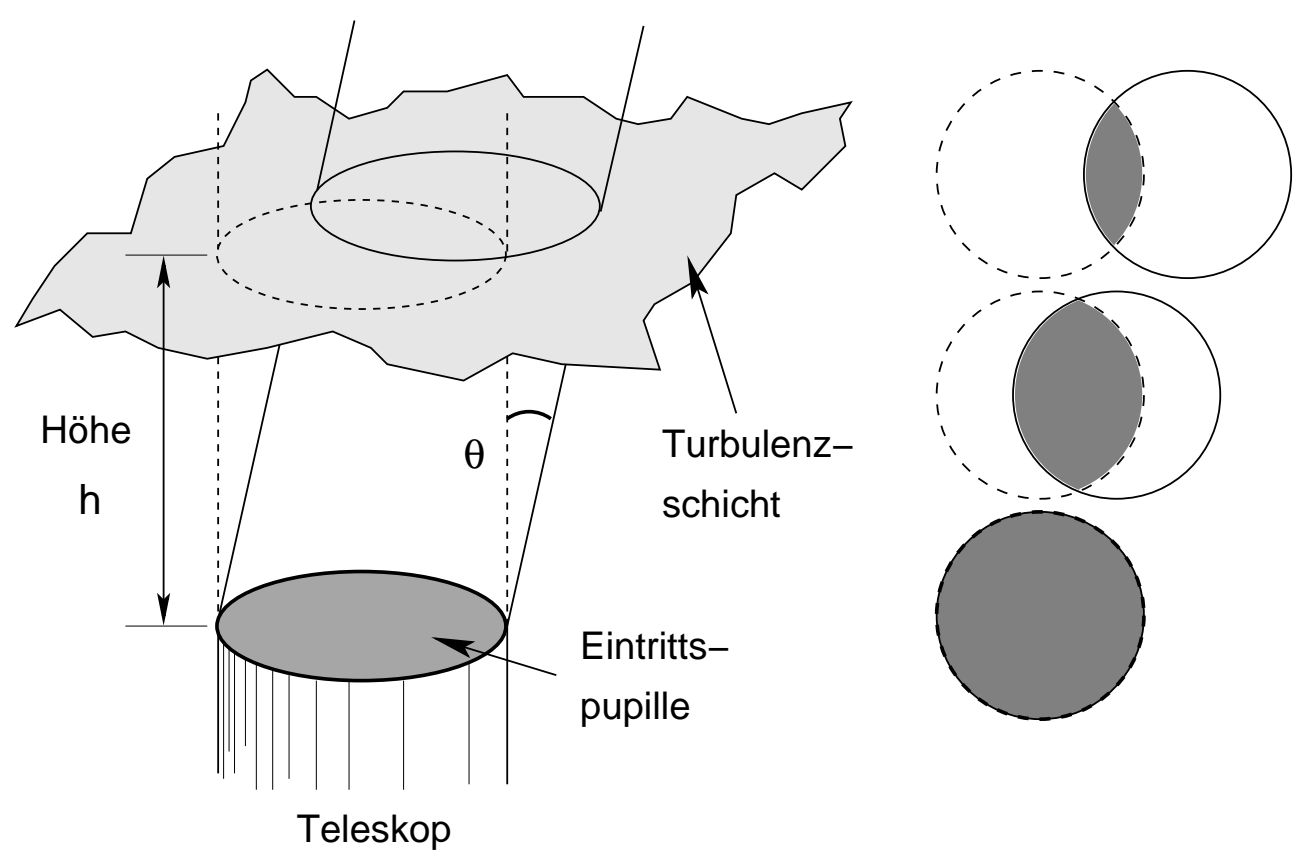

Abbildung 3.4: Illustration zur Ursache des Anisoplanatismus. Nur die skizzierten Ausschnitte der Wellenfronten von der Größe der Eintrittspupille des Teleskops bestimmen, abhängig vom Winkel $\theta$ unter dem sie in das Teleskop treten, die Abbildungsqualität an den Koordinaten $\boldsymbol{\theta} f_{\text {eff }}$ in der Detektorebene. Bei einer hohen Turbulenzschicht tritt ein Versatz $h \cdot \theta$ des jeweiligen WF-Ausschnittes verglichen mit der Referenzwellenfront auf, sodass andere Phasenverzögerungen die Aberrationen des Punktes bestimmen.

differenzen einen rms-Wert von etwa 1 rad aufweisen. Allerdings weist der isoplanatische Winkel eine starke Abhängigkeit von der Vertikalstruktur des Turbulenzprofils auf, wohingegen der Fried-Parameter nur von der über die Sichtlinie aufintegrierten Strukturfunktion abhängt. Wie man intuitiv vermutet, tragen hohe Turbulenzschichten stark zum differentiellen Fehler zweier einfallender Wellenfronten bei, bodennahe Turbulenz hat dagegen praktisch keinen solchen Einfluss und bewirkt nur Abbildungsfehler, die über das Bildfeld konstant sind. Dieser Sachverhalt wird in Abbildung 3.5 deutlich,

Das Potenzgesetz 3.39 beschreibt annähernd auch den Anisoplanatismus in einem Adaptiven Optischen System, obwohl es die begrenzte Übertragungsfähigkeit des Teleskops für hohe Frequenzen vernachlässigt, sowie den partiellen Charakter der Korrektur nicht berücksichtigt. Eine realistischere Abhängigkeit vom Winkel wird für ein System, das nur wenige, niedrige Moden korrigiert, durch ein quadratisches Gesetz $\sigma_{\theta}^{2} \propto \theta^{2}$ beschrieben, wie zuerst von (Roddier et al. 1993) demonstriert wurde.

Die durch den Anisoplanatismus feldabhängige PSF erschwert es nun erheblich, genaue photometrische Aussagen über das abgebildete Objekt oder das Rauschen zu treffen. Auch die Anwendung weiterer Bildrekonstruktionsmethoden, wie etwa Entfaltungsalgorithmen, wird technisch schwierig, da schon die Bestimmung der PSF selbst unsicherer wird. Ein Vorteil der Sonnenbeobachtung ist hierbei gegenüber der Nachtastronomie, dass die wünschenswerte Vergrößerung der Anzahl der Referenzobjekte zur genaueren Bestimmung der PSF keinen zusätzlichen Aufwand erfordert, da gewöhnlich in jeder Rich- 


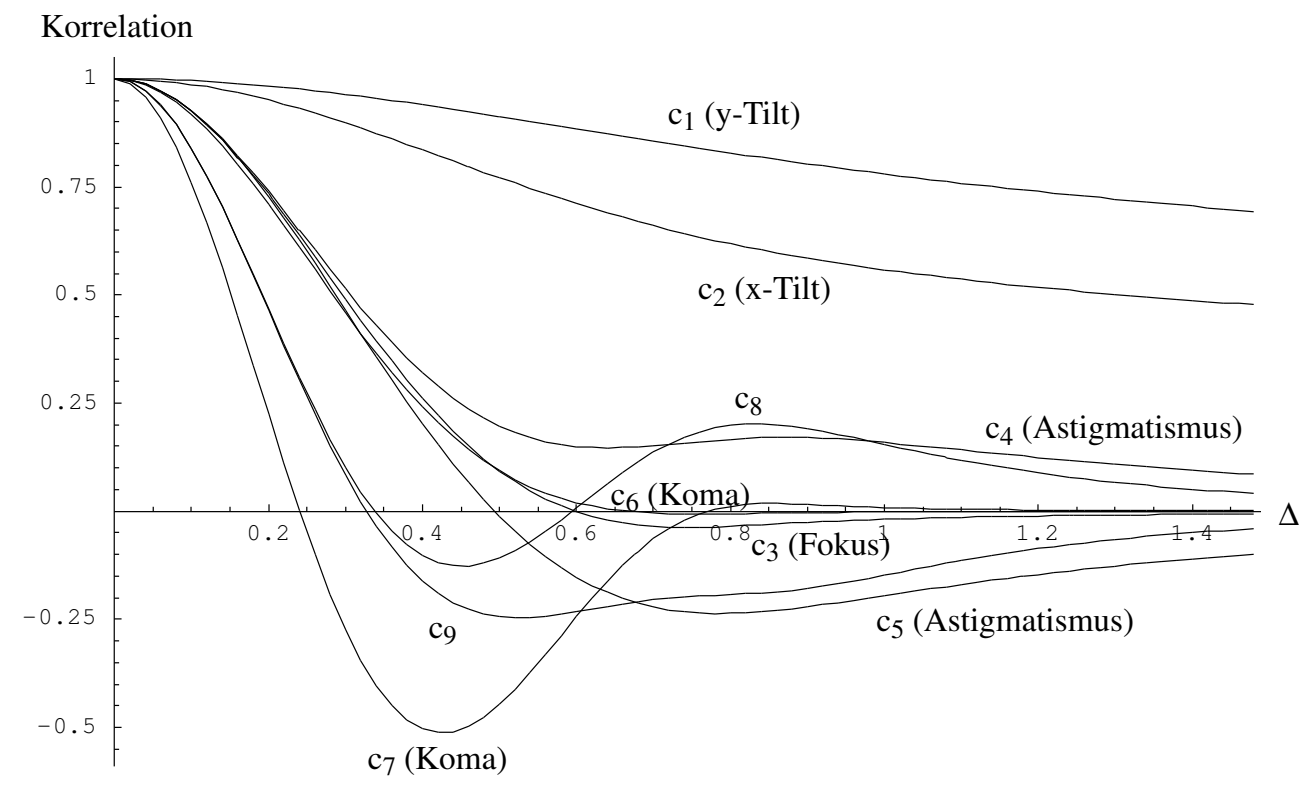

Abbildung 3.5: Korrelationen für verschiedene Zernike-Moden als Funktion des Lichtwegversatzes $\Delta$ in Teleskopdurchmessern $D$. Mit der Höhe $h$ der Turbulenzschicht und dem Winkel $\theta$ zwischen der Sichtlinie und der Referenzrichtung (vgl. Abb. 3.4) ist $\Delta=h \theta / D$. Durch die Wahl der x-Achse parallel zu $\Delta$ ist die Symmetrie der Korrelationskurven gewisser Moden gebrochen, z. B. bei Tip/Tilt oder den beiden Graphen für Astigmatismus. (Graphik entnommen der Dissertation von T. Berkefeld (1998).)

tung ein entsprechender Ausschnitt der Sonnenoberfläche als Referenzobjekt dienen kann. Im Allgemeinen macht es jedoch der durch Adaptive Optik eingeführte Anisoplanatismus deutlich komplizierter und unsicherer, quantitative wissenschaftliche Information aus den Beobachtungen abzuleiten.

\subsubsection{Zeitliche Effekte - Greenwood-Frequenz}

Bis jetzt wurde auf die zeitliche Entwicklung der Turbulenz nur in vereinfachter Form eingegangen, nämlich bei den Ensemble-Mittelwerten der momentanen Realisationen von Phasenstörungen. Unter realen Beobachtungsbedingungen ist dies nicht ausreichend. Gewöhnlich geht man von der Gültigkeit der Taylor-Hypothese des 'frozen flow' aus, die besagt, dass die Struktur der Turbulenz selbst fest sei, während der entsprechende Ausschnitt der Turbulenzschicht vom Wind durch das Gesichtsfeld des Teleskops getragen wird. Diese Annahme wird für Teleskopdurchmesser von bis zu vier Metern gut erfüllt, deckt also den von den Sonnenteleskopen bevölkerten Größenbereich gut ab. Allein die tagsüber stärker ausgebildeten Turbulenzzellen drücken die obere Grenze etwas. Außer für Groß- 
teleskope spielt die zeitliche Entwicklung jedoch eine große Rolle für AO-Systeme, da sie deren minimale Bandbreite bestimmt. Für eine einzelne Schicht, die sich mit einer konstanten Geschwindigkeit $\boldsymbol{v}$ bewegt, kann die Taylor-Hypothese formal in der Form

$$
\phi(\boldsymbol{x}, t+\delta t)=\phi(\boldsymbol{x}-\boldsymbol{v} \delta t, t)
$$

geschrieben werden, was auf eine zeitliche Phasenstrukturfunktion

$$
\mathcal{D}_{\phi}(\boldsymbol{v}, t)=\left\langle|\phi(\boldsymbol{x}, t)-\phi(\boldsymbol{x}-\boldsymbol{v} \delta t, t)|^{2}\right\rangle
$$

führt. Die Gleichung übersetzt die zeitliche Entwicklung in räumliche Entfernung. Man beachte auch, dass dies, abhängig von der Windrichtung, eine Anisotropie in die Phasenstrukturfunktion einführt! Sie wird in zwei interessanten Fällen wieder vernachlässigbar: Bei so langen Zeiten, dass sich alle Korrelationen, die auf der charakteristischen Zeitskala des Mediums bestehen (einige $10 \mathrm{~ms}$ ), herausmitteln, und bei Speckle-Aufnahmen, wo genügend kurze Belichtungszeiten durch die Greenwood-Frequenz $f_{G}$ (Greenwood 1977) charakterisiert werden kann. Diese wichtige Größe ist das Standardmaß für die benötigte Bandbreite bei der Messung atmosphärischer Wellenfrontdistorsionen. Für eine einzelne Turbulenzschicht gilt $f_{G}=0.43|\boldsymbol{v}| / r_{0}$, während für ein Turbulenzprofil $f_{G}$ zu berechnen ist:

$$
f_{G}=\left[0.102 k^{2} \sec (z) \int_{0}^{\infty} C_{n}^{2}(h)|\boldsymbol{v}(h)|^{5 / 3} \mathrm{~d} h\right]^{3 / 5} .
$$

Zusätzlich zur Höhenverteilung $C_{n}^{2}$ der Turbulenzstärke muss dazu auch das vertikale Profil für die Windgeschwindigkeit $\boldsymbol{v}(h)$ bekannt sein (Greenwood 1976). Der Fehler, den allein eine zeitliche Verzögerung $\tau_{0}=f_{G}^{-1}$ verursacht, also wenn die AO 'perfekt' korrigiert, ist mit der Varianz der Phase der Wellenfront ausgedrückt nach Hardy (1998, Gl.9.57)

$$
\sigma_{z e i t}^{2}=28.4\left(\frac{\tau}{\tau_{0}}\right)^{-5 / 3}
$$





\section{Adaptive Optik}

\subsection{Funktionsweise einer Adaptiven Optik}

Nachdem wir die Ursachen der Bilddistorsionen in der Atmosphäre betrachtet haben, wenden wir uns nun der Lösung einiger ihrer Symptome zu, die eine Adaptive Optik (AO) ermöglicht. Zunächst werfen wir einen Blick auf den prizipiellen Aufbau eines Systems, das pupillenkonjugiert arbeitet. Danach wird die modale Kontrolle der Turbulenzkorrektur beschrieben, die im Gegensatz zur zonalen Korrektur für die allermeisten modernen AOSysteme verwendet wird. Es schließt sich ein näherer Blick auf die Systemkomponenten an.

\subsubsection{Allgemeiner Aufbau und wichtigste Elemente}

Die Idee hinter einer Adaptiven Optik ist die Korrektur von optischer Weglängenunterschiede in der Lichtwellenfront eines Objekts, die durch atmosphärische Turbulenz in das Abbildungssystem eingeführt wurden. Dazu müssen die resultierenden Phasendifferenzen zwischen der tatsächlichen Wellenfront (WF) und einer idealen, ebenen Welle zunächst einmal durch einen Wellenfrontsensor (WFS) gemessen werden. Die Korrektur selbst geschieht mittels eines deformierbaren Spiegels. Um seine Oberfläche jedoch in die gewünschte Form zu bringen, müssen die WFS-Messungen in Steuersignale umgerechnet werden, wozu ein sehr schnelles Kontrollsystem benötigt wird. Das heutzutage am meisten verbreitete und vielleicht einfachste Konzept ist das der pupillenkonjugierten AO. Bei ihm wird die Phase der WF an der (Eintritts-)Pupillenebene bestimmt und korrigiert, allerdings wird aus praktischen Gründen in einer optisch konjugierten Ebene im Lichtweg gemessen sowie korrigiert. In Abbildung 4.1 ist schematisch ein solcher optischer Aufbau gezeigt. Eine verzerrte WF tritt in das Teleskop ein und wird relativ zum Aperturdurchmesser verkleinert, d. h. das durch die Eintrittspupille begrenzte Strahlbündel wird auf einen Durchmesser von wenigen cm kollimiert. Über seinen Querschnitt weist das Bündel in der Bildebene der Pupille dann die gleichen Phasenverzögerungen auf. An dieser Stelle trifft die WF dann auf einen deformierbaren Spiegel (DM), der (im idealen Fall) die Form der WF mit halber Auslenkungsamplitude angenommen hat. Nach der Reflexion hat jeder Lichtstrahl im Querschnitt des Strahlenbündels eine (unterschiedliche) Weglänge durchlaufen, sodass die Fläche konstanter Phase, die WF, wieder eben wird. Auf seinem weiteren Weg wird das korrigierte Lichtbündel von einem Strahlteiler aufgeteilt. Der größte Teil des Lichtes wird auf den Detektor abgebildet, wo die Bilddaten für die wissenschaftliche Weiterverarbeitung aufgenommen werden. Der andere Teil des Lichtes wird vom WFS analysiert, der die Abweichungen der WF von einer ebenen 


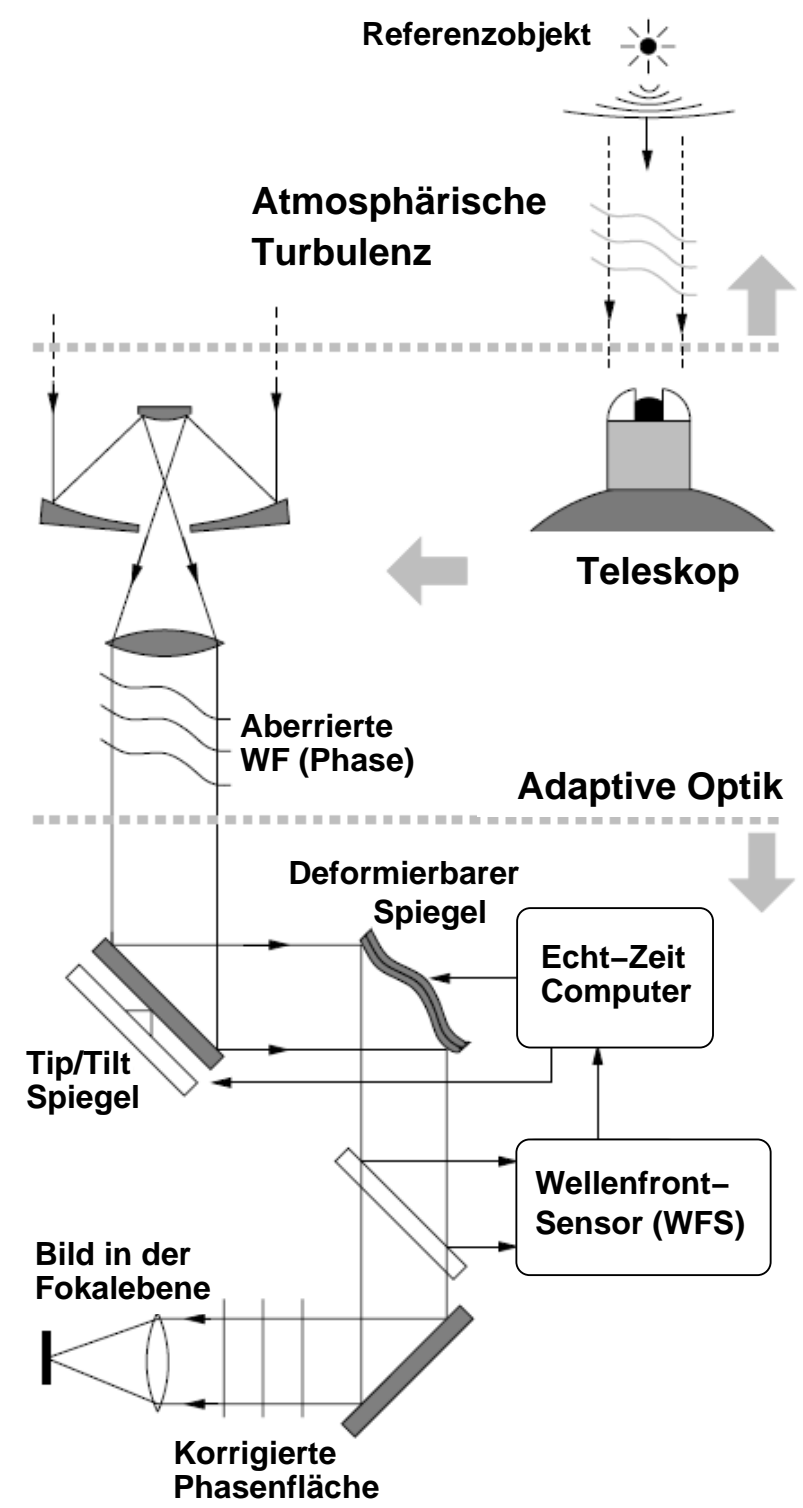

Abbildung 4.1: Skizze des prinzipiellen Aufbaus einer adaptiven Optik. Die Hauptkomponenten bestehen aus dem Wellenfrontsensor (WFS), dem deformierbaren Spiegel (DM), evtl. einem zusätzlichen Tip-Tilt-Spiegel (TT) und Echtzeit-Kontrollsystem.

Welle registriert. Der WFS ist über ein Echtzeit-Kontrollsystem ('real time control', RTC) mit dem DM verbunden. Die gemessenen WF-Abweichungen werden durch das RTC in Kontrollsignale für den DM umgerechnet, um letzteren auf die optimale Form zu 'adaptieren'. Man kann statt einem Strahlteiler auch einen dichroitischen Spiegel verwenden, der es ermöglicht, die WF in einer anderen Wellenlänge zu messen als in der der eigentlichen Beobachtung. Dieser Ansatz maximiert die für den WFS verfügbare Photonenanzahl, einem kritischen Punkt in der Nachtastronomie. Allerdings kann dies auch in der Sonnenbeobachtung relevant werden, insbesondere bei Objekten mit geringem Kontrast. 

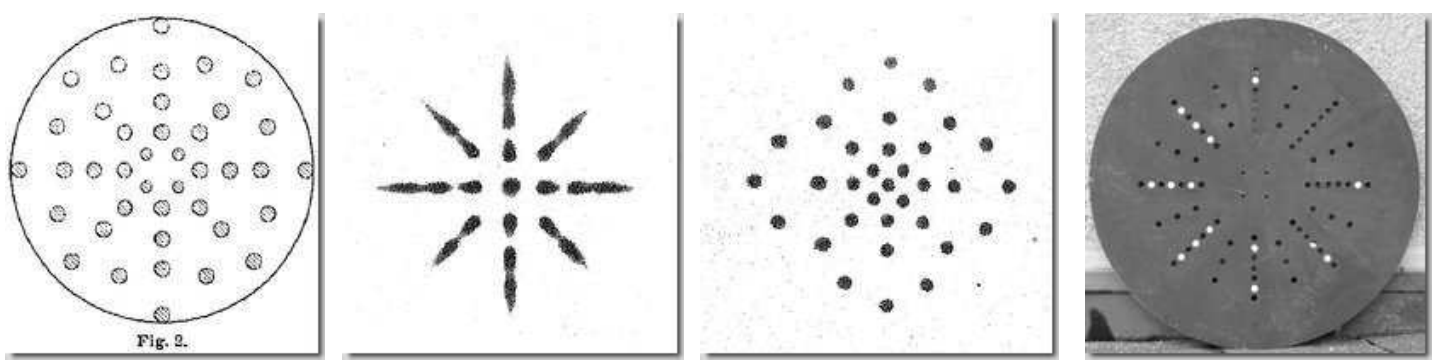

Abbildung 4.2: Zum Prinzip des Hartmann-Tests zur Vermessung des Strahlengangs im Teleskop. Links: Hartmanns Lochblende, ein typisches defokussiertes Bild auf der Fotoplatte und ein Bild, wie es ein Objektiv liefert, das keine Farbfehler aufweist - hier erzeugt durch monochromatisches Licht (Aus: Hartmann (1904)). Rechts: Hartmann-Blende des 70-cm-Spiegelteleskops am Astronomischen Institut Potsdam (AIP).

\subsubsection{Wellenfrontmessung - WFS}

Phasenaberrationen können nur korrigiert werden, wenn eine effiziente Methode für deren Messung gefunden werden kann. Dabei ist es nicht möglich, die Phase der WF bei optischen Wellenlängen direkt zu messen, da bei diesen Frequenzen die nötigen Reaktionszeiten von keinem heute existierenden Detektor erreicht werden. Vielmehr misst man die Intensität des Lichtes und zieht indirekte Methoden heran, um Phaseninformation in messbare Intensitätssignale zu übersetzen. Während man beispielsweise bei der 'phase diversity'-Technik Phaseninformation aus der Intensitätsverteilung im Fokusraum ableitet, werden wir uns hier auf die Pupillenebene konzentrieren, denn die beliebtesten Methoden zur WF-Messung für AO kommen aus dem Gebiet der optischen Prüfung. Verschiedene Typen von Sensoren sind dafür entwickelt worden. Dieser Abschnitt wird sich auf den Shack-Hartmann-Sensor (SHS) konzentrieren, der auf Grund seiner Einfachheit die größte Verbreitung gefunden hat und auch im AO-System KAOS implementiert ist. Eine kurze Diskussion verdiente auch der 'Curvature wave front sensors' (CWS) mit seiner möglichen Anwendung in der Sonnenbeobachtung. Die PSF-Rekonstruktion aus WFSDaten wurde zuerst für diese Art von Sensoren entwickelt. Der jüngste Typ von Sensoren, der 'Pyramid sensor' (Ragazzoni \& Farinato 1999) verspricht, die Vorteile von SHS und CWS zu verbinden, hat aber seinen Hauptanwendungsbereich in der Nachtastronomie, deshalb wird hier nicht näher auf ihn eingegangen.

\subsubsection{Shack-Hartmann-Wellenfrontsensor}

Der SHS stellt eine technologische Weiterentwicklung des Hartmann-Tests dar. Dieser war von Johannes Hartmann schon vor über hundert Jahren (Hartmann 1904) zur Messung der Aberrationen des 'Großen Refraktors' in Potsdam benutzt worden. Dazu wird eine Maske (vgl. Abb. 4.2) mit kreisförmigen Löchern hinter eine Linse montiert, die einzelne Lichtbündel aus dem einfallenden Licht auswählt, deren Strahlengang dann vermessen werden kann. Die Analyse des charakteristischen Musters etwas außerhalb der Fokalebene gibt dann Aufschluss über die Abbildungseigenschaften des Objektivs und dient als Maß für die lokale Neigung der WF. Roland Shack verbesserte mit Ben Platt 


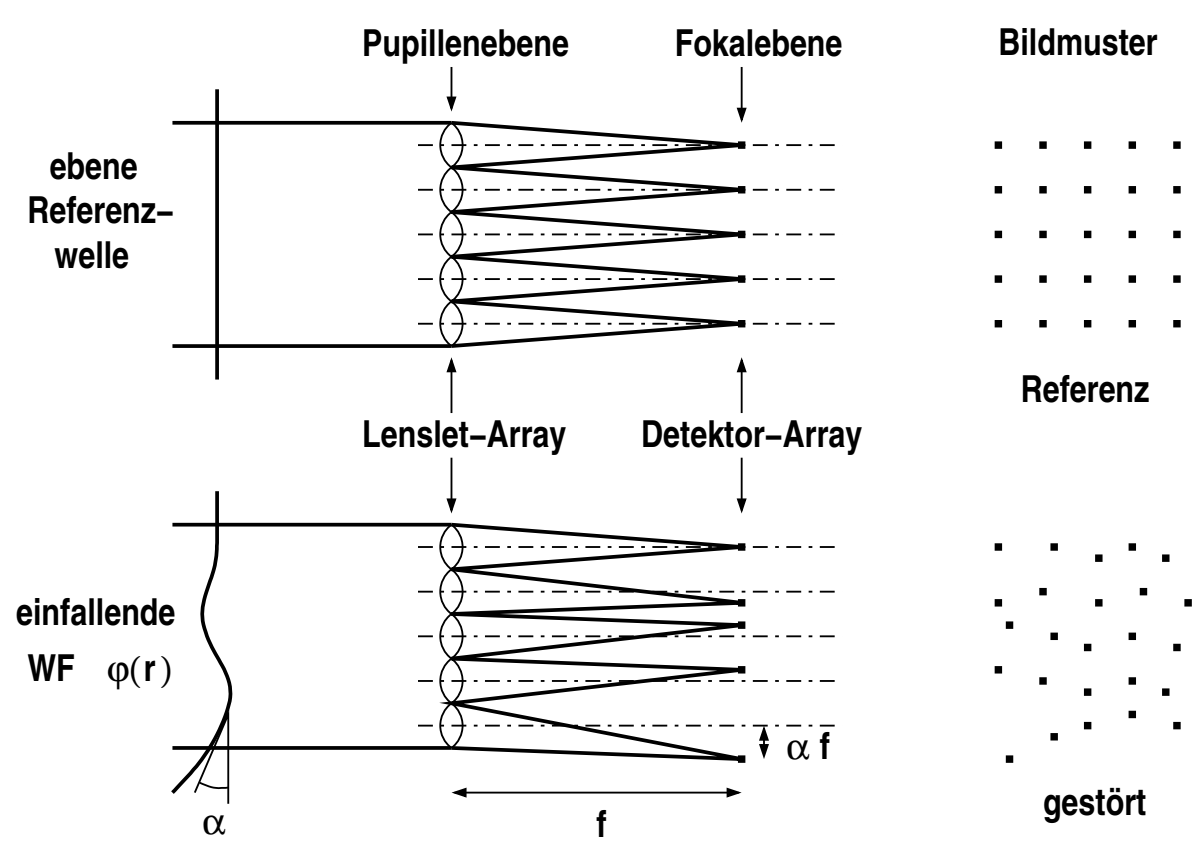

Abbildung 4.3: Zum Prinzip des Shack-Hartmann-Wellenfrontsensors. Oben: Ebene Welle als Referenz; unten: Effekt einer gestörten Wellenfront.

das System (Shack \& Platt 1971) durch die Verwendung von kleinen Linsen ('lenslets') anstelle der Löcher und konnte somit außerdem den gesamten Strahlquerschnitt prüfen. Dies war im Rahmen von militärischen Projekten zur Satellitenbeobachtung für die Registrierung der lichtschwachen Signale von zweifachem Vorteil. Durch die Linsen wird einerseits der Lichtverlust durch die Maskierung vermieden und andererseits die Intensität durch die Linsenwirkung noch zusätzlich erhöht. Abbildung 4.3 zeigt den SHS Aufbau: Am Lenslet-Array wird ein Bild der Eintrittspupille des Teleskops erzeugt. Das Licht des Leitsterns hat dort jedoch parallelen Strahlengang und wird hier durch die zugehörige WF dargestellt. Jedes Lenslet verursacht nun die Entstehung eines Bildes des Leitsterns in der Detektorebene. Im Falle der ebenen WF erhält man entsprechend dem Lensletgitter eine ebenso regelmäßige Anordnung der Bilder auf dem CCD-Chip. Diese Positionen definieren die Referenzpositionen. Dagegen verursacht eine verkrümmte WF nun eine Verschiebung der Bildpunkte, verglichen mit dem unaberrierten Fall. Dabei ist die mittlere Neigung der WF über jedem Lenslet das Maß der Verschiebung. Verschwindet die mittlere Neigung des entsprechenden Wellenfrontausschnittes eines Lenslets, erscheint das zugehörige Bild auch nicht verschoben, verglichen mit seiner Referenzposition, allenfalls unscharf. Andernfalls gilt für die Winkelverschiebung $\alpha_{x}$ in Richtung der X-Achse:

$$
\alpha_{x}=\frac{\Delta x}{f M}=\frac{\lambda}{2 \pi A_{s a}} \iint_{A_{s a}} \frac{\partial \phi}{\partial x} d x d y
$$

Ähnliches gilt entsprechend für die y-Achse. In der Gleichung bezeichnet $A_{s a}$ die Fläche der Subapertur, $\Delta x$ die Bildversetzung in der Fokalebene, $f$ die Brennweite der Lenslets und $M$ ist der Vergrößerungsfaktor zwischen Pupille eines Lenslets und der des Teleskops. Die Gleichung bedeutet auch, dass ein SHS die erste Ableitung der WF an den Lensletpositionen misst und Informationen über konstante Phasenverschiebungen 
(Piston) vollständig verloren gehen. Dieser systematische Fehler der Phase beeinflusst die Bildqualität bei normaler Abbildung nicht, allerdings verursacht er bei der Interferometrie Schwierigkeiten, da die Weglängenunterschiede der Interferometerarme sehr genau aneinander angeglichen werden müssen! In der Nachtastronomie wird die aktuelle Bildverschiebung in der Fokalebene des SHS mit einer Minimalanzahl von vier Detektoren pro Subapertur gemessen ('Quadcells'). Die (Schwerpunkts-)Position der Airy-Figur eines Leitsterns verursacht dann im Allgemeinen ungleiche Intensitäten, die direkt den Einfallswinkel liefern. Dagegen muss bei flächenhaften Objekten zur WF-Messung mit größeren Detektorenarrays gearbeitet werden, da hier Verschiebung von Bildausschnitten einer ausgedehnten, unbekannten Struktur bestimmt werden. Dies ist insbesondere in der solaren Beobachtung der Fall ist. Die errechnete Kreuzkovarianzfunktion der einzelnen Ausschnitte mit dem Bild einer frei wählbaren Referenzsubapertur übernehmen dann die Rolle des Leitstern-Abbildes. Im Falle der CCDs stellen die Schätzungen der Lichtpunktpositionen gewöhnlich eine Modifikation der 'Schwerpunktsschätzung'

$$
\Delta x=\frac{\sum_{i} x_{i} I_{i}}{\sum_{i} I_{i}}
$$

dar, mit Pixel offset $x_{i}$ von der Referenzposition und Intensität $I_{i}$ aller Pixel $i$ innerhalb einer Subapertur. Eine wichtige Eigenschaft des SHS ist seine Wellenlängenunabhängigkeit. Dies sieht man im Ausdruck für die Varianz des Einfallswinkels bei kreisrunder Eintrittsöffnung mit Durchmesser D (Tatarskii, 1971)

$$
\left\langle\alpha_{x}^{2}\right\rangle=0.98 \frac{6.88}{4 \pi^{2}}\left(\frac{\lambda}{D}\right)^{2}\left(\frac{D}{r_{0}}\right)^{5 / 3}=0.171 \lambda^{2} D^{-1 / 3} r_{0}^{-5 / 3} \quad \propto \lambda^{0} D^{-1 / 3}
$$

Die turbulenzinduzierten Unterschiede in der optischen Weglänge ('optical path differences', $O P D$ ) sind wegen $r_{0} \propto \lambda^{6 / 5}$ achromatisch, und da die lokale Verkippung gemessen wird, arbeitet der SHS auch mit breitbandigem Weisslicht bzw. kann immer mit Licht aus spektralen Bereichen betrieben werden, die nicht für die wissenschaftliche Beobachtung benötigt werden. Im Gegensatz zu anderen Sensortypen ist der SHS auch für die Wellenfrontmessung mit ausgedehnten Referenzobjekten geeignet, wenn nur das Gesichtsfeld ('field of view', FOV) dem Objekt und dem Detektor angepasst wird. So kann durch Verwendung einer geeigneten Feldblende in der Fokalebene erreicht werden, dass die Bilder eines Ausschnittes der Granulation der Photosphäre der Sonne vom LensletArray ohne Überlapp auf einen einzigen CCD-Chip projiziert werden. Dieser Ausschnitt ist nicht auf den isoplanaren Bereich beschränkt. Gerade für Erweiterungen der Adaptiven Optik wie die Multikonjugierte AO (MCAO) wird ein anisoplanares FOV auf dem CCD benötigt, um die Wellenfrontkorrektur dorthin auszudehnen. Auch hier wurde demonstriert, wie der SHS erfolgreich dazu benützt werden kann, die WF zu messen (Acton \& Smithson 1992) und um beugungsbegrenzte Bilder zu erhalten (von der Lühe et al. 2002). Allerdings ist dann ein minimaler Kontrast für die Messung nötig (Michau et al. 1992).

\subsubsection{Messgenauigkeit}

Spezielles Augenmerk ist auf die Wahl der Subaperturengröße zu richten, da sie die räumliche Auflösung des Sensors bestimmt. Da der SHS eine gegebene WF über den Bereich 
einer Subapertur gemittelt misst, erkennt der Sensor Aberrationen höherer Ordnung als Tip/Tilt nicht, d. h. Raumfrequenzen oberhalb der Abtastfrequenz des Sensors werden abgeschnitten. Aus diesem Grund sind möglichst kleine Subaperturen wünschenswert, damit residuelle Aberrationen tolerierbar werden. Andererseits bedeutet die Verkleinerung der Subaperturen geringere Beleuchtungsstärken, was in der Nachtastronomie oft kritisch wird und in der solaren Beobachtung zu schlechtem Signal zu Rauschen-Verhältnis führt. Ein zweiter Effekt zu kleiner Subaperturen besteht darin, dass Beugungseffekte die PSF so verbreitern, was die Schärfe der Bilder so herabsetzt, dass dies wieder den Fehler bei der Schätzung der Wellenfrontneigung erhöht. Der optimale Durchmesser einer Subapertur, in die Ebene der Teleskoppupille projiziert, entspricht also in etwa dem Fried-Parameter $r_{0}$ und sollte allenfalls geringfügig kleinere Abmessungen aufweisen.

\subsubsection{Rauschen}

Natürlich sind alle Sensoren auch mit Rauschen behaftet, das zum Einen vom Lichtsignal selbst hervorgerufen wird, aber auch durch weitere Verarbeitung bzw. Auslesen der Daten. Rousset (1993) hat für den Fall der Nachtastronomie gezeigt, dass alle WFS-Typen, die mit Subaperturen arbeiten, eine ähnliche Abhängigkeit vom Photonenrauschen zeigen:

$$
\sigma^{2} \sim \frac{1}{n_{p h}}\left(\frac{\theta d}{\lambda}\right)^{2}
$$

mit der Varianz des Phasenmessfehlers $\sigma^{2}$, dem Durchmesser $d$ der (kreisförmigen) Subapertur und der Anzahl $n_{p h}$ der Photoelektronen je Subapertur und Belichtungsdauer. $\theta$ bezieht sich dabei auf die Winkelausdehnung der Referenzquelle, die entweder durch ihre tatsächliche Größe gegeben ist, oder durch ihre virtuelle Ausdehnung, die durch Einfluss des seeings oder der Beugung bestimmt wird. Da die Größen $d$ und $\lambda$ normalerweise fest sind, kann bei maximaler Effizienz der Photosensoren ein besseres Signal-RauschVerhältnis ('signal to noise ratio', SNR) durch helle, punktförmige Quellen (Leitstern) erreicht werden. Äquivalent dazu werden im Falle der Sonnenbeobachtung für einen höheren Signal-Rausch-Abstand kontrastreiche Strukturen benötigt. Die eigentlichen Messdaten, Bilder von Ausschnitten der Sonnenoberfläche, werden zunächst gegen das Referenzbild kreuzkorreliert. Bei ausreichend hohem Kontrast kann mit den Korrelationsfunktionen im Weiteren wie mit Punktlichtquellen weitergearbeitet werden.

\subsubsection{Wellenfrontrekonstruktion}

Die oben angesprochenen Wellenfrontsensoren messen eine Ableitung der WF. Das Ziel ist aber, diese Messungen in eine Schätzung der eigentlichen WF umzuwandeln, die dann dazu benutzt werden kann, ein Korrekturelement zur Kompensation der Aberrationen zu betreiben. Die Ableitungen der WF sind lineare Funktionen der WF, was es ermöglicht, lineare Gleichungen im Rekonstruktionsprozess zu verwenden, was sich mit Matrixalgebra gut ausdrücken lässt: Die Unbekannte stellt einen Vektor $\phi$ aus n Signalen bzw. Phasenwerten auf einem Gitter dar. Diese müssen aus einem m-elementigen Datenvektor $S$ berechnet werden, der im Falle des SHS die Wellenfrontneigungen in jeweils zwei Richtungen enthält. Folgende allgemeine lineare Beziehung muss erhalten werden:

$$
\phi=B S,
$$


wobei $\boldsymbol{B}$ die sogenannte Rekonstruktionsmatrix definiert. Bei der Wellenfrontrekonstruktion geht es also darum, die Matrix $\boldsymbol{B}$ zu bestimmen. Die zwei bekanntesten Klassen von Techniken, die dies leisten, sind die lokalen und die globalen Methoden der Wellenfrontrekonstruktion.

\subsubsection{Zonaler und Modaler Ansatz}

Bei der zonalen Methode wird die Phase an einer Anzahl von diskreten Punkten bestimmt, die über die Teleskoppupille verteilt sind. Diese Werte stellen wir durch den Vektor $\phi$ dar. Ein lineares Modell des WFS erlaubt es, zwischen dem m-elementigen Vektor $\boldsymbol{S}$ der Messwerte und den $\mathrm{n}$ Werten der einlaufenden Phase im Vektor $\phi$ die Matrixgleichung

$$
S=A \phi
$$

aufzustellen, wobei $\boldsymbol{A}$ Interaktionsmatrix genannt wird. Im Fall eines deformierbaren Spiegels kann die Matrix $\boldsymbol{A}$ leicht gemessen werden. Jede Spalte beinhaltet einfach die Messwerte des WFS, die mit jeweils einem einzelnen Aktuator assoziiert sind, d. h. wenn nur ein Aktuator mit einem Einheitswert für die Spannung angesteuert wird, die anderen Aktuatoren jedoch neutral gehalten werden. Um nun zur Rekonstruktionsmatrix $\boldsymbol{B}$ aus Gleichung $4.5 \mathrm{zu}$ gelangen, muss das inverse Problem gelöst werden, wie also die Phasenwerte der WF mit Hilfe der gemessenen Daten geschätzt werden können. Dieser Punkt wird im nächsten Abschnitt näher behandelt, da dieser Arbeitsschritt gleichermaßen beim modalen Ansatz nötig ist. An dieser Stelle sei aber darauf hingewiesen, dass bei diesem Ansatz die eigentliche WF als Fläche gleicher Phase niemals bestimmt wird. Nur einzelne diskrete Werte werden berechnet. So werden die Einflussfunktionen der Aktuatoren des DM, die die tatsächliche Verformung des Spiegels beschreiben, auch nicht bei der Ableitung der Matrix $\boldsymbol{B}$ explizit benutzt und müssen daher nicht bekannt sein. Da sie aber bei der Messung der Interaktionsmatrix $\boldsymbol{A}$ vollständig berücksichtigt sind, wird die korrigierende Phasenfläche automatisch vom deformierbaren Spiegel selbst synthetisiert.

Beim modalen Ansatz wird die Phase durch Koeffizienten ihrer Entwicklung nach gewissen Basisfunktionen $Z_{i}$ (Moden) beschrieben. Dazu wird eine Beziehung ähnlich $\mathrm{zu} 4.6$ verwendet, die aus den Messwerten des WFS einen Vektor $\phi=\left\{\phi_{i}\right\}$ von Koeffizienten bildet. Dieser zusätzliche Schritt, verglichen mit der zonalen Methode, bringt den Vorteil, dass die Phase

$$
\varphi(\boldsymbol{r})=\sum_{i} \phi_{i} Z_{i}(\boldsymbol{r})
$$

an jedem Punkt $\boldsymbol{r}$ der Pupillenebene bestimmt ist. Der Summationsindex $i$ läuft dabei von 1 bis zur Modenanzahl $n$ der Entwicklung. Für die Wahl der Basisfunktionen kann man sich von der Art des Problems leiten lassen. Gebräuchlich sind die tatsächlichen Einflussfunktionen der Aktuatoren des DM, da diese naturgemäß am besten auf die Steuerungsprobleme zugeschnitten sind.

Die Interaktionsmatrix $\boldsymbol{A}$ kann im modalen Ansatz mit den analytischen Ausdrücken der Moden $Z_{i}(\boldsymbol{r})$ berechnet werden. Beispielsweise gilt für die x-Komponente des Elementes $A_{i j}$ beim SHS

$$
A_{i j}^{x}=\frac{1}{\mathcal{A}_{\text {sa }}} \int_{\text {subaperture } j} \frac{\partial Z_{i}(\boldsymbol{r})}{\partial x}
$$


und ähnlich für die y-Komponente. Häufig werden als Basisfunktionen auch die ZernikePolynome (siehe Abschnitt 3.4.4) verwendet (Noll 1976), theoretisch optimal ist jedoch das System nach Karhunen-Loève (KL-Moden), da sie die statistischen Eigenschaften der Turbulenz berücksichtigen (Wang \& Markey 1978), sodass ihre Kovarianzmatrix diagonal wird. Vom praktischen Standpunkt aus müssen allerdings letztlich immer die mechanischen Spiegeleigenmoden berücksichtigt werden, da es gewöhnlich nicht möglich ist, reine Zernike- oder KL-Moden zu realisieren. Die Spiegelmoden müssen dann natürlich noch orthogonalisiert werden, was durch Minimierung der Phasenvarianz innerhalb der Pupille geschieht. Die maximale Anzahl von errechenbaren Moden wird in jedem Fall durch die Anzahl der Subaperturen des WFS bestimmt, also durch die Anzahl der Freiheitsgrade des AO-Systems.

\subsubsection{Lösung des inversen Problems}

Um im geschlossenen Regelkreis die Phase $\phi$ der WF aus den WFS Messdaten abzuschätzen, muss der Messfehler

$$
\varepsilon_{s}=\|\boldsymbol{S}-\boldsymbol{A} \phi\|^{2}
$$

minimiert werden. || || bezeichnet hier die Norm eines Vektors. Mit dieser Schätzung $\phi$ nach der Methode der kleinsten Quadrate gilt dann

$$
\left(\boldsymbol{A}^{t} \boldsymbol{A}\right) \boldsymbol{\phi}=\boldsymbol{A}^{t} \boldsymbol{S}
$$

wobei $\boldsymbol{A}^{t}$ die transponierte Interaktionsmatrix bezeichnet. Diese Standardlösung kann meist nicht verwendet werden, da sich die Matrix $\boldsymbol{A}^{t} \boldsymbol{A}$ als singulär erweist. Dies ist eine Konsequenz aus der Tatsache, dass die Phase nur bis auf eine Konstante durch ihre Ableitung bestimmt ist; der WFS ist jedoch gegenüber einer WF, die konstant über eine Apertur ist, unempfindlich. Diese Mode (Piston) muss jedoch nicht beachtet werden, da sie auf die Abbildungseigenschaften des Systems keinerlei Einfluss hat. Deshalb kann der Piston-Term, der mit $i=1$ ein Teil jeder Modenentwicklung 4.7 ist, ausgeklammert werden und nur ein Unterraum des Lösungsraumes betrachtet werden. Bei der Matrix $\boldsymbol{A}$ läuft der Index $i$ dann nur von 2 bis n und die Singularität wird vermieden.

Sieht man die Wellenfrontrekonstruktion als inverses Problem, lautet die Relation zwischen gemessenen Daten $S$ und den Unbekannten $\phi$

$$
S=A \phi+n
$$

wobei $\boldsymbol{n}$ ein Vektor mit additivem Rauschen ist. Der Vektor $\phi$ kann hier sowohl die Aktuatorsignale, die zonalen Werte der Phase oder auch die Koeffizienten der modalen Entwicklung enthalten! Die Fragestellung lautet nun, die 'beste' Schätzung für die Unbekannten $\phi$ aus den bekannten Messwerten $\boldsymbol{S}$ abzuleiten, nun jedoch unter Berücksichtigung von a priori bekannten Eigenschaften. Oft ist die Statistik des Rauschens und des Vektors $\phi$ bekannt, aus theoretischen Betrachtungen oder aus den Beobachtungsdaten selbst quantifizierbar. Damit bieten sich zwei Standpunkte für die beste Schätzung: die 'maximum likelihood-' und die 'Maximum a posteriori'-Technik (Sasiela \& Mooney 1985, Fried 1993): 
Im Ersten gilt es, die sogenannte 'likelihood'-Funktion zu maximieren, die direkt durch die Wahrscheinlichkeitsfunktion für $S$ bei bekanntem $\phi$

$$
P(\boldsymbol{S} \mid \boldsymbol{\phi})=\frac{1}{\sqrt{(2 \pi)^{M}\left|\boldsymbol{C}_{n}\right|}} \exp \left[-(\boldsymbol{S}-\boldsymbol{A} \boldsymbol{\phi})^{t} \boldsymbol{C}_{n}^{-1}(\boldsymbol{S}-\boldsymbol{A} \boldsymbol{\phi}) / 2\right]
$$

gegeben ist. Dabei bedeuten $\boldsymbol{C}_{n}$ die Kovarianzmatrix des Rauschens $\boldsymbol{n}$ und $\left|\boldsymbol{C}_{n}\right|$ ihre Determinante. Eine generalisierte Lösung

$$
\phi=\left(\boldsymbol{A}^{t} \boldsymbol{C}_{n}^{-1} \boldsymbol{A}\right)^{-1} \boldsymbol{A}^{t} \boldsymbol{C}_{n}^{-1} \boldsymbol{S}
$$

erhalten Sasiela \& Mooney (1985) durch Maximierung des Logarithmus von 4.12, wobei die Invertierbarkeit von $\boldsymbol{A}^{t} \boldsymbol{C}_{n}^{-1} \boldsymbol{A}$ in jedem Fall zu überprüfen ist. Hat man keine Korrelationen zwischen den Messungen der Subaperturen und liegen keine Abschattungen von Subaperturen durch die Pupille vor, wird $\boldsymbol{C}_{n}$ diagonal und die Verteilung der Varianz des Rauschens gleichmäßig über alle Subaperturen. Dann reduziert sich die Gleichung und wir haben wieder die Standardlösung 4.10 vorliegen. Ein besseres Verhalten gegenüber dem Rauschen zeigt jedoch die a posteriori-Technik. Maximiert die maximum likelihoodMethode die Wahrscheinlichkeit für die Messdaten, wenn ein Modell gegeben ist, drückt diese Technik aus, was man intuitiv möchte: die Maximierung der Wahrscheinlichkeit einer Lösung bei gegebenen Messungen. Dies kann nach dem Bayes'schen Theorem geschrieben werden als

$$
P(\boldsymbol{\phi} \mid \boldsymbol{S})=\frac{P(\boldsymbol{\phi}) P(\boldsymbol{S} \mid \boldsymbol{\phi})}{P(\boldsymbol{S})} .
$$

Da nun die Daten als Voraussetzung genommen sind, gilt $P(\boldsymbol{S})=1$. Die Statistik von $\phi$ ist nun aber a priori-Wissen. Man sieht dann, dass der Unterschied zur maximum likelihood-Methode in der zusätzlichen Minimierung der Wahrscheinlichkeitfunktion $P(\phi)$ besteht. Diese sorgt für die nötige Regularisierung, um Rauschverstärkung bzw. die Unterbestimmung des Gleichungssystems zu überwinden. Einen aktuellen Überblick über Grundlagen und Weiterentwicklungen auf dem Gebiet der Bildrekonstruktion und inversen Probleme findet sich bei Thiébaut (2005).

\subsubsection{Wellenfrontkorrektur}

Der WF-Korrektor ist das Element eines AO-Systems, das zur Kompensation jeglicher Aberrationen, seien sie atmosphärischer oder technisch-optischen Ursprungs, verwendet wird. Seine Aufgabe ist es, die Phasenunterschiede über den Strahlquerschnitt auszugleichen, also entsprechende optische Weglängenunterschiede einzuführen, die die atmosphärisch verursachten möglichst genau kompensieren (Abb. 4.4). Wie in Abb. 4.1 schon angedeutet, besteht der Korrektor gewöhnlich aus zwei Spiegeln, dem ebenen, nicht verformbaren Tip-Tilt-Spiegel (TT) und dem deformierbaren Spiegel (DM). Beide Spiegel werden gewöhnlich durch Anlegen gewisser elektrischer Spannungen gesteuert, die von der WF und von technischen Spezifikationen der Geräte abhängen. Der ebene TT gleicht dabei nur die durchschnittliche WF-Verkippung aus, die durch die beiden Zernike-Moden 'Tip' und 'Tilt' dargestellt wird und schon mit knapp 87\% zur Kolomogorov'schen Phasenvarianz beiträgt. Diese Aufteilung hat vor allem den Vorteil, dass damit den Aktuatoren des DM mehr Hub zur Korrektur der Moden höherer Ordnung bleibt, wenn auch ein gewisser Lichtverlust durch die zusätzliche Reflexion in Kauf genommen werden muss. Zur 


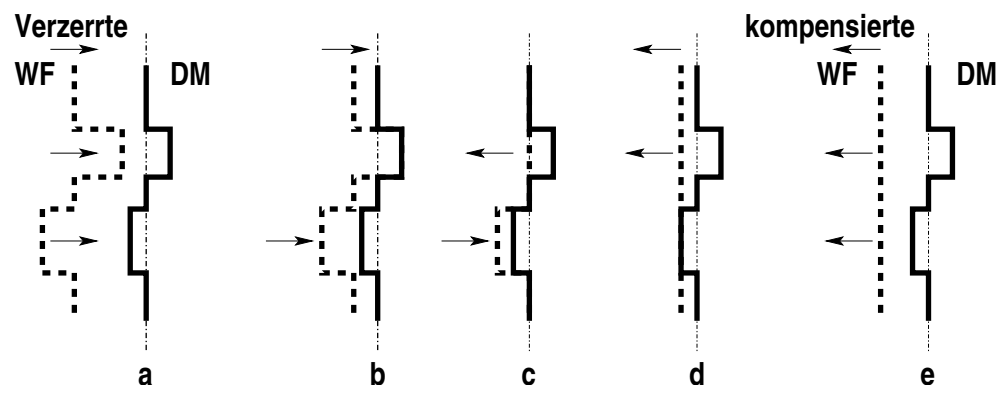

Abbildung 4.4: Prinzip der Phasenkonjugation bei der Korrektur einer Wellenfront durch Adaptive Optik. Die optischen Weglängen werden durch Angleichen der Spiegeloberfläche an die errechnete WF ausgeglichen, wobei zu beachten ist, dass sich die Weglängen durch die Reflexion verdoppeln. Die Spiegeldeformationen haben also nur die halbe Amplitude der WF.

Korrektur der höheren Ordnungen der WF-Deformation wurden anfänglich segmentierte Spiegel verwendet, bei denen z. B. jedes der dichtgepackten Elemente verkippbar und in der Höhe verstellbar war. Beispielsweise wurde ein AO-System mit 19-elementigem Spiegel, das am Sacramento Peak Solar Telescope installiert war (Acton \& Smithson 1992), im Jahr 2000 für experimentelle Messungen, aber auch zur Unterstützung wissenschaftlicher Beobachtungen (Wöger 2001, Sailer 2002) am deutschen VakuumTurmTeleskop auf Teneriffa aufgebaut.

Die Einfachheit und der modulare Aufbau wiegen jedoch die Nachteile nicht auf, von denen der schwerwiegendste der 'fitting error' ist, also der Fehler durch die Differenzen zwischen WF und Spiegelfläche. Außerdem führt die schwer kontrollierbare, unterschiedliche gegenseitige Ausrichtung der Spiegelelemente, verbunden mit der ungenügenden Möglichkeit der Formung einer 'glatten' Fläche, zu Beugungseffekten an den Segmentkanten. Diese Schwierigkeiten werden durch die heutzutage gebräuchlichen kontinuierlichen Spiegel gelöst.

\subsubsection{Bimorphe Spiegel}

Bimorphe Spiegel bestehen aus zwei piezoelektrischen Keramik-Scheiben, den Komponenten $\mathrm{A}$ und $\mathrm{B}$, die parallel zu ihren Achsen verbunden und entgegengesetzt polarisiert werden. Ein Array von Elektroden liegt zwischen den Platten, die an den Außenseiten geerdet werden. Durch Anlegen einer Spannung expandiert A lokal in der lateralen (x,y)Ebene und kontrahiert in der z-Richtung, während B sich gegensätzlich verhält. Die relative Änderung der Länge $l$ ist gegeben durch

$$
\frac{\Delta l}{l}=\frac{V d_{31}}{t},
$$

mit dem transversalen piezoelektrischen Koeffizienten $d_{31}$ und der Dicke $t$ der Keramikscheibe. Diese unterschiedlichen Änderungen verursachen Spannungen und daher eine lokale Krümmung mit dem Radius

$$
R=\frac{t l}{2 \Delta l}=\frac{t^{2}}{2 V d_{31}} .
$$


Nimmt man die lokale Verformung als sphärisch über einen Durchmesser $d$ an, lässt sich die Empfindlichkeit $S_{b}$ als das Verhältnis von mechanischem Hub zu elektrischer Spannung schreiben:

$$
S_{b}=\frac{d^{2}}{8 R V}=\frac{d^{2}}{4 t^{2}} d_{31}
$$

Typische Werte sind etwa $d=40 \mathrm{~mm}, t=1 \mathrm{~mm}$ und $d_{31}=0.2 \mu \mathrm{m} / \mathrm{kV}$, sodass sich $S_{b}=80 \mu \mathrm{m} / \mathrm{kV}$ ergibt. Dies ist sehr viel empfindlicher als es piezoelektrische Aktuatoren mit etwa $0.3 \mu \mathrm{m} / \mathrm{kV}$ sind (Séchaud 1999).

Die statische Zustandsgleichung eines idealen bimorphen Spiegels hat nach Roddier (1988) die Form

$$
\nabla^{2}\left(\nabla^{2} \varphi+A V\right)=0,
$$

wobei $\nabla^{2}$ den zweidimensionalen Laplace-Operator darstellt, $\varphi(x, y)$ die Spiegeloberfläche, $V(x, y)$ die Spannungsverteilung auf der Scheibe und $A=8 d_{31} / t^{2}$.

Wichtig zu erwähnen ist an dieser Stelle, dass der Gleichgewichtszustand erreicht ist, wenn die Spiegeloberfläche die Lösung einer Poisson-Gleichung mit passenden Randbedingungen darstellt. Letztere werden durch radiale Neigungen am Rand des Spiegels geboten. Eine einfache Lösung zur Kontrolle dieser Verkippungen bietet die Verwendung eines größeren Spiegels mit einem zusätzlichen äußeren Elektrodenring, während das Bild der Teleskoppupille auf den inneren Bereich des Spiegels begrenzt bleibt.

Zum Versatz in z-Richtung trägt neben der Biegung der Oberfläche auch die Änderung der Dicke des Spiegels bei. Von Gleichung 4.18 leitet man eine Änderung der Auslenkung

$$
\varphi_{t}(x, y)=-b V(x, y) d_{31}
$$

über einer Elektrode durch Dickenänderung ab, die mit der Auslenkung $\varphi_{b}$ durch reine Verbiegung verglichen werden muss und durch Gleichung 4.17beschrieben ist. So dominiert der Krümmungseffekt, wenn die Spannung über eine Fläche anliegt, deren lineare Abmessungen $d$ groß gegenüber der Dicke $t$ ist. Auf kleiner Skala $(d<4 t)$ ist die Dickenänderung der größere Effekt und begrenzt die korrigierbaren Raumfrequenzen nach oben. Daraus ergibt sich eine Obergrenze für die Anzahl der Elektroden, die durch das Verhältnis von Durchmesser zur Dicke des bimorphen Spiegels bestimmt wird. Typischerweise werden einige zehn Elektroden benützt, d. h. diese Spiegelsorte ist am besten für Systeme geeignet, die nur Moden relativ niedriger Ordnung korrigieren ('low order compensation'). Einen wichtigen Einfluss auf die Anzahl der korrigierbaren Moden hat jedoch die Wahl der Geometrie für die Verteilung der Elektroden. Wie Scharmer et al. (2003b) beschrieben haben, eignet sich die sog. Keystone-Geometrie hervorragend zur Nachbildung azimuthaler Sinus, wie sie bei Zernike- und KL-Moden vorkommen. 



\section{AO-Simulation}

\section{1 Überblick}

Atmosphärisch verursachte Distorsionen von ursprünglich ebenen Wellenfronten haben aufgrund ihres turbulenten Ursprunges einen nur im statistischen Mittel abschätzbaren Einfluss auf die Abbildungsqualität erdgebundener Teleskope. Die momentane Deformation der WF ist jedoch nicht bekannt und auch mit den Messwerten eines Wellenfrontsensors nur im Nachhinein, und nur mit Einschränkungen und bis zu einer gewissen Genauigkeit bestimmbar. Daraus erwächst der Bedarf an Simulationen solcher Wellenfronten und den zugehörigen Punktverbreiterungsfunktionen. Gerade in der Planungsphase von Adaptiven Optiken dienen Simulationen der Bestimmung optimaler Systemparameter und zur Abschätzung der effektiven optischen Qualität, da sich alle relevanten Parameter konkret errechnen lassen. Um im wissenschaftlichen Betrieb höchste räumliche Auflösung des Beobachtungsobjektes zu erreichen, sind oft Nachbehandlungen der Daten notwendig, die auf der Entfaltung mit einer geeigneten PSF beruhen oder einen solchen Schritt beinhalten, wie etwa die Speckle-Bildrekonstruktion. In diesen Fällen sind alle Messwerte und alle bekannten Eigenschaften des Systems, die Hinweise auf optische Fehler während der Datengewinnung enthalten, wichtig und geeignet auszuwerten, damit beste Resultate gewonnen werden können.

\subsection{Generierung von Phasenschirmen mit dem Programm- paket TurbuLenZ}

Der TuRbuLenZ-Code $\mathrm{e}^{1}$ stammt ursprünglich von einem C-Programm zur Simulation der zeitlichen Entwicklung von Speckle-Mustern ab (Glindemann et al.|1993, Lane et al. 1992), das von Berkefeld (1998) erweitert und später von Weiß (2003) optimiert und in die Programmiersprache IDL übersetzt wurde. Das Programmpaket ist in der Lage, die optischen Einflüsse, also Phasenretardation und Szintillation, einer durch Turbulenzschichten modellierten Atmophäre zu simulieren und wird durch eine Parameterdatei konfiguriert, die alle benötigten Informationen enthält. Im Rahmen dieser Arbeit wird TURBULENZ dazu verwendet, Phasenschirme ('phase screens', PHS) zu erzeugen, die die Phasenverzögerungen einer einfallenden, ursprünglich ebenen WF auf einem gewissen zweidimensionalen Ausschnitt darstellen, nachdem die WF durch die Turbulenzschichten getreten ist. Die PHS werden im Fourierraum gemäß einem Komogorov'schen Potenzgesetz durch

\footnotetext{
${ }^{1}$ Der Code in Version 1.0 ist vom MPIA in Heidelberg frei zu beziehen: http://www.mpia-hd.mpg.de/AO/ATMOSPHERE/TurbuLenZ/tlz.html
} 
spektrale Synthese berechnet und dienen dann als Eingabegrößen für den eigentlichen AO-Simulationscode. Gelegentlich wurde von der Möglichkeit Gebrauch gemacht, auch die Intensitätsfluktuationen an der Eintrittspupille des Teleskops (Amplitudenschirme) in die Berechnungen der Transferfunktionen miteinzubeziehen. Da starke Szintillation nichtlineares Verhalten zeigt, das in TURBULENZ nicht modelliert wird, kann hier immer nur von schwacher Szintillation $(\delta I<0.1 I)$ ausgegangen werden. Diese hat aber auf die Korrelationsmessungen mit SHS-Bildern der Photosphäre der Sonne kaum messbaren Einfluss und hat hier keine vorrangige Bedeutung.

\subsubsection{Eingabe-Parameter}

Die Parameterdatei bietet dem Nutzer die Möglichkeit zur Kontrolle wichtiger Parameter, welche die gewünschte Beobachtungssituation modellieren, also Kenngrößen der Turbulenz in den angenommenen Schichten der Erdatmosphäre, geometrische Maße des Beobachtungsinstruments und die Größe des Bildfeldes. Die ersten vier Punkte betreffen alle erzeugten Phasenschirme gleichermaßen. Die restlichen Punkte 5-7 geben Anlass zur Erzeugung mehrerer Sets von PHS, jeweils für eine Kombination der Parameter.

1. Anzahl der Zeitschritte bzw. zu erzeugenden Phasenschirme

2. Beobachtungswellenlänge in Metern

3. Mindesthöhendifferenz für Fresnel-Propagation in Metern

4. Pixelskala in der Aperturebene in Pixel/Meter

5. Anzahl der Teleskope, jeweils mit:

(a) Durchmesser der Blendenöffnung in Metern

(b) Relative Positionskoordinaten (x,y) in Metern

(c) Bildgröße in der Fokalebene in Pixeln

(d) Pixelskala des Detektors in Bogensekunden pro Pixel

6. Anzahl der Sichtlinien, jeweils mit relativem Winkelabstand entlang der Achsen $(\mathrm{x}, \mathrm{y})$ in Bogensekunden

7. Anzahl der Turbulenzschichten, jeweils unter Angabe von:

(a) Höhe der Schicht über dem Teleskop in Metern

(b) 'Lokaler' Fried-Parameter $r_{0}$ in Metern

(c) 'Outer scale' der lokalen Turbulenz in Metern

(d) Dekorrelationsparameter (nach G1. 5.6)

(e) Windgeschwindigkeit in Meter pro Zeitschritt und Windrichtung in Grad

Aus den Angaben werden zunächst die benötigten Abmessungen der PHS jeder Turbulenzschicht in Pixeln berechnet und diese PHS für jeden Zeitschritt erzeugt, wie im folgenden Abschnitt 5.2.2 näher erklärt wird. Einige Bemerkungen zu dynamisch wichtigen Größen folgen danach im Abschnitt 5.2.3 unter dem Aspekt der zeitlichen Entwicklung der Turbulenz. 


\subsubsection{Berechnung der Phasenschirme}

Ausgangspunkt für die Berechnung der Phase $\varphi(\boldsymbol{x})$ ist das Kolmogorov-Powerspektrum $W_{\varphi}(\boldsymbol{k})=0.023 r_{0}^{-5 / 3} k^{-11 / 3}$. Der Betrag $|\hat{\varphi}(\boldsymbol{k})|$ der Fouriertransformierten der Phase ist gegeben durch die Wurzel des Powerspektrums, und wird mit den Koordinaten $(k, l)$ im Fourierraum numerisch durch

$$
\hat{\varphi}(k, l)=\sqrt{0.023}\left(\frac{D N_{\text {pix }}}{D_{\text {pix }} r_{0}}\right)^{5 / 6}|(k, l)|^{-11 / 6} e^{i \Phi(k, l)}
$$

dargestellt. Hier bezeichnet $D$ den Durchmesser der Apertur in Meter und $N_{p i x}$ ist die Größe des Phasenschirms in Pixeln, die etwa das Dreifache des Aperturdurchmessers $D_{p i x}$ in Pixeln betragen sollte, um Randeffekte durch das Sampling zu vermeiden. Die mittlere Phase, also der Gleichanteil $\hat{\varphi}(0,0)$ wird dabei auf Null gesetzt. Die zufälligen Fluktuationen werden einerseits dadurch erzeugt, dass mit einer Gaußverteilten Zufallszahl der Varianz Eins multipliziert und eine gleichverteilte Phase $\Phi$ hinzuaddiert wird. Dadurch wird gesichert, dass die Durchschnittswerte vieler solcher Realisationen wieder das -11/3-Potenzgesetz zeigen. Die Rücktransformation des nun vollständigen Spektrums liefert den Phasenschirm $\varphi(\boldsymbol{x})$ in Einheiten Radian.

Bedingt durch die endliche Größe $N_{\text {pix }}^{2}$ (in Pixeln) dieser Phasenschirme wird allerdings das Spektrum bei niedrigen Raumfrequenzen nicht gut modelliert und weist somit effektiv eine 'outer scale' (siehe 3.2.4) von der Größe der verwendeten Phasenmaske auf, während die tatsächlichen Werte bis zur Größenordnung 100 m reichen. Für Entfernungen zwischen auseinanderliegenden Turbulenzgebieten, die größer als der Teleskopdurchmesser sind, wirken die zugehörigen tiefen Frequenzen der Korrelationen aber als zusätzlicher Tilt-Anteil, der in der Simulation so nicht erzeugt werden kann. Die resultierende Gesamtneigung der Wellenfront wird also nur unbefriedigend nachgebildet. Die Änderungen des Spektrums nahe dem Ursprung im Fourierraum sind zu groß, als dass der einzelne Abtastpunkt der diskreten Fouriertransformation ausreichte, das Fourierintegral zu modellieren. Dieser Effekt wurde jedoch berücksichtigt und durch die Addition von sog. Subharmonischen nach Lane et al. (1992) abgemildert, d. h. Frequenzen, denen Periodenlängen entsprechen, die größer als das Datenfeld sind. Die Idee besteht dabei darin, in einem ersten Schritt $(i=1)$ das Pixel am Ursprung durch ein Feld von $3 \times 3$ Subpixeln zu ersetzen, die den kritischen Bereich feiner abzutasten vermögen. Die gleiche Vorgehensweise kann nun so oft $(i=2,3, \ldots)$ mit dem jeweils am Ursprung liegenden Subpixel wiederholt werden, bis eine Frequenz $f=\left(2 N_{\text {pix }} \cdot 3^{i}\right)^{-1}$ erreicht wird, die der gewünschten 'outer scale' entspricht. Entsprechend ihrer kleineren Fläche muss jedoch jedes Sample mit einem Gewichtungsfaktor $G(i)=1 / 9^{i}$ in die Näherung genommen werden. Es ergibt sich ein Phasenspektrum der subharmonischen Frequenzen von

$$
\begin{aligned}
\hat{\varphi}_{\text {sub }}(k, l)=\sum_{i} \sqrt{0.023}\left(\frac{D N_{\text {pix }}}{D_{\text {pix }} r_{0}}\right)^{5 / 6} & \operatorname{sinc}\left[\pi\left(k-k_{s}(i)\right)\right] \operatorname{sinc}\left[\pi\left(l-l_{s}(i)\right)\right] \\
& \times G(i)\left|k_{s}(i), l_{s}(i)\right|^{-11 / 6} e^{i \Phi\left(k_{s}, l_{s}\right)}
\end{aligned}
$$

mit den subharmonischen Frequenzen $l_{s}(i)$ und $k_{s}(i)$. Das endgültige Gesamtspektrum ergibt sich dann zu

$$
\hat{\varphi}_{\text {gesamt }}(k, l)=\hat{\varphi}(k, l)+\hat{\varphi}_{\text {sub }}(k, l) \text {. }
$$


Das zentrale Pixel des Phasenschirms wird endlich auf den Mittelwert seiner umgebenden Pixel gesetzt, weil hier durch die Einführung der subharmonischen Frequenzen häufig deutlich größere Werte als in der Umgebung auftreten, was im Weiteren zu Fehlern führt.

\subsubsection{Modellierung der Dynamik}

Zur Simulation dynamischer Entwicklung müssen zwei Punkte umgesetzt werden. Zum Einen Wind, der die Turbulenzzellen quer zur Lichtausbreitungsrichtung mit sich trägt, zum Anderen die innere Entwicklung der Turbulenz, die sich in statistischen Fluktuationen von einem Zeitschritt zum nächsten zeigen, sodass sich das Muster der Phasenverzögerungen mehr oder weniger langsam verändert.

\subsubsection{Wind}

Zur Modellierung des Windes besteht zunächst die Möglichkeit, einen sehr großen PHS zu erzeugen und ihn förmlich vor der Teleskoppupille vorbeiziehen zu lassen. Dies entspricht der Taylor'schen 'frozen flow'-Hypothese und kann als erste Näherung, insbesondere bei großen Windgeschwindigkeiten, benutzt werden. Im Code wird diese Verschiebung des PHS von einer Realisation zur Nächsten durch die Addition einer linearen Funktion zur Phase des komplexen Spektrums umgesetzt, bevor der für das Teleskop sichtbare Bereich aus dem PHS ausgeschnitten wird:

$$
\hat{\varphi}_{\text {shift }}(k, l)=\hat{\varphi}_{\text {alt }}(k, l) \exp \left(2 \pi i k N_{s} / N_{\text {pix }}\right),
$$

wobei $N_{s}$ die Anzahl der Pixel ist, um die der PHS in Richtung der Frequenzkoordinate $k$ verschoben werden soll, und $N_{\text {pix }}$ wieder die lineare Abmessung des PHS in Pixeln bezeichnet. Dabei ist $N_{s}$ nicht auf ganzzahlige Werte beschränkt, da die Operation im Fourierraum $(k, l)$ ausgeführt wird.

Allerdings sei darauf hingewiesen, dass die Wind-Parameter im Eingabefile (siehe Punkt 7e in Abschnitt 5.2.1) nur in Verbindung mit einem ausreichend niedrigen Dekorrelationsparameter (Gl. 5.6) sinnvoll ist, d. h. wenn die Phasenschirme so stark von einander abhängen, dass sie eine scheinbar kontinuierliche zeitliche Entwicklung der Turbulenz erkennen lassen. Die Verschiebung des PHS zur Simulation von Wind nach G1. 5.4 verliert bei instantanen, unabhängigen Phasenschirmen ihren Sinn. Sind jedoch in diesem Fall die Phasenverzögerungen vor und nach einer kleinen Zeitverzögerung von Interesse, wie z. B. die im Falle der Messung und der Korrektur einer Wellenfront durch AO, kann im Eingabe-File einem eventuellen Wind auch mit der Angabe eines weiteren Teleskops (Punkt 5) Rechnung getragen werden, dessen Lage entsprechend der Windgeschwindigkeit und -richtung gemäß dem angegebenen Zeitschritt von Teleskop 1 entfernt definiert wird. Der vom zweiten Teleskop gleichzeitig 'gesehene' Ausschnitt entspricht dann einem zeitlich versetzten PHS des ersten Teleskops. Diese Vorgehensweise ist im Falle der Simulation von unabhängigen Speckle-Bildern effektiver, da für beliebig viele verschiedene Windbedingungen nur ein PHS erzeugt werden muss.

\subsubsection{Dekorrelation}

Zieht das Phasenmuster langsam über das Teleskop, kann jedoch die Statistik eines SpeckleMusters ('speckle boiling') auf der nötigen Zeitskala nicht mehr realistisch modelliert 
werden. Nötig wird also hier das Einbeziehen der inneren Entwicklung der Turbulenz bzw. des Phasenschirmmusters. Zur Modellierung werden im TURBULENZ-Code die zugehörigen Zufallszahlen im Fourierspektrum $\hat{\varphi}_{\text {old }}$ des PHS $\varphi_{\text {old }}$ mit einem MarkovProzess justierbarer Stärke verändert (Glindemann et al. 1993). Dazu wird ein zweites Zufallszahlenfeld $\hat{\varphi}_{\text {tmp }}$ erzeugt und mit dem ersten Spektrum nach der Anweisung

$$
\hat{\varphi}_{\text {neu }}(k, l)=\frac{\hat{\varphi}_{\text {alt }}(k, l)+\alpha(k, l) \hat{\varphi}_{\text {tmp }}(k, l)}{\left[1+(\alpha(k, l))^{2}\right]^{1 / 2}}
$$

zum Ergebnisspektrum verbunden. Der Dekorrelationsparameter

$$
\alpha(k, l)=t|(k, l)| \frac{20}{N_{\text {pix }}}
$$

mit der linearen Ausdehnung $N_{\text {pix }}^{2}$ der PHS-Matrix in Pixeln und der Zeit $t$ zwischen zwei PHS-Realisationen in Millisekunden, steuert die Geschwindigkeit des Entwicklungsprozesses. $\alpha$ ist proportional zum Betrag $|(k, l)|$ der Raumfrequenzen gesetzt, sodass die zu hohen räumlichen Frequenzen gehörenden kleinen Strukturen schneller dekorrelieren als große Strukturen, was physikalisch Sinn ergibt. Diese Prozedur wird für jede neue Realisation eines PHS wiederholt, sodass jeder simulierte Zustand nur vom vorigen, älteren Zustand abhängt, aber nicht von wiederum dessen Vorgängern. Diese Eigenschaft macht aber genau einen Markov-Prozess aus.

Die Kombination des Markov-Prozesses mit dem bewegten Phasenschirm gelingt durch Einsetzen von $\hat{\varphi}_{\text {shift }}$ aus Gl. 5.4 an Stelle von $\hat{\varphi}_{\text {alt }}$ in Gl. 5.5. Über die Angabe des PHSVersatzes $N_{s}$ (Windgeschwindigkeit) und des Dekorrelationsparameters $\alpha$ (zeitlicher Abstand zwischen zwei PHS) ist es also möglich, der Statistik der dynamischen Entwicklung auf langen und kurzen Zeitskalen Rechnung zu tragen.

\subsubsection{Wellenfrontpropagation}

Die Wellenfrontpropagation durch die Modellatmosphäre erfolgt für jedes hypothetische Teleskop jeweils für alle Blickrichtungen gesondert. Im Allgemeinen führt nämlich jede Kombination dieser Größen zu anderen Positionen im PHS, an der der vom Teleskop aufgefangene Lichtzylinder durch die jeweiligen Turbulenzschichten tritt. Nur der durch die Schnittfläche definierte Bereich der PHS um diese Positionen wird bei der Propagation berücksichtigt. Wenn die Höhendifferenz zwischen zwei aufeinanderfolgenden Schichten kleiner als die in der Parameterdatei (siehe Abschnitt 5.2.1) unter Punkt 3 gewählte ist, werden die PHS-Ausschnitte einfach addiert, ohne dass sich die Amplitude ändert. Physikalisch ist dies sinnvoll, da sich Phasenverzögerungen tatsächlich aufsummieren und die Distanz für die Selbstinterferenz der WF (Szintillation) noch als zu klein angenommen wird. Erst, wenn die Höhendifferenz $z_{d}$ der Bedingung

$$
z_{d}^{3} \gg \frac{\pi}{\lambda} D^{4}
$$

genügt, sollte die Fresnelnäherung benutzt werden (vgl. Abschnitt 2.2.1). Für das VTT gilt bei sichtbaren Wellenlängen $z_{d} \gg 200 \mathrm{~m}$, was für diese Arbeit keine praktischen Probleme bereitet, da hier neben der bodennahen Turbulenz nur die Grenzschichtturbulenz in der Tropopause angenommen wird. 

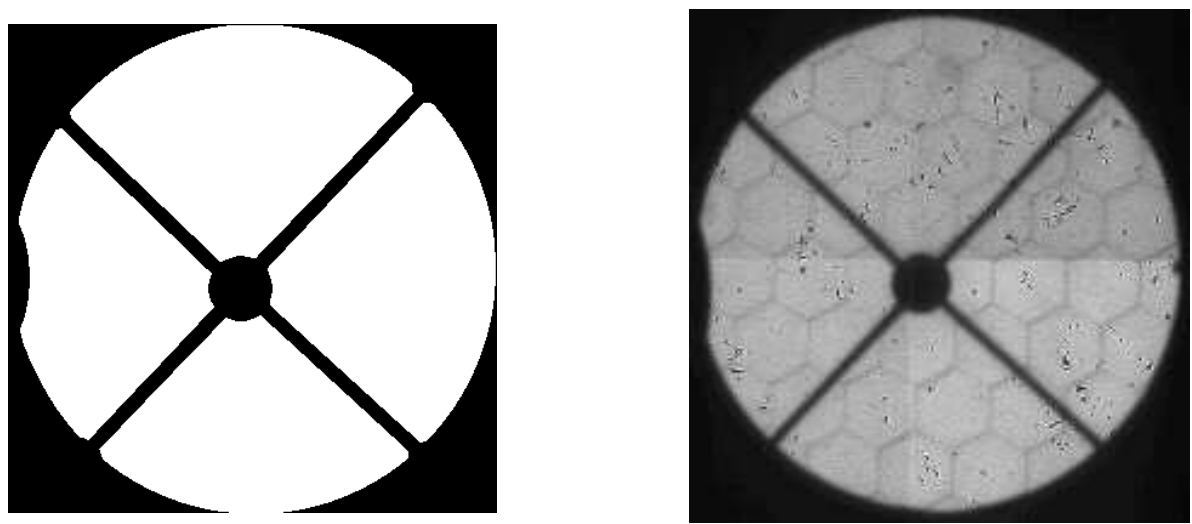

Abbildung 5.1: Die Eintrittspupille des VTT. Links: Aus dem rechten Bild abgeleitete Maske zur Darstellung der Pupillentransmissionsfunktion im Simulationscode. Rechts: Tatsächliche Aufnahme mit der Kamera in einer konjugierten Ebene zur Pupillenebene. Neben den Abschattungen durch den Spiegel zur Nachführung und seiner Halterung ist im Hintergrund die Anordnung der Subaperturen des WFS zu erkennen.

\subsection{Einfache Modellierung von KAOS}

Ziel der Simulationen ist unter Anderem die Charakterisierung der Übertragungseigenschaften des zur Datengewinnung verwendeten Teleskops und seinem AO-System. Beobachtet wurde im Rahmen dieser Arbeit mit dem deutschen Sonnenteleskop VTT (VakuumTurm-Teleskop) und seinem AO-System KAOS (Kiepenheuer Adaptive Optics System) und dem schwedischen SST (Swedish 1-m Solar Telescope). Also gilt es, wichtige optische Eigenschaften dieser Teleskope nachzubilden und in das Simulationsprogramm zu implementieren. Die wichtigsten geometrischen Größen in diesem Zusammenhang bilden Teleskopöffnung, Anordnung der Subaperturen des WFS und die Verteilung der Elektroden des bimorphen Spiegels über seine Fläche. Bei der Eintrittspupille müssen ihre Form und evtl. Obstruktionen, wie im Falle des VTT der Sekundärspiegel zusammen mit seinem Haltekreuz, berücksichtigt werden. Die Abbildung dieser Pupillentransmissionsfunktion auf eine Matrix liefert bei entsprechender Pixelskala eine Maske, mit der die jeweiligen Phasenschirme multipliziert werden. Abbildung 5.1 zeigt eine tatsächliche Aufnahme der VTT-Eintrittspupille, und der daraus gebildeten Maske, die im Durchlassbereich mit einer Transmission von $100 \%$ modelliert wurde. Für das SST wurde lediglich eine kreisförmige Apertur ohne weitere Abdeckungen angenommen.

Das Lenslet-Array des WFS liegt in einer optisch zur Eintrittspupille konjugierten Ebene, d.h. Strukturen am tatsächlichen Ort der Pupille, wie etwa die Haltevorrichtung eines Spiegels für die Nachführung, werden (verkleinert) dorthin abgebildet. Dies gilt insbesondere für die aberrationenbehaftete Wellenfront selbst. Der kleine Strahlquerschnitt wird nun von der flächenfüllenden Anordnung der kleinen Linsen so überdeckt, dass die Subaperturen möglichst gut ausgeleuchtet werden, wie in Abb. 5.2 angedeutet ist. Für die Abschattung durch den Nachführspiegel ergibt sich als beste Lösung die vollständige Bedeckung einer einzigen Subapertur, im Gegensatz zum Hintergrundmuster des linken Bildes von Abb. 5.1, wo zwei Hexagone jeweils zur Hälfte bedeckt werden. Der Abbildungsmaßstab wurde so gewählt, dass ein Durchmesser des Strahlbündels durch sieben 
Pupillenebene

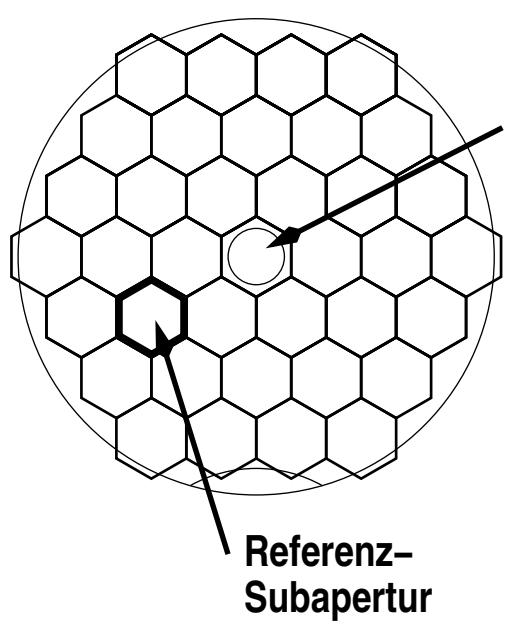

\section{Fokalebene}
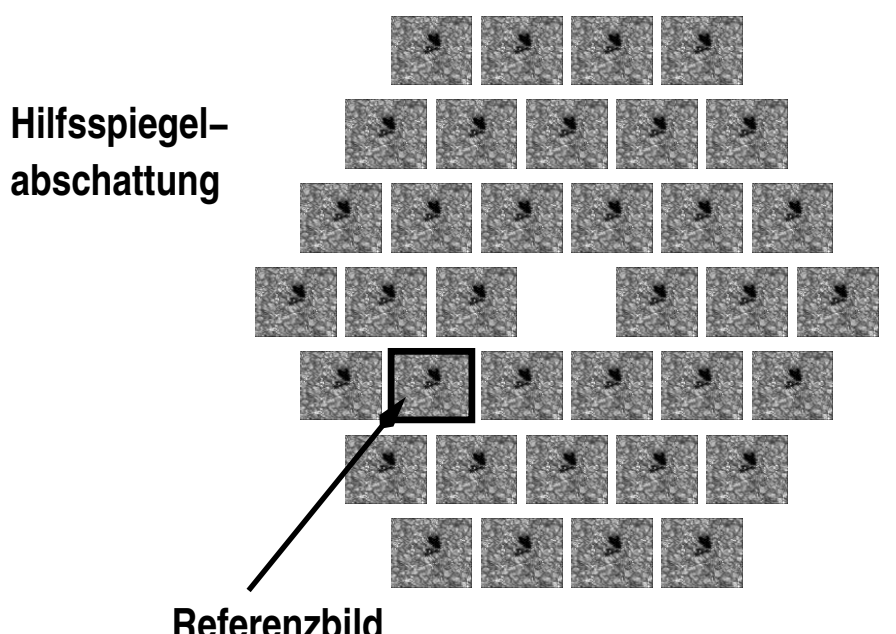

Abbildung 5.2: Zur Geometrie des KAOS-Wellenfrontsensors. Links: Das Bild der Pupille wird durch das Lenslet-Array des SHS überdeckt. Die zentrale Subapertur wird durch den Sekundärspiegel abgeschattet. Rechts: Jede Linse des Lenslet-Arrays formt ein Bild des gleichen Objektausschnitts am CCD des Sensors. Durch Kreuzkorrelation mit dem Referenzbild wird für jede Subapertur die Wellenfrontneigung ermittelt.

nebeneinanderliegende Linsen überdeckt werden kann. Da der Durchmesser des VTT $0.7 \mathrm{~m}$ beträgt, entspricht den einzelnen Lenslets, in die Eintrittspupille des Teleskops zurückprojiziert, ein Bereich vom Durchmesser $10 \mathrm{~cm}$. Ein Fried-Parameter dieser Größe beschreibt recht ordentliche Seeing-Bedingungen, bei denen für den möglichen Korrekturgrad eine sehr hohe Strehl-Zahl bei noch guter Effizienz erreicht werden kann.

\subsubsection{Wellenfrontmessung}

Zur Messung der Wellenfront wird die mittlere Neigung der Wellenfront über jeder Subapertur des Lenslet-Arrays bestimmt. Da wir in der Simulation die tatsächliche WF vorliegen haben, muss hier nicht der 'Umweg' über die Kreuzkorrelation der Subfelder des WFS nachmodelliert werden. Stattdessen wird die durchschnittliche Verkippung entlang der beiden Koordinatenachsen direkt bestimmt: Dazu wurde folgendermaßen vorgegangen: Eine simulierte Wellenfront wird maßstabsgetreu auf das hexagonale Lenslet-Array projiziert und für jede Subapertur $s$ der entsprechende Ausschnitt $A_{s}$ der WF aus der Datenmatrix ausgeschnitten. Wie bei etwa einer Zernike-Modenzerlegung sucht man für jede Subapertur gewissermaßen die 'Tip/Tilt'-Koeffizienten $a_{2,3}$ (Noll'sche Zählung), indem man $A_{s}$ mit einem ihm entsprechenden Ausschnitt $T_{s}$ einer ebenen, aber in x-bzw. y-Richtung geneigten Fläche multipliziert. Wenn letztere im Sinne einer Modenentwicklung jeweils orthogonal zu allen anderen Deformationen sind, bleibt beim Integral über das Produkt $T_{s} A_{s}$ nur der Beitrag der Mode $T_{2}$ bzw. $T_{3}$ und der Wert entspricht dem Entwicklungskoeffizienten $a_{2}$ bzw. $a_{3}$ dieser Mode, multipliziert mit der Varianz der Modenauslenkung selbst. Der Kehrwert der Varianz ist somit auch gleich dem Normierungs- 

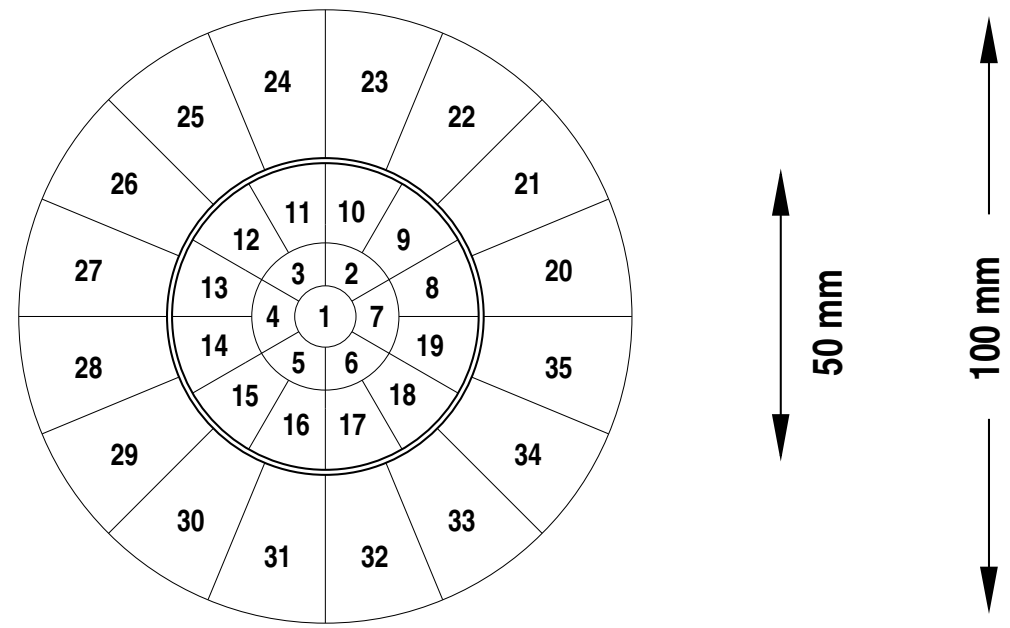

Abbildung 5.3: Verteilung der 35 Aktuator-Elektroden des deformierbaren Spiegels (DM) von KAOS. Der Spiegel ist vom Typ Bimorph. Nur der zentrale Bereich mit einem Durchmesser von $50 \mathrm{~mm}$ wird ausgeleuchtet.

faktor. Bei eventuellen Abschattungen der Subapertur, z. B. durch die Halterung des Sekundärspiegels, ist auf das richtige Integratisonsgebiet zu achten, das mit dem tatsächlichen Durchlassbereich für die jeweilige Subapertur identisch ist. Als Produkt der bekannten Neigung von $T_{s}$ und dem berechneten Koeffizienten wird diejenige Verkippung der WF im Ausschnitt $A_{s}$ berechnet, die bei Korrektur den rms-Wert des WF-Fehlers minimiert. Um später gemessene und simulierte Werte miteinander vergleichen zu können, müssen die WF-Neigungen noch in einen Bildversatz umgerechnet werden. Dazu genügt eine strahlenoptische Rechnung, für die wir annehmen können, dass für jedes Lenslet der Hauptstrahl mit der optischen Achse den gleichen (kleinen!) Winkel $\boldsymbol{\alpha}=\left(\alpha_{x}, \alpha_{y}\right)$ bildet wie die gemessene Neigung der WF innerhalb einer Subapertur. Weiter entspricht der Eintrittswinkel des Hauptstrahls dem bildseitigen Austrittswinkel, da er durch die Linse nicht abgelenkt wird. Um nun auf den lateralen Versatz $\Delta x$ in der Fokalebene zu kommen, muss nach Gl. 4.1 die Brennweite $f$ der Linsen des WFS und deren Vergrößerungsfaktor $M$ im Vergleich zur Teleskoppupille bekannt sein. Alternativ kann das Produkt $f M$ durch eine effektive Brennweite $f_{\text {eff }}$ ersetzt werden, die sich aus der Pixelgröße des WFS-CCD in Verbindung mit dem Bildmaßstab ergibt:

$$
f_{\text {eff }}=\frac{\text { Pixelgröße }}{\text { Pixelskala }}=\frac{12 \mu \mathrm{m}}{0.5^{\prime \prime}}=4.95 \mathrm{~m} .
$$

Dabei haben wir $\tan \alpha \approx \alpha$ benutzt.

\subsubsection{Deformierbarer Spiegel}

KAOS besitzt einen bimorphen DM der Firma Laplacian mit 35 Flächenelektroden, die nach einer 'Keystone-Symmetrie' angeordnet sind (Abb. 5.3). Dies wurde bei der Modellierung der Deformationseigenschaften des Spiegels für die Simulation folgendermaßen berücksichtigt: An jeder Elektrode wird jeweils eine gewisse 'Standard-Spannung' angelegt, d. h. die Spannungsverteilung $V(x, y)$ über der Spiegelfläche ist in diesem Bereich 

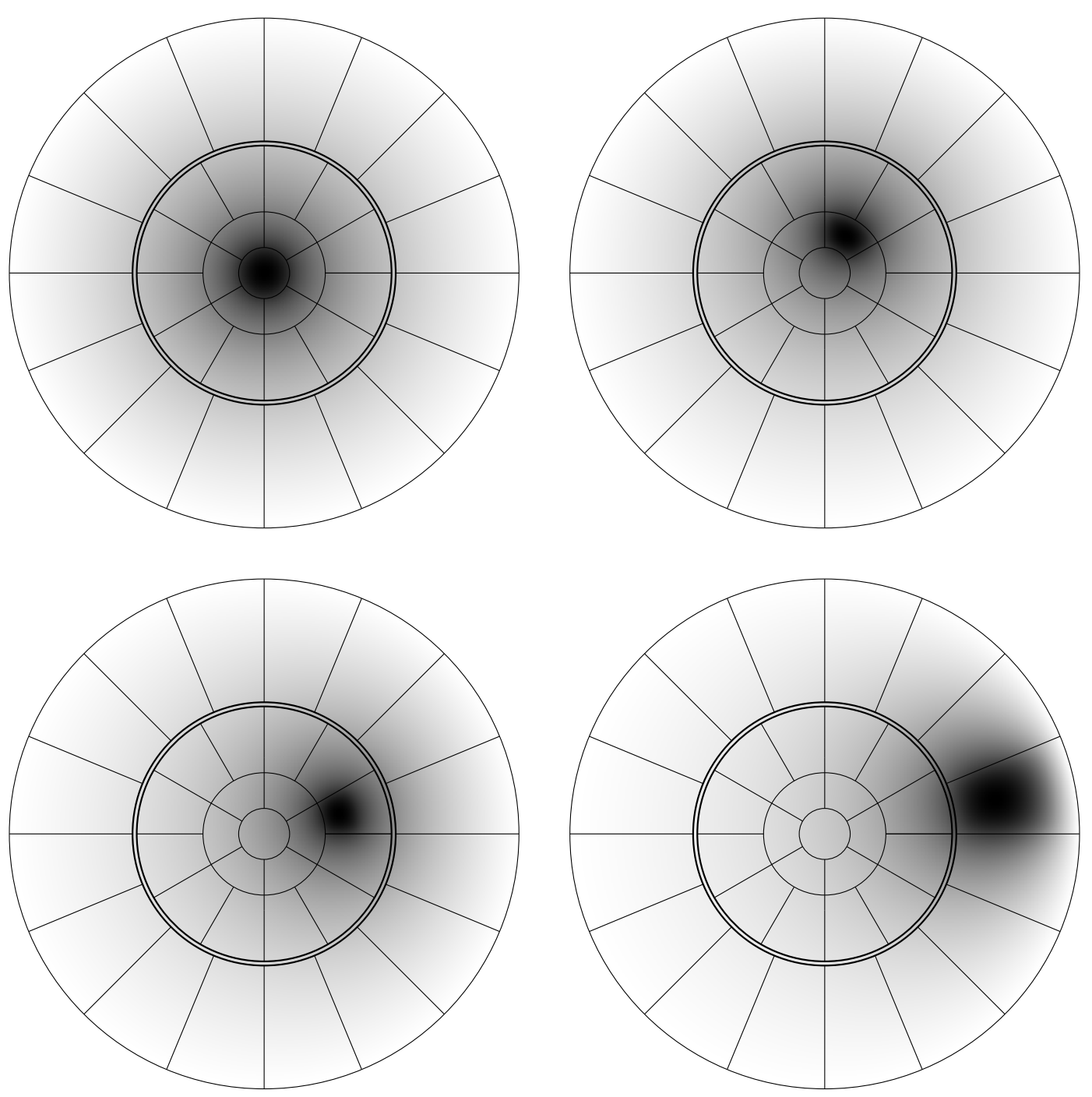

Abbildung 5.4: Die Einflussfunktionen des DM für jeden Ring von Aktuatoren (vgl. Abb. 5.3 beschreiben die Auslenkung des Spiegels aus der Ebene, hier durch Grauwerte kodiert. Sie wird durch Anlegen einer Spannung an eine einzelne Elektrode des Spiegels verursacht. Für jeden Ring muss nur eine Funktion berechnet werden, die übrigen können durch Drehung in der Ebene daraus erzeugt werden.

Eins, während die übrige Fläche neutral angenommen wird. Mit dieser Anfangsbedingung wird in einem iterativen Prozess die Lösung der Poisson-Gleichung zweiten Grades (Gl. 4.18) für die Spiegeloberfläche angenähert, bis eine genügend glatte Auslenkungsfunktion über den gesamten Spiegel erreicht ist. Für jeden Ring von Aktuatoren ist jeweils nur eine dieser sogenannten Einflussfunktionen zu berechnen, da sie durch Rotation auseinander hervorgehen. In Abbildung 5.4 sind die vier benötigten Einflussfunktionen dargestellt. Für die Berechnung der Interaktionsmatrix (Abschnitt 5.3.3) müssen sie allerdings für jede Aktuatorposition als Datenmatritzen vorliegen. 


\subsubsection{Kalibration}

Im laufenden Betrieb sollen bei geschlossenem Regelkreis ('closed loop') aus den WFSMessungen passende Signale für den DM errechnet werden, die eine kontrollierte Spiegeldeformation zur Korrektur der gemessenen WF bewirken. Dazu muss der Effekt eines DM-Signals und der dadurch verursachten Spiegelverformung auf die Wellenfront quantitativ bekannt sein. Deshalb müssen WFS und DM gegeneinander kalibriert, also die Interaktion zwischen beiden festgehalten werden.

Bei der Kalibration wird zunächst eine Lochblende ('Pinhole') im Fokus des Teleskops positioniert, sodass sich nach dem Kollimationsspiegel Licht in parallelem Strahlengang bzw. als ebene WF ausbreitet. Als solches trifft es in der konjugierten Ebene zur Pupille auf den DM und später das Lenslet-Array des WFS, wo jede Linse das Pinhole auf das WFS-CCD abbildet. Bei neutralen Aktuator-Elektroden, also flachem DM, ergibt sich dann in der Fokalebene des WFS nach der Anordnung des Lenslet-Arrays ein regelmäßiges Gitter aus Pinhole-Bildern (vgl. Abb. 4.3). Nacheinander wird nun jede Elektrode angesteuert (vgl. Abb. 5.4) und mit einer definierten Standard-Spannung geladen und prägt der ebenen WF nach dem Prinzip der Phasenkonjugation (siehe Abb. 4.4) eine Deformation auf, die je nach ihrer lokalen Neigung eine gewisse charakteristische Verzerrung des vorher regelmäßigen Musters der $N_{\text {subap }}$ Pinhole-Bilder bewirkt. Die gemessenen Verschiebungen entlang der Koordinatenachsen werden als Datenvektor der Länge $2 N_{\text {subap }}$ für jeden Aktuator gespeichert. Alle Messungen zusammen ergeben dann die Interaktionsmatrix.

\subsubsection{Singular Value Decomposition}

Um nun umgekehrt von den WFS-Daten auf die WF-Deformation schließen zu können, muss die Matrix invertiert werden, was meist nur annähernd möglich ist und beispielsweise durch die Methode der kleinsten Fehlerquadrate erreicht wird. Wie bei KAOS wird dazu eine Singulärwertzerlegung vorgenommen ('singular value decomposition', SVD) um zu einer Pseudo-Inversen der Matrix zu gelangen. Die SVD ermöglicht es, jede komplexe Matrix $A \in \mathbb{C}^{m \times n}$ in drei Matrizen

$$
A=U S V^{*}
$$

zu zerlegen (vgl. Abb. 5.5), wobei $U \in \mathbb{C}^{m \times m}$ wie auch $V \in \mathbb{C}^{n \times n}$ orthonormale Spaltenvektoren besitzen, sodass gilt

$$
\begin{aligned}
& U U^{*}=U^{*} U=E_{m} \quad \text { bzw. } \\
& V V^{*}=V^{*} V=E_{n},
\end{aligned}
$$

d.h. das Produkt der Matrizen mit ihren Adjungierten ergibt die jeweilige Einheitsmatrix. Hat die Matrix $A$ den Rang $r$, so weist die Matrix $S \in \mathbb{C}^{m \times n}$ lediglich $r$ positive, der Größe nach geordnete Diagonalelemente auf, die sogenannten Singulärwerte von $A$. Eventuelle weitere Diagonalelemente sind gleich Null. Die Singulärwerte charakterisie- 


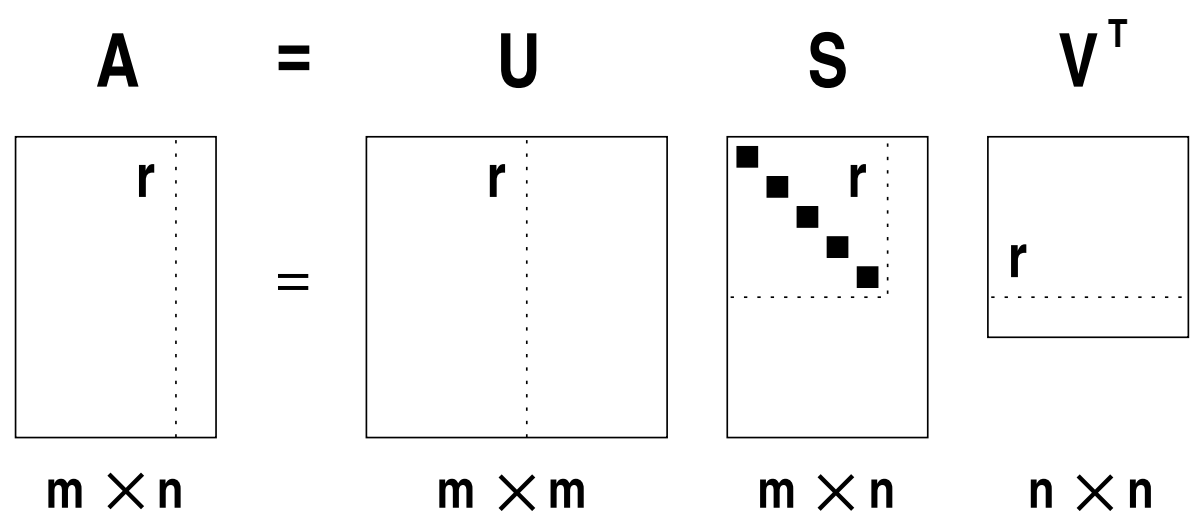

Abbildung 5.5: Veranschaulichung zur Singulärwertzerlegung (SVD). Die Spaltenvektoren von $U$ sowie von $V$ sind orthonormal, Matrix $S$ besitzt von Null verschiedene Einträge lediglich auf der Diagonale. Die Anzahl $\mathrm{r} \leq \min (m, n)$ der Singulärwerte in Matrix $S$ entspricht dem Rang der Matrix $A$. SVD ermöglicht eine einfache, jedoch verlustbehaftete Berechnung der (Pseudo-)Inversen der Matrix $A$.

ren allgemein eine (m,n)-Matrix ähnlich wie Eigenwerte einer Hermite'sche Matrix $H$ :

$$
\begin{aligned}
& H=V \Lambda V^{*} \\
& H H^{*}=U S V^{*} V S^{*} U^{*}=U\left(S S^{*}\right) U^{*} \\
& H^{*} H=V S^{*} U^{*} U S V^{*}=V\left(S^{*} S\right) V^{*}
\end{aligned}
$$

Damit entsprechen die Quadrate der positiven Eigenwerte $\Lambda$ von $H$ den durch SVD gewonnenen positiven Eigenwerten von $H H^{*}$ und $H^{*} H$, wobei die Spaltenvektoren von $U$ (linkssinguläre Vektoren) Eigenvektoren von $H H^{*}$ sind, die Spaltenvektoren von $V$ (rechtssinguläre Vektoren) sind Eigenvektoren von $H^{*} H$.

Im vorliegenden Fall der Interaktionsmatrix haben wir es mit Messwerten von Koordinatendifferenzen zu tun, also liegt immer eine reelle Matrix vor, deren Adjungierte in diesem Fall gleich der transponierten Matrix $A^{\mathrm{T}}$ ist:

$$
A \in \mathbb{R} \Rightarrow A^{*}=A^{\mathrm{T}} .
$$

Das Problem der Invertierung von $A$ ist nun auf das Problem der Invertierung von $S$ geschrumpft. Tatsächlich wird jedoch nur eine Pseudoinverse $A^{+}$nach

$$
A^{+}=V S^{+} U^{*}
$$

zur Lösung des 'minimum least square'-Problems berechnet, wobei $S^{+}$aus der Transponierten von $S$ hervorgeht, indem jeder von Null verschiedene Eintrag durch seinen Kehrwert ersetzt wird.

\subsubsection{Korrekturstärke und cutoff-Parameter}

Im Beobachtungsalltag trifft man oft auf Seeing-Bedingungen, die ein hohes Signal-zuRauschen-Verhältnis der WFS-Messungen nicht zulassen. Somit verlieren leichte Variationen im Messsignal ihre Eindeutigkeit, wenn sie unter dem Einfluss des Rauschens nicht 


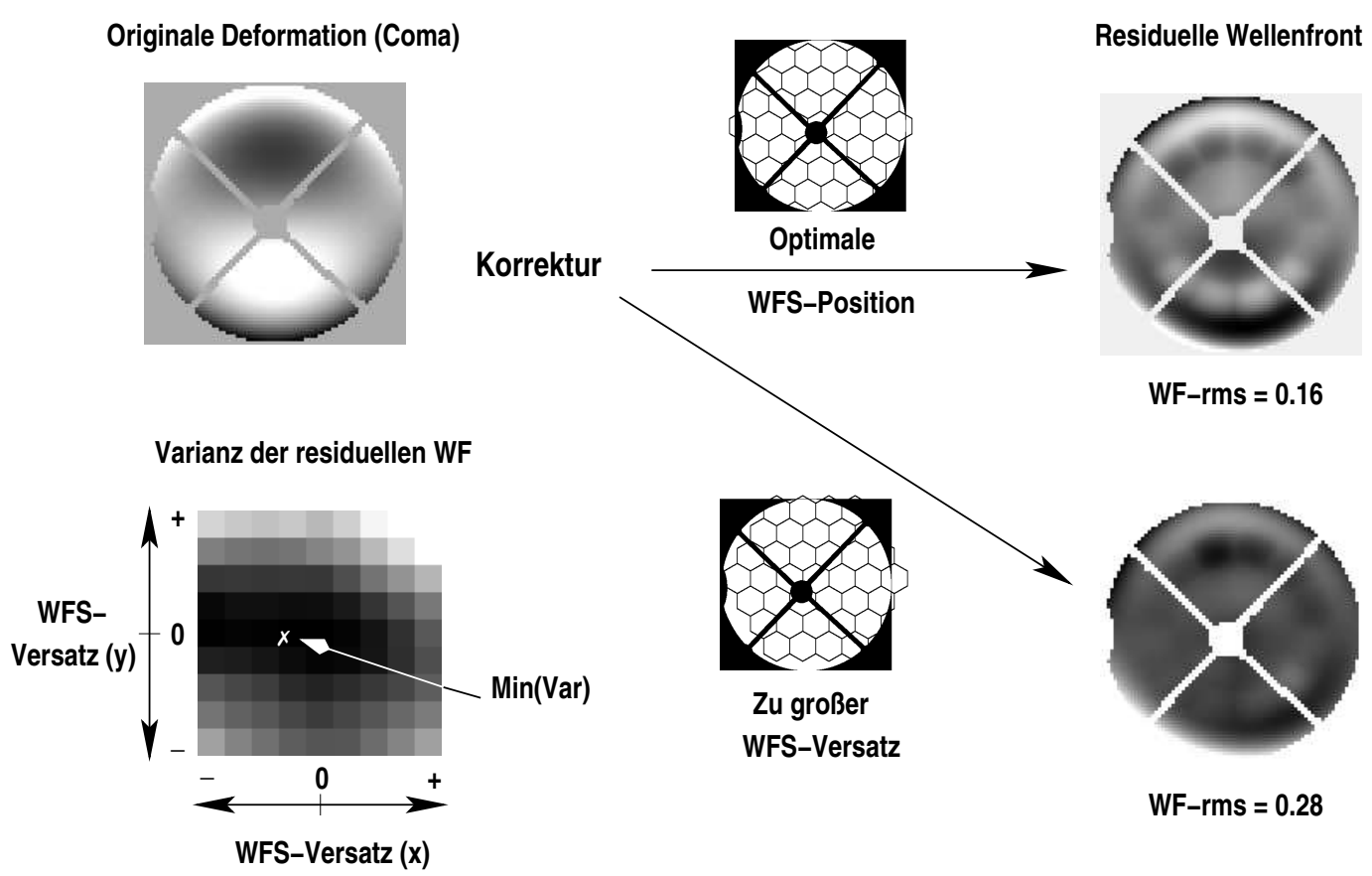

Abbildung 5.6: Zum Einfluss der gegenseitigen Ausrichtung von WFS vs. Pupille. Die optimale Abdeckung der Eintrittsöffnung durch das Lenslet-Array minimiert die Varianz des Wellenfrontfehlers nach der Korrektur, ermöglicht also optimale Korrektur. Eine ähnliche Vorgehensweise wurde zur Bestimmung der besten Werte für Verdrehung und Versatz von Sensor, Spiegeln und Pupille gegeneinander verwendet.

voneinander unterschieden werden können. Dies macht sich in der Größe der Singulärwerte bemerkbar, die ja gewissermaßen die Koordinaten im $m$ - bzw. $n$-dimensionalen Raum bilden, den die orthonormierten Spaltenvektoren von $U$ bzw. $V$ aus G1.5.9 aufspannen. Die Singulärwerte werden also ab einer gewissen Grenze, etwa $r$, so klein, dass die zugehörigen Kehrwerte in $S^{+}$dem Beitrag aus den unsicheren Messungen zu großes Gewicht verleihen. Es gilt also nun, eine untere Grenze für die Singulärwerte zu finden, einen 'cutoff'-Parameter, der effektiv den Signalbereich vom Rauschen trennt. Je nach Seeing sollte diese Grenze variabel sein, um bei besserem Seeing auch die signifikanten schwachen Signale berücksichtigen zu können. Als Set von solchen cutoff-Parametern wurden in Übereinstimmung mit dem AO-System KAOS die fünf Werte $\{0.1,0.14,0.2,0.28,0.4\}$ eingesetzt, die effektiv die Empfindlichkeit des WFS und damit auch die möglichen Korrekturniveaus kodieren.

\subsubsection{Gegenseitige Orientierung von WFS und DM}

Im Hinblick auf Optimierung der theoretisch möglichen Korrekturleistung des Systems kommt den möglichen gegenseitigen Ausrichtungen der optischen Hauptbestandteile des AO-Systems signifikante Bedeutung zu. Sowohl das hexagonale Muster des WFS wie auch die Keystone-Anordnung der DM-Elektroden lassen sich in der Bildebene der Pupille gegen selbige verdrehen oder verschieben. Verkippungen mit ihren Projektionseffekten, also etwa ovale Pupillenform etc., wurden nicht in den Parameterraum aufgenommen. 
Dies resultiert hauptsächlich in drei Variationen:

- WFS vs. Pupille

Durch die Abschattungen des Nachführspiegels können aus gewissen Subaperturen keine oder stärker verrauschte Messwerte für die WF-Neigung gewonnen werden. Besser als die teilweise Bedeckung mehrerer Subaperturen stellt sich die totale Abschattung möglichst weniger Lenslets heraus.

- DM vs. Pupille

Für eine kreisrunde Pupille ohne Obstruktionen bietet die zentrierte Positionierung des Spiegels im Strahlengang offensichtlich die größte Flexibilität des DM zur besten Darstellung der WF-Deformation. Bei Abweichung von der Kreisform und bei unsymmetrischen Abschattungen muss der optimale Bereich der Ausleuchtung auf dem DM jedoch nachjustiert werden.

- WFS vs. DM

Großen Einfluss auf die Korrekturleistung hat in dieser Liste auch die relative Position von WFS gegen DM. Dies ist bei der modalen Korrektur atmosphärischer WF nicht leicht einzusehen, wird aber bei der Kalibration deutlich, wo die durch einzelne Elektroden hervorgerufene Deformation des DM vom Lenslet-Array gesampelt wird. Hier erweist es sich als günstiger, wenn die projizierte Deformation zentriert über den Eckpunkten der Hexagone liegt. Durch die unterschiedliche Geometrie nimmt dieser Effekt mit steigender Anzahl von Spiegelelektroden und WFS-Subaperturen an Wichtigkeit ab.

Stellvertretend für die Optimierung der sechs betrachteten Freiheitsgrade von WFS und DM gegenüber der Pupille ist das Vorgehen in Abbildung 5.6 am Beispiel der Verschiebung des WFS gegenüber der Pupille illustriert. Die Kalibration wird mit der Bestimmung der Interaktions- und Kontrollmatrix für jede Verschiebung des Hexagon-Feldes (um ganzzahlige Vielfache der Pixelseitenlänge) durchgeführt und die Korrektur einer bekannten, deformierten WF mit dieser Einstellung simuliert. Im Beispiel wurde eine einzelne Zernike-Mode korrigiert und der resultierende, übrigbleibende Wellenfrontfehler als rms-Wert berechnet. Über das Maréchal-Kriterium kann damit das Strehl-Verhältnis berechnet werden. Im Beispiel beschreibt $S=0.93$ den übermäßigen Versatz des WFS und $S=0.97$ die optimale Stellung. Im Anwendungsfall ist jedoch der relative Gewinn an Abbildungsqualität durch optimale Justage der optischen Elemente neben der Korrekturempfindlichkeit von der Modenkomposition der WF und den relativen Gewichtungen der Moden abhängig.

\subsubsection{Wellenfrontkorrektur}

Zum Abschluss der eigentlichen AO-Simulation wird noch die tatsächliche Korrektur modelliert, die auf die Wellenfronten verschiedener Richtungen angewendet werden. Verglichen mit den übrigen Arbeitsschritten, gestaltet sich dies außerordentlich einfach, da der einfallenden WF nur die Wellenfrontdeformation des Spiegels aufgeprägt werden muss. Dazu bildet man einfach die Differenz aus der originalen WF $\phi_{\text {unkorr }}$ und der berechneten Spiegelform $\phi_{D M}$ und erhält die resultierende residuelle Wellenfront

$$
\phi_{\text {res }}=\phi_{\text {unkorr }}-\phi_{D M} .
$$




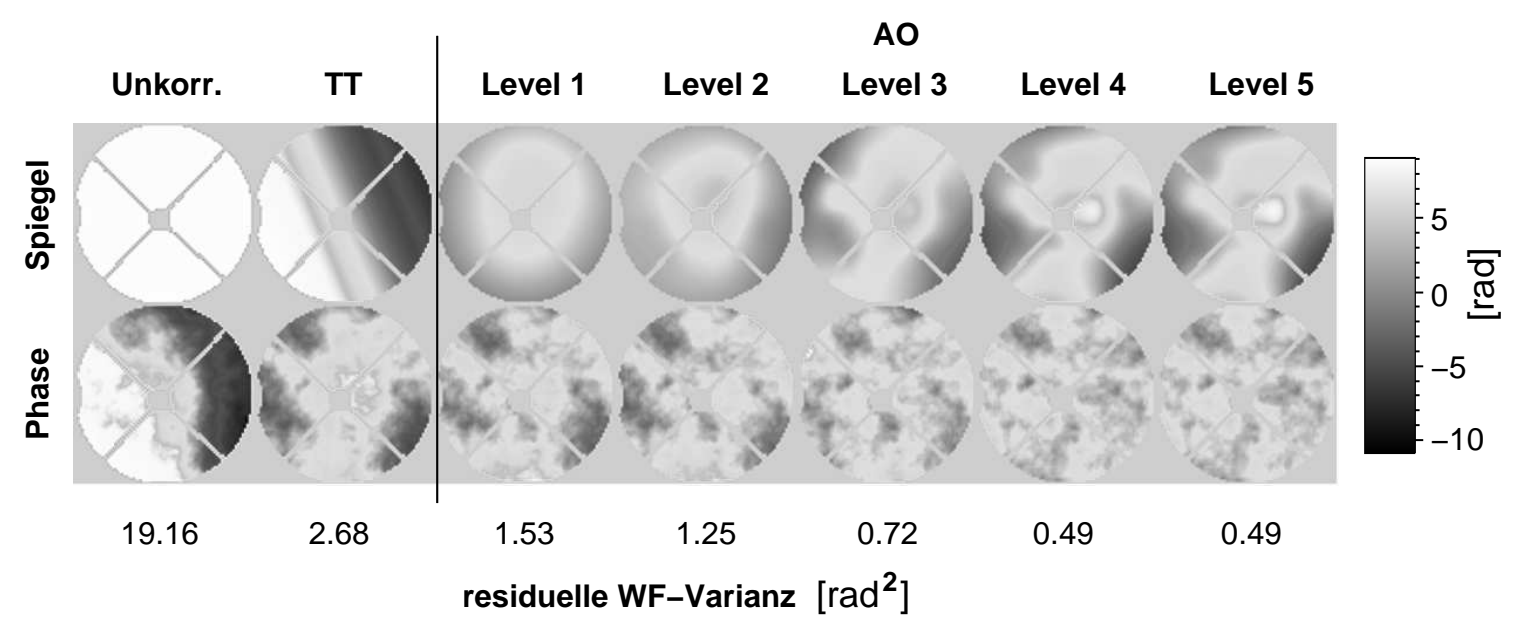

Abbildung 5.7: Beispiel zur AO-Korrektur einer Wellenfront im Simulationscode. Abgebildet sind in der oberen Reihe von links: die Eintrittspupille, die Korrekturstellung des Tip/Tilt-Spiegels und für die fünf Korrekturstärken die berechnete Form des DMSpiegels. In der unteren Reihe sind dazu die jeweiligen residuellen Wellenfronten abgebildet. Von links: Phasenschirm der unkorrigierten Wellenfront, nach TT-Korrektur, und für jede Korrekturstärke der AO nach Korrektur mit DM und TT-Spiegel. Die Grauschattierung gibt die relative Phasendiffernz in Radian an.

Hier ist wohlgemerkt auch berücksichtigt, dass die tatsächlich eingeführten optischen Weglängenunterschiede der doppelten Amplitude der Spiegeldeformation entsprechen! Da die aus der WF-Messung in Lockpoint-Richtung berechnete Spiegelform separat abgespeichert wurde, kann $\phi_{\text {unkorr }}$ sowohl die ursprüngliche WF in Lockpoint-Richtung wie auch diejenige aus einer beliebigen anderen Richtung im beobachteten Bildfeld darstellen. So kann auf diese Weise die winkelabhängige Korrektur des adaptiven optischen Systems untersucht werden: die einmal errechneten Spiegeldeformationen werden jeweils von den entsprechenden Serien von Wellenfronten abgezogen, die für eine Reihe von Winkeln zur Lockpoint-Richtung zur Simulation eines ausgedehnten Gesichtsfeldes berechnet wurden.

Die wichtigsten Elemente und Arbeitsschritte bei der Simulation der AO-Korrektur sind damit vorgestellt. Die Wellenfronten vor und nach der Korrektur werden lediglich noch zur weiteren Verwendung abgespeichert.

\subsection{Output - Übertragungsfunktionen}

Da wir an konkreten Messwerten betreffend der Verbesserung der Bildqualität bei Einsatz einer Adaptiven Optik interessiert sind, müssen aus den korrigierten Wellenfronten zugehörige Punktverbreiterungsfunktionen errechnet werden, aus denen für die Fälle 'kurze' oder 'lange' Belichtungszeit Strehl-Zahlen und Halbwertsbreiten abgeleitet werden können.

Auf dem Weg dorthin bietet sich schon allein rechnerisch die Gelegenheit, auch die zuge- 
hörige Fourier-Darstellung, also die OTF mitzuberechnen, deren Gebrauch bei Langzeitund Kurzzeitbelichtung schon in Abschnitt 3.4.2 eingeführt wurden. Gerade im Hinblick auf die Anwendbarkeit der Speckle-Bildrekonstruktion in Verbindung mit Adaptiver Optik wird der Mittelwert des Betragsquadrates, auch Speckle-Transferfunktion (STF) genannt, zur Kennzeichnung einer 'typischen' Kurzzeitbelichtung interessant.

\subsubsection{PSF, Übertragungsfunktionen und Strehl-Verhältnis}

Die konkrete Bestimmung der PSF und STF erfolgt durch Implementierung der Integralausdrücke aus Gleichung 2.23, also mit der üblichen Propagationsgleichung für ein optisches Feld U in der Pupille zur Berechnung der PSF im Fernfeld:

$$
P S F(\boldsymbol{\alpha})=|\mathcal{F}\{U(\boldsymbol{\xi})\}|^{2}(\boldsymbol{\alpha} / \lambda)
$$

bzw. in der Fokalebene mit $\boldsymbol{\alpha}=\boldsymbol{x} / f$

$$
P S F(\boldsymbol{x})=\frac{1}{A}\left|\iint_{\text {Pupille }} W_{\text {Pup }}(\xi) \phi_{\text {res }}(\xi) e^{-i \frac{2 \pi}{\lambda f} \boldsymbol{x} \boldsymbol{d}} \mathrm{d} \boldsymbol{\xi}\right|^{2} .
$$

Hier spiegelt der zweite Ausdruck mit Ortskoordinaten $\boldsymbol{\xi}$ bzw. $\boldsymbol{x}$ für Pupille bzw. Bildebene die implementierte Form klarer wider. Der Vorfaktor A normiert auf den Gesamtenergiegehalt, also auf das Integral über die PSF. Die Berechnung der STF als ein Mittelwert vieler momentaner Übertragungsfunktionen diktiert nach dem Wiener-KhinchineTheorem (siehe Anhang B) eine Rücktransformation der PSF zur OTF, die auf Grund der Energienormierung der PSF am Frequenznullpunkt auf eins normiert ist:

$$
O T F(\boldsymbol{s})=\mathcal{F}^{-1}\{P S F(\boldsymbol{\alpha})\}(\boldsymbol{s})
$$

bzw. mit Koordinatendifferenzen $\Delta=\left(\boldsymbol{\xi}_{1}-\boldsymbol{\xi}_{2}\right)$ in der Pupillenebene

$$
O T F(\boldsymbol{\Delta})=\iint P S F\left(\frac{\boldsymbol{x}}{\lambda f}\right) e^{i \frac{2 \pi}{\lambda f} \boldsymbol{x}\left(\boldsymbol{\xi}_{\mathbf{1}}-\boldsymbol{\xi}_{\mathbf{2}}\right)} .
$$

Dabei gilt $s=\frac{\Delta}{\lambda}$.

$\mathrm{Zu}$ jeder der $\mathrm{N}$ Realisierungen einer WF wird die zugehörige momentane OTF und PSF berechnet und abgespeichert. Sie beschreiben einzelne Kurzzeitbelichtungen eines astronomischen Objektes durch die turbulente Atmosphäre und sind als Beispiel aus den in Abb. 5.7 gezeigten Wellenfronten berechnet und in Abb. 5.8 visualisiert.

Die gesuchte Speckle-Transferfunktion ergibt sich als das mittlere Betragsquadrat der OTFs

$$
\operatorname{STF}(\boldsymbol{s})=\frac{1}{N} \sum_{i=1}^{N}\left|O T F_{i}(s)\right|^{2},
$$

sodass sie also bei etwa konstanten Seeing-Bedingungen gewissermaßen einen 'typischen Schnappschuss' beschreibt. Zusätzlich wird noch eine künstliche Langzeitbelichtung simuliert, indem alle errechneten komplexwertigen OTFs direkt gemittelt werden:

$$
O T F_{L E}=\frac{1}{N} \sum_{i=1}^{N} O T F_{i}
$$




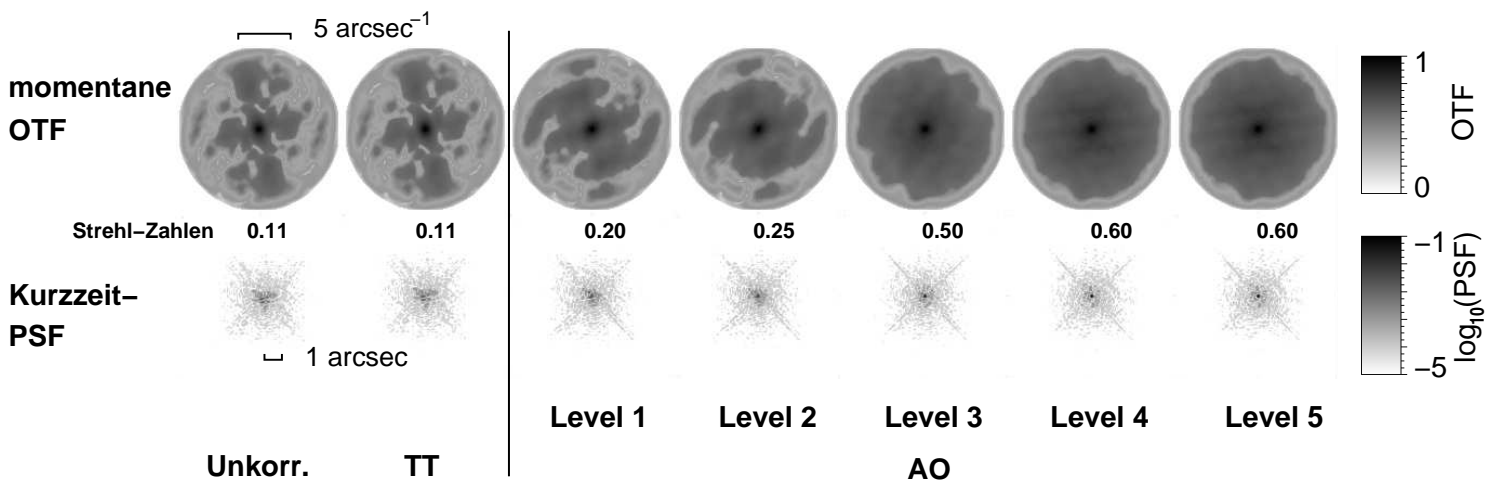

Abbildung 5.8: Beispiele für durch den Simulationscode errechnete OTFs und PSFs aus einer einzelnen AO-korrigierten Wellenfront. Abgebildet sind in der oberen Zeile die momentanen optischen Übertragungsfunktionen, darunter die zugehörigen Punktverbreiterungsfunktionen. Von links nach rechts nimmt die WF-Korrektur zu: ganz links die Größen ohne Korrektur, dann Bildstabilisierung durch den Tip/Tilt-Spiegel (TT) und davon rechts die eigentliche Korrektur durch den deformierbaren Spiegel (DM).

Die verbleibende, wichtige Kenngröße ist die Strehl-Zahl. Sie lässt sich einfach aus dem Verhältnis der Maxima von aktueller PSF und gespeicherter Ideal-PSF ablesen. Dies ist möglich, da die beugungsbegrenzten Größen schon zu Beginn jeder simulierten Aufnahmeserie aus einem über die Eintrittspupille konstanten Phasenschirm berechnet werden.

Beschreibung der Abb. 5.9 In Abb. 5.9 ist das Ergebnis der Simulationsrechnungen am Beispiel der Wellenfrontserie gezeigt, aus der auch der Phasenschirm entnommen wurde, der zur Berechnung der momentanen Größen benutzt wurde, die in den Abbildungen 5.7 und 5.8 gezeigt wurden. Die Serie aus 100 Wellenfronten wurde mit einem FriedParameter von $10 \mathrm{~cm}$ bzw. für ein Seeing von $1^{\prime \prime}$ errechnet. Dargestellt sind (bitte Blatt um $90^{\circ}$ im Uhrzeigersinn drehen) in der oberen Reihe die PSFs der Langzeit-Belichtung, in der zweiten Zeile die zugehörigen OTFs und darunter die Speckle-Transferfunktionen. Von links nach rechts nimmt die AO-Korrektur zu, von unkorrigiert über TT-kompensiert und die verschiedenen Grade der Empfindlichkeit für den DM (Level 1 bis 5). Die Korrekturen sind für die Lockpunkt-Richtung und ohne Dekorrelationseffekte berechnet.

\subsubsection{Parameterraum}

Die ursprüngliche Idee der Erstellung einer Bibliothek von STFs mit verschiedenen Parameterkombinationen erwies sich als entweder zu aufwändig (bei 'Vollständigkeit') oder aber schwierig abzugrenzen: Welche sind die wichtigsten Parameter, auf die man sich beschränken muss? Wie im Abschnitt 5.2 schon argumentiert, erwachsen die beiden Turbulenzschichten aus einer Minimalannahme und mit ihnen sind jeweils charakterisierende Größen verbunden: der lokale Fried-Parameter, relative Höhe, äußere Turbulenzskalen und angenommene Windstärke. Von Seiten der AO-Simulation sind zunächst die Korrekturniveaus und Anzahl der Blickrichtungen zu nennen. Welches sind dabei die möglichst typischsten Parameterwerte? Diese sollten in genügender 'Auflösung' untersucht werden, 
5.4 Output - Übertragungsfunktionen

\begin{tabular}{|l|l|l|}
\hline Parameter & Wertebereich & Schrittweite \\
\hline \hline Höhe der oberen Turbulenzschicht & $8000-10000[\mathrm{~m}]$ & $500[\mathrm{~m}]$ \\
\hline Fried-Parameter unten & $5-25[\mathrm{~cm}]$ & $1[\mathrm{~cm}]$ \\
\hline Fried-Parameter oben & $30-150[\mathrm{~cm}]$ & $15[\mathrm{~cm}]$ \\
\hline Windstärke unten & $0-20[\mathrm{~m} / \mathrm{s}]$ & $5[\mathrm{~m} / \mathrm{s}]$ \\
\hline Windstärke oben & $0-100[\mathrm{~m} / \mathrm{s}]$ & $20[\mathrm{~m} / \mathrm{s}]$ \\
\hline Windrichtung (unten u. oben) & $0-90\left[{ }^{\circ}\right]$ & $45\left[{ }^{\circ}\right]$ \\
\hline Korrekturniveaus (AO) & - & 7 \\
\hline Blickrichtung (zum Lockpunkt) & $0-60\left[^{\prime \prime}\right]$ & 10 \\
\hline \hline Anzahl der STF-Arrays: & $64 \times 64$ bzw. $128 \times 128$ & $\begin{array}{l}2.6 \cdot 10^{6} \\
(10 \mathrm{bzw} .40 \mathrm{~GB})\end{array}$ \\
\hline
\end{tabular}

Tabelle 5.1: Zusammenstellung wichtiger Parameter für die Modellierung typischer Beobachtungsbedingungen. Jeweils mit dem für die Größen in Simulationen angenommenen wichtigsten Wertebereich und durchschnittliche Schrittweiten. Für wellenlängenabhängige Parameter wurde von $\bar{\lambda}=500 \mathrm{~nm}$ ausgegangen.

also der Parameterraum in diesen Richtungen ausreichend fein gesampelt werden. Zusätzlich wären noch technische Besonderheiten und ungünstige Anordnungen im optischen Aufbau zu berücksichtigen, wie etwa Abschattungen der Eintrittspupille, wodurch nicht alle Subaperturen des WFS benutzt werden können. Dieser Fall tritt beim VTT beispielsweise auf, wenn früh am Morgen relativ horizontnah beobachtet wird. Der große Einfallswinkel auf die runden Coelostatenspiegel verursacht in der Projektion eine ellipsenförmige Begrenzung des Strahlbündels, das zusätzlich noch durch die kreisförmige Eintrittsöffnung im Vakuumtank begrenzt wird. Für jede Kombination dieser Werte sollte eine STF als Datenmatrix von etwa 128 Pixeln Seitenlänge vorliegen, um bei förmlich jeder zu erwartenden Beobachtungsbedingung eine möglichst akkurate Intensitätsverstärkung bei einer eventuellen Speckle-Rekonstruktion anwenden zu können.

Angesichts der Größe des Parameterraumes und der sich daraus ergebenden (recht unhandlichen) Fülle an abzuspeichernden Daten erscheint es trotz sinkender Speicherplatzkosten grundsätzlich sinnvoller zu sein, ausreichende Informationen zur Modellierung adäquater Übertragungsfunktionen während der Beobachtungen zu sammeln und eine STF jeweils speziell für einen gegebenen Datensatz zu berechnen. Ein Ansatz, der wiederum durch wachsende Rechnerleistung an Attraktivität gewinnt. 

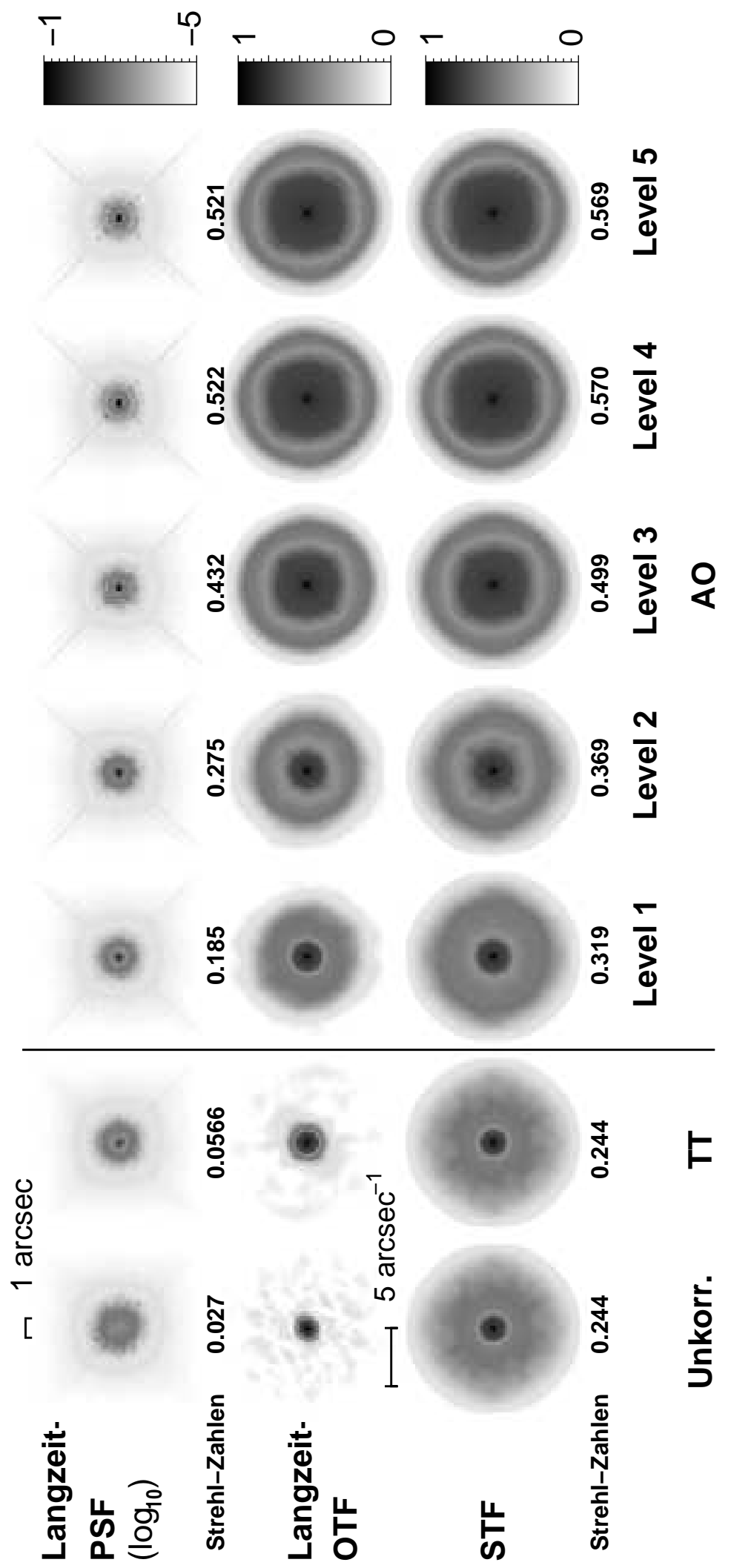

Abbildung 5.9: Beispiel für die gemittelten 2D-Größen, die vom Simulationscode berechnet werden $\left(r_{0}=10 \mathrm{~cm}\right)$. Weitere Beschreibungen sind auf Seite $68 \mathrm{zu}$ finden. 


\section{Beobachtungsmaterial}

Der Konzeption der Simulation lag von Anfang an die Idee zu Grunde, sich möglichst nah an den tatsächlichen Gegebenheiten des benutzten Instruments zu bewegen, um im günstigsten Falle auch realistische Aussagen zur Systemleistung ableiten zu können. Zu diesem Zweck wurden auch eigene Beobachtungen zur Gewinnung von experimentellen Daten durchgeführt. Deshalb soll zunächst kurz auf relevante technische Größen des Teleskops und des adaptiven optischen Systems eingegangen werden. Beschreibungen zum Datenmaterial schließen sich nach Art und Verwendung an diesen Teil an.

\subsection{Instrumentelles}

\subsubsection{Das Vakuum-Turm-Teleskop}

Der schematische Strahlengang des Vakuum-Turm-Teleskops (VTT) mit seinem AOSystem KAOS ist in Abbildung 6.1 skizziert. Die beiden Coelostatenspiegel lenken das Licht in den ortsfesten, vertikalen Vakuumtank. Dort fällt das Licht auf den $70 \mathrm{~cm}$ Hauptspiegel, durch den ein konvergenter $f / 66$ Strahlengang entsteht. Die Bildskala im Primärfokus $F 1$ beträgt dann 4 ".6/mm. Der Kollimatorspiegel bildet dann die Eintrittspupille des Teleskops auf den $50 \mathrm{~mm}$ durchmessenden Zentralbereich des deformierbaren Spiegels der Adaptiven Optik ab. Zuvor trifft das Licht noch auf den Tip/Tilt-Spiegel, der die globale Bildbewegung kompensiert. Somit bleibt dem DM nur die Aufgabe, die Bildunschärfe zu korrigieren. Im Strahlengang nach der Wellenfrontkorrektur fällt ein kleiner Teil des Lichts durch einen dichroitischen Strahlteiler auf den Wellenfrontsensor. Der größte Teil des Lichtes wird jedoch zum wissenschaftlichen Instrument gespiegelt. Das Verkippen dieses Strahlteilers ermöglicht z. B. ein Abscannen des Sonnenbildes mit dem Spektrographen. Der Kameraspiegel erzeugt anschließend ein korrigiertes Bild im Fokus F2 mit dem originalen Abbildungsmaßstab von $4 . .6 / \mathrm{mm}$.

\subsubsection{KAOS}

KAOS ist inzwischen ein Standardinstrument für die Beobachtung bei hoher räumlicher Auflösung und kann sämtliche wissenschaftlichen Instrumente bedienen. Im Gegensatz zu AO-Systemen an anderen Teleskopen kann KAOS ohne Einschränkung auf der relativ kontrastarmen solaren Granulation betrieben werden und erreicht bei gewissen Ausgangsbedingungen (Seeing besser als 0'.8) sogar beugungsbegrenzte Bildqualität (StrehlZahl > 0.3). Nach dem Rayleigh-Kriterium liegt die theoretisch mögliche Auflösung mit $\lambda=500 \mathrm{~nm}$ bei $1.22 \lambda / D=00^{\prime} 18$. Eine leicht zu bedienende graphische Oberfläche 


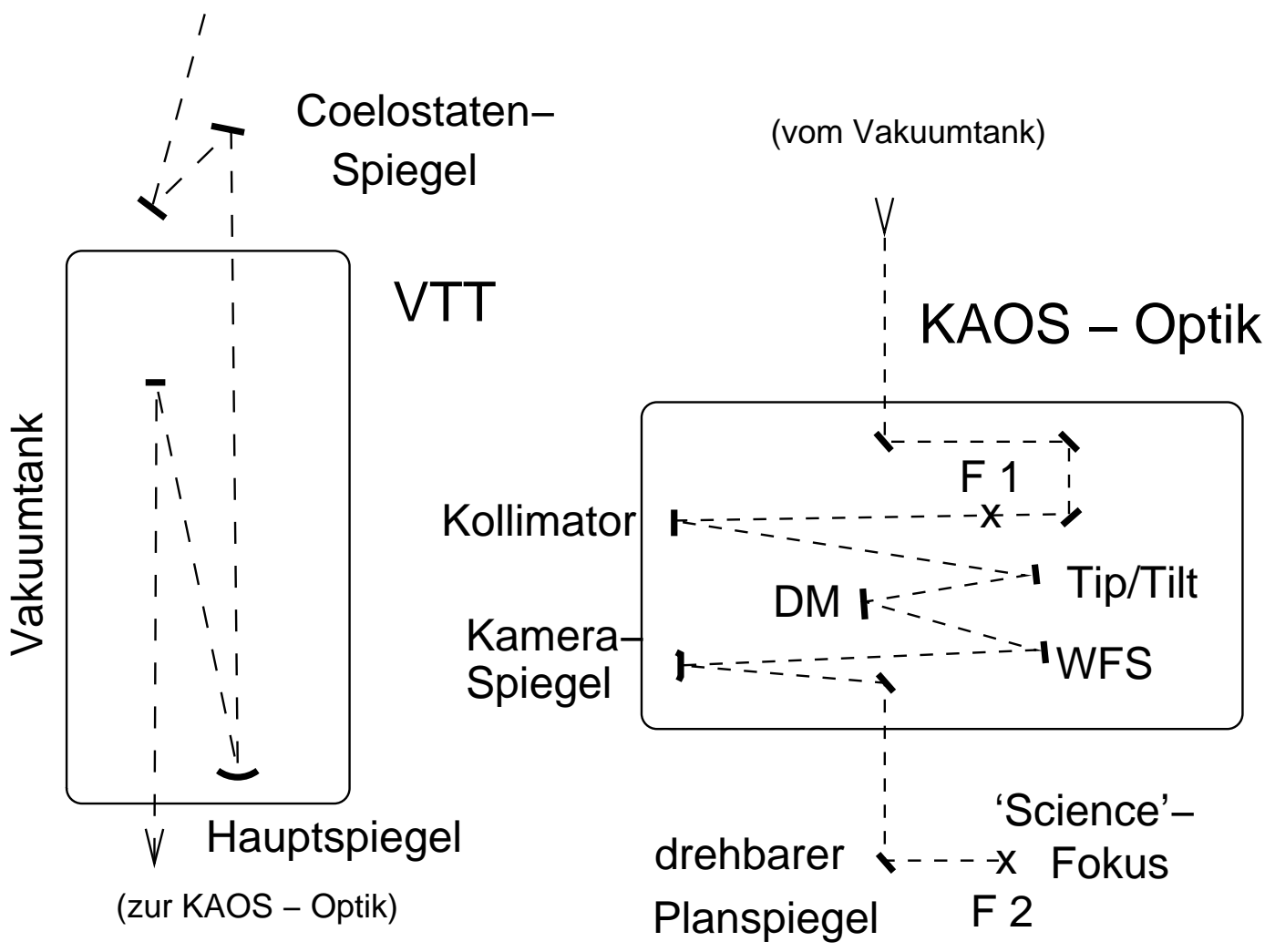

Abbildung 6.1: Die Abbildung zeigt den optischen Aufbau des VTT mit seiner Adaptiven Optik. Detaillierte Beschreibung siehe Text.

macht den Einsatz von KAOS sehr benutzerfreundlich. Die wichtigsten Parameter von KAOS sind in Tabelle 6.1 aufgelistet.

\begin{tabular}{|c|c|}
\hline Korrektur-Freiheitsgrade & maximal 27 (etwa Zernike-Moden) \\
\hline Korrektur-Frequenz & $955 \mathrm{~Hz}$ \\
\hline Bandbreite & $60 \mathrm{~Hz}$ (geschlossener Regelkreis) \\
\hline Betriebsbereich (Seeing) & $\begin{array}{l}\text { 1".5 (für stabilen Betrieb) } \\
\text { 0!.8 (für beugungsbegrenzte Beobachtungen) }\end{array}$ \\
\hline Windgeschwindigkeit & maximaler Mittelwert um $12 \mathrm{~m} / \mathrm{s}$ \\
\hline
\end{tabular}

Tabelle 6.1: Die wichtigsten Rahmendaten zum Betrieb des Adaptiven Optischen Systems KAOS. 


\subsubsection{DALSA Kamera}

Für die Aufnahme der Beobachtungsdaten und im WFS wurden die Speckle-Kameras DALSA-CA-D7 und DALSA-CCD-1M30 ('neu') verwendet. Die wichtigsten Spezifikationen sind in Tabelle 6.2 aus den Datenblättern der Kameras zusammengestellt. Die in der Beobachtungskampagne 2005 zum ersten Mal verwendete neue Kamera konnte trotz besserer nomineller Aufnahmeraten (15 bis 30 Bilder/s) aufgrund einer Reihe von Fehlern in der Steuerungssoftware nur bei der angegebenen Bildrate verwendet werden.

\begin{tabular}{|l|l|}
\hline Kamerabezeichnung & DALSA-CA-D7 1024T und DALSA-CCD 1M30 \\
\hline Pixel & $12 \mu \mathrm{m} \times 12 \mu \mathrm{m}$ \\
\hline Dynamischer Bereich & 12 bit/Pixel, aufgezeichnet als 16 bit long-integer \\
\hline Bildrate & $7-8$ Bilder $/ \mathrm{s}(125 \mathrm{~ms}+$ Belichtungszeit $)$ \\
\hline Empfindlichkeit & front side illuminated \\
\hline Wellenlänge & $430.5 \pm 1.5 \mathrm{~nm}(\mathrm{G}-$ Band $)$ \\
\hline Belichtungszeit & $5-10 \mathrm{~ms}$ \\
\hline Bildfeldskala & $0.056^{\prime \prime} /$ Pixel \\
\hline Bildgröße & $1024 \times 1024$ Pixel $\left(58^{\prime \prime} \times 58^{\prime \prime}\right)$ \\
\hline
\end{tabular}

Tabelle 6.2: Technische Spezifikationen der verwendeten DALSA-Kameras

\subsection{Beobachtungen am VTT}

\subsubsection{Speckle-Daten}

Zur späteren Speckle-Rekonstruktion werden jeweils genügend viele 'Schnappschüsse' durch die turbulente Atmosphäre benötigt, um ergodische Bedingungen zu erreichen und eine gute Schätzung für den Mittelwert der Distorsionen ableiten zu können. Somit dokumentieren diese Folgen von jeweils 100 Kurzzeitaufnahmen ('Bursts') die zeitliche Entwicklung solarer Strukturen, hier im sogenannten G-Band. Das Fraunhofer'schen G-Band ist ein molekulares Absorptionsband des $\mathrm{CH}$ im solaren Spektrum bei Wellenlängen um $\lambda \approx 430$ unitnm. Es zeigt dichtgedrängte starke Absorptionslinien, die durch elektronische Übergänge zwischen Rotations- und Vibrationszuständen dieser Moleküle verursacht werden. Die Photosphäre erscheint bei diesen Wellenlängen sehr kontrastreich. Dies liegt vor allem an den sogenannten 'G-band bright points', auffallend hellen Strukturen, die bevorzugt im intergranularen Bereich auftreten. Ihre Intensitätswerte liegen deutlich über der Kontinuumsintensität (Größenordnung 50\%) und werden als Strahlungssignatur kleinskaliger ( $\varnothing \leq 150 \mathrm{~km}$ ) magnetischer Flussröhren gedeutet. Neben der unmittelbaren Untersuchung morphologischer und dynamischer Eigenschaften dieser kleinsten, be- 
obachteten solaren Flusskonzentrationen ermöglichen diese Strukturen ein hohes Signalzu-Rauschen-Verhältnis bei der Bestimmung von Größen wie der PSF aus den Bilddaten selbst. Durch Beugung an der Teleskopöffnung sind diese Strukturen im Falle des VTT nicht auflösbar, also haben sie auf Grund ihrer räumlichen Ausdehnung angenähert Punktlichtquellencharakter, wenn auch etwas durch die Umgebungshelligkeit kontaminiert. Somit eignet sich diese Wellenlänge hervorragend für Beobachtungen zur Unterstützung der hier durchgeführten Analysen der Abbildungsqualität durch Speckledaten eines kontinuierlich ausgedehnten astronomischen Objektes.

Die in den Tabellen 6.3 und 6.4 dargestellten (Teil-)Protokolle der Beobachtungen sind absichtlich verkürzt dargestellt; hier soll jedoch nur ein ungefährer Eindruck von der Art und Menge der Daten gewonnen werden. Bei der zeitlichen Zuordnung zu den im Folgenden beschriebenen AO-Daten muss auf die Erstellungszeit der Original-datei zurückgegriffen werden, da ein Fehler im Kameratreiber der neuen DALSA 1M30 die Zeit nicht, wie sonst üblich, in den FITS-Header schrieb.

\subsubsection{AO-Mitschriften}

\begin{tabular}{|l|c|c|l|}
\hline Typ & Anzahl & Format & Bedeutung \\
\hline \hline Aktuatorsignale & 38 & double & proportional zur angelegten Spannung \\
\hline Shiftwerte & 74 & double & Bildversatz (x,y) [Pixel] \\
\hline Zernike-Moden & 27 & double & Zernike-Koeffizienten der WF [rad] \\
\hline Rekonstruktor & 1 & long int & $\begin{array}{l}\text { Kennzahl des Korrekturniveaus bzw. } \\
\text { der verwendeten Rekonstruktionsmatrix }\end{array}$ \\
\hline Spiegelmoden & 1 & long int & Anzahl korrigierter DM-Eigenmoden \\
\hline$r_{0}$ (unkorrigiert) & 1 & double & $\begin{array}{l}\text { Geschätzter atmosphärischer } \\
\text { Fried-Parameter vor der AO-Korrektur }\end{array}$ \\
\hline$r_{0}$ (korrigiert) & 1 & double & $\begin{array}{l}\text { Effektiver Fried-Parameter } \\
\text { mit AO-Korrektur }\end{array}$ \\
\hline
\end{tabular}

Tabelle 6.3: Zusammenstellung der wichtigsten Typen von protokollierten Parametern der AO-Aktivität.

Daten bezüglich der AO-Aktivität während der Aufnahme von Speckle-Bilddaten sind in zwei Beobachtungskampagnen gespeichert worden. Wichtige Mitschriften aus der Kampagne 2004 waren jedoch nicht lesbar, da ein Fehler in der Speicherroutine vorlag. Der Versuch einer Rekonstruktion durch entsprechendes Angleichen der Leseroutine an den Fehler schlug nichtsdestoweniger fehl, da die Datenvektoren zu diesem Zeitpunkt nicht in einheitlicher Länge notiert wurden. Somit konnte nicht mehr mit Sicherheit auf die WFS-Messungen rückgeschlossen werden. 
Es liefern also nur die Daten aus dem Jahr 2005 brauchbare Information für die Analyse und Weiterbehandlung der betreffenden Bildserien. Dabei ist auf das 'Uhrenproblem' hinzuweisen. Die Genauigkeit der zeitlichen Zuordnung bleibt bei der Erstellung der AOProtokoll-Dateien im Sekunden-Bereich, obwohl mit einer Frequenz von 955 Hz gemessen und mitgeschrieben wurde. Man muss also interpolieren um zu einer genaueren Zeitangabe zu kommen.

\begin{tabular}{|c|c|c|}
\hline DALSA-Burst & Bemerkung & Datum \\
\hline $1 \ldots 139$ & $\begin{array}{l}200 \text { Bilder/Burst (10 ms) gutes Seeing } \\
\text { Letzter Burst abgebrochen }\end{array}$ & 18. April 2005 \\
\hline $140 \ldots 400$ & $\begin{array}{l}\text { Ab 11.00h, mit AO-Protokoll } \\
\text { Lücken bei 392, 398, } 400\end{array}$ & \\
\hline 401,402 & Target & \\
\hline $403 \ldots 405$ & Dark & \\
\hline $\begin{array}{l}406 \ldots 408 \\
409 \ldots 411\end{array}$ & $\begin{array}{c}\text { dreimal Gain } 3 \text { (wie Beobachtungen) } \\
\text { dreimal Gain } 5\end{array}$ & \\
\hline $1 \ldots 11$ & $\begin{array}{c}\text { Flatfields, Darks, Defokus FF } \\
200 \text { Bilder/Burst (10 ms), Gain } 1\end{array}$ & 22. April 2005 \\
\hline $12 \ldots 99$ & $\begin{array}{l}\text { verschiedene MCAO-Testaufnahmen } \\
\text { FF bei } 22,23 \text {; defokussiert: } 29,30\end{array}$ & (ab 12:50h) \\
\hline $100 \ldots 104$ & $\begin{array}{l}\text { Normale AO und MCAO } \\
\text { Vollständige AO-Protokolle. }\end{array}$ & \\
\hline $105 \ldots 109$ & $\begin{array}{l}\text { Ground layer (GL) AO mit KAOS-Daten } \\
\text { (WFS } 2 \text { und TT+DM 1) }\end{array}$ & \\
\hline $110 \ldots 119$ & $\begin{array}{l}\text { Low-order (LO) MCAO mit KAOS-Daten } \\
\text { (WFS } 2 \text { und TT + DM } 1+\text { DM } 2)\end{array}$ & \\
\hline $\begin{array}{l}128 \ldots 130 \\
131 \ldots 132 \\
133 \ldots 135\end{array}$ & $\begin{array}{c}\text { FF defokussiert }+ \text { Teleskop-Bewegung } \\
\text { FF fokussiert } \\
\text { Dark }\end{array}$ & \\
\hline$\sim 200$ & Weitere MCAO-Tests & 24. April 2005 \\
\hline
\end{tabular}

Tabelle 6.4: Protokolle zur KAOS-unterstützten Speckle-Beobachtungen im April 2005. 


\subsection{Datenmaterial vom SST}

Zusätzlich zum Material vom VTT bot sich die Gelegenheit, den Einfluss der Adaptiven Optik auf die Speckle-Rekonstruktion auch mit Bilddaten vom schwedischen SST (Swedish 1-m Solar Telescope) zu untersuchen. Die Daten wurden von meinen Kollegen Dr. K. Puschmann vom Institut für Astrophysik, Göttingen, und Dr. M. Sobotka, Ondřejov, Tschechische Republik, während einer über das OPTICON Trans-National Access Program von der Europäischen Union finanzierten Beobachtungskampagne gewonnen. Eine der am 21. Juni 2004 gewonnenen Zeitserien wurde für die durchgeführte Analyse ausgewählt. Abgebildet wurde zum Einen bei der Wellenlänge $4305.6 \AA$, also im Fraunhofer'schen G-Band, was eine theoretisch mögliche räumliche Auflösung von 0'1 nach dem Rayleigh-Kriterium zulässt. Außerdem wurde noch simultan an weiteren Wellenlängen beobachtet, bei denen das Sonnenspektrum ein Kontinuum aufweist.

\begin{tabular}{|l|l|}
\hline Datum, Zeit & 21. Juni 2004, 13:22 UT bis 13:30 UT \\
\hline Target & Aktive Region NOAA AR0636 \\
\hline Koordinaten & $6^{\circ} S, 20^{\circ} E$ (terrestrisches Bezugssystem) \\
\hline \hline Zeitserie & 100 Bilder/Burst, 2 Burst/min \\
\hline Aufnahmerate & 5.5 Bilder/s \\
\hline Belichtungszeit & 10.7 ms \\
\hline Wellenlängen & $4305.6 \AA, 4507 \AA, 6020 \AA$ \\
\hline Spektrale Transmissionsbreite & $11.56 \AA$ \\
\hline \hline Kamerabezeichnung & KODAK Megaplus $1.6 \mathrm{i}$ \\
\hline Detektor & Kodak KAF-1602, solid-state CCD \\
\hline Auslesemodus & 'Progressive Scan' \\
\hline Pixelgröße & $9 \mu \mathrm{m} \times 9 \mu \mathrm{m}$ \\
\hline Pixelskala & $0.04{ }^{\prime \prime} /$ Pixel \\
\hline Dynamischer Bereich & 10 bit/Pixel \\
\hline Bildgröße & $1536 \times 1024$ Pixel $\left(61^{\prime \prime} 44 \times 40^{\prime \prime} 96\right)$ \\
\hline
\end{tabular}

Tabelle 6.5: Wichtigste Daten der Beobachtungskampagne am schwedischen 1-m Sonnenteleskop. 


\section{Ergebnisse}

\subsection{Parameterstudien zu Simulationsrechnungen}

Als Grundlage für alle angestellten Vergleiche und Anwendungen bilden die Ergebnisse der Simulationsrechnungen den Schwerpunkt dieser Arbeit. Auf die Größe des Parameterraumes wurde schon bei der Erklärung der teilweise von mir entwickelten Programmpakete eingegangen. Hier sollen nun die Abhängigkeiten von den wichtigsten Parametern präsentiert werden. Dabei wird als Kriterium für die erreichte Abbildungsqualität die in Gleichung 3.30 eingeführte Strehl'sche Definitionshelligkeit verwendet, also das Verhältnis der Spitzenintensität der tatsächlichen PSF zur theoretisch erreichbaren Intensität im beugungsbegrenzten Fall. Dabei verwenden wir jedoch jeweils die über 100 (unabhängige) Realisationen gemittelte PSF, entsprechend einer Langzeitbelichtung von der Größenordnung einer Sekunde, wie im Abschnitt über die AO-Korrektur erklärt wurde. Das Strehl (Strehl-Zahl) kann aber auch aus den Fouriertransformierten der Größen berechnet werden und entspricht dann dem Verhältnis der über die entsprechenden Frequenzen integrierten Übertragungsfunktionen. Da uns, im Hinblick auf die Anwendung der Ergebnisse in der Speckle-Rekonstruktion, insbesondere die STFs interessieren, die der Programm-Code liefert, wird oft das entsprechende Strehl dieser Größe dargestellt. Als Verhältnis von PSFs würde dies dann einer 'typischen Kurzzeitbelichtung' bei gegebener AO-Kompensation entsprechen, wie sie bei der Speckle-Interferometrie modelliert werden muss. Die entsprechenden Zahlenwerte sind naturgemäß für diesen letzteren Fall größer, als bei der Langzeitbelichtung, da der Mittelungseffekt der Intensitäten nicht stattfindet. Mit dem Einsatz der Adaptiven Optik nähern sich beide Werte auf einem meist höheren Niveau aneinander an, abhängig von der Kombination der betrachteten Parameter.

\subsubsection{Seeing und Korrekturgrad}

Der wichtigste Parameter bei Beobachtungen, gerade was die Stabilität der AO-Korrektur betrifft, ist wohl die allgemeine Turbulenzstärke in der Atmosphäre. Sie hatte schon im Korff'schen Modell für die STF-Berechungen als einziger Parameter eine zentrale Bedeutung, die sie auch bei Einsatz der AO behält, bestimmt sie doch die Ausgangssituation und Rahmenbedingungen für unsere Bemühungen, die Bildqualität zu verbessern. Für die Simulation wurde jedoch ein Atmosphärenmodell aus zwei getrennten Schichten angenommen, die durch jeweils einen eigenen Fried-Parameter charakterisiert werden. Deshalb ist es hier möglich, Variationen in der Kombinationen der beiden Größen zu betrachten. Außerdem betrachten wir aber auch noch einen anderen wichtigen Parameter, in dessen Abhängigkeit die Verbesserung der Abbildungsqualität untersucht wurde, die Kompen- 

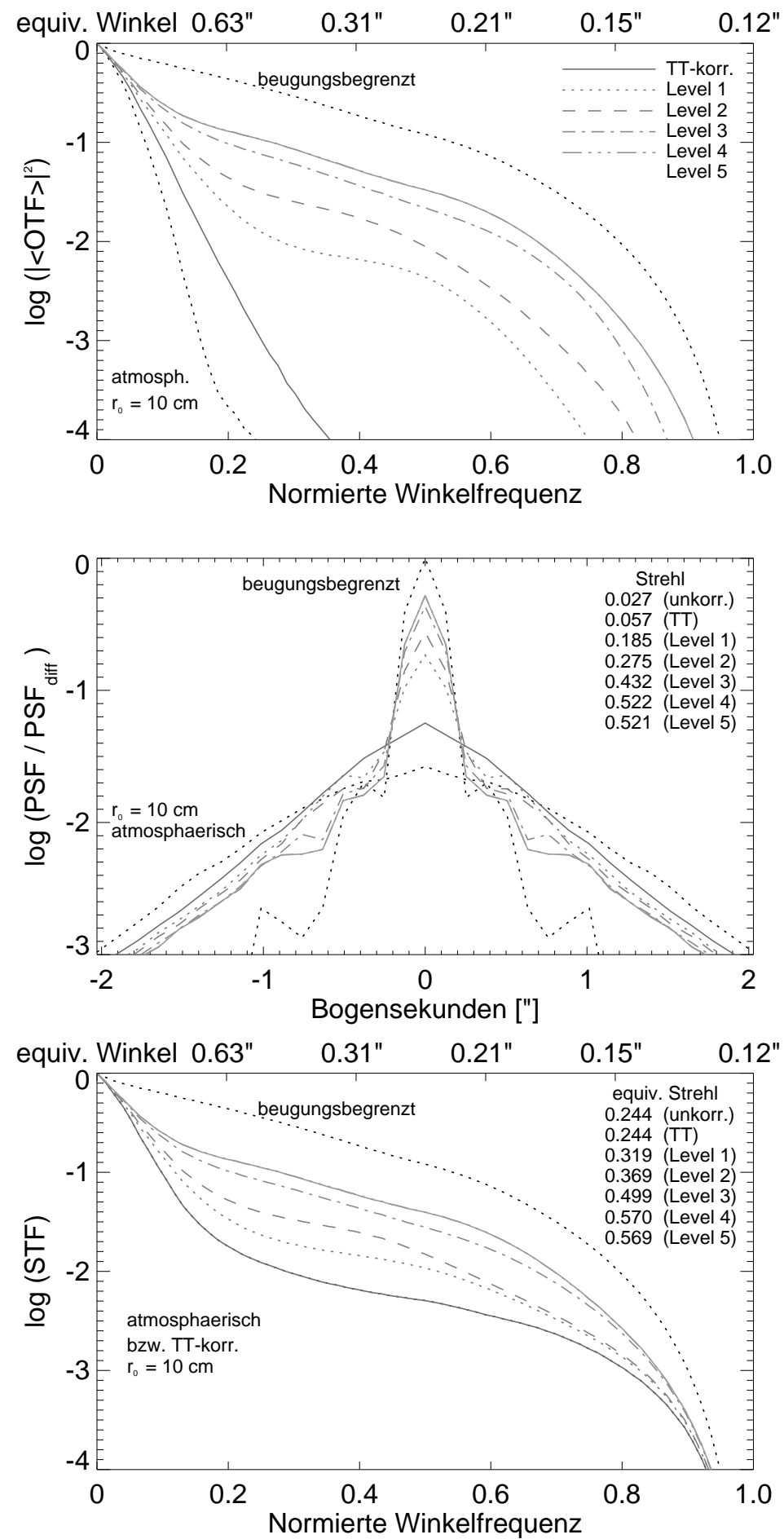

Abbildung 7.1: Zur Abhängigkeit der Ausgabegrößen der Simulation vom Korrekturlevel der Adaptiven Optik. Dargestellt sind von oben nach unten: Langzeit-Übertragungsfunktion, zugehörige PSF und STF (mittlere KurzzeitÜbertragungsfunktion). Die Rechnungen gelten am Lockpunkt und sind für mittlere Seeing-Bedingungen $\left(r_{0, \text { eff }}=10 \mathrm{~cm}\right)$ berechnet. 
sationsstärke der AO. Wie in Abschnitt 5.3.3 beschrieben wurde, unterschieden wir fünf Stufen der Empfindlichkeit bei der Berechnung der Aktuatorsignale für den DM. Hinzu kommt die Kompensation der Bildunruhe mit dem TT-Spiegel und wir zählen somit sieben Grade der Korrekturstärke, wenn der unkorrigierte Fall miteinbezogen wird.

Zunächst betrachten wir jedoch die in der Simulation berechneten mittleren Größen, also Langzeit-OTF, Langzeit-PSF und STF. Dabei beschränken wir uns in der Darstellung auf die azimutal gemittelte, eindimensionale Form, um die Abhängigkeit von den Parametern besser sichtbar zu machen. In Abbildung 7.1 ist zunächst ein Beispiel für die Bedeutung des Korrekturniveaus gezeigt. Der effektive Fried-Parameter von $r_{0}=10 \mathrm{~cm}$ bedeutet recht annehmbare Seeing-Bedingungen für Aufnahmen von Speckle-Serien. In der Abbildung erkennt man dies im untersten Feld, wo die STF für diesen Fall gezeigt wird. Schon ohne irgendeine Korrektur sieht man das Plateau der Signalstärke bei einem für die Speckle-Rekonstruktion hohen Signal/Rauschen-Abstand. Erst ab einer relativen Stärke von etwa $10^{-4}$ und darunter führt das schwache Signal zu signifikanten Fehlern bei der Phasenrekonstruktion. Mit zunehmendem Korrekturniveau steigt das Signal je nach Frequenz bis auf ein Vielfaches des unkorrigierten Wertes, vergrößert also in der Folge die Zuverlässigkeit bei der Phasorenbestimmung im Rekonstruktionsalgorithmus. Die extreme Verbesserung der Lichtkonzentration in einen kleinen Raumwinkel wird jedoch in der Darstellung der PSFs am deutlichsten. Diese sind in der mittleren Graphik von Abb. 7.1 dargestellt. Während die Bildberuhigung das Strehl-Verhältnis schon verdoppelt und die Halbwertsbreite der PSF von 1".5 auf 0!.8 fast halbiert, bleibt die Form einer Gaußförmig Verbreiterung weitgehend erhalten. Dagegen bildet sich bei Einsatz des deformierbaren Spiegels schon bei geringster Empfindlichkeit, also etwa der Korrektur der Zernike-Moden zweiter Ordnung, ein beugungsbegrenzter Zentralbereich heraus, der sich über einem Halo erhebt, der durch die nicht-korrigierten atmosphärischen Wellenfrontfehler verursacht wird. Mit immer höherer Korrektur wird dieser Untergrund immer schwächer, wie es auch die Theorie vorhersagt, und wir haben es im präsentierten Fall mit quasi-beugungsbegrenzter Abbildung zu tun. Die zu den PSFs gehörenden LangzeitMittelwerte der OTFs, also die Darstellung des diskutierten Falles im Fourierraum, bringt prinzipiell nichts Neues, liefert aber eine zusätzliche Sichtweise auf die Korrekturverbesserung. So ist die Ausbildung des 'Buckels' oder Plateaus mit einsetzender DM-Korrektur ein Zeichen für das Erscheinen eines Airy-Scheibchens vor dem atmosphärisch veränderlichen Hintergrund.

Werfen wir nun einen Blick auf die Abhängigkeit von der Turbulenzstärke in der Atmosphäre. Ähnlich wie in Abb. 7.1 sind in den Abbildungen 7.2 und 7.3 auf den folgenden beiden Seiten für gewisse Kombinationen aus den beiden Fried-Parametern der Turbulenzschichten jeweils die oben besprochene Gruppe der drei errechneten Funktionen, OTF, PSF und STF, aufgetragen. Gerade für Anwendungen, die lange Belichtungszeiten diktieren, wie etwa Beobachtungen in spektral sehr schmalbandigen Durchlassbereichen, zeigt die Adaptive Optik ihren größten Nutzen, wie vor allem die Graphen der LangzeitOTF erkennen lassen. Vergleicht man die Kurven für verschiedenes $r_{0 \text {,unten }}$ in Abb. 7.2, so wird deutlich, dass die Korrekturleistung selbst von besseren Ausgangsbedingungen in der Atmosphäre profitiert bzw. bei schlechteren darunter leidet. Es ist also daraus ersichtlich, dass ein guter Beobachtungsstandort auch durch die technische Hilfe einer Adaptiven Optik nicht verzichtbar werden wird. Für die Speckle-Anwendung hat der Gewinn durch die Adaptive Optik zum einen den Aspekt der größeren Effizienz, da zur Phasen- 

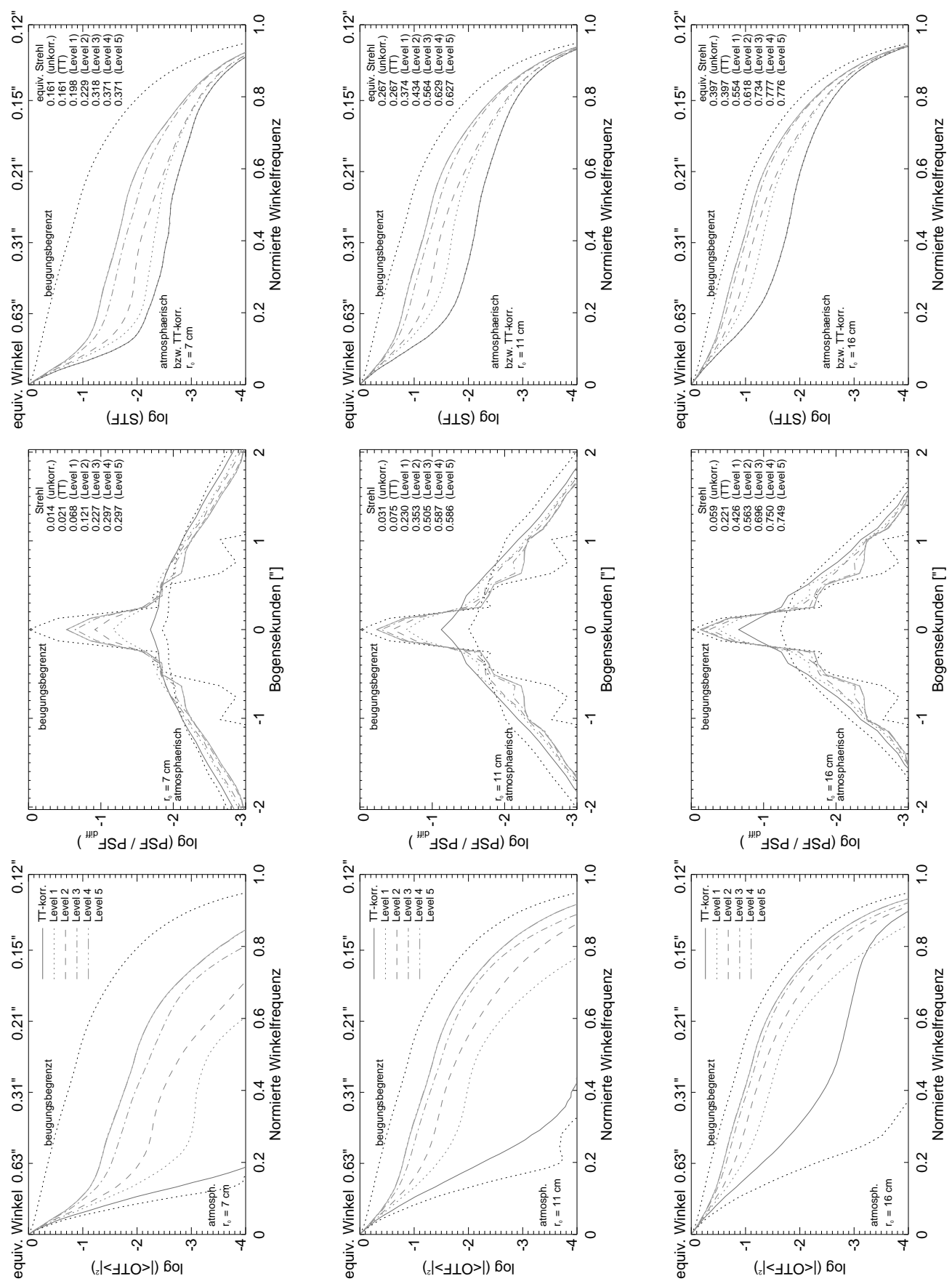

Abbildung 7.2: Zur Abhängigkeit der AO-Kompensationleistung am Lockpunkt von Korrekturgrad und der Turbulenzstärke bodennaher Luftschichten. Nach Drehung der Abbildung um $90^{\circ}$ gilt: Von oben nach unten verbessern sich die atmosphärischen Bedingungen mit $r_{0 \text {,unten }}=7,12$ und $20 \mathrm{~cm}$. Der Fried-Parameter $r_{0, \text { oben }}$ der oberen Turbulenzschicht beträgt konstant $30 \mathrm{~cm}$. In jedem Feld sind Kurven für alle Korrekturlevel gezeigt. Dabei gilt die Linienlegende der linken Spalte auch für die übrigen Felder. Die linke Spalte der Graphiken zeigt die Langzeit-OTFs, die mittlere die zugehörigen PSFs und die rechte die entsprechenden STFs. 

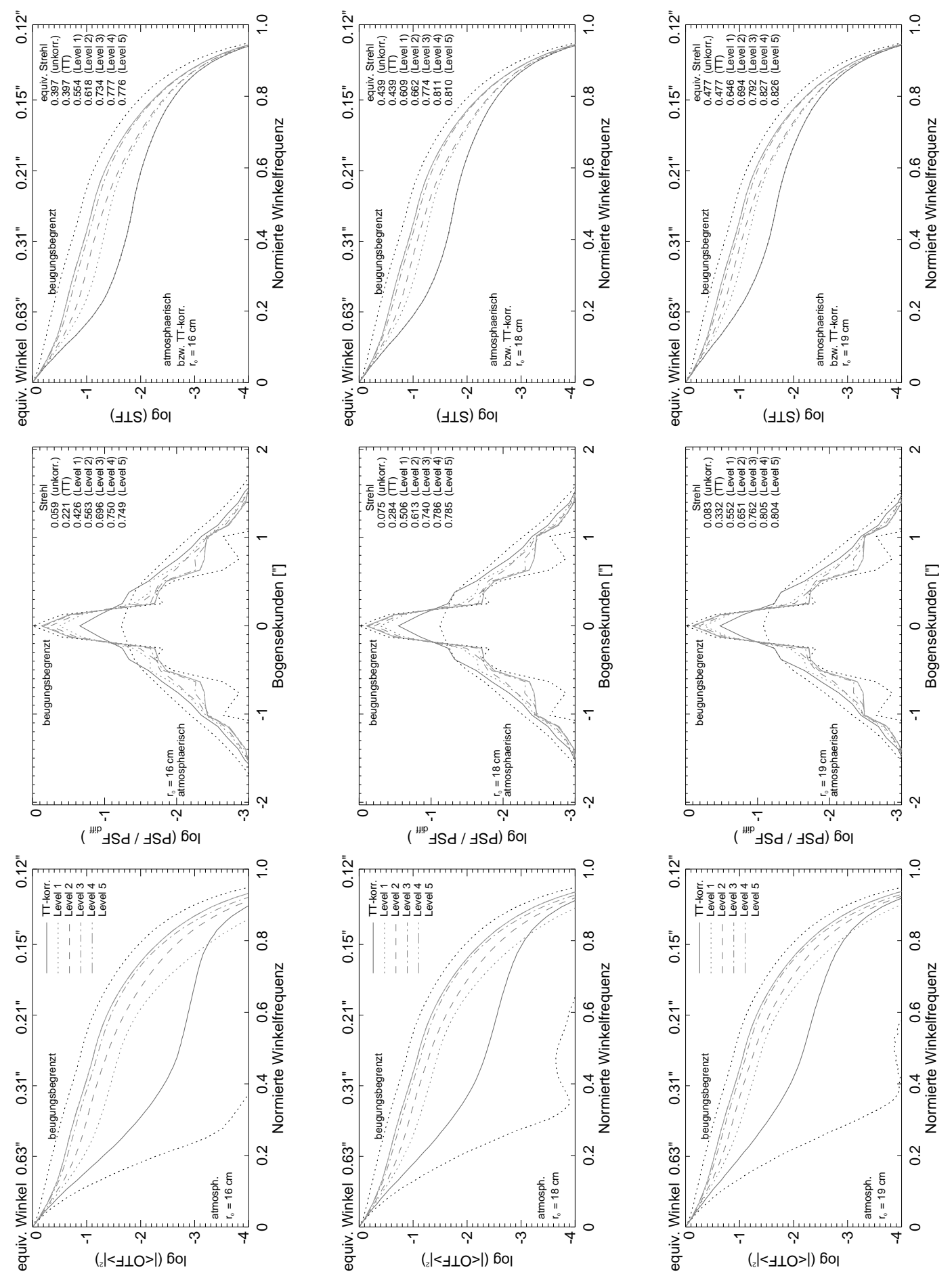

Abbildung 7.3: Zur Abhängigkeit der AO-Kompensationleistung von der Turbulenzstärke der oberen Atmosphärenschicht. Anordnung und Legende wie in Abb. 7.2. Nach Drehung der Abbildung um $90^{\circ}$ gilt: Von oben nach unten verbessert sich $r_{0, \text { oben }}$ (30, 50 und $100 \mathrm{~cm}$ ). Der Fried-Parameter $r_{0, \text { unten }}$ der unteren Turbulenzschicht beträgt hier konstant $20 \mathrm{~cm}$. 
bestimmung wegen des besseren SNR weniger Mittelungen erforderlich sind und früher abgebrochen werden können. Andererseits bleibt die Amplitudenrekonstruktion empfindlich gegenüber der Signalstärke, wenn auf photometrische Genauigkeit geachtet werden soll. Im Fourierraum wird ja durch eine Multiplikation mit dem Kehrwert der STF bei jeder Frequenz der effektive Informationsverlust durch die Atmosphäre nach AO-Korrektur, zusammen mit der Signalverminderung durch die Beugung an der Teleskopöffnung, ausgeglichen. Da durch die AO das Signal, abhängig von der Frequenz, oft um ein Vielfaches geändert wird, ist auch hier im Bereich der kurzen Belichtungszeiten eine genaue Modellierung nötig.

Die Abhängigkeit von der oberen Turbulenzschicht ist bei den untersuchten Turbulenzstärken von $30 \mathrm{~cm}$ bis $100 \mathrm{~cm}$ (siehe Abb.7.3) wenig ausgeprägt. Da in der Simulation die Phasenschirme beider Schichten nur addiert werden, bestimmt bei solchen Größenunterschieden der Fried-Parameter natürlich der Phasenschirm mit den größeren Fluktuationen die Ausgangssituation und damit das Korrekturvermögen der AO.

Die Strehl-Zahlen erlauben nun eine kompaktere Darstellung der Abbildungsqualität und der Verbesserungen durch die AO-Korrektur. In Abbildung 7.4 sind für die Fälle einer Langzeitbelichtung (links) und einer Kurzzeitbelichtung (rechts) Kurven für die steigenden Strehl-Zahlen über dem Korrekturniveau aufgetragen. Für drei Werte des FriedParameters der oberen Turbulenzschicht bezeichnen jeweils innerhalb eines Feldes verschiedene Kurven verschiedene Turbulenzstärken der teleskopnahen Luftmassen. Es lässt sich eine gewaltige Verbesserung der Bildqualität durch die Wellenfrontkorrekturen ablesen! Schon die einfache Stabilisierung des Bildes bringt bekanntlich eine oft entscheidende Verbesserung, da 4/5 der Varianz der atmosphärisch verzerrten WF auf die zugehörigen Zernike-Moden (Tip/Tilt) entfallen. Zum weiteren Verlauf der Kurven bedenke man, dass für die dargestellten Fälle (große Strehl-Zahlen) das Maréchal'sche Kriterium gilt (Gl. 3.31) und somit Rückschluss auf die Varianz $\sigma^{2}$ des residuellen Wellenfrontfehlers über $\sigma^{2}=-\ln ($ Strehl $)$ erlaubt. Wir haben es also bei bestem Korrekturgrad für die dargestellten Seeing-Bedingungen fast durchweg mit beugungsbegrenzter Abbildung zu tun. Der fast lineare Anstieg geht dann bei der empfindlichsten Einstellung der Adaptiven Optik in eine Sättigung. Hier ist der begrenzende Faktor die Anzahl der korrigierbaren Freiheitsgrade, also gewissermaßen die Anzahl von Subaperturen und Aktuatoren. Für die Korrekturniveaus 4 und 5 sind alle 18 Freiheisgrade des modellierten Systems aktiv. Zusätzlich fällt wie bei den Betrachtungen zu Abb. 7.2 auf, dass der Verbesserungsfaktor für alle betrachteten Turbulenzstärken etwa gleich ist und somit auch bessere Ergebnisse erzielt werden können wenn auch die Ausgangsbedingungen der Atmosphäre gut sind. Ebenfalls ist zu erkennen, dass die Effizienz des Systems in Einklang mit der Theorie etwa bei einer effektiven Turbulenzstärke von $10 \mathrm{~cm}$ liegt.

Im Falle einer Kurzzeitbelichtung (rechte Hälfte der Abbildung) startet man einerseits von besseren Werten, andererseits findet eine Bildstabilisierung keine Anwendung, da die kurze Belichtungszeit allein schon eine Methode zur Beseitigung von Verwackelungsunschärfen darstellt. Insgesamt findet man jedoch ein ähnliches Verhalten wie bei der Langzeit-Mittelung, von der kleineren Magnitude der relativen Verbesserungen abgesehen. Die Strehl-Zahlen im bestkorrigierten Fall sind in etwa gleich den Werten für die Langzeitbelichtung.

Lässt man nun die Werte für die Turbulenzstärke der oberen Schicht variieren und hält dabei den Fried-Parameter der teleskopnahen Schicht konstant, präsentiert sich am Lock- 

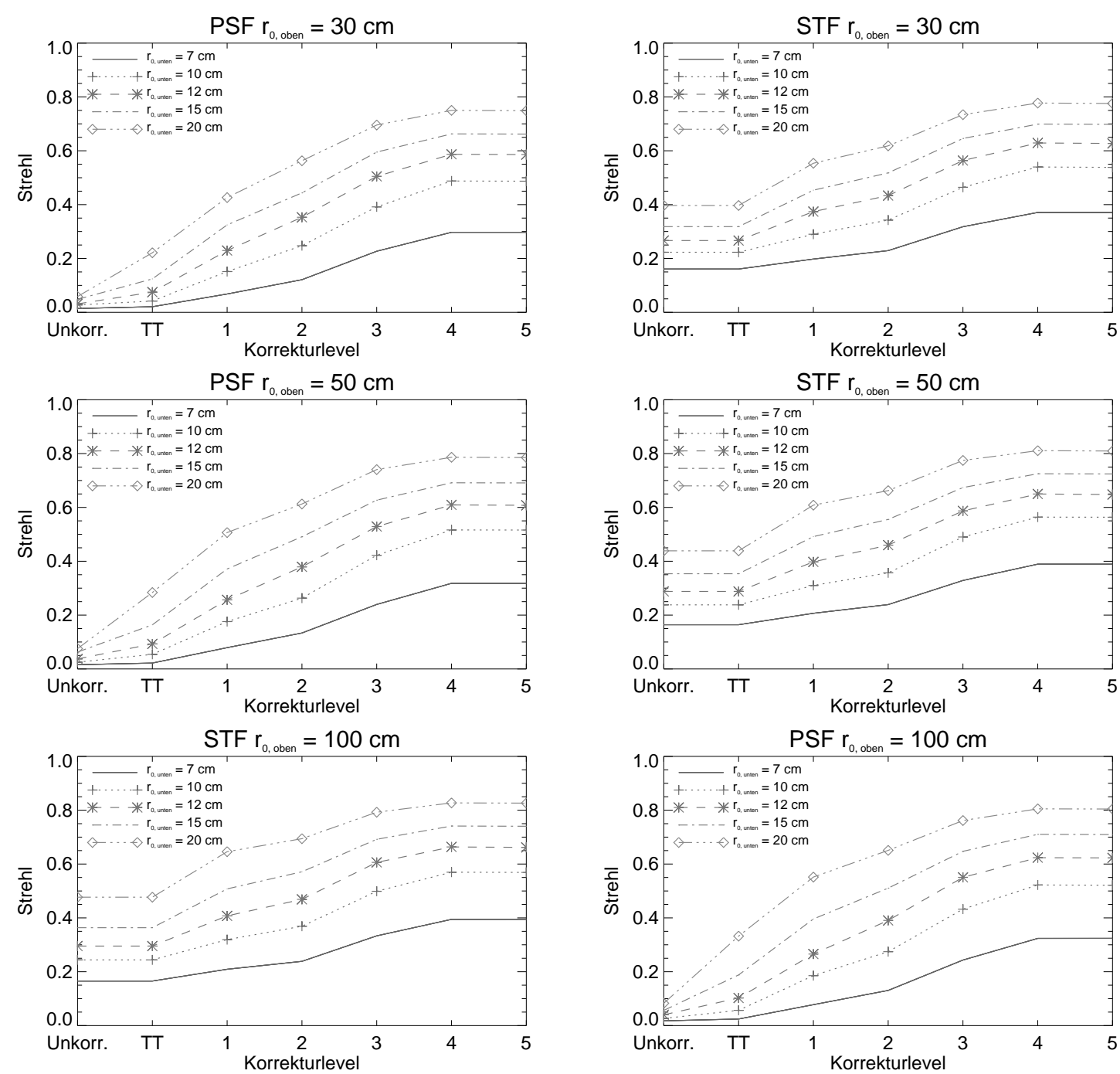

Abbildung 7.4: Verbesserung der Abbildungsleistung anhand des Strehl-Verhältnisses bei Langzeitbelichtungen (linke Spalte) und Kurzzeitbelichtungen (rechte Spalte) in Abhängigkeit vom Korrekturlevel der Adaptiven Optik. Die dargestellten Kurven bezeichnen verschiedene Turbulenzstärken der teleskopnahen Turbulenzschicht, jedoch bei festgehaltenem Wert $r_{0, \text { oben }}$ für die hohe Atmosphäre. Die Rechnungen gelten für den Lockpunkt.

punkt eine sehr viel schwächere Abhängigkeit der Bildqualität von diesem Parameter, also von den Bedingungen der oberen Atmosphäre. Für drei Turbulenzstärken am Teleskop ist diese Situation in Abbildung 7.5 zusammengefasst, wobei wie in den Betrachtungen zuvor das erreichte 'Strehl' gegen das eingesetzte Korrekturniveau aufgetragen ist: Sowohl im Falle einer Langzeitbelichtung (linke Spalte der Abb.) wie auch bei den Kurzzeitbelichtungen (rechte Spalte der Abb.) macht sich die obere Atmosphärenschicht praktisch nur bei sehr gutem bodennahen Seeing bemerkbar. Je besser die Korrektur wird, desto kleiner werden jedoch diese Unterschiede wieder. Im Hinblick auf die reale Beobachtungssituation, wo der Regelkreis der Adaptiven Optik immer die bestmögliche Korrekturmatrix bei gegebenen Seeing-Bedingungen zu verwenden sucht, ist also bei 'normalen' atmosphäri- 

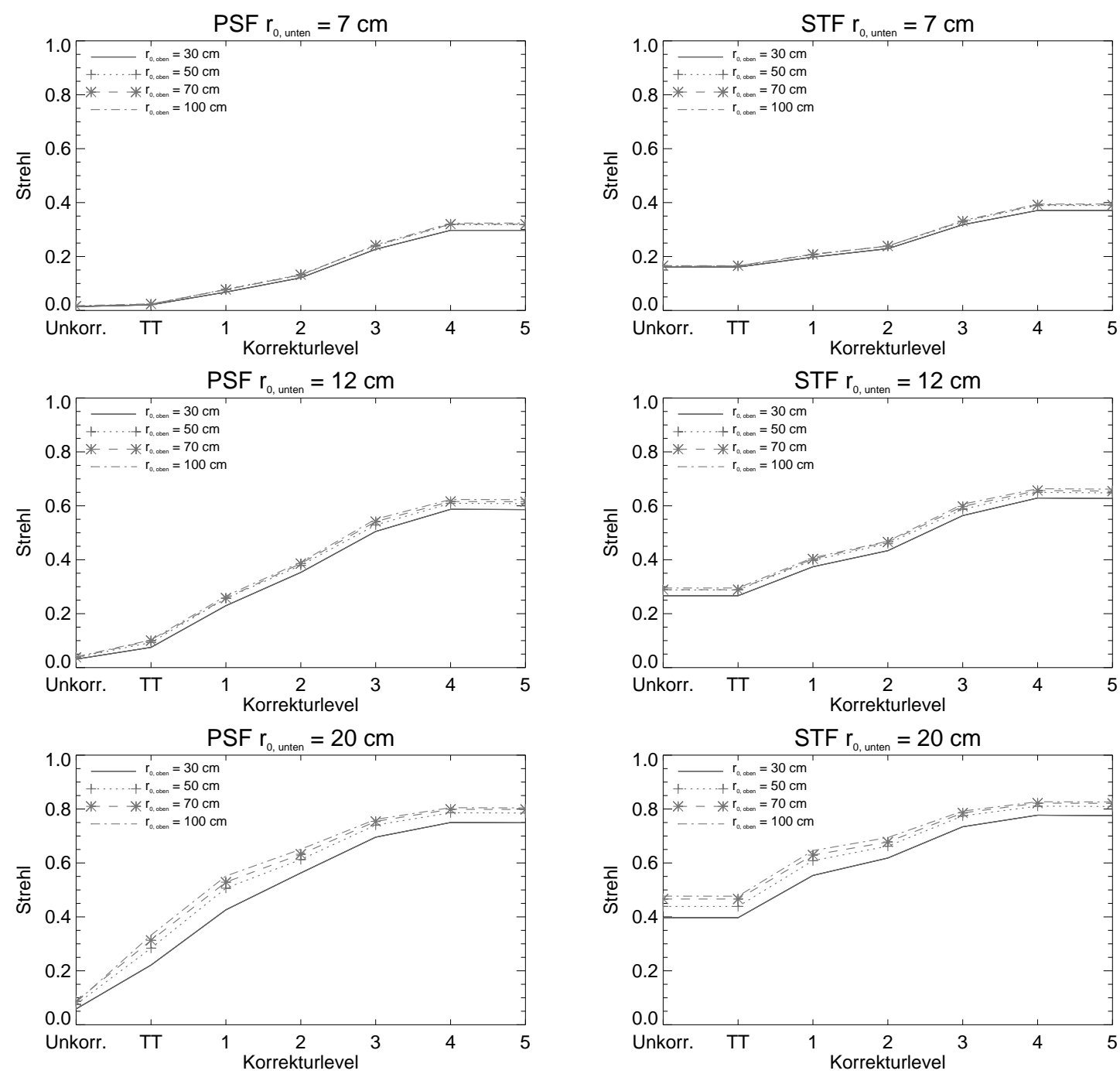

Abbildung 7.5: Abhängigkeit vom Korrekturlevel bei konstanter teleskopnaher Turbulenzstärke für die Werte $7 \mathrm{~cm}, 12 \mathrm{~cm}$ und $20 \mathrm{~cm}$ für $r_{0}$,unten, von oben nach unten ansteigend. Wie in Abbildung 7.4 dient das mit dem Korrekturgrad steigende Strehl-Verhältnis als Maß für die Verbesserung der Bildqualität. Die linke Spalte der Graphen gilt für Langzeitbelichtungen, die rechte Spalte beschreibt die durchschnittliche Kurzzeitbelichtung. Interpretation siehe Text.

schen Bedingungen die ausschlaggebende Größe die bodennahe Turbulenz. Herrscht dort schlechtes Seeing, kann die Wellenfront nicht ausreichend genau abgetastet werden und das System reagiert mit Verringerung der Empfindlichkeit. Somit fällt auch das Korrekturniveau niedrig aus. Wirft man einen Blick auf die Graphen der oberen Reihe von Abb. 7.5, erkennt man keinen bedeutsamen Unterschied für variable Bedingungen der oberen Atmosphäre. Am anderen Ende dieser Skala liefert der WFS bei niedriger Turbulenzstärke am Teleskop ein optimales, rauscharmes Signal und die größte Empfindlichkeit des Systems kann und wird eingestellt werden. Die entsprechenden Graphen in der unteren Zeile der besprochenen Abbildung zeigen auch für diesen Fall nur eine sehr geringe Abhängig- 
keit von der schwächeren oberen Turbulenzschicht.

Insgesamt lässt sich dieser Effekt anhand der Zahlenwerte für das Seeing beider Schichten schon ablesen, denn in der Simulation werden Phasenschirme, wie sie nach Durchlaufen jeder Schicht allein resultieren, einfach addiert. Somit hat auch die Schicht mit dem kleineren Fried-Parameter, also größeren induzierten Weglängenunterschieden, das größere Gewicht und gibt den Ausschlag für die effektive WF-Deformation. Es lässt sich allerdings auch in Abb. 7.5 schon erkennen, dass die Unterschiede in den Kurven im Falle von $r_{0, \text { unten }}=20 \mathrm{~cm}$ am unteren Ende der Werteskala größer werden. Hier nähern sich die Seeing-Bedingungen beider Schichten aneinander an und werden in ihrer Größe vergleichbar. Findet man also die Situation starker Turbulenz an der oberen Grenze der Troposphäre vor, wie etwa im Falle des 'Jet-Streams', kann sich das Verhältnis der Gewichte gar umkehren, sodass die obere Turbulenzschicht hochauflösende Beobachtungen durch zusätzlich hohe Windgeschwindigkeiten bisweilen ganz verhindert.

\subsubsection{Winkelabhängigkeit}

Nachdem im vorigen Abschnitt alle Modellrechnungen für den Lockpunkt der AO galten, erweitern wir jetzt unseren Blickwinkel und betrachten den Einfluss der Wellenfrontkorrektur auf die Abbildung von Strukturen, die im Bildfeld abseits der für den WFS sichtbaren Lockpunktregion liegen. Dabei sei nocheinmal daran erinnert, dass sich alle Simulationsrechnungen in einem Modell von zwei Turbulenzschichten in der Atmosphäre bewegen, wobei die untere, teleskopnahe Schicht auf alle Blickrichtungen dieselbe Wirkung zeigt und nur die hohe Atmosphärenschicht beim Blick in verschiedene Richtungen ein unterschiedliches Bild liefert (vgl. dazu Abb. 3.4). Als Höhe der Schicht wurden $8000 \mathrm{~m}$ festgesetzt, sodass für eine Blickrichtung 18" abseits des Lockpunktes der Ausschnitt der Wellenfront in der oberen Turbulenzschicht, der dann von unserem $70 \mathrm{~cm}$-Teleskop 'eingefangen' werden wird, gerade vollständig neben dem entsprechenden WF-Ausschnitt der Lockpunktrichtung liegt ( $\Delta=1$ in Abb. 3.5). In der Simulation wurden ähnliche Kurven berechnet wie die im vorigen Abschnitt besprochenen, nun jedoch mit einem zusätzlichen Freiheitgrad, der Blickrichtung. Dabei wurde letztere für Winkelabstände zwischen $0^{\prime \prime}$ und $30^{\prime \prime}$ in Schritten von etwa $3^{\prime \prime}$ berechnet.

In Abbildung 7.6 sehen wir wieder nebeneinander Langzeit-OTF, Langzeit-PSF und STF, wobei die gemittelte OTF zum besseren Vergleich mit der STF hier wieder als quadrierte Größe dargestellt wird. In jedem Fenster sind nun die Funktionen für die verschiedenen Blickrichtungen aufgetragen. Von oben nach unten steigt das Korrekturlevel von unkorrigiert über schwache DM-Korrektur (AO-Level 2) zum bestmöglich korrigierten Fall (AOLevel 5). Die atmosphärischen Parameter sind konstant gehalten, wobei die obere Turbulenzschicht großen Einfluss besitzt $\left(r_{0, \text { unten }}=10 \mathrm{~cm}\right.$ und $\left.r_{0, \text { oben }}=30 \mathrm{~cm}\right)$. Im Vergleich mit den Graphen aus Abbildung 7.3 bemerkt man an den OTF-Kurven eine qualitativ andere Abhängigkeit des Frequenzverlaufs: Hatten wir bei der Abhängigkeit vom Korrekturlevel vorwiegend die abrupte Ausbildung eines flachen Plateaus der Graphen bis zu hohen Winkelfrequenzen und seine Anhebung beobachtet, wie es für die Form der STF typisch ist, so findet die Abschwächung des Signals, von dieser Augangssituation, bei größer werdendem Blickwinkel eher gleichmäßig auf allen Frequenzen statt. Im oberen Feld ist vom AO-System erst der Kipp-Spiegel (TT) eingeschaltet und es sind die statistischen Schwankungen in den Mittelwerten der höheren Moden zu erkennen, die bei 

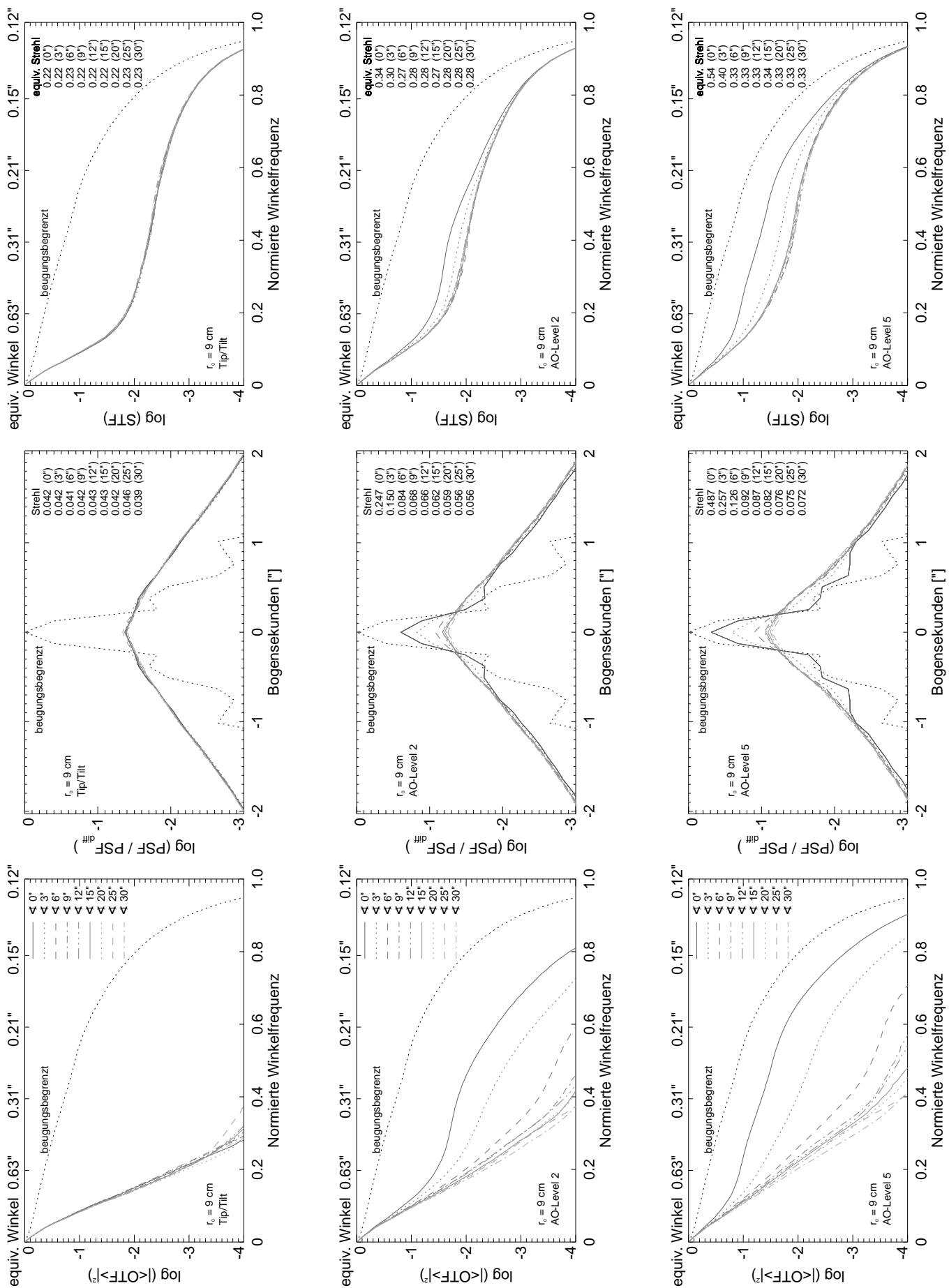

Abbildung 7.6: Zur Winkelabhängigkeit der AO-Kompensation. In jedem Feld sind Graphen für verschiedene Blickwinkel relativ zur Lockpunktrichtung gezeigt (Legende in den Fenstern der linken Spalte). Die Anordnung der Funktionstypen entspricht der in Abbildung 7.2. Nach Drehung der Abbildung um $90^{\circ}$ gilt: Von oben nach unten verbessert sich die WF-Korrektur, bei den festen Fried-Parametern $r_{0 \text {,unten }}=10 \mathrm{~cm}$ und $r_{0, \text { oben }}=30 \mathrm{~cm}$. 

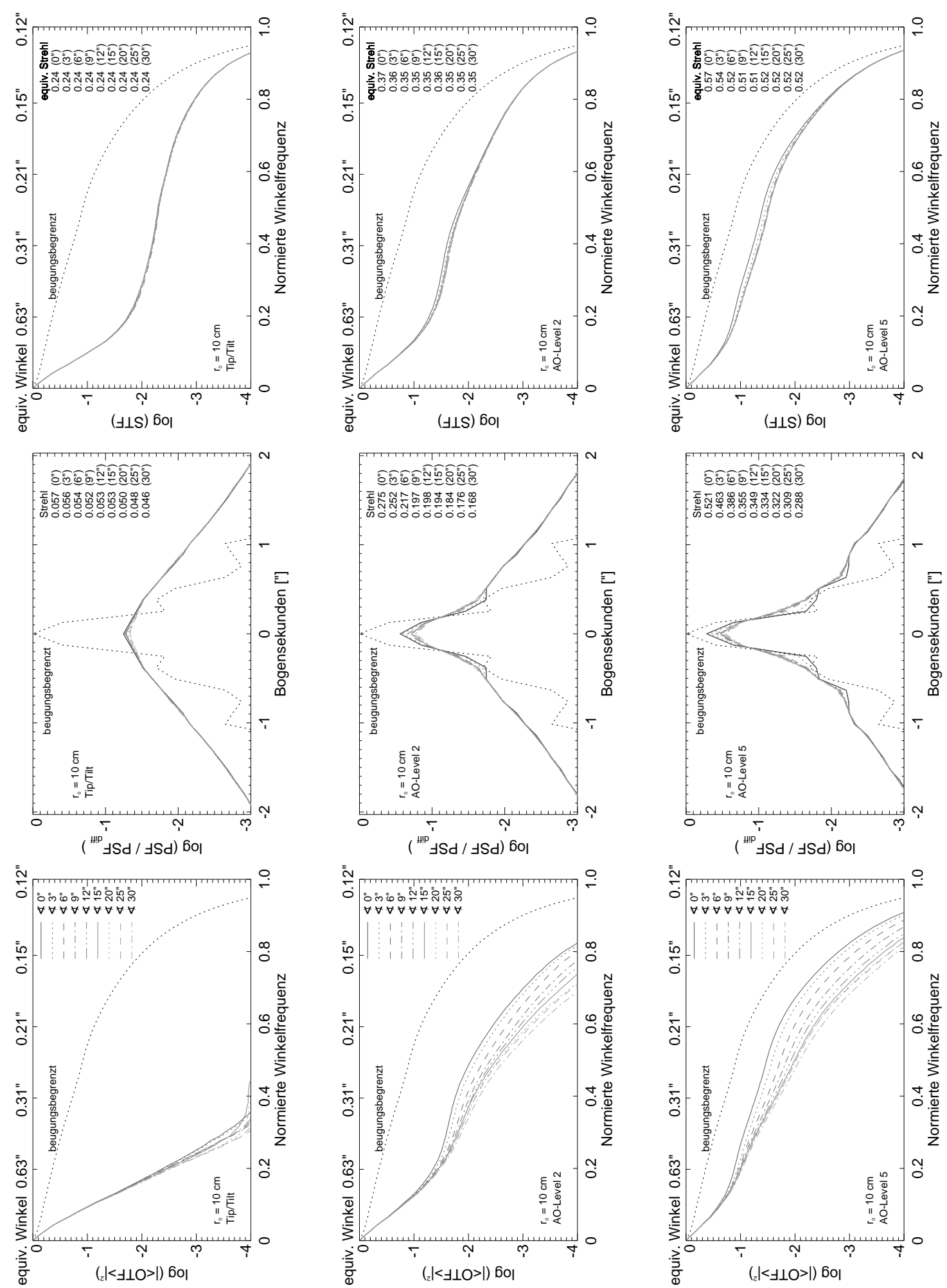

Abbildung 7.7: Zur Winkelabhängigkeit der AO-Kompensation. Die modellierte Situation entspricht der in Abb. 7.6 beschriebenen. Der Fried-Parameter der unteren Schicht ist $r_{0, \text { unten }}=10 \mathrm{~cm}$, der der oberen Turbulenzschicht beträgt hier jedoch $r_{0, \text { oben }}=100 \mathrm{~cm}$. 
der Berechnung der Funktionen aus jeweils hundert etwas gegeneinander verschobenen WF-Ausschnitten auftreten. Bei einsetzender Korrektur bildet sich das schon diskutierte Plateau bei der zum Lockpunkt gehörenden Kurve aus, das aber auch bei voller Korrektur mit wachsendem Winkel rasch abfällt. Ab einem Winkel von etwa 10" stagniert die Langzeit-OTF in einem Zustand, dessen Graph jedoch eine deutlich schwächere Steigung aufweist, also das Signal bis in den mittleren Frequenzbereich hinein auf ein deutlich höheres Niveau hebt, als nur mit Bildstabilisierung möglich war. Die zugehörige räumliche Skala, die dadurch bei den in Betracht gezogenen Winkelabständen zugänglich, d. h. aufgelöst wird, ist am besten in den mittleren Darstellungen der PSFs an der Halbwertsbreite abzulesen, die im TT-korrigierten Fall 1".2 beträgt, und bei AO-Level 5 auf 0 ".8 absinkt. Im Falle der STF teilt sich die Verbesserung des Signals durch die AO in zwei Effekte auf. Der eine Effekt besteht in einer Art 'Glättung' des Bereichs der Kurven, wo der Graph sich zum Plateau hin krümmt. Diese Anhebung des Signals wirkt auf alle Kurven im gleichen Maße, hat also eine höchstens sehr schwache Winkelabhängigkeit. Dagegen weist der zweite Anteil der Korrektur eine starke Abhängigkeit auf (vgl. Fusco 2004). Er ist auf einen kleinen Winkel von etwa $5^{\prime \prime}$ um den Lockpunkt herum konzentriert und verursacht die sichtbare Auffächerung der Kurvengruppe. Diese Anhebung wird durch die Korrektur hoher Ordnung verursacht und reicht bis zu den zugehörigen räumlichen Frequenzen, die mit der Korrekturstärke anwachsen. Daran kann beispielsweise auch abgelesen werden, dass etwa das AO-Level 2 bis zu einer Skala von knapp unter 0".3 die stärksten Korrektureffekte zeigt, dagegen können bei AO-Level 5 Strukturgrößen von 0 '”2 korrigiert werden.

Wenden wir uns der Abbildung 7.7 zu. Die dargestellte Situation ist bis auf die viel schwächere obere Turbulenzstärke $\left(r_{0, \text { oben }}=100 \mathrm{~cm}\right)$ identisch zu der in Abb. 7.6. Alle dazu angestellten Beobachtungen und Überlegungen gelten auch hier, mit dem augenscheinlichen Unterschied der bedeutend schwächeren Winkelabhängigkeit. Sehr deutlich sind in den Graphen der STF und hier auch in denen der Langzeit-OTF die 'Buckel' der vorzugsweise korrigierten räumlichen Skalen zu sehen. Die AO-korrigierten PSFs besitzen allesamt einen stark ausgeprägten beugungsbegrenzten Kern und einen sehr geringen Streulichtuntergrund. Solche Bilder eignen sich hervorragend zur Weiterverwendung in Entfaltungsalgorithmen.

Zur Zusammenfassung der Winkelabhängigkeit sind in Abbildung 7.8 die Strehl-Zahlen für Kombinationen der atmosphärischen Turbulenzstärken $r_{0, \text { unten }}$ und $r_{0, \text { oben }}$ gegen die Winkel im Gesichtsfeld zusammengestellt. Dabei ist hier die volle AO-Kompensation angenommen und wir unterscheiden wieder die normalen Strehl-Zahlen für die Langzeitbelichtungen mit der Marke 'PSF' und die äquivalenten Strehl-Zahlen, wie sie aus den STFs folgen. Auffällig hierbei ist wieder der kleine Winkel um den Lockpunkt, in dem die Korrektur hoher Ordnungen stattfindet. Dieser Bereich ändert sich in seiner Größe nicht sehr deutlich abhängig von der Turbulenzstärke der oberen Schicht, was zu erwarten gewesen wäre. Allerdings liegen die 'Sättigungsniveaus' mit kleiner werdendem $r_{0, \text { oben }}$ höher und führen, bei guten lokalen Seeing-Bedingungen um das Teleskop, tendenziell zu einem weit in die Außenbereiche eines Bildes reichenden Abfall der Strehl-Zahlen auf hohem Niveau. 

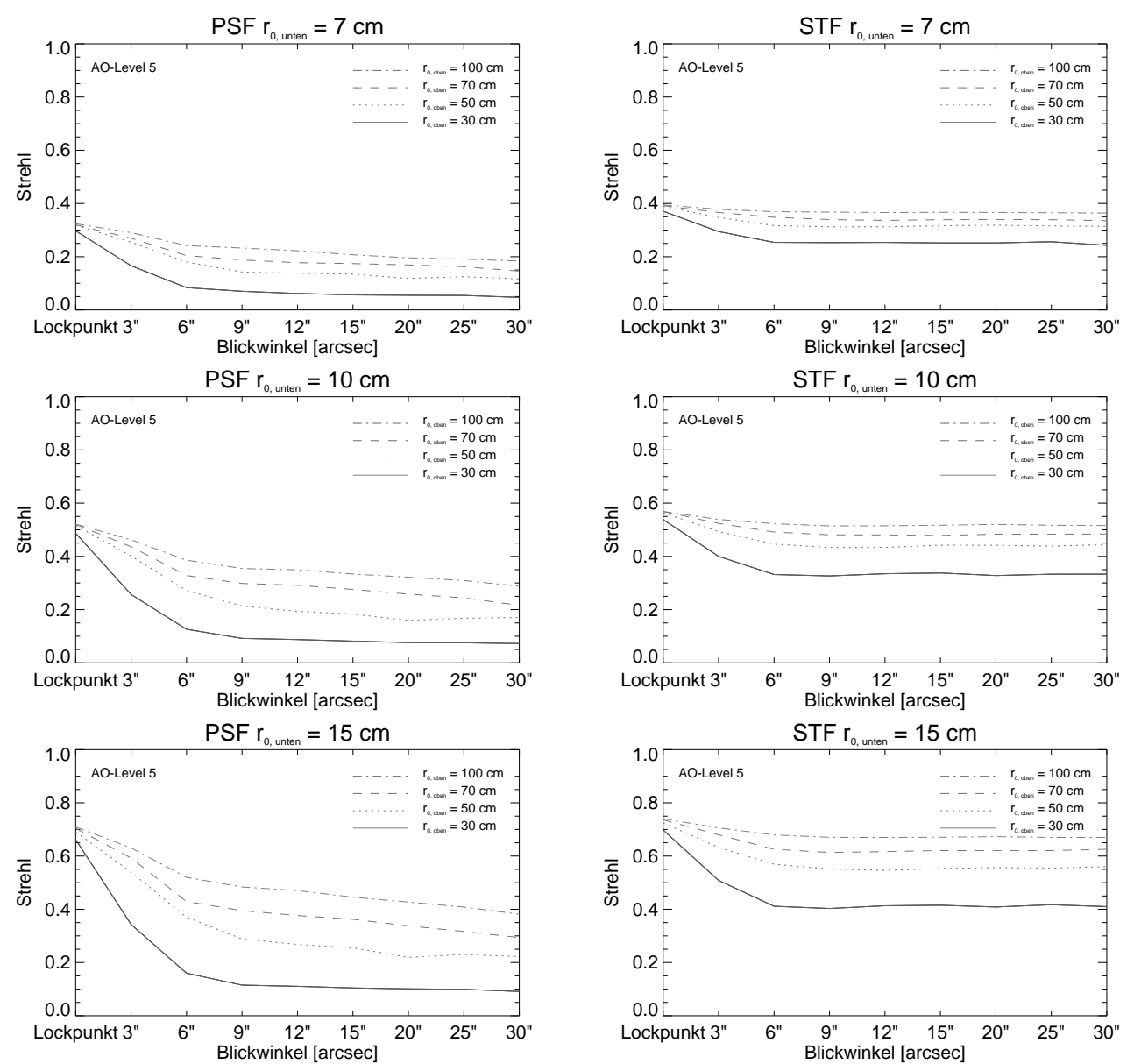

Abbildung 7.8: Für beste AO-Korrektur berechnete Strehl-Zahlen in Abhängigkeit von der Blickrichtung relativ zur Lockpunktrichtung. Links sind jeweils die aus den LangzeitPSFs berechneten Größen gezeigt ('PSF'), rechts die Strehl-Zahlen aus den STFs. Von oben nach unten nimmt der Fried-Parameter $r_{0 \text {,unten }}$ der teleskopnahen Schicht zu, innerhalb jedes Feldes gilt die Legende für die angenommene Turbulenzstärke in der oberen Atmosphäre.

\subsubsection{Dekorrelation durch Wind}

Wind spielt als Parameter für die Leistung einer Adaptiven Optik ebenfalls eine wichtige Rolle. Der uns interessierende Effekt besteht darin, dass Wind die Turbulenzzellen mit einer Geschwindigkeit $\boldsymbol{v}$ lateral zum Sichtstrahl mit sich trägt. Dazu bedenke man, dass immer eine Zeitdifferenz $\Delta t$ zwischen dem Zeitpunkt der WF-Messung und dem Zeitpunkt liegt, an dem der DM seine korrigierende Form tatsächlich angenommen hat. In der Folge wird die Korrektur immer auf eine um $\Delta \boldsymbol{x}=\boldsymbol{v} \cdot \Delta t$ verschobene WF angewandt. Durch den Versatz dekorrelieren die Flächen des DM und der tatsächlichen WF in Abhängigkeit von der zeitlichen Verzögerung und der Windgeschwindigkeit. In der Simulation wird nun der Versatz $\Delta \boldsymbol{x}$ variiert und die Kurven sind somit für alle Kombinationen von $v$ und $t$ gültig, deren Produkt diesen Wert liefert. Wir haben für die Legende der Graphiken angenommen, dass die zeitliche Verzögerung zwischen Messung der WF 

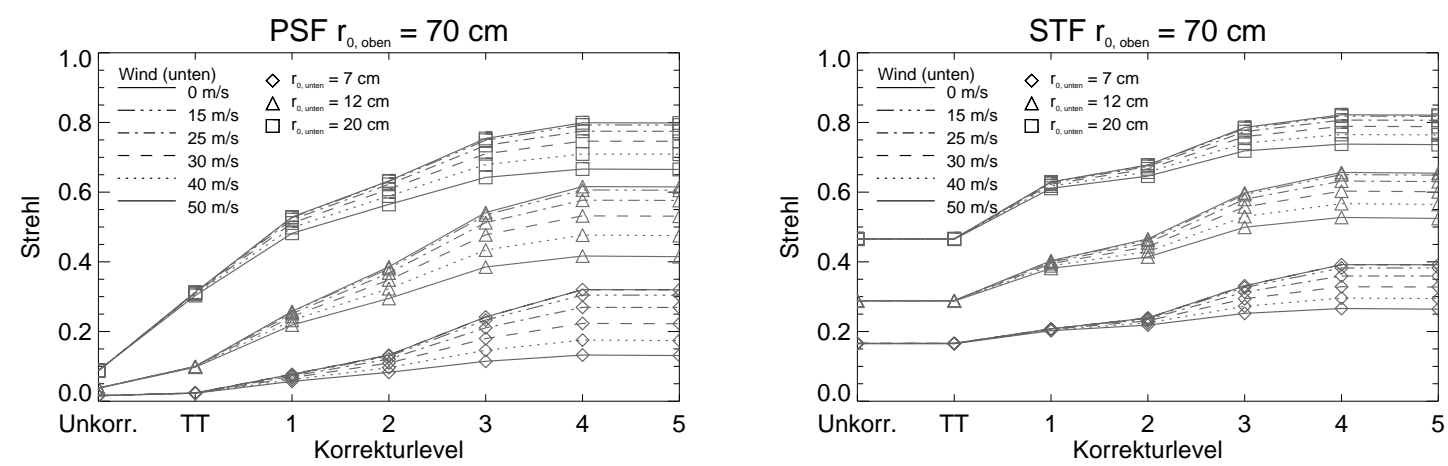

Abbildung 7.9: Strehl-Zahlen unter Einfluss von Wind am Teleskop im Falle einer Langzeitbelichtung (links) und einer mittleren Kurzzeitbelichtung (rechts): Für drei Werte des Fried-Parameters $r_{0 \text {,unten }}$ sind jeweils Kurven für die angegebenen Windstärken am Teleskop über zunehmender Korrekturstärke aufgetragen. Die Kurven sind dabei für den Lockpunkt berechnet und eine zeitliche Verzögerung von $1 \mathrm{~ms}$ wurde zur Umrechnung des Phasenschirm-Versatzes ( 0 bis $5 \mathrm{~cm}$ ) in die Windgeschwindigkeit angenommen.
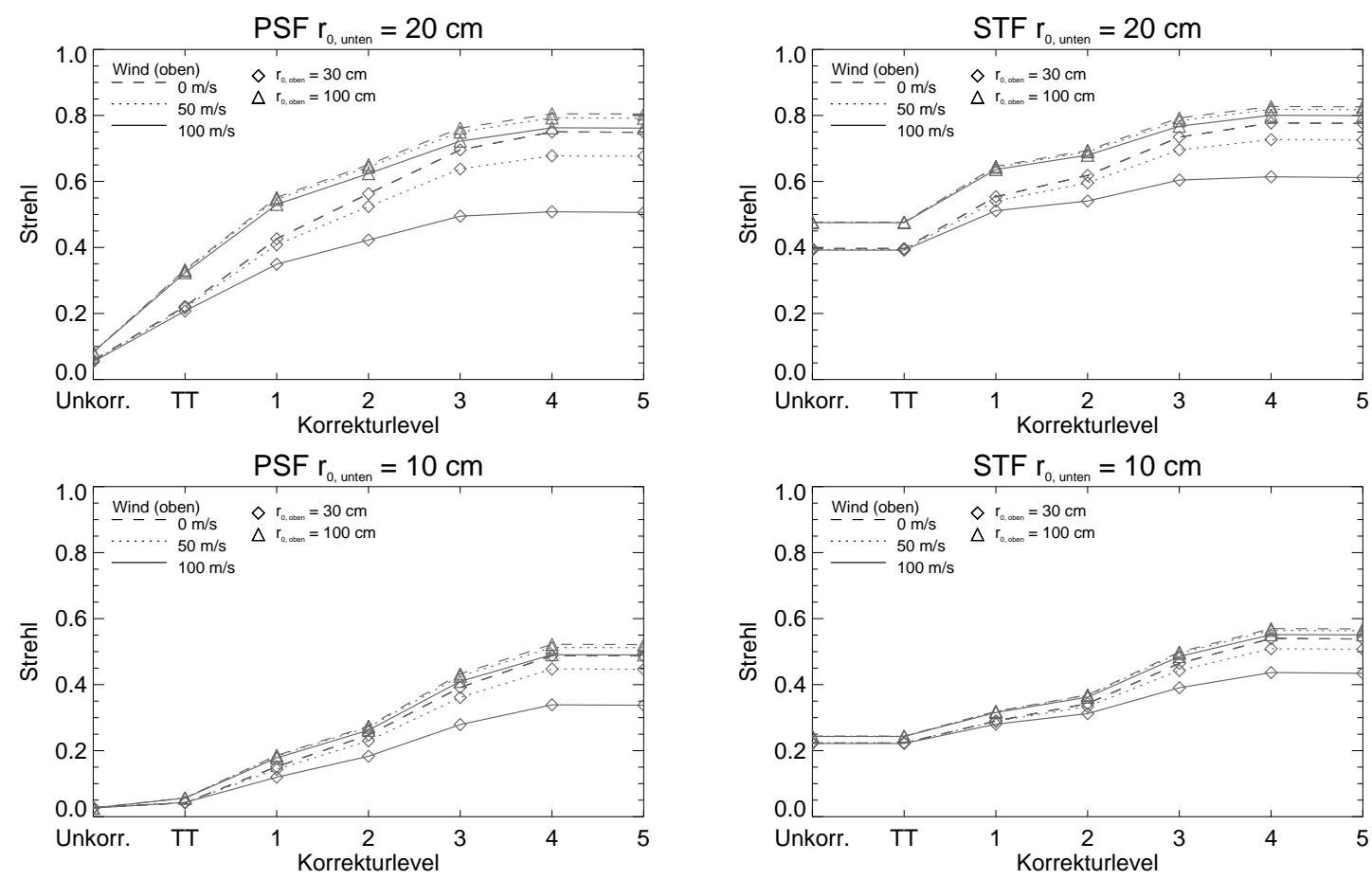

Abbildung 7.10: Strehl-Zahlen am Lockpunkt unter Einfluss von Wind in der oberen Turbulenzschicht, links bei Langzeitbelichtung, rechts für ein typisches Speckle-Bild. Oben: Hervorragende Seeing-Bedingungen am Teleskop $\left(r_{0, \text { unten }}=20 \mathrm{~cm}\right)$, unten: mittlere Turbulenzstärke $\left(r_{0 \text {,unten }}=10 \mathrm{~cm}\right)$. Für zwei Werte des Fried-Parameters $r_{0, \text { oben }}$ sind jeweils Kurven für drei Windstärken (für $1 \mathrm{~ms}$ zeitlicher Verzögerung) über zunehmender Korrekturstärke aufgetragen. 
und Korrektureinstellung am DM nur $1 \mathrm{~ms}$ beträgt. Für das System KAOS ist dies etwas 'hoch gegriffen' und gilt allenfalls mit den jüngsten Aufrüstungen bei der Frequenz des Regelkreises. Die Umrechnung bereitet aber keine Schwierigkeit, da die Geschwindigkeit jeweils nur durch eine gegebene andere Verzögerungszeit in ms geteilt werden muss.

Der Versatz führt zu einer verringerten Korrekturleistung, da die Flächen konstanter Phase von Wellenfront und DM nicht mehr 'aufeinander passen' und die Subtraktion einen neuen Fehler einführt. Die Wirkung auf die Bildqualität soll nun beschrieben werden. Wir betrachten dazu die Situation im Rahmen der Taylor-Hypothese, d. h. wir wollen zunächst keine intrinsische zeitliche Entwicklung der Turbulenz und damit der Phasenbeziehungen im Phasenschirm annehmen. Die Situation ähnelt der in Abb. 3.4 für die Winkelabhängigkeit gezeigten insofern, dass dort ebenfalls ein verschobener Ausschnitt des oberen Phasenschirms zum gleichen Zeitpunkt der Korrektur zugeführt wurde. Bei der Berücksichtigung des Windes kommt die Dekorrelation der unteren Turbulenzschicht hinzu, sowie in der oberen Schicht die Überlagerung der Effekte Wind und Winkelabhängigkeit nach Stärke und Richtung.

Wenden wir uns also zunächst Abbildung 7.9 zu, wo Effekte eines laminaren Windes unmittelbar über dem Teleskop zusammengefasst sind. Wie in den vorigen Abschnitten unterscheiden wir die Situation einer Langzeitbelichtung von der mittleren Kurzzeitaufnahme eines Speckle-Bildes, wobei die Strehl-Zahlen im letzteren Fall jeweils aus der STF stammen, nicht aus den PSFs, wie sonst üblich. In den Zeichenfeldern sind jeweils drei Gruppen von Kurven aufgetragen. Innerhalb der Gruppen, die jeweils zu einem festem $r_{0, \text { unten }}$ gehören, variiert der Versatz durch Wind zwischen 0 bis $5 \mathrm{~cm}(0 \mathrm{~m} / \mathrm{s} \mathrm{bis}$ $50 \mathrm{~m} / \mathrm{s}$ ). Je nach Strukturgröße in der Wellenfront hat der Versatz größere Auswirkungen auf kleinskaligere Deformationen des DM. Deshalb sind die 'Dämpfungsfaktoren' für die Strehl-Zahlen bei Korrekturen höchster Ordnung auch am größten. Dagegen ist beispielsweise für die TT-Korrektur praktisch kein Einfluss des Windes zu bemerken, da die Korrelationslänge für die TT-Moden deutlich größer ist als die die betrachteten Versatzlängen (vgl. Abb. 3.5). Erst bei den beiden besten Korrekturniveaus meint man eine gewisse Sättigung zu bemerken. Hier ist offenbar gerade die entsprechende räumliche Skala erreicht, die sich noch zu korrigieren lohnt, d. h. das System arbeit bei dieser Kombination von WFS-Sampling, Anzahl der Spiegelmoden und Durchmesser des Teleskops (hier: $70 \mathrm{~cm}$ ) optimal effizient. Zum Fall der Kurzzeitbelichtung ist zu sagen, dass Wind hier einen generell kleineren Einfluss auf die Strehl-Verhältnisse hat, sich alle am LangzeitFall diskutierten Effekte auf einem höheren Niveau in gleicher Weise widerspiegeln und die erreichten Strehl-Zahlen bei voller AO-Korrektur nur unwesentlich höher liegen, als bei den Langzeit-OTFs.

In Abb. 7.10 ist nach bekannter Vorgehensweise die Rolle der beiden partiellen FriedParameter vertauscht. So wird nun der Fried-Parameter $r_{0, \text { unten }}$ der unteren Schicht festgehalten und jede Gruppe von Graphen in den Feldern gilt für einen Wert für die Turbulenzstärke der oberen Turbulenz. Wiederum übt die obere Turbulenzschicht nur dann deutlichen Einfluss auf die gesamte Kompensationsleistung aus, wenn die beiden FriedParameter nicht zu weit auseinander liegen. Ist dies jedoch der Fall, hat der oft sehr starke Wind unterhalb der Tropopause einen ungemein deutlichen Effekt. In Verbindung mit der Winkelabhängigkeit, die in der oberen Atmosphäre prinzipiell auf dem gleichen Dekorrelationseffekt beruht, bewirkt eine ungünstige Kombination eine merkwürdige Beobachtung: Die Speckle-Kamera mag gewöhnliche Bilder aufnehmen und das durch eine Vi- 

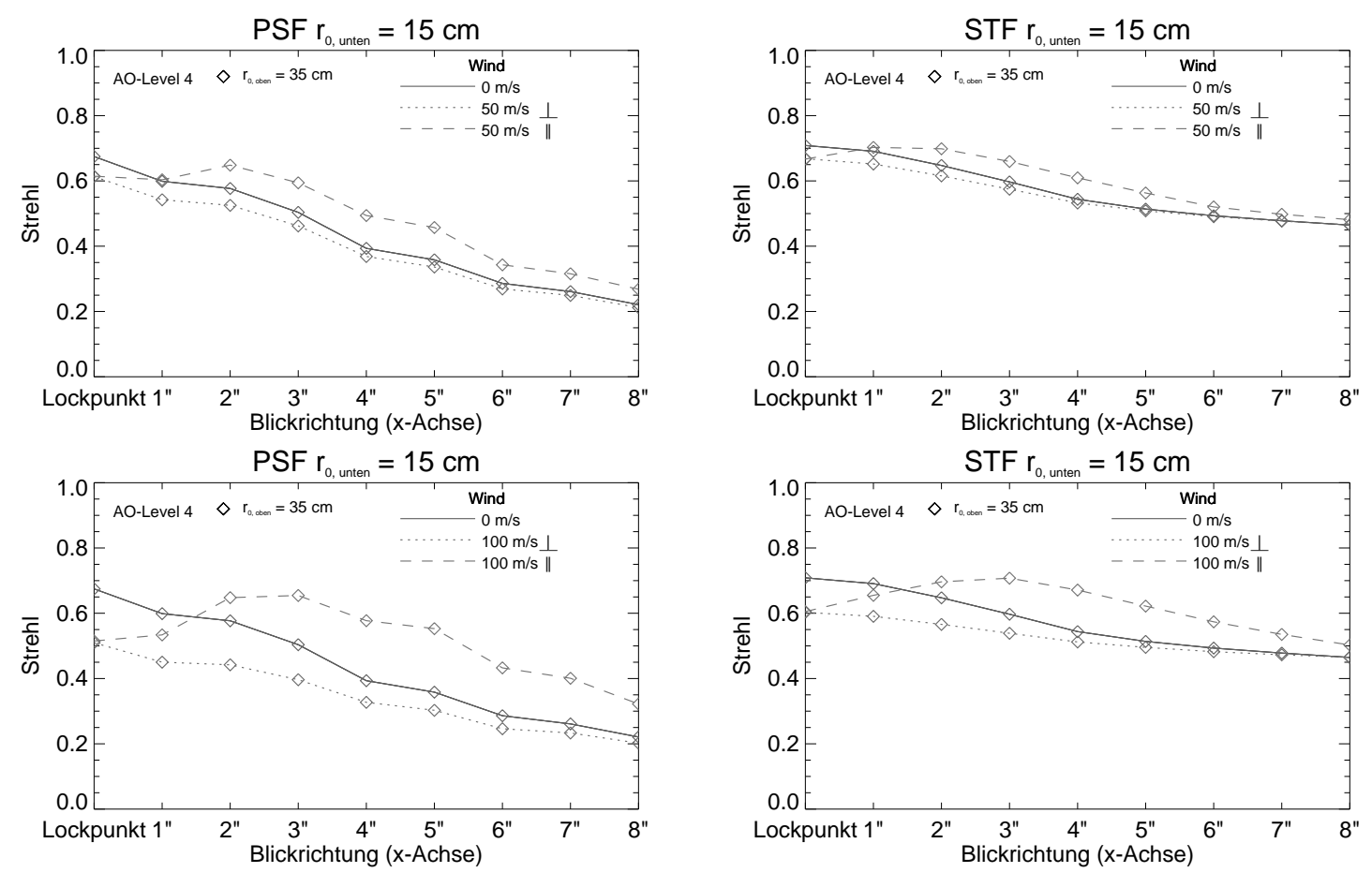

Abbildung 7.11: Zum Einfluss der Windrichtung (obere Turbulenzschicht) auf die Abbildungsqualität: Über dem Winkelabstand zum Lockpunkt (hier nur zwischen $0^{\prime \prime}$ und $8^{\prime \prime}$ ) sind die Strehl-Zahlen für die oben links spezifizierten beiden Windrichtungen bei $0^{\circ}$ und $90^{\circ}$ aufgetragen. Die linke Graphik gilt für schwachen Wind, rechts daneben die Situation für eine größere Windstärke.

deokamera betrachtete 'Live-Bild', wie es zur Kontrolle von optischen Einstellungen oft verwendet wird, zeige die üblichen Schwankungen der Schärfe und das 'Wabern' der atmosphärischen Turbulenz - Bedingungen wie sie normalerweise ideal für den Betrieb der Adaptiven Optik erscheinen. Trotzdem versagen alle Versuche, das AO-System zu einem stabilen Korrekturbetrieb zu bringen! Hier trägt der Wind die Turbulenzzellen so schnell am Teleskop vorüber, dass den Spiegel nach erreichter Endposition der Korrekturstellung jeweils fast vollständig dekorrelierte Phasenschirme erreichen. Somit ist keinerlei Verbesserung der Abbildung zu bemerken und die Korrelationsfunktionen zur WF-Messung werden so verrauscht, dass keine deutlichen WFS-Signale mehr daraus gewonnen werden können. Dies bewirkt eine Reduktion des Korrekturlevels und im ungünstigsten Fall zum 'Aussteigen' bzw. Abschalten des Kontrollkreises.

Schließlich kommen wir zur Abhängigkeit von der Windrichtung in der oberen Turbulenzschicht. Dazu sind in Abb. 7.11 bei festen Fried-Parametern für beide Schichten wieder links die Situation bei der Langzeit-PSF dargestellt, recht für die kurzen Belichtungszeiten. In den unteren Feldern ist jeweils der doppelte Phasenschirm-Versatz der oberen Graphiken berücksichtigt, also bei der angenommenen Verzögerung von nur $1 \mathrm{~ms}$ Geschwindigkeiten von $50 \mathrm{~m} / \mathrm{s}$ bzw. $100 \mathrm{~m} / \mathrm{s}$. Hier beachte man außerdem die feinere Skala in der Abszisse.

Die Kurven für die Windrichtung rechtwinklig zur betrachteten Variation des Blickwin- 
kels zeigen natürlich die oben besprochenen Dekorrelationserscheinungen und die Graphen liegen für alle Winkel niedriger, als die zu Windstärke Null gehörigen. Dagegen verschiebt sich, wenn die Winkelabhängigkeit in Richtung des Windes betrachtet wird, das Maximum der Kurve von der Richtung des Lockpunktes weg, und zwar um einen Winkel proportional zur Windgeschwindigkeit. Nach den bisherigen Ausführungen wird klar, welchen Effekt wir hier sehr schön sehen. Dadurch, dass wir den Wind nach der Taylor'schen Hypothese betrachten, verschiebt sich der vom WFS gemessene Ausschnitt des Phasenschirms an eine Position, die bei gegebener Höhe der Turbulenzschicht eben dem Winkel entspricht, bei dem in der Abbildung 7.11 das Maximum gemessen wird! Hat man umgekehrt eine empfindliche Methode, um Strehl-Zahlen aus Beobachtungen zu gewinnen, bietet sich hier auch die Möglichkeit der Windmessung!

Damit beschließen wir die Betrachtungen der Parameterabhängigkeiten der mit dem Simulations-Code berechenbaren Größen und wenden uns im nächsten Abschnitt einem Anwendungsbeispiel zu, wie die STFs aus der Simulation auf Beobachtungsdaten angewendet werden können.

\subsection{Rekonstruktion von AO-Speckle-Daten}

Der herkömmliche Göttinger Speckle-Rekonstruktionscode schätzt isoplanare Ausschnitte des unaberrierten Bildes eines Beobachtungsobjektes in der Annahme richtungsunabhängiger Statistik für die WF-Aberrationen aus einer Serie von etwa 100 Kurzzeitbelichtungen ab. Ausgangspunkt ist dabei die isoplanare, inkohärente Abbildungsgleichung (2.21), die im Fourierraum als Produkt geschrieben werden kann:

$$
F\left(s, t_{i}\right)=F_{0}(s) \cdot O T F\left(s, t_{i}\right) .
$$

Der Vektor $s$ bedeutet darin die räumliche Frequenzkoordinate und $t_{i}$ den Aufnahmezeitpunkt des $i$-ten Bildes des Speckle-Bursts mit Fouriertransformierter F. $F_{0}$ bezeichnet dagegen das Spektrum des Beobachtungsobjektes. Typisch für die Speckle-Rekonstruktion ist nun, dass die Fourierphasen und -amplituden getrennt voneinander bestimmt werden (von der Lühe 1993). Bei der Phasenbestimmung findet das sogenannte 'Speckle masking' (Weigelt 1977, Lohmann et al. 1983) Anwendung, wobei insbesondere auf die Behandlung von Rauschen geachtet wurde (de Boer 1996). Für die Wiederherstellung der Amplituden wird die Methode nach Labeyrie (1970) verwendet, wobei mit dem 'Spektralen Quotienten' (von der Lühe 1984) und Modell-Übertragungsfunktionen nach Korff (1973) gearbeitet wird, da auf der Sonnenoberfläche keine a priori bekannten, konstanten Referenzstrukturen vorhanden sind. Man nutzt hier die Tatsache aus, dass das mittlere Leistungsspektrum der momentanen OTF, also die Speckle-Transferfunktion

$$
\operatorname{STF}(\boldsymbol{s})=\left\langle\left|O T F\left(\boldsymbol{s}, t_{i}\right)\right|^{2}\right\rangle
$$

im Gegensatz zum Langzeit-Mittelwert im gesamten Frequenzbereich bis über die Beugungsgrenze hinaus nicht verschwindet. Mit Gleichung 7.1 erhält man dann, das Rauschen ausgeklammert, das Leistungsspektrum des 'wahren Bildes', und damit seine Fourieramplituden, durch

$$
\left|F_{0}(s)\right|^{2}=\frac{\left\langle\left|F\left(s, t_{i}\right)\right|^{2}\right\rangle}{S T F(s)} .
$$



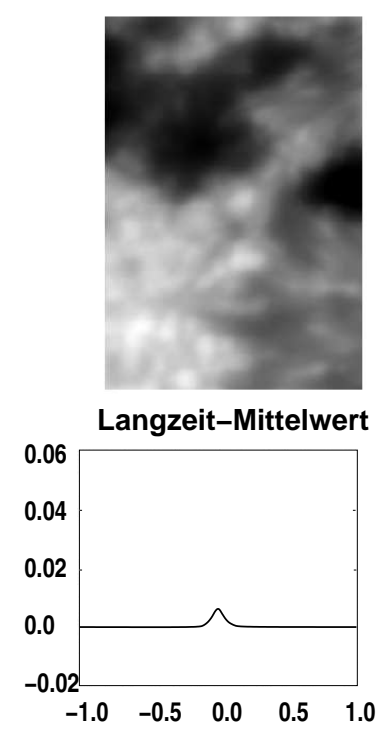
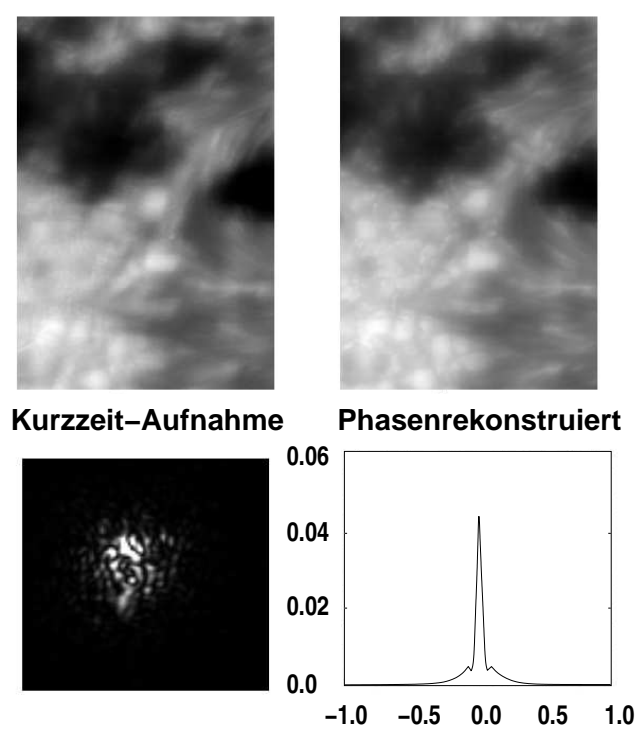
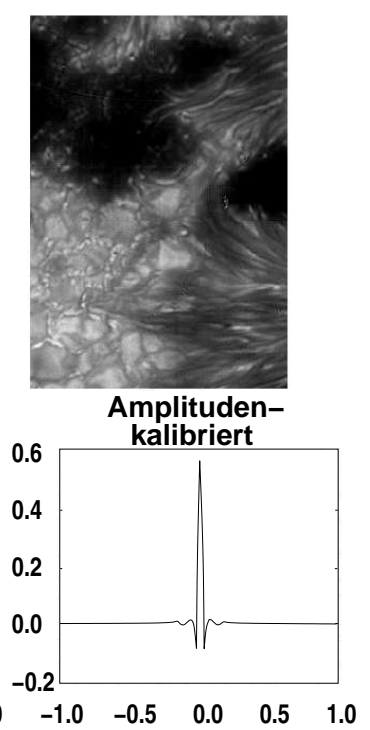

Abbildung 7.12: Stationen im Speckle-Bildrekonstruktionsprozess. Dargestellt für ein Beispielbild in der oberen Reihe mit zugehörigen, repräsentativen PSFs in der unteren Reihe. Von links nach rechts ist: Ganz links die aus 100 Einzelbildern gemittelte 'Langzeitbelichtung' (Belichtungszeit etwa 1 s), daneben ein Einzelbild der SpeckleAufnahmeserie (10 ms Belichtungszeit), dann das phasenrekonstruierte Bild, das bereits alle Feinstrukturen in endgültiger Auflösung und an der richtigen Position hat, und schließlich ganz rechts die amplitudenverstärkte, fertige Rekonstruktion. Die PSF für das Einzelbild illustriert das Grundphänomen der Methode: das Speckle-Muster einer Punktlichtquelle, das trotz großer Streuung der Lichtenergie noch Interferenzstrukturen von Ausdehnung eines Airy-Scheibchens zeigt. Auf die Negativitäten der repräsentativen PSF der fertigen Rekonstruktion wird im Text näher eingegangen.

Die Division durch die STF kompensiert die Effekte der Atmosphäre und der theoretischen, beugungsbegrenzten Modulations-Übertragungsfunktion $(M T F=|O T F|)$ des Teleskops! Im Idealfall ist das resultierende Bild dasjenige, das ein hypothetisches Instrument erzeugen würde, dessen MTF bis zur Beugungsgrenze konstant wäre und außerhalb auf Null abfiele ${ }^{1}$. Solch ein Instrument ist physikalisch nicht realisierbar. Die effektive PSF ist dann keine Airy-Figur, sondern wird durch eine Funktion beschrieben, deren Betragsquadrat die Airy-Figur ist, und sie hat zudem negative Seitenflügel! Die Anwendung der hier erläuterten Vorgehensweise zur Wiederherstellung der Bildinformation führte bei kompakten, punktförmigen Lichtquellen in der Nachtastronomie zu 'negativer Intensität'. Die Quelle dieser Negativität ist hier das Gibb'sche Phänomen, das auftritt, wenn ein Signal rekonstruiert wird, dessen spektraler Informationsgehalt über die Bandbreite des Empfängers hinausreicht. Deshalb ist dieses Verhalten nicht beliebt, ist doch die nicht-Negativität von Bildern eine sehr mächtige Quelle für nichtlineare Bildrekonstruktionsalgorithmen! Man umgeht dieses Problem jedoch ganz einfach durch Multiplikation der Fouriertransformierten mit der beugungsbegrenzten MTF. Dies eliminiert die Negativität in der Re-

\footnotetext{
${ }^{1}$ anders gesagt die MTF einer unendlich großen Teleskopöffnung mit optischem Low-pass für Frequenzen unterhalb der Beugungsgrenze
} 
konstruktion.

Im Fall der Sonnenbeobachtung allerdings sieht die praktische Konsequenz weniger ernst aus. Die Intensität in den rekonstruierten Bildern solarer Szenen ist immer positiv, da sich die kleinskaligen Strukturen vor einem Hintergrund hoher Lichtintensität befinden. Deshalb ist die Negativität (hier) kein Hinderungsgrund für die Methode. Vielmehr erzeugt die Speckle-Rekonstruktion auf die beschriebene Weise ein aussagekräftiges Resultat, wenn die beobachtete Struktur frequenzlimitiert ist und damit für das beugungsbegrenzte Teleskop auflösbar ist. Die Intensitätsverteilung bleibt nämlich in diesem Fall auch dann gleich, wenn mit einem sehr viel größeren, beugungsbegrenzten Teleskop beobachtet wird. Dies ist etwa der Fall, wenn Granulation in 'ruhigen' Gebieten der Photosphäre der Sonne mit einem Teleskopdurchmesser von etwa einem Meter beobachtet wird.

Hat man es dagegen mit unaufgelösten Strukturen und Einzelheiten zu tun, deren Raumfrequenzen über den wiederhergestellten Frequenzbereich hinausreichen, nimmt man zwar Unsicherheiten in der Intensitätsmessung in Kauf, jedoch bleiben diese auch dann gleich groß, wenn wir die beugungsbegrenzte MTF in die Rechnung miteinbeziehen! In dieser Weise ist das so rekonstruierte Bild in dem Sinn photometrisch korrekt, dass seine Intensitätsvarianz diejenige des Objektes korrekt wiedergibt (integriert über das Frequenzband der Restaurationsmethode). In der Praxis ist die effektive MTF durch die RauschfilterFunktion gegeben, deren inverse Fouriertransformierte die effektive PSF liefert.

Man benötigt noch eine Abschätzung der STF, die repräsentativ für das Spektrum der unbekannten momentanen OTFs während der Aufnahmezeit stehen. Mit dem Korff'schen Modell für den Einfluss atmosphärischer Aberrationen kann zwar für alle Turbulenzzustände eine STF berechnet werden, wobei angenommen wird, dass die Atmosphäre durch den Fried-Parameter vollständig charakterisiert werde, aber zur Auswahl der 'richtigen' STF ist noch ein Vergleich zwischen Modell und Experiment nötig. Dies leistet der spektrale Quotient $\epsilon$, der nach

$$
\epsilon(s)=\frac{\left|\left\langle F\left(s, t_{i}\right)\right\rangle\right|^{2}}{\left\langle\left|F\left(s, t_{i}\right)\right|^{2}\right\rangle}=\frac{\left|\left\langle O T F\left(s, t_{i}\right)\right\rangle\right|^{2}}{\left\langle\left|O T F\left(s, t_{i}\right)\right|^{2}\right\rangle}
$$

aus den beobachteten Größen $F$ sowie Modell-OTFs berechnet werden kann. Er ermöglicht die Bestimmung des Fried-Parameters und damit der am besten auf die reale Situation passenden OTF.

Aus dem Gebrauch einer Adaptiven Optik ergeben sich allerdings Veränderungen in der Strukturfunktion, die die durchschnittliche Phasendifferenz zwischen zwei Punkten der Wellenfront angibt. Die Phasenunterschiede zur idealen WF sollen ja nach Möglichkeit auf Null gedrückt werden. Im Korff'schen Modell taucht die Strukturfunktion aber bei der Berechnung der STF auf, d.h. als Konsequenz müssen neue Modell-STFs berechnet werden, in denen sich die Dynamik der AO-Korrektur widerspiegelt. Abweichungen im spektralen Verlauf der STF führen ansonsten nach Gleichung 7.3 zu Fehlern in den rekonstruierten Intensitäten des beobachteten Objekts. Ziel meiner Simulationen ist nun insbesondere, die wichtigsten Parameter des verwendeten AO-Systems bei der Berechnung von Speckle-Transferfunktionen mit einzubeziehen, um eine bessere Zuverlässigkeit bei der Rekonstruktion von Helligkeits- und Kontrastwerten zu erreichen. Immerhin schlagen sich AO-induzierte Veränderungen nur in den Modell-STFs nieder, da das Speckle-Masking-Bispektrum, und damit die Phasen-Rekonstruktion, durch die veränder- 
te WF-Fehlerstatistik nicht beeinflusst wird. Die Amplituden werden jedoch abhängig von der Anzahl korrigierter Moden um einen frequenzabhängigen Faktor angehoben (siehe etwa Wang \& Markey 1978). Hinzu kommt, dass die Kompensation des Wellenfrontfehlers durch eine $\mathrm{AO}$ noch weit davon entfernt ist, perfekt zu sein, und somit einen weiteren Faktor darstellt, der die OTF über die Frequenz ungleichmäßig verändert. Prinzipiell hat man es also mit Übertragungsfunktionen ungewöhnlicher Struktur zu tun, verglichen mit denen rein atmosphärischen Einflusses, wie es im Korff"schen Modell angenommen wird. In der von K. Puschmann und mir durchgeführten Studie (Puschmann \& Sailer 2006) wurden deshalb zwei Modifikationen im ursprünglichen Rekonstruktionscode an Hand der in Abschnitt 6.3 beschriebenen Beobachtungsdaten vom schwedischen Sonnenteleskop untersucht und die Ergebnisse zusammen mit den Resultaten der konventionellen Version verglichen. Dies soll im Folgenden wiedergegeben werden.

\subsubsection{Modifikation des Programmpakets}

Der auffälligste Effekt beim Einsatz der AO ist die Feldabhängigkeit der Kompensationsleistung. Dies macht sich in der bildfeldabhängigen, durchschnittlichen Schärfe der Beobachtungsdaten bemerkbar, wobei die beste Bildqualität üblicherweise im isoplanaren Gebiet um den Lockpunkt der AO herum erreicht wird. Nur für diese Blickrichtung liegen ja bei einer konventionellen Adaptiven Optik Informationen des Wellenfrontsensors vor (vgl. Abschnitt 3.4.5). In das Korff'sche Modell übertragen, wird dadurch die Annahme der Isotropie in der Statistik der Wellenfrontfehler verletzt, d. h. die Strukturfunktion wird feldabhängig und neue, 'nicht-atmosphärische' STFs müssen berechnet werden. In der Implementierung der Speckle-Rekonstruktion muss diese Feldabhängigkeit sozusagen digitalisiert werden, da jeweils nur kleine Ausschnitte des Bildfeldes den Rekonstruktionsprozess durchlaufen dürfen: Das gesamte Bildfeld erscheint ja normalerweise unter einem bei weitem größeren als dem isoplanatischen Winkel. Deshalb werden die Bilder in isoplanare Ausschnitte unterteilt, die separat rekonstruiert werden und danach wieder zum vollständigen Bild zusammengesetzt werden. Dabei ist ein ausreichender Überlapp zu berücksichtigen, damit ein gewisser Spielraum zur optimalen Anpassung der Teilbilder untereinander gewährleistet ist. Für den hier verwendeten Datensatz haben wir eine Unterteilung in $17 \times 11=187$ Ausschnitte der Größe $5^{\prime \prime} .12 \times 5^{\prime \prime} .12(128 \times 128$ Pixel $)$ gewählt. Auf diese Weise besteht grundsätzlich die Möglichkeit, für die Rekonstruktion jedes Teilbilds eine individuelle STF zu verwenden, die von der relativen Lage zum Lockpunkt abhängt.

\subsubsection{Bestimmung des Lockpunktes}

Als Referenzpunkt zur Berücksichtigung der Winkelabhängigkeit der AO-Korrektur muss im Bildfeld die Position des Lockpunktes der AO bekannt sein. Ist dabei nicht bekannt, mittels welcher Struktur der WFS arbeitet, muss ein zuverlässiges Maß zur Ermittlung dieser Position aus den Daten selbst gefunden werden. Hier bietet sich die Varianz der differentiellen Bildbewegung an. Man misst dabei über die $N=100$ Zeitschritte der einzelnen isoplanaren Teilbildserien die residuelle Bildbewegung, nachdem zuvor die globalen Verschiebungen des ganzen Bildfeldes abgezogen wurden. Der Lockpunkt sollte dem Ausschnitt zugeordnet werden, der die kleinste Varianz der Bildbewegung aufweist. 

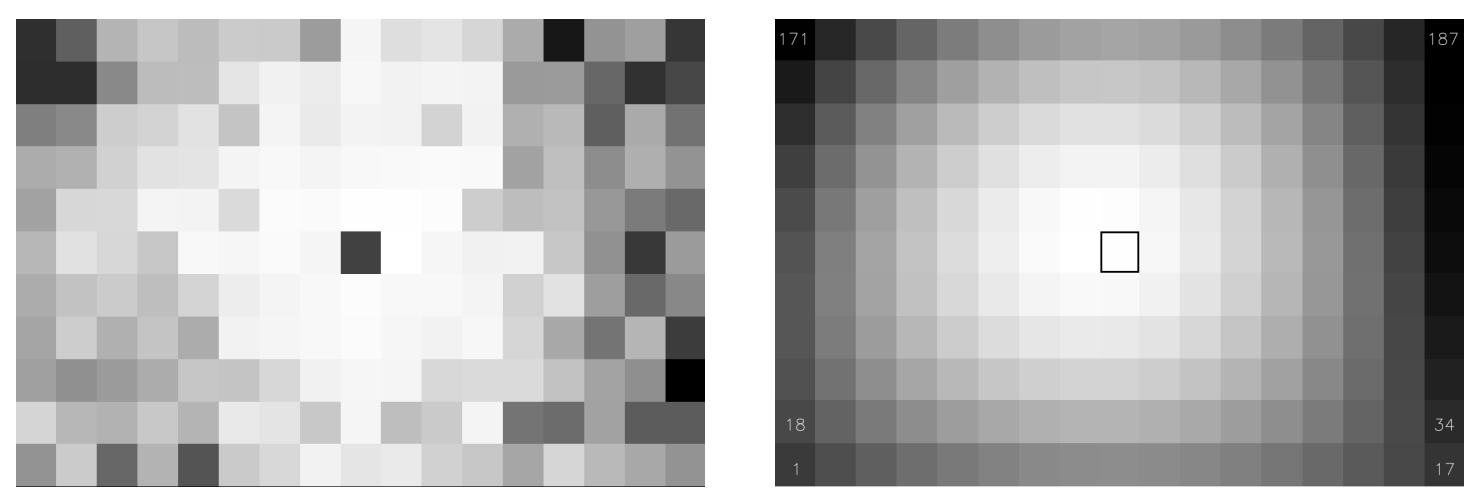

Abbildung 7.13: Zur Bestimmung des Lockpunktes der AO aus den Beobachtungsdaten. Links: Varianz des Bildversatzes der 187 isoplanaren Ausschnitte des Gesichtsfeldes aus 100 Zeitschritten. Rechts: Verteilung aus einem Fit zweiter Ordnung an die gemessene Verteilung links. Dunklere Grautöne bedeuten höhere Varianz. Das eingerahmte Subfeld enthält demnach den Lockpunkt.

Allerdings stellt sich als zuverlässigere Methode heraus, die zufallsbehaftete Verteilung der Varianzwerte über das Bildfeld vorher zu glätten oder die Minimumsposition eines Fit zweiter Ordnung zu verwenden. Das Ergebnis ist in Abbildung 7.13 dargestellt.

Zwei Punkte müssen hier noch beachtet werden: Zum Einen besitzen die beiden Spiegel TT und DM am SST jeweils ihren eigenen Lockpunkt. Beide sollten schon bei der Beobachtung übereinstimmend gewählt werden, da sonst die Möglichkeit bestünde, dass in der oben beschriebenen Vorgehensweise ein "falscher" globaler Bildversatz abgezogen wird. Dies hat für die Korrektur der Bildbewegung an sich keine Konsequenzen, führt jedoch in der Folge zu Fehlern und Schwierigkeiten bei der Modellierung der Winkelabhängigkeit der Aberrationen höherer Ordnung, da nicht die dafür relevante Referenzrichtung des deformierbaren Spiegels bestimmt wird, sondern die des TT-Spiegels.

Der zweite Punkt hat Bedeutung für die Simulation, wo die atmosphärischen Bedingungen zum Aufnahmezeitpunkt modelliert werden. Im Falle eines starken Windes in der oberen Turbulenzschicht wird der vom WFS erfasste Ausschnitt der Turbulenz fortgetragen und liegt zum Zeitpunkt der tatsächlichen Korrektur durch den DM auf einer anderen Sichtlinie, die einem anderen Bildausschnitt entspricht. Das ganze Muster der winkelabhängigen Korrektur ist somit in den Beobachtungen verschoben. Falls also während der Beobachtung notiert wird, welches Target der WFS benutzte, könnte umgekehrt aus den aufgenommenen Daten im Nachhinein ein Rückschluss auf die mittleren Windverhältnisse der oberen Atmosphäre geschlossen werden.

\subsubsection{Modellierung der Richtungsanisotropie}

In unserer Arbeit nahmen wir als erste Näherung nur eine radiale Bildfeldabhängigkeit der Speckle-Transferfunktion an. Hat man den Lockpunkt gefunden, kann das Bildfeld nach Winkelabstand zu dieser Referenzrichtung aufgeteilt und die entsprechenden Teilbildserien in Gruppen eingeteilt werden. Somit wird für alle Ausschnitte, deren Winkelabstand zum Lockpunkt der AO innerhalb eines gewissen Intervalls liegt, diesselbe STF benutzt. Dieser Schritt soll mit Abbildung 7.14 illustriert werden. Zur Aufteilung gingen wir dabei 


\section{Gesamtes Gesichtsfeld der SST-Daten}

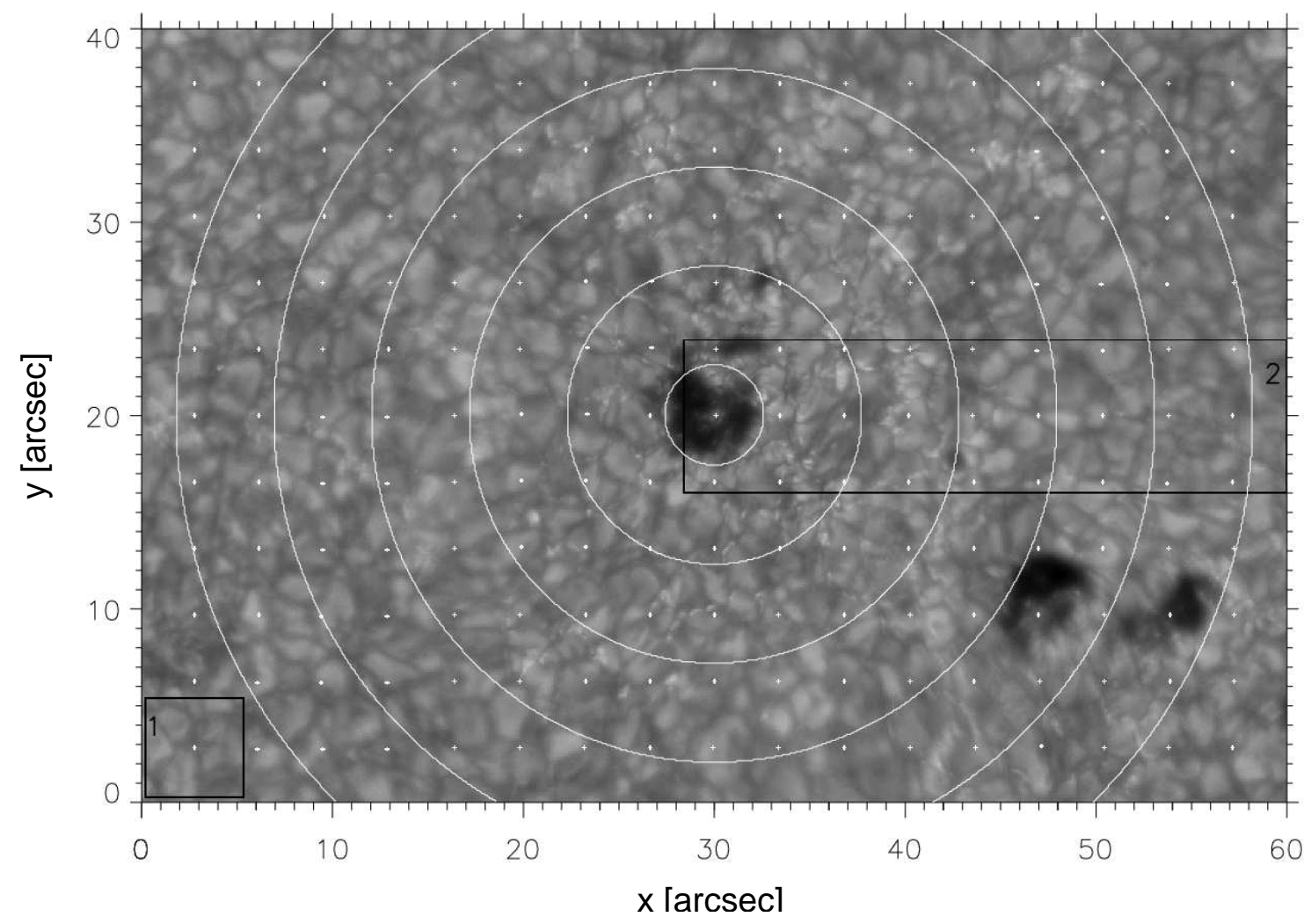

Abbildung 7.14: G-Band Rohbild des am Swedish 1-m Solar Telescope beobachteten aktiven Gebiets NOAA AR0636. Zur Berücksichtigung der blickrichtungsabhängigen AO-Korrektur wurden die zu rekonstruierenden Teilbildserien je nach Winkelabstand ihres Mittelpunktes (Punktgitter in weiß) zum Lockpunkt der AO in Gruppen eingeteilt, die auf Kreisringen von der 'Breite' 5"'12 (zwischen benachbarten Kreisen) liegen. Der Ausschnitt 1 (links, unten) zeigt die Größe eines Teilbildes und anhand von Ausschnitt 2 (rechts, mitte) wird im Folgenden die Analyse illustriert werden.

so vor, dass zentriert über dem Lockpunkt der AO ein Kreis vom Durchmesser 5"'12 den Bereich der bestmöglichen WF-Korrektur abgrenzt. Der gewählte Wert für den Durchmesser stellt eine Schätzung des isoplanaren Winkels dar und beruht auf Erfahrung. Das zentrale Gebiet wird im Folgenden als 'Ring 1' bezeichnet und umfasst das Bild einer solaren Pore, die im Übrigen auch die tatsächliche Referenzstruktur des WFS während der Beobachtung bildete. Sechs konzentrische Kreisringe ('Ring 2 bis 7') der Breite 5".12 umgeben die Zentralregion und werden als Gebiete angesehen, in denen sich der spektrale Quotient, und damit die STF, nicht ändert. Die Einteilung in diese sieben Gruppen erfolgt nun auf Grund der Lage der Zentrumspositionen ihrer Teilbildserien, die in Abb. 7.14 als weiße Punkte dem Bild überlagert sind: liegt das Zentrum in einem gewissen Ring, wird auch das Teilbild diesem Ring zugeordnet. 


\subsubsection{Drei Näherungen}

Für das weitere Vorgehen erinnern wir uns an das eigentliche Ziel, nämlich solche STFs zur Rekonstruktion zu verwenden, die möglichst gut die Bedingungen während der Beobachtung widerspiegeln sollten, also die durch die AO veränderte Statistik von Wellenfrontstörungen in einer bildfeldabhängigen Weise modellieren sollten. In Annäherung an dieses Ziel wurden die folgenden drei Ansätze zur Rekonstruktion verfolgt und miteinander verglichen:

- Ansatz A: Konventionelle Rekonstruktion

Annahme rein atmosphärisch bedingter Aberrationen ermöglicht die Berechnung der STFs nach Korff. Die weitere Annahme einer isotropen Strukturfunktion führt zu konstanter Turbulenzstärke über das gesamte Bildfeld, also einem einzigen Wert für den Fried-Parameter. Letzterer kann also durch Mittelung der spektralen Quotienten aller Teilbildserien bestimmt werden.

- Ansatz B: 'Anisotrope Atmosphäre'

Die Annahme rein atmosphärischer Störungen der Wellenfront bleibt wie in Methode A, d.h. konventionelle STFs können verwendet werden. Dabei wird angenommen, dass sich die AO-Korrektur einfach als allgemeine Verbesserung der SeeingBedingungen bemerkbar macht. Erste Näherung für eine anisotrope Strukturfunktion: Radiale Abhängigkeit von $r_{0}$ und Berechnung entsprechend der Geometrie von Abbildung 7.14 durch Mittelung über die spektralen Quotienten der Teilbildserien jeder Gruppe.

- Ansatz C: Rekonstruktion mit STFs aus der AO-Simulation.

STF-Berechnung mit Hilfe von Simulationen atmosphärisch induzierter Phasenverzögerungen unter Berücksichtigung der Blickrichtung und WF-Kompensation durch Adaptive Optik; damit volle Berücksichtigung der Veränderungen in der effektiven Turbulenzstatistik incl. Feldabhängigkeit. Die Annahmen und Vereinfachungen stecken nun jedoch in der Modellbildung der Simulation und in der Anpassung an experimentelle Beobachtungsdaten.

\subsubsection{Ansatz A und B - Korff'sche STFs}

In den Rekonstruktionen nach den Ansätzen A und B werden konventionelle, rein atmosphärisch beeinflusste STFs verwendet, die nach der Methode des spektralen Quotienten ermittelt werden. In Abbildung 7.15 sind nach Ansatz B die azimutal gemittelten spektralen Quotienten $\epsilon$ der sieben Kreisringe aus Abb. 7.14 über der relativen Wellenzahl aufgetragen. Bis auf Ring 1 vermindert die Mittelung von $\epsilon$ die Rauschgrenze ausreichend, sodass für jeden Kreisring ein zuverlässiger Schätzwert für den Fried-Parameter abgeleitet werden kann. $r_{0}$ folgt aus dem Vergleich des gemessenen Spektralen Quotienten mit solchen, die aus Modellen für die Übertragungsfunktionen stammen. Dabei werden diejenigen Frequenzwerte $k_{\epsilon}$ ermittelt, bei denen der Quotient $\epsilon$ aus den Daten jeweils auf die Werte 0.5, 0.3, 0.2 und 0.1 abgefallen ist. Die $k_{\epsilon}$ bilden Schätzwerte für die Seeing-Abschneidefrequenz, die mit dem Fried-Parameter über ein Potenzgesetz

$$
r_{0} / D=a \cdot\left(\frac{k_{\epsilon}}{D / \lambda}\right)^{b}
$$




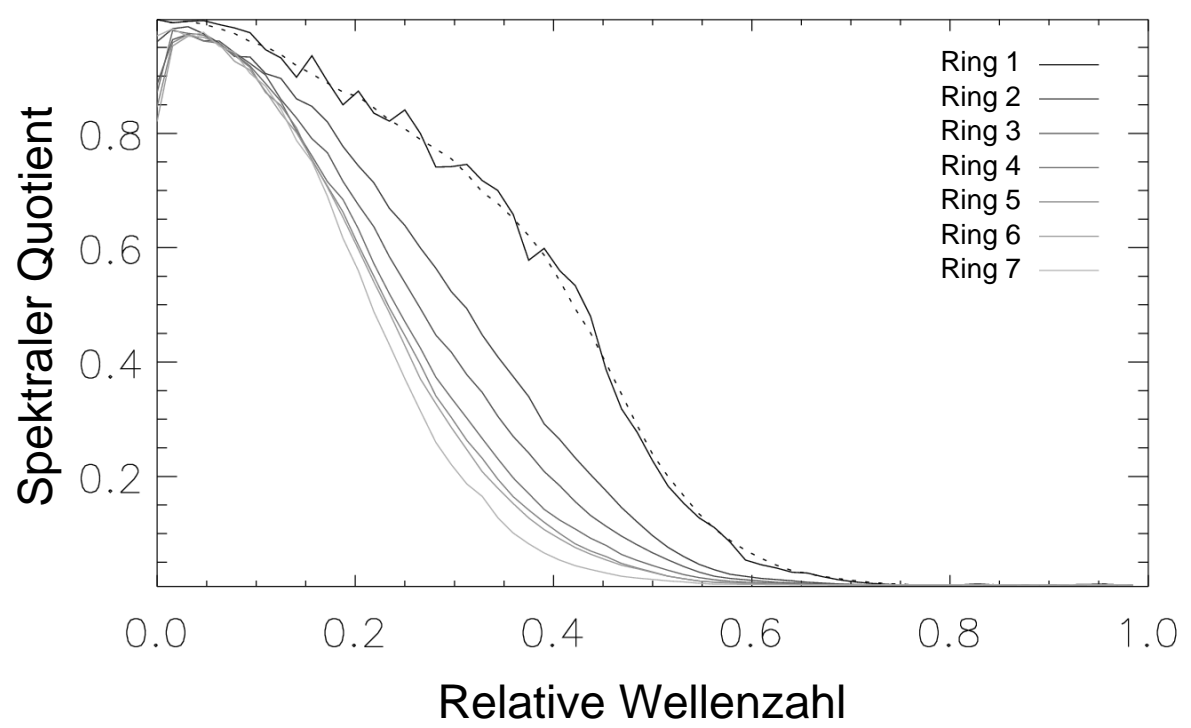

Abbildung 7.15: Azimutal gemittelte Spektrale Quotienten der sieben Kreisringe aus Abbildung 7.14 nach Ansatz B, aufgetragen über der relativen Wellenzahl (normiert auf die Beugungsgrenze $D / \lambda$ ). Der Quotient von Ring 1 (Bereich des Lockpunktes) resultiert aus einer einzigen Teilbildserie und wurde mittels eines Boxcar-Fensters von 7 Pixel im Frequenzraum geglättet, um den Einfluss von Rauschen zu minimieren.

zusammenhängt, dessen Variablen a und b schwach von $\epsilon$ abhängen (de Boer 1993) und die tabelliert vorliegen (siehe Tabelle 7.1). Aus den vier Schätzwerten für den Fried-

\begin{tabular}{|l|c|c|c|c|}
\hline Spektraler Quotient $\epsilon$ & 0.5 & 0.3 & 0.2 & 0.1 \\
\hline Koeffizient a & 1.030 & 0.753 & 0.626 & 0.492 \\
\hline Exponent b & 1.063 & 0.992 & 0.939 & 0.873 \\
\hline
\end{tabular}

Tabelle 7.1: Koeffizienten der Beziehung 7.5 zwischen relativer SeeingAbschneidefrequenz (normiert auf die Beugungsgrenze $D / \lambda$ ) und normiertem Fried-Parameter $\alpha=r_{0} / D$. Entnommen aus de Boer (1993).

Parameter kann im Programmpaket zwischen Maximal-, Minimal- und Durchschnittswert gewählt werden, der dann eindeutig die STF bestimmt, die zur Rekonstruktion verwendet werden soll (siehe Abb. 7.16). Um eine übermäßige Verstärkung der Fourieramplituden bei der Rekonstruktion zu vermeiden, benutzten wir jeweils die größten Schätzwerte für $r_{0}$. Die Anzahl der zur Mittelung zur Verfügung stehenden Teilbildserien, sowie die ermittelten Werte für die Turbulenzstärke sind in Tabelle 7.2 unter den drei Wellenlängen aufgelistet, für die eine simultane Aufnahmeserie zu weiteren Analysen vorliegt. Ein zunächst schneller Abfall des gemessenen $r_{0}$ wird bei wachsendem Abstand zum Lockpunkt in allen drei Wellenlängen beobachtet. Bei größeren Winkelabständen, etwa ab dem fünften 


\begin{tabular}{|l|ccccccc|c|}
\hline Kreisring & 1 & 2 & 3 & 4 & 5 & 6 & 7 & Alle (Ansatz A) \\
\hline Anzahl der Teilbildserien & 1 & 20 & 24 & 44 & 41 & 41 & 16 & 187 \\
\hline$r_{0}$ bei $\lambda=4305 \AA$ & 48 & 34 & 30 & 27 & 26 & 25 & 23 & 27 \\
$r_{0}$ bei $\lambda=4507 \AA$ & 52 & 39 & 35 & 32 & 30 & 30 & 30 & 32 \\
$r_{0}$ bei $\lambda=6020 \AA$ & 64 & 54 & 49 & 45 & 43 & 43 & 41 & 45 \\
\hline
\end{tabular}

Tabelle 7.2: Berechnete Fried-Parameter nach Ansatz B für die Kreisringe aus Abb. 7.14 sowie nach Ansatz A für das gesamte Bildfeld. Die Spektralen Quotienten werden jeweils aus einer Teilbildserie gewonnen und alle Quotienten eines Ringes (bzw. des gesamten Bildfeldes) gemittelt. Die Resultate sind in Abb. 7.15 aufgetragen.

Kreisring, sieht man dagegen gleichbleibende Werte in allen Wellenlängen, was mit dem isoplanaren Winkel zusammenhängt und mit einer abfallenden Kompensationsleistung bezüglich der oberen Turbulenzschicht erklärt werden kann. Die Werte für den FriedParameter bleiben dabei außerordentlich hoch, was verwundert, da solche Zahlen im unkorrigierten Fall nur sehr selten gemessen werden. In unserem Zwei-Schichten-Modell für die atmosphärische Turbulenz können wir diesen Effekt jedoch zum Teil folgendermaßen erklären: die WF-Störungen der unteren Turbulenzschicht treten nahe der Teleskopöffnung auf, wo deswegen Wellenfronten aus allen Richtungen innerhalb des Blickfeldes fast dieselben Turbulenzzellen durchqueren. Werden diese Deformationen durch eine AO korrigiert, bewirkt dies folglich im gesamten Blickfeld eine Verbesserung der Bildqualität. Hinzu kommt, dass die bodennahe Schicht die größte Turbulenzstärke aufweist und der WFS zudem in der optisch konjugierten Ebene dieser Schicht arbeitet. Dies bewirkt, dass nahe dem Teleskop verursachte WF-Störungen am genauesten und viel deutlicher gemessen werden können, als Störungen der oberen Atmosphäre, die gewissermaßen defokussiert erscheinen. Wir haben deshalb Grund zu der Annahme, dass in den äußeren Teilen des Bildfeldes tatsächlich eine signifikante Wellenfrontkorrektur beobachtet wird, die sich in höheren Werten von $r_{0}$ bemerkbar macht.

\subsubsection{Ansatz C - Simulation der AO-Korrektur}

Modellatmosphäre Bei bekannter Phase der Wellenfrontrealisierungen an der Teleskopöffnung kann die momentane OTF mittels FFT als Autokorrelationsfunktion der WF berechnet werden. Insbesondere kann nach derselben Methode mit der residuellen WF nach simulierter AO-Kompensation verfahren werden, wie in Abschnitt5.4 beschrieben wurde. Im Gegensatz zu den beiden Ansätzen A und B können deshalb in Ansatz C eine ganze Reihe von Parametern berücksichtigt werden, deren Beschreibung bei der theoretischen Berechnung praktisch nicht möglich wären.

Die Werte der wichtigsten Parameter für die Simulation der Phasenschirme zur Berechnung der STFs können folgendermaßen zusammengefasst werden:

- Ein Zwei-Schichten-Modell für atmosphärische Turbulenz wird angenommen, wobei neben der bodennahen Turbulenz (Höhe: $0 \mathrm{~m}$ über der Teleskopöffnung) die 


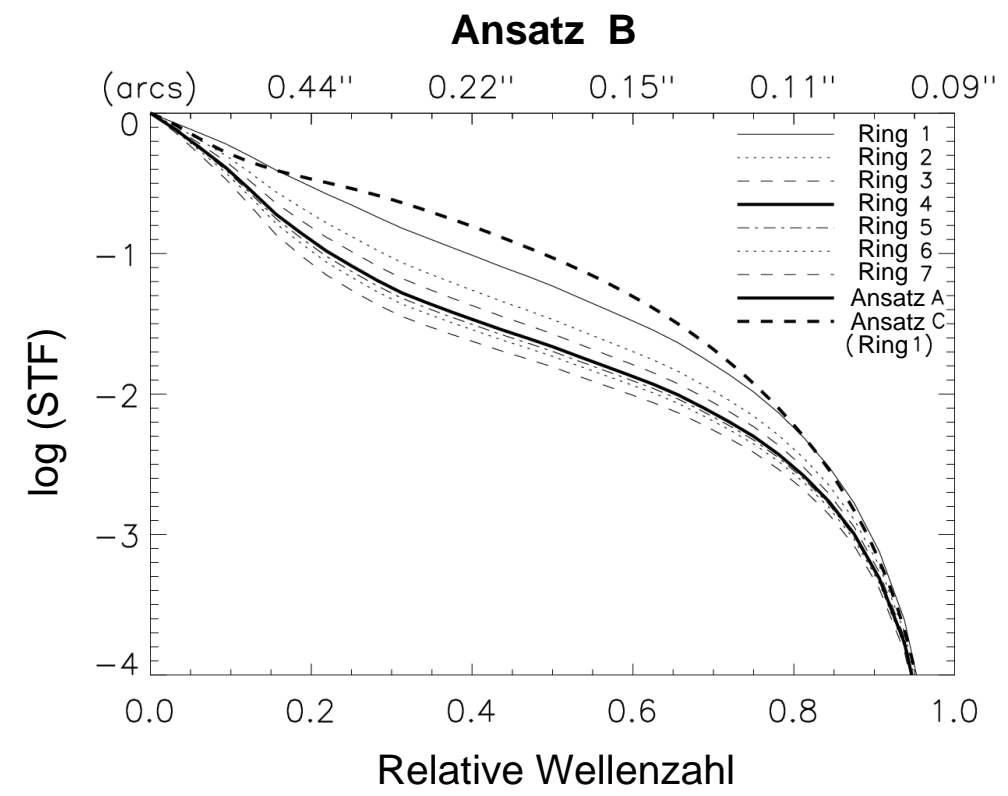

Abbildung 7.16: Logarithmische Darstellung der in Ansatz B benutzten Korff'schen Modell-STFs, aufgetragen über der auf das Beugungslimit $D / \lambda$ normierten Winkelfrequenz bzw. den entsprechenden Winkeln (obere Abszissenteilung). Der Wert des FriedParameters für Ring 4 ist fast gleich dem von Ansatz A, deshalb sind die verwendeten STFs identisch. Zum Vergleich der Signalstärke ist zusätzlich die in Ansatz C für die Lockpunktregion verwendete STF eingezeichnet.

Turbulenz der freien Atmosphäre mit einer Schicht in $8000 \mathrm{~m}$ beschrieben wird (Höhe: 6000 m über dem Teleskop!).

- Die individuellen Fried-Parameter $r_{0, \text { unten }}=28 \mathrm{~cm}$ und $r_{0, \text { oben }}=50 \mathrm{~cm}$ der beiden Schichten charakterisieren die Turbulenzstärke bei der Erzeugung der Phasenschirme ${ }^{2}$. Modellierte man die gesamte Turbulenz der Atmosphäre mit einer einzigen Schicht, entspräche der Wert dem eigentlichen $r_{0}$ der gesamten Atmosphäre. In unserem Fall hängen die beiden Werte jedoch mit dem effektiven Fried-Parameter über

$$
r_{0, \text { eff }}=\left[r_{0, \text { unten }}{ }^{-5 / 3}+r_{0, \text { oben }}{ }^{-5 / 3}\right]^{-3 / 5}
$$

zusammen und führen auf einen Wert von $r_{0, \mathrm{eff}}=23 \mathrm{~cm}$.

- Als 'outer scale' wurde für teleskopnahe Turbulenz $6 \mathrm{~m}$ angesetzt, für die obere Turbulenzschicht $50 \mathrm{~m}$. Diese recht konservativen Werte sind einerseits durch Gründe der Effizienz bei der Berechnung der Phasenschirme motiviert, spielen aber nach der AO-Kompensation meist keine Rolle mehr, da die 'outer scale' hauptsächlich die Amplitude der durchschnittlichen WF-Verkippung (Tip und Tilt) beeinflusst. Bei der Simulation der AO-Korrektur spielt jedoch die Amplitude der Störung keine Rolle.

\footnotetext{
${ }^{2}$ Auf die Wahl der Zahlen wird nach dieser Auflistung eingegangen.
} 
- Bezüglich der Angaben über den Wind standen lediglich meteorologische Daten für den Tag und die Stunde der Datenaufzeichnung zur Verfügung. Da dies aber meist nur einen mittleren Wert für die freie Atmosphäre liefert und nur in grober Näherung die mittleren Verhältnisse am Ort des Teleskops abschätzen kann, erachteten wir es als sinnvoller, den Windparameter in der Simulation nach Geschwindigkeit und Richtung auf Null zu setzen. Dadurch werden die zeitlich mitunter äußerst variablen Bedingungen im Mittel zumindest nicht schlechter modelliert, als durch möglicherweise falsche Abschätzungen.

- Schließlich wählten wir sieben Blickrichtungen, die in Schritten von 5"12, entsprechend den sieben Kreisringen aus Ansatz B, einen wachsenden Winkelabstand zum Lockpunkt beschreiben. Die diesen Richtungen entsprechenden Ausschnitte der Phasenschirme für die obere Turbulenzschicht werden dann in Fresnel'scher Näherung jeweils separat bis zum Teleskop hinunter propagiert und zum Phasenschirm der unteren Turbulenzschicht hinzugezählt. Für jede Richtung liegt dann also ein Phasenschirm vor, der als Eingabegröße in die AO-Simulation gefüttert werden kann.

$\mathrm{Zu}$ den individuellen Fried-Parametern ist zu sagen, dass sie einzeln nur indirekt in Erscheinung treten und im Rahmen dieser Untersuchung weitgehend Modellannahmen bilden. Die einzige bewährte Methode zur Abschätzung von $r_{0, \text { eff }}$, der Spektrale Quotient, geht von rein atmosphärischer Statistik der WF-Fehlerverteilung aus, also Abbildungen ohne AO. Uns lagen jedoch ausschließlich korrigierte Aufnahmen vor. Insbesondere kann deswegen über die teleskopnahe Turbulenz, ohne Kenntnis der AO-Aktivität, nichts direkt abgeleitet werden, denn die Korrekturen des Einflusses dieser Schicht bewirken die gleichen Verbesserungen der Abbildungsqualität in jeder Richtung des Blickfeldes. Immerhin ist ein solcher feldabhängiger Effekt für die angenommene obere Turbulenzschicht vorhanden. Allerdings müssen wir ohne zuverlässige Methoden, um aus den Bilddaten eindeutig die Höhe und Turbulenzstärke abzuleiten, für das G-Band den oben angeführten Wert von $r_{0, \text { oben }}=50 \mathrm{~cm}$ verwenden. Er soll den exzellenten Seeing-Bedingungen während der Beobachtungskampagne Rechnung tragen. Dies scheint für AO-korrigierte Aufnahmen angemessen zu sein, die eine moderate Feldabhängigkeit der Bildqualität zeigen (T. Berkefeld, priv. comm.), wie sie im vorliegenden Fall durch die Zunahme der differentiellen Bildbewegung bei wachsendem Winkelabstand zum Lockpunkt beobachtet wird. Für die anderen Wellenlängen gelten dann nach dem Skalierungsgesetz

$$
r_{0, \mathrm{eff}} \propto \lambda^{6 / 5}
$$

die entsprechenden Annahmen $r_{0, \mathrm{oben}, 4507}=53 \mathrm{~cm}$ (blaues Kontinuum), sowie $r_{0, \mathrm{oben}, 6020}=$ $75 \mathrm{~cm}$ (rotes Kontinuum).

Mangels Information über die bodennahe Turbulenzstärke übernahmen wir notgedrungen die im äußersten Kreisring des FOV gemessenen Fried-Parameter (siehe Tab. 7.2) als Werte für $r_{0, \text { eff }}$, in der (etwas gewagten) Annahme, dass sich dort zwei Effekte der AO-Korrektur gegenseitig aufheben und zu quasi-atmosphärischen Bedingungen führen, die eine Bestimmung von $r_{0, \text { eff }}$ wie im unkorrigierten Fall durch den Spektralen Quotienten erlauben: Der eine Effekt ist die unbekannte Korrektur der bodennahen Schicht, die auch im Außenbereich der Bilder wirksam ist. Der andere ist nun die Dekorrelation des 


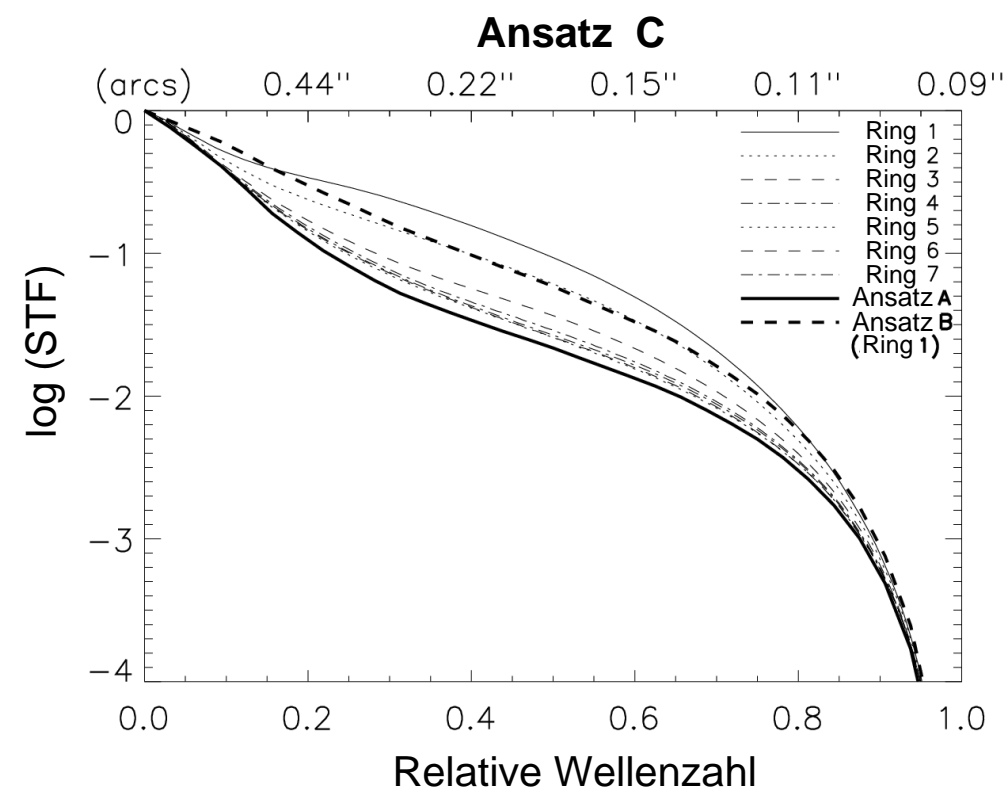

Abbildung 7.17: Logarithmische Darstellung der aus der AO-Simulation neu berechneten Modell-STFs, die im Ansatz C Anwendung finden, aufgetragen über der auf das Beugungslimit $D / \lambda$ normierten Winkelfrequenz bzw. den entsprechenden Winkeln (Obere Abszissenteilung). Zum besseren Vergleich ist hier die in Ansatz B am Lockpunkt verwendete STF mit eingetragen.

tatsächlichen Wellenfrontausschnittes der oberen Turbulenzschicht und des gemessenen Ausschnittes in Lockpunktrichtung. Durch diesen Effekt führt die AO-Korrektur deshalb einen Fehler in die effektive WF ein, der in unserer Annahme die Korrektur der unteren Schicht aufwiegt. Es wird also eigentlich immer der Wert für die untere Turbulenzschicht mit Gleichung 7.6 errechnet und wir verbleiben im G-Band mit dem oben angegebenen Wert von $28 \mathrm{~cm}$. Mit Gl. 7.7 folgen $40 \mathrm{~cm}$ im blauen Kontinuum und $54 \mathrm{~cm}$ im roten Kontinuum.

Berechnung der neuen STFs Zur Modellierung der AO-Korrektur der Wellenfronten wurde nach dem Muster der ursprünglichen Simulation sowohl die Pupillenform berücksichtigt, in diesem Fall eine kreisförmige Öffnung von einem Meter Durchmesser ohne Obstruktionen, als auch die hexagonale Anordnung der 36 Subaperturen des verwendeten Shack-Hartmann WFS und das Muster der 35 Steuerelektroden des deformierbaren Spiegels. Der Übergang von den VTT-Simulationen besteht also essentiell in der Verwendung einer idealen Pupille und einer gewissen Skalierung, die durch den größeren Durchmesser des Teleskops verursacht ist. Die Anzahl der Freiheitsgrade für die mögliche Korrektur sind bei beiden AO-Systemen gleich. Als Korrekturniveau nahmen wir jedoch nur das zweitbeste Niveau an. Es sollte vermitteln zwischen einerseits der Verwendung des bestkorrigierten Bursts und andererseits dem idealisierten Modell der Simulation, wo weder auf Wind oder zeitliche Dekorrelationen eingegangen, noch irgendwelches Rauschen berücksichtigt wurde.

Nach der AO-Korrektur bleibt aus den ursprünglichen 100 unabhängigen Wellenfront- 
realisationen jeweils eine ebensolange Serie von korrigierten Wellenfronten für jede Parameterkombination, in unserem Falle nur die Blickrichtung. Diese Serien modellieren die einzelnen Kurzzeitbelichtungen des analysierten Speckle-Bursts und haben die gleichen statistischen Mittelwerte für die Wellenfrontfehler. Die entsprechenden momentanen OTFs werden natürlich nicht über eine Autokorrelation dieser residuellen Wellenfronten berechnet, sondern können nach dem Satz von Wiener-Khinchine (siehe Anhang, Gl. B.5) über den 'Umweg' der momentanen PSF mittels FFT (siehe Anhang A) berechnet werden. Aus den OTFs folgt die STF als Mittelwert der quadrierten Absolutwerte nach Gleichung 7.2 .

Die Simulationen wurden mit den drei Wellenlängen der Untersuchung durchgeführt, sodass auch für diese Beobachtungsdaten für die gleichen sieben Regionen wie in Ansatz B individuelle STFs vorliegen. Beispielhaft ist das Set von AO-STFs für den Fall des GBandes in Abb. 7.17 dargestellt, analog zu Abb. 7.16, die die Funktionen aus Ansatz B zeigt. Die Unterschiede zwischen den in den verschiedenen Ansätzen eingesetzten STFs und den entsprechenden Resultaten wird im Folgenden diskutiert.

\subsubsection{Unterschiede in den Rekonstruktionen}

In der Beschreibung der Ergebnisse dieser Untersuchung beschränken wir uns, was die Darstellung betrifft, auf den in Abb. 7.14 mit ' 2 ' bezeichneten Ausschnitt. Er umfasst einen radial vom Bildmittelpunkt bis nach außen reichenden Bereich und zeigt somit Teile aus jedem der Kreisringe, einschließlich der Lockpunktregion, und kann zur Analyse der Winkelabhängigkeit herangezogen werden. Aus den nach den drei verschiedenen Ansätzen rekonstruierten Bildern der aktiven Region NOAA AR0636 ist in Abb. 7.18 zum direkten Vergleich der Intensitäten jeweils Ausschnitt 2 abgebildet. Offenbar liefern die drei Methoden ähnliche Resultate, denn die Position und Gestalt aller Bildstrukturen sind in allen Bildern gleich. Dies ist nicht weiter verwunderlich, wird doch in der Rekonstruktion der Fourierphasen nichts verändert, da der Einsatz einer konventionellen AO diese nicht beeinflusst.

Was die Analyse liefern soll, sind vielmehr für jeden Kreisring die Unterschiede in den relativen Helligkeiten bzw. im Kontrast, und dies bei verschiedenen Strukturgrößen, um die Effekte der nur partiellen Korrektur untersuchen zu können. Deshalb ist in Abb. 7.19 für jedes Paar von rekonstruierten Bildern ihre Differenz gezeigt.

\subsubsection{B-A}

Im oberen Feld der Abb. 7.19 zeigt das Differenzbild aus Rekonstruktion B und A den Effekt, den die Verwendung verschiedener, radial nach außen abfallender Werte für den Fried-Parameter auf die Intensitätsverteilung hat. Sowohl im Zentralgebiet als auch in den Außenregionen des FOV sind deutliche Unterschiede sichtbar. Nur bei Kreisring 4, wo der Fried-Parameter aus Methode B sehr ähnlich dem Durchschnittswert von Methode A ist, ergeben sich folglich auch keine Unterschiede in den Rekonstruktionen. Im ganzen inneren Bereich (Ring 1 bis 3) haben wir nach Ansatz A einen höheren Kontrast. Die Intensitätsunterschiede sind bei den kleinsten Strukturen besonders ausgeprägt und betragen bis zu $|\Delta I|_{\max }=0.16 I_{\text {phot }}$. Man bemerkt nun, dass dieser Effekt am Lockpunkt am stärksten ausgeprägt ist und im Bildfeld nach außen hin abnimmt. Ab einem Winkelabstand 


\section{Ausschnitt 2}

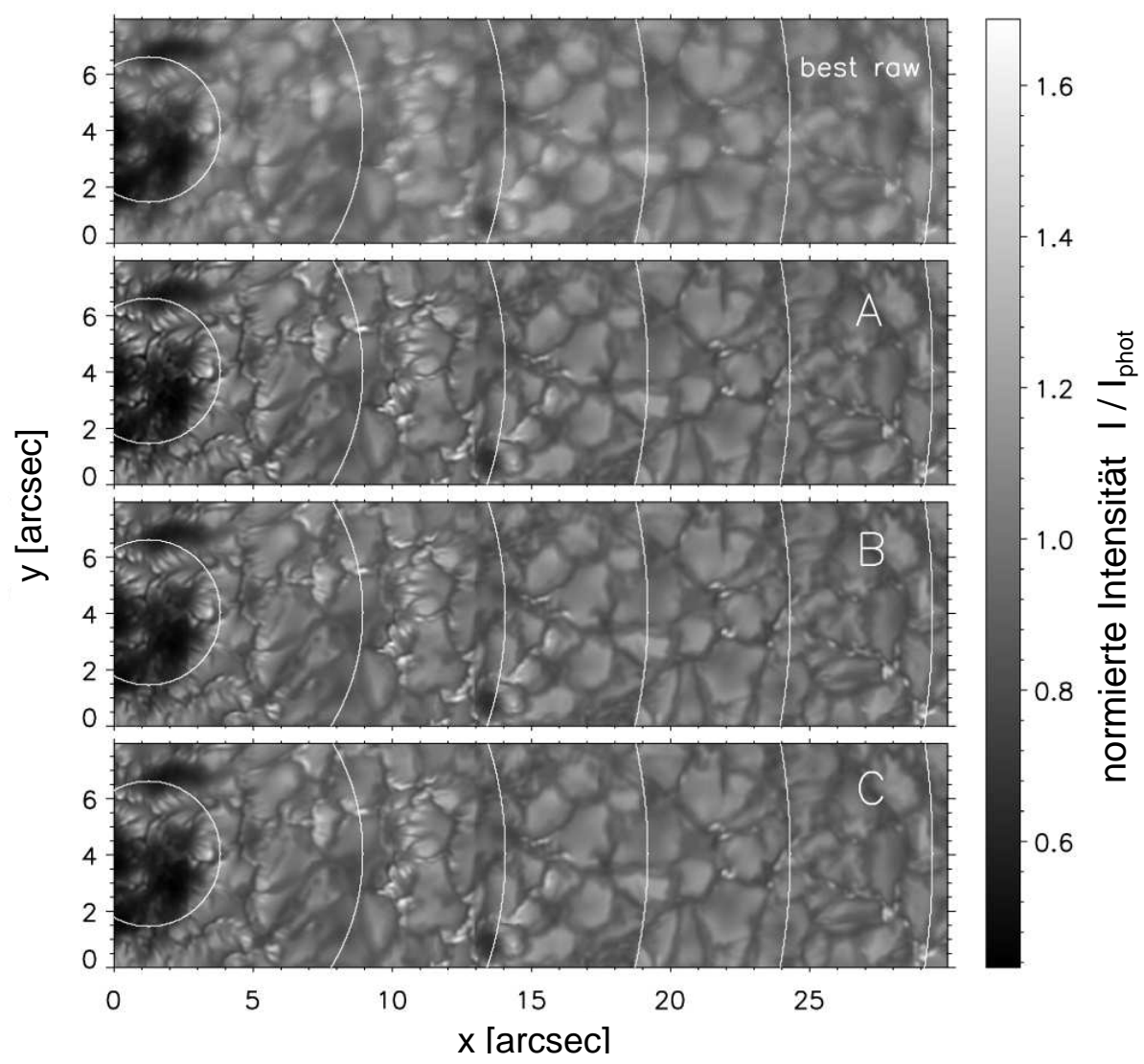

Abbildung 7.18: Unterschiede in den Resultaten der verschiedenen Ansätze werden für den gezeigten Bildbereich dargestellt (Ausschnitt 2 aus Abb.7.14). Von oben nach unten: Bestes (kontrastreichstes) Einzelbild der Aufnahmeserie ('best raw'), darunter die Rekonstruktionen nach den drei Ansätzen A, B und C. Die Bilder sind jeweils auf die mittlere Intensität der sogenannten 'ruhigen Photosphäre' normiert. Die Pore links im Ausschnitt repräsentiert etwa den Lockpunkt der AO im Zentrum des Bildfeldes, der rechte Rand des Ausschnitts fällt mit der Begrenzung des tatsächlichen Gesichtsfeldes zusammen.

von etwa $10^{\prime \prime}$ bis $15^{\prime \prime}$ verschwinden zwar die Effekte aus den oben genannten Gründen, wachsen jedoch jenseits von Ring 4 in negativer Richtung, haben also einen invertierten Effekt auf die Intensitäten. Wir beobachten also nun einen höheren Kontrast bei Ansatz B, wenn wir die äußeren Bildteile (Ring 5 bis 7) betrachten. Dort finden wir allerdings nur Differenzwerte von maximal $|\Delta I|_{\max }=0.05 I_{\text {phot }}$, wobei hier der Effekt auch für größere Strukturen verstärkt sichtbar wird. In Abb. 7.19 sind sie aus Gründen der Skalierung nur schwer zu erkennen, deshalb ist der äussere Bereich von Ausschnitt 2, also Kreisringe 4 bis 7, für alle drei Differenzbilder in Abb. 7.20 gesondert und mit eigener Intensitätsskala gezeigt.

Wir können das eben beschriebene Verhalten erklären, wenn wir dazu die entsprechenden Korff'schen Übertragungsfunktionen betrachten, die schon in Abb. 7.16 vorgestellt wurden. Dort sieht man entsprechend den obigen Ausführungen folgende Beziehungen: Ganz 


\section{Differenzbilder - Ausschnitt 2}

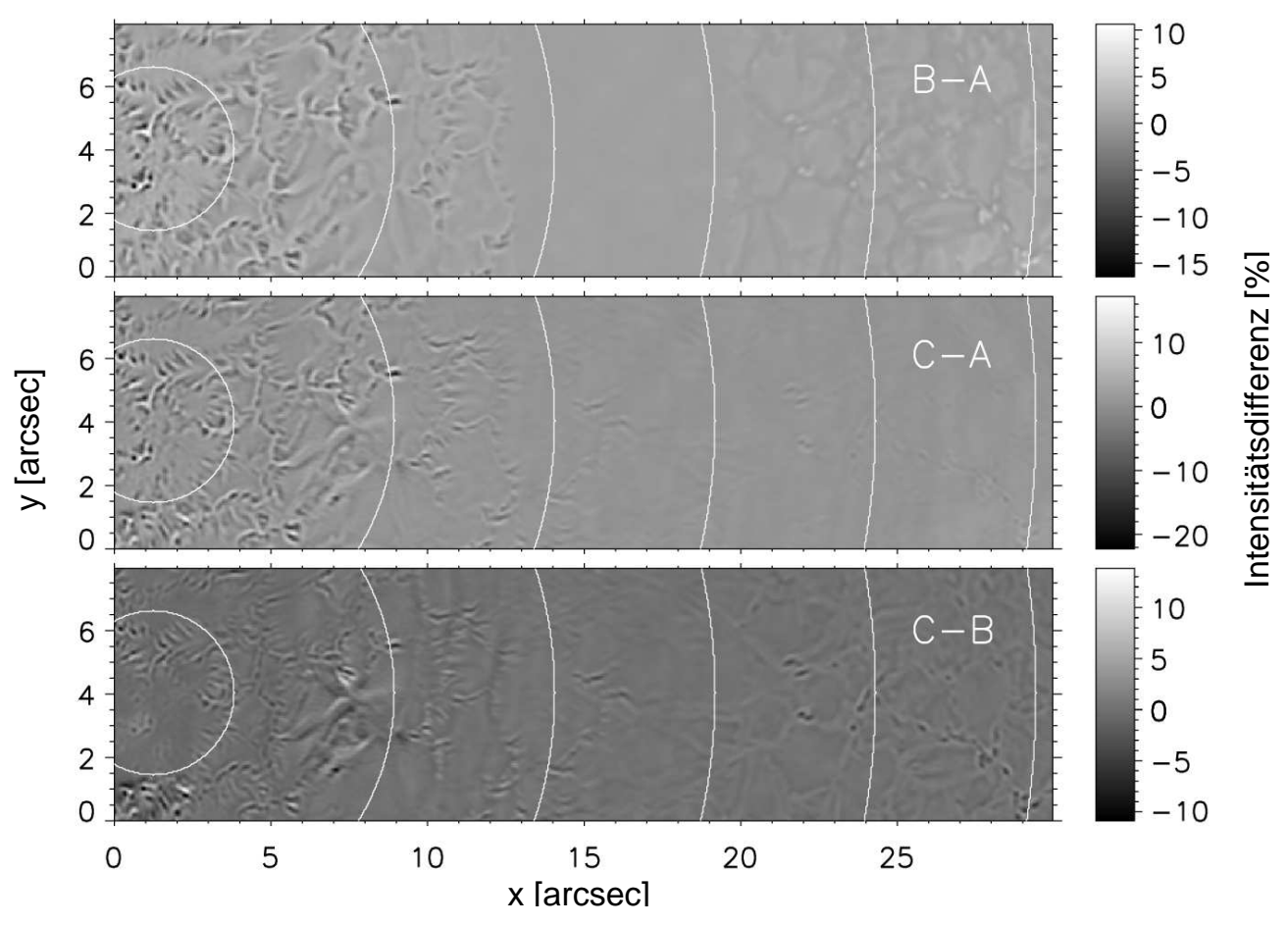

Abbildung 7.19: Differenzbilder der verschiedenen nach ihren Ansätzen benannten Rekonstruktionen aus Abb.7.18. Die Grautöne codieren die prozentuale Abweichung zweier rekonstruierter Bilder, bezogen auf die mittlere Intensität der 'ruhigen Photosphäre' $\left(I_{\text {phot }}\right)$. Von oben nach unten B-A, C-A und C-B. Die Begrenzungen durch die Kreisringe sind als weiße Konturen eingezeichnet.

allgemein führt ein größerer Fried-Parameter zu einer 'besseren' STF, d. h. einer Übertragungsfunktion mit höherem Signal. Da die Funktionen durch $r_{0}$ eindeutig bestimmt sind, gilt dies bei allen Frequenzen, sodass sich die verschiedenen Kurven auch niemals schneiden. Bei der Speckle-Bildrekonstruktion werden die Intensitätswerte aber, bis auf einen gewissen Frequenzfilter, durch Division des mittleren Leistungsspektrums der Bilder durch die STF ermittelt. So führt ein hoher Wert von $r_{0}$ über eine 'gute' STF zu einer nur leichten Verstärkung des gemessenen Spektrums. Umgekehrt wird also schlechteres Seeing zu einer entsprechend größeren Verstärkung führen. Dabei hängen die tatsächlichen Verstärkungsfaktoren und damit der gemessene Intensitätsunterschied von den absoluten Werten der beteiligten Fried-Parameter, ihrer Differenz und von der Frequenz ab. In Ansatz A wird mit dem mittleren $r_{0}$ nun sicherlich das effektive Seeing am Lockpunkt unterschätzt. Dies muss folglich dort eine Überschätzung des Kontrastes zur Folge haben. Im äußeren Bildfeld wird dagegen $r_{0}$ von Methode B niedriger eingeschätzt und führt dort zu einer Umkehrung der Verhältnisse, wie sie für die Zentralregion beschrieben wurden. Außen zeigt also Rekonstruktion B einen im Vergleich zu A höheren Kontrast und die Differenz der Bilder liefert ein 'positives', also nicht-invertiertes Bild. 


\section{Differenzbilder - Ausschnitt 2 (Detail)}

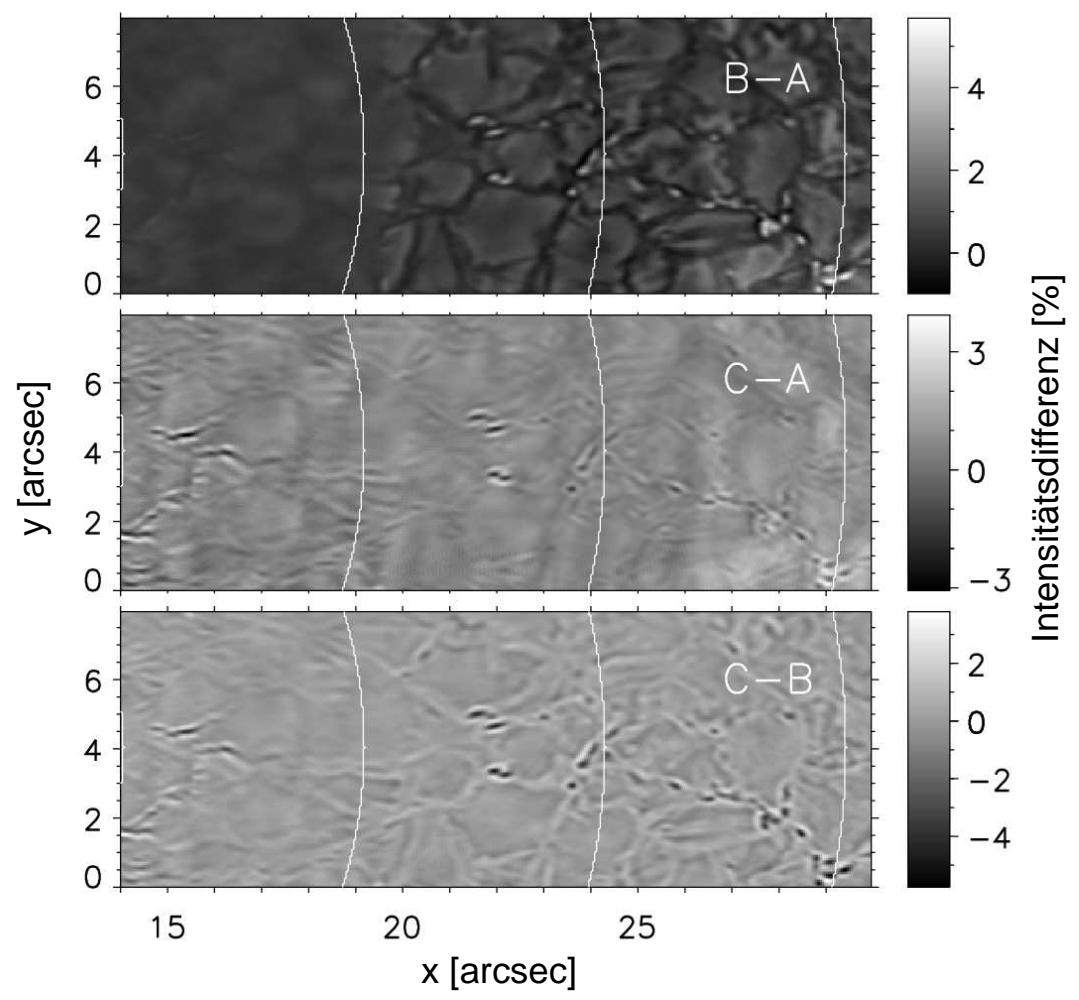

Abbildung 7.20: Äußerer Bildbereich von Abb. 7.19, hier zur deutlicheren Darstellung der Intensitätsskala, wieder als Prozentsatz bezüglich der mittleren Intensität der 'ruhigen Granulation' $\left(I_{p h o t}\right)$. Von oben nach unten sind B-A, C-A und C-B gezeigt, wobei die weißen Konturen die hier sichtbaren Teile von Kreisring 4 bis 7 trennen.

\subsubsection{C-A}

Betrachten wir nun die Unterschiede in den Intensitäten der Bilder, wie sie im Vergleich der herkömmlichen Amplitudenverstärkung mit den neuen Simulationsergebnissen sichtbar werden. Dazu sei auf die mittleren Panelen der Abbildungen 7.19 und 7.20 mit der Bezeichnung 'C-A' verwiesen. Hier beobachten wir in der Zentralregion um den Lockpunkt ähnliche Verhältnisse wie beim Vergleich zwischen B und A, obwohl der Betrag der Intensitätsvariationen in der Differenz C-A sowohl größer ausfällt $\left(|\Delta I|_{\max }=0.22 I_{\text {phot }}\right)$ als auch bis zu größeren Winkelabständen $\left(15^{\prime \prime}\right.$ bis $\left.20^{\prime \prime}\right)$ signifikante Unterschiede in den Resultaten zeigt, wobei wiederum Unterschiede auf den kleinsten räumlichen Skalen am stärksten ausgeprägt sind. Gehen wir bei Ansatz A von einer übermäßigen Verstärkung der Intensitäten am Lockpunkt aus, wie oben plausibel zu machen versucht wurde, unterdrückt Methode $\mathrm{C}$ dort diese Überrekonstruktion noch besser als Ansatz B.

Nach außen hin finden wir, im Gegensatz zu B-A, keine Invertierung beim Differenzbild, sieht man von den als dunkle Punkte sichtbaren intergranularen Elementen ab. Betrachtet man die Skala der Intensitätsunterschiede von $\pm 3 \%$ und bedenkt, dass die Genauigkeit der Speckle-Rekonstruktion bei ähnlichen Werten liegt, können wir sagen, dass hier in den 
äußeren Bildbereichen vom praktischen Standpunkt aus betrachtet keine AO-Korrektur mehr zu bemerken ist. Die Rekonstruktion nach Ansatz C zeigt dort folglich eine sehr ähnliche Verstärkung wie das Ergebnis nach Ansatz A.

Werfen wir wiederum einen Blick auf die zugehörigen STFs, die in Abb. 7.17 gegen die relative Wellenzahl aufgetragen sind. Für die STFs am Lockpunkt gilt die gleiche Argumentation wie für die Differenz B-A. Darüberhinaus bemerkt man, dass alle STFs, die in Rekonstruktion C verwendet werden, oberhalb der STF aus Ansatz A liegen. Dies stimmt überein mit der diskutierten Beobachtung, dass keine Invertierung des Bildes sichtbar wird und die Verstärkungsfunktion bei Ansatz C sowohl über das Bildfeld hinweg, als auch für fast alle Frequenzen niedriger ausfällt. Nur in den höchsten Raumfrequenzen wird ein abweichendes Verhalten bemerkbar. Dieser Bereich wird aber schon stark von eventuellem Rauschen in den Daten beeinflusst und muss für genauere Aussagen gesondert modelliert werden.

\subsubsection{C-B}

Der unterste Bildstreifen in Abb. 7.19 präsentiert das Differenzbild aus den Resultaten der beiden neuen Methoden, C-B. Natürlich kann diese Differenz nichts Neues zeigen, sondern nur die Kombination der beiden schon diskutierten Fälle. Da aber noch entschieden werden soll, welche der neuen Methoden realistischere Ergebnisse liefert, lohnt sich immerhin die Beachtung der Werte für die Intensitäten. Um den Lockpunkt werden zwar in zwei sehr kleinen Regionen im zweiten Kreisring Unterschiede von bis zu 13\% gemessen, es hat jedoch den Anschein, als hätte dies seine Ursache in Ungenauigkeiten beim Zusammensetzen der einzelnen Teilbilder. Im gesamten übrigen FOV, eingeschlossen die äußeren Kreisringe, übersteigen die Unterschiede $|\Delta I|_{\max }=0.06 I_{\text {phot }}$ nicht. Wie schon erwähnt, werden nach Ansatz $\mathrm{C}$ im gesamten Bildfeld die rekonstruierten Intensitäten kleinerer Strukturen weniger betont als nach Ansatz B, jedoch sehr ähnlich, was Strukturen der Größe etwa von Granulen betrifft. In diesem Effekt scheint der andersartige Verlauf der STF-Kurven aus der Simulation durch, d. h. eine frequenzabhängige Veränderung der Verstärkungsfunktion, wie sie von den konventionellen STFs her nicht bekannt ist.

\subsubsection{Winkelabhängigkeit des Kontrastes}

Um nun ein zusätzliches, unabhängiges Maß für die Qualität jeder Rekonstruktion zu erhalten, untersuchten wir den $r m s$-Kontrast der resultierenden Bilder und analysierten seine Feldabhängigkeit. Das Ergebnis legt dann die Güte der Rekonstruktionsmethode fest, sollte das Beobachtungsobjekt doch, bei idealer Abbildung, überall im Bildfeld die gleichen Kontrastwerte zeigen, den gleichen Bildstrukturgehalt vorausgesetzt.

Wir berechneten die rms-Werte des Kontrastes in jedem Kreisring separat. Dabei gingen nur solche Pixel in die Rechnung ein, die innerhalb der eingezeichneten Kontourlinien der Ringe liegen. Allerdings ergibt sich hier eine Schwierigkeit bei der Auswahl der Grundgesamtheit. Für eine aktive Region mit sehr dunklen und sehr hellen sichtbaren Einzelheiten wird der berechnete rms-Wert außerordentlich hoch, ungeachtet der Bildrekonstruktionsmethode. Dies ist für einen Bildausschnitt, der über das ganze Bildfeld hinweg ähnliche Strukturen zeigt, nicht weiter hinderlich. In unserer Situation haben wir es aber mit sehr 


\section{Feldabhängigkeit des Kontrastes}

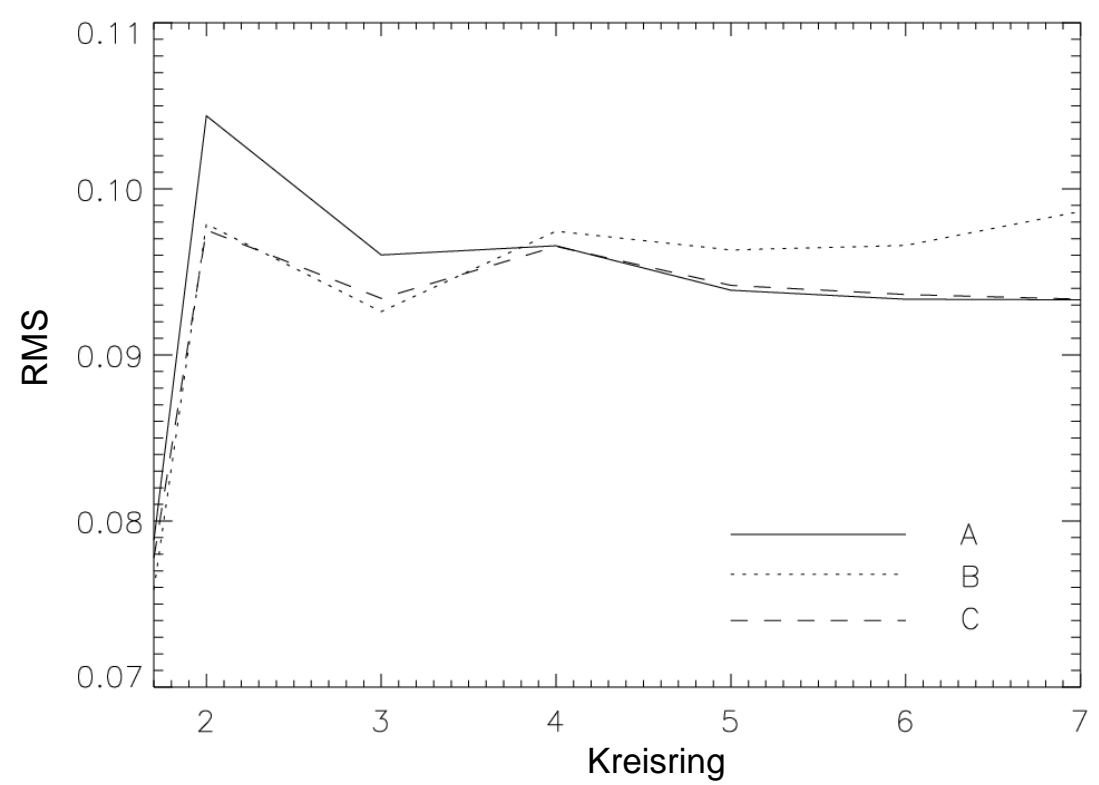

Abbildung 7.21: Feldabhängigkeit des $r m s$-Kontrastes für die drei SpeckleRekonstruktionen nach Ansatz A, B und C. Die Bilder wurden zur Berechnung auf die mittlere Intensität der 'ruhigen Photosphäre' normiert. Da der Kontrast der aktiven Regionen aber vorwiegend den Strukturgehalt der Bildausschnitte widerspiegelt, wurden bei dieser Rechnung Bereiche ausgelassen, die durch Poren oder andere Strukturen bedeckt waren, die mit starken magnetischen Phänomenen in Zusammenhang gebracht werden. Zu Letzteren zählen haupsächlich intergranulare 'bright points' und filamentartige Strukturen. Dies sollte für jeden Ring jeweils die 'ruhige Photosphäre' repräsentieren. Als Folge davon besitzen wir jedoch keinen aussagekräftigen Wert für die Lockpunktregion, da sie aus einem Teilbild besteht, das bis auf wenige Pixel von einer Pore bedeckt wird.

unterschiedlichen Strukturen zu tun, die unregelmäßig über die Ringe verteilt sind. Deren Kontrastwerte weisen in kleinen Ausschnitten sehr große Abweichungen zum Mittlerwert eines ganzen Kreisringes auf. Kleine Unterschiede im Kontrast, wie sie durch unsere verschiedenen STFs auftreten, lassen sich auf diese Weise nicht erkennen. Die errechneten Werte würden gewissermaßen den Informationsgehalt der Bilder kodieren, nicht aber die Rekonstruktionsmethode bewerten können. Was wir hier benötigten, wären Daten, die ein Beobachtungsobjekt homogeneren Kontrasts dokumentieren, beispielsweise solare Granulation weitab von aktiven Gebieten ('ruhige Sonne').

Nichtsdestoweniger haben wir versucht, solch eine 'ruhige Photosphäre' nachzuahmen, indem wir die beiden folgenden Einschränkungen bei der Auswahl der in die rms-Berechnung eingehenden Pixel gelten ließen:

- Wir definierten eine unterer Intensitätsgrenze von $75 \%$ der mittleren Intensität eines nicht-aktiven Gebietes im Bildfeld, um die dunklen Gebiete der Pore am Lockpunkt und der Poren im Bildfeld rechts unten auszuschließen. 
- Ausßerdem versuchten wir durch eine manuelle, sukzessive Absenkung der zugelassenen oberen Intensität, helle Strukturen zu eliminieren, die gewöhnlich durch magnetische Aktivität hervorgerufen werden, nämlich die intergranularen 'bright points'.

Zur Festlegung der oberen Grenze für die Intensitätwerte zulässiger Pixel wurde iterativ das hellste Pixel gesucht und, zusammen mit einer quadratischen Umgebung von \pm 3 Pixeln in jeder Richtung, aus der Menge der Pixel im Kreisring ausgenommen. Nach einer gewissen Anzahl von Wiederholungen dieser Prozedur fing der Algorithmus an, die Positionen der Zentren heller Granulen zu liefern. Hier griffen wir manuell ein und beendeten das Verfahren.

Nur diejenige Bildregion innerhalb eines Kreisringes, die von beiden Kriterien unberührt blieb, wurde also zur Berechnung des Kontrastes herangezogen. Aus diesem Grund besitzen wir allerdings keinen aussagekräftigen Wert für den innersten Ring am Lockpunkt der AO; fast das gesamte Gebiet wird dort von der mehrmals angesprochenen Pore und hellen Signaturen magnetischer Aktivität eingenommen, sodass dort kein repräsentativer Kontrastwert gewonnen werden konnte. Die Graphen, die wir dadurch aus den drei Rekonstruktionen erhalten haben, bestätigen jedoch recht genau unsere Diskussion der Differenzbilder im vorhergehenden Abschnitt, und sind zum direkten Vergleich in Abb. 7.21 aufgetragen.

Unsere neue Methode der STF-Berechnung führt also über das Blickfeld zur gleichmäBigsten Bildqualität im Sinne des $r m s$-Kontrastes. Dies rechtfertigt unseren Ansatz, den Einsatz der Adaptiven Optik zusammen mit genaueren Modellen der Atmosphäre in die Berechnung neuer Übertragungsfunktionen miteinzubeziehen, um aus dem Datenmaterial zuverlässigere Informationen mit dem Rekonstruktionsalgorithmus zu gewinnen.

\subsubsection{Weitere Anmerkungen zur Analyse}

In der Modellierung anisoplanatischer STFs müssen die Höhe und die Turbulenzstärke der oberen Turbulenzschicht bekannt sein bzw. angegeben werden. Beide Werte zusammen konnten aus der beobachteten Bildbewegung hier nicht abgeleitet werden. Eine genaue und zuverlässige Methode zu deren Bestimmung wäre durch die Messungen von zwei WFS möglich, die, je nach Justierung, die Wellenfrontdeformationen vorwiegend eines einstellbaren Höhenbereichs messen, wie es in einem Ansatz für eine Multikonjugierte Adaptive Optik (MCAO) getan wird (von der Lühe et al. 2005, Berkefeld 2005). Denkbar wäre auch ein größeres, anisoplanares Gesichtsfeld für die Subaperturen eines einzelnen WFS, wodurch die Wellenfronten in verschiedenen Richtungen bestimmbar wären. Korrelationen der errechneten Deformationen gäben dann Aufschluss über die Höhe der Schicht. Diese Ansätze werden jedoch noch näher zu untersuchen sein und müssen ihre Berechtigung mit realen Daten erst unter Beweis stellen. Sie stellen also einen Ausblick dar.

Anders als bei dieser Untersuchung ist für die Abschätzung des tatsächlichen FriedParameters aus Daten mit AO-Einfluss die Sammlung von Daten der AO-Aktivität während der Beobachtungszeiten angezeigt. Dabei sind am wichtigsten die Verschiebungswerte des WFS, die Aktuatorsignale des DM, die Rekonstruktionsmatritzen und die exakte Zeit der Aufnahme. Aus diesen Werten lässt sich dann das ursprüngliche, atmosphärische $r_{0}$ etwa über die Varianz einzelner Moden rekonstruieren. 
In Zusammenhang damit sei nocheinmal darauf hingewiesen, dass man mit der Methode des Spektralen Quotienten (von der Lühe 1984) die Parameter der atmosphärischen Turbulenzstärke und die der Korrekturstärke der AO nicht klar unterscheiden kann, da sie im Effekt interferieren. Deshalb ist für eine präzise Simulation von STFs einer Beobachtungsreihe möglichst auch das Korrekturniveau bzw. die Anzahl der korrigierten ZernikeModen mitzunotieren. Im Falle der vorgestellten Daten war diese Information nicht zugänglich. Allerdings herrschten während der Aufnahmen sehr gute Seeing-Bedingungen, und von den exzellenten Daten wurde wiederum die beste Speckle-Serie für die Analyse ausgesucht. Wir gehen deshalb davon aus, dass eine konstante und optimale WFKompensation gewährleistet war und unsere Modellannahmen bei der AO-Simulation berechtigt waren.

\subsection{Beobachtungsgestuitzte Simulation}

In diese Arbeit finden quantitative Ergebnisse zu Analysen der aufgenommenen Protokolldateien der AO-Aktivität während der Speckle-Beobachtungen aus zeitlichen Gründen leider keinen Eingang. Jedoch soll hier das Vorgehen erklärt und der erwartete Nutzen dieser Daten grob angezeigt werden.

\subsubsection{Rekonstruktionsmatrizen und Fingerabdruck der Geometrie}

Will man Simulationsergebnisse mit den experimentellen Daten vergleichen, muss die relative Lage von Lenslet-Array gegenüber dem deformierbaren Spiegel und der Pupille in der Simulation so nachgebildet werden, wie sie in der experimentellen Umgebung bei der Aufnahme der Protokolldaten eingestellt war, da sonst schon die Reihenfolge der Daten, etwa der Nummerierung der Subaperturen des WFS oder der Aktuatoren des DM, nicht übereinstimmen würden und einen Vergleich unmöglich machten. In den Rekonstruktionsmatrizen aus der Kalibrationsprozedur ist nun gewissermaßen der Fingerabdruck der geometrischen Anordnung gespeichert, sodass wir, wie schon in Abbildung 5.6 am Fall einer Optimierung gezeigt wurde, hier ebenso mit den experimentellen Matrizen verfahren können. Diese konnten mit einer Reihe Matrizen aus der Simulation verglichen werden, die für jeweils eine Kombination der betreffenden Parameter berechnet wurden. Dazu habe ich den Korrelationskoeffizient der experimentellen und jeweils einer Matrix aus der Simulation gebildet, also der Wert am Ursprung der Kreuzkovarianzfunktion. Auf diese Weise konnte der Versatz in Pixel $(\Delta x=5$ und $\Delta y=1)$ und die Verdrehung des TT-Spiegels $\left(\Delta \rho=91^{\circ}\right)$ und des DM $\left(\Delta \rho=55^{\circ}\right)$ bestimmt werden. Dies muss beim Übergang von der experimentellen Umgebung bei KAOS am VTT zur Analyseumgebung am Rechner in der Simulation berücksichtigt werden.

Erst mit dieser Einstellung der Wertekombination im Simulations-Code lassen sich die entsprechenden simulierten und experimentellen Daten der Anordnung nach vergleichen.

\subsubsection{Parameter aus der AO-Aktivität}

Aus den protokollierten Werten der AO-Aktivität lassen sich Aussagen über atmosphärische und technische Parameter, wie Turbulenzstärke und Kompensationsleistung machen, 


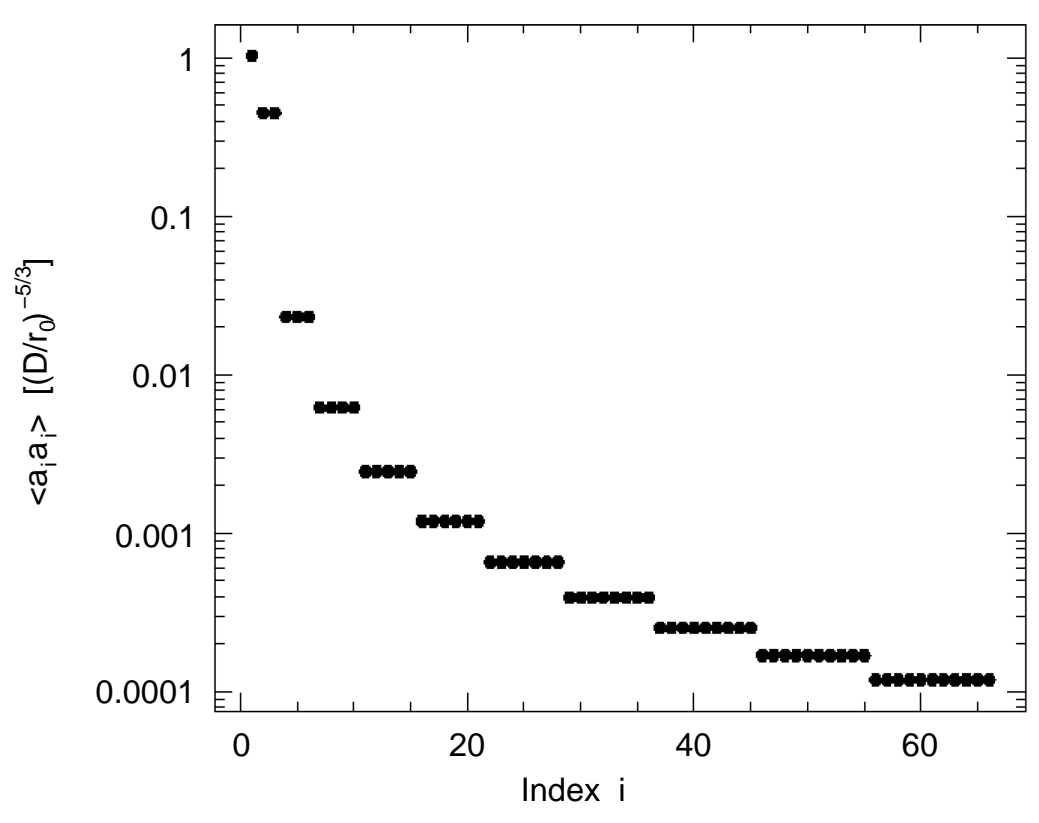

Abbildung 7.22: Normierte Beiträge der Koeffizienten bei einer Zernike-Entwicklung der mittleren atmosphärischen Wellenfrontfehler (bei Kolmogorov'scher Statistik) bis zum radialen Grad $n=10$. Eine solche Darstellung wird ebenso für die korrigierten und die residuellen Wellenfrontfehler von experimentellen Daten wie auch der Simulation benutzt werden. Man sieht in der theoretischen Verteilung auch sehr deutlich, wie der rms-Wert des Wellenfrontfehlers trotz schnell steigender Anzahl von korrigierten Moden immer langsamer abnimmt.

die als Schätzwerte in die Modellierung der Simulation eingehen können, um PSFs oder STFs zu möglichst ähnlichen Bedingungen, wie zum Zeitpunkt der Beobachtungen, errechnen zu können. Dazu sei kurz aufgeführt, wie Schätzwerte für die Turbulenzstärke und Kompensationsleistung aus den vier Größen Shift-Werte, Aktuator-Signale, Rekonstruktionsmatrizen und Zernike-Transformatormatrix abgeleitet werden können. Dabei unterscheiden wir nach Aufnahmemodus, also ob mit (aktiv) oder ohne (passiv) AO-Unterstützung beobachtet wird:

Offener Regelkreis (passiv):

\section{- Atmosphärische Aberrationen}

Dazu sind nur die mitgeschriebenen Zeitreihen der Shift-Werte des WFS auszuwerten. Mit der Zernike-Transformatormatrix wird, jeweils durch eine Multiplikation mit dem Datenvektor einer einzelnen WF-Messung, die entsprechende Zerlegung der WF in Zernike-Moden erhalten. Durch einen Vergleich der zeitlich gemittelten Verteilung mit der theoretischen Verteilung (siehe Abb.7.22) kann über die absolute Amplitude der Moden die Turbulenzstärke, also der Fried-Parameter $r_{0, \text { eff }}$ abgeleitet werden. Dieser Wert ist für die Simulation sehr wichtig, da damit die Modellierung der Atmosphäre weitgehend bestimmt wird. Natürlich ist die Analyse nur im Bereich der Messgenauigkeit des WFS mit seiner festen Subaperturgröße möglich. 
Kleinskaligere WF-Deformationen, als durch den Abstand der Linsen festgelegt, können selbstverständlich nicht gemessen werden.

\section{- Statische Aberrationen des Teleskops}

Aus den relativen Abweichungen der mittleren Amplituden der einzelnen ZernikeModen kann, unter der Voraussetzung etwa Kolmogorov'scher Statistik der Wellenfrontfehler, auf statische WF-Fehler geschlossen werden, die im optischen Aufbau vorliegen. Diese können dann ebenfalls in die Simulationen mit einbezogen werden bzw. bei anderen Analysen abgezogen werden, wo man an rein atmosphärischen Größen interessiert ist.

Geschlossener Regelkreis (aktiv):

\section{- Residuelle Wellenfront}

Da der WFS bei laufender Korrektur jeweils nur die Differenz aus aktueller Wellenfront und Spiegelform aus dem letzten Kontroll-Zyklus misst, bieten die Verschiebungswerte des Sensors in diesem Fall eine Abschätzung der oberen Grenze für den Wellenfrontfehler (am Lockpunkt), der in den Daten wirksam ist, und damit auch eine Schätzung der Strehl-Zahl, um die Abbildungsqualität zu beschreiben.

\section{- Korrigierte Zernike-Moden}

In ähnlicher Weise wie bei den residuellen Signalen des WFS lassen sich aus den Aktuatorsignalen allein die tatsächlichen Korrekturen ablesen. Praktisch werden aus den Aktuatorsignalen mit Hilfe der Interaktions-Matrix (deren Pseudo-Inverse die Rekonstruktor-Matrix ist) Shift-Werte errechnet, die dann wieder in eine ZernikeEntwicklung übersetzt werden können. Auch hier ergibt sich eine Abschätzung der Korrekturqualität über den entsprechenden $r m s$-Wert.

\section{- Atmosphärische Störung (Low-Pass)}

Aus der Kombination der WFS-Daten und der Aktuator-Signale kann auch, im Rahmen der Messgenauigkeit, auf die atmosphärische Wellenfront geschlossen werden. Dazu müssen allerdings noch offset-Werte bekannt sein, um auf absolute Verschiebungen bzw. die absolute Spiegeldeformation zurückrechnen zu können. Ansonsten müssen nur wieder die Aktuatorwerte in Verschiebungen am WFS-CCD-Chip umgerechnet werden und anschließend zu den WFS-Shifts addiert werden.

Diese Messung ist in etwa gleichwertig mit den Shift-Werten vom offenen Regelkreis, jedoch wird das Ergebnis hier zusätzlich verschlechtert, da die Spiegeldeformation nicht exakt aus WFS-Daten rekonstruierbar ist.

\subsubsection{Anisoplanatische PSFs aus den Speckle-Daten}

Zur Untersuchung der Winkelabhängigkeit der WF-Fehler wäre natürlich am hilfreichsten, WF-Messungen eines zweiten WFS zur Verfügung zu haben, der, wie bei der MCAOErweiterung von KAOS, konjugiert zur oberen Turbulenzschicht der Atmosphäre angeordnet liegt und direkt eine Trennung der Turbulenzanteile zuließe. Eine andere Möglichkeit wäre eine Messung der Szintillation, die ebenfalls zur Trennung der Turbulenzanteile der beiden Schichten dienen kann (Berkefeld 1998). Dieses Konzept müsste aber zunächst auf die WF-Messung bei der Sonnenbeobachtung übertragen werden, da die Photosphäre 


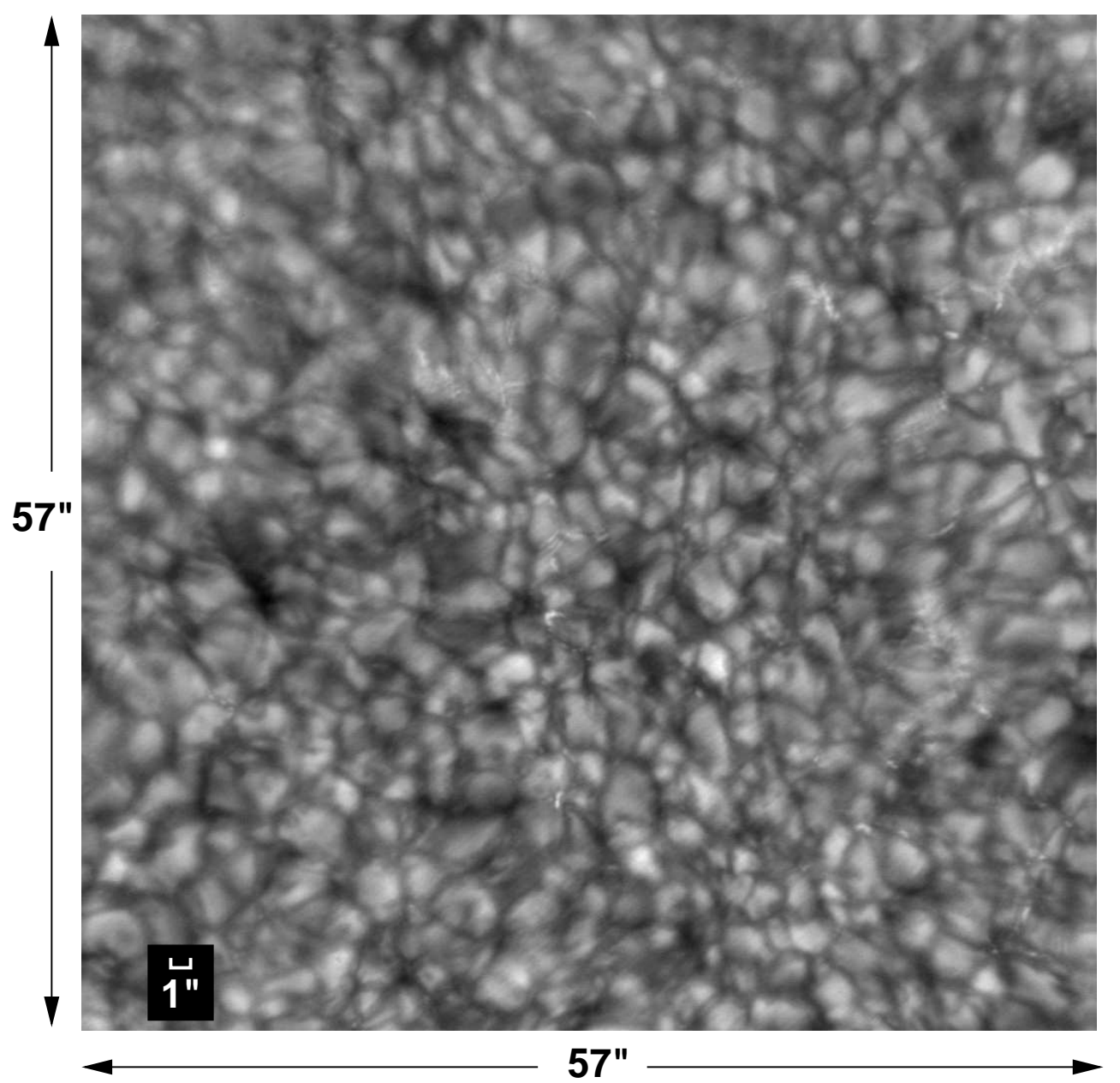

Abbildung 7.23: Typisches Speckle-Bild der Granulation der solaren Photosphäre. Die Kurzzeitbelichtung $(10 \mathrm{~ms})$ zeigt deutlich den Anisoplanatismus, der durch die AOWellenfrontkorrektur sichtbar wird: Im Zentrum ist die Bildqualität auch im zeitlichen Mittel besser als in den äußeren Bereichen des Gesichtsfeldes. Während der Aufnahme dieses Bildes notierte der Rechner auch die Daten zur WF-Korrektur durch die AO.

als flächenhafte Lichtquelle ohne feste Strukturen mit höchst unterschiedlich hellen Details eine Unterscheidung der Szintillation von der Quellintensität sehr schwierig macht. Relativ einfach ist es jedoch, aus den Bilddaten PSFs zu isoplanaren Bereichen zu extrahieren (siehe Abbildungen 7.23 und 7.24), die im zeitlichen Mittel und je nach relativem Winkelabstand zum Lockpunkt den Anisoplanatismus deutlich widerspiegeln sollten. Hier könnte ein radialer Kompensationsabfall mit theoretisch zu erwartendem Abstandsgesetz angefittet werden und so Rückschluss auf den isoplanaren Winkel gezogen werden.

\subsubsection{Simulationsgestiutzte Beobachtung}

Aus dem Vergleich der AO-Mitschriften bei der Beobachtung mit gleichartigen Daten aus der Simulation, zusammen mit den Informationen über die anisoplanatische PSF bzw. 


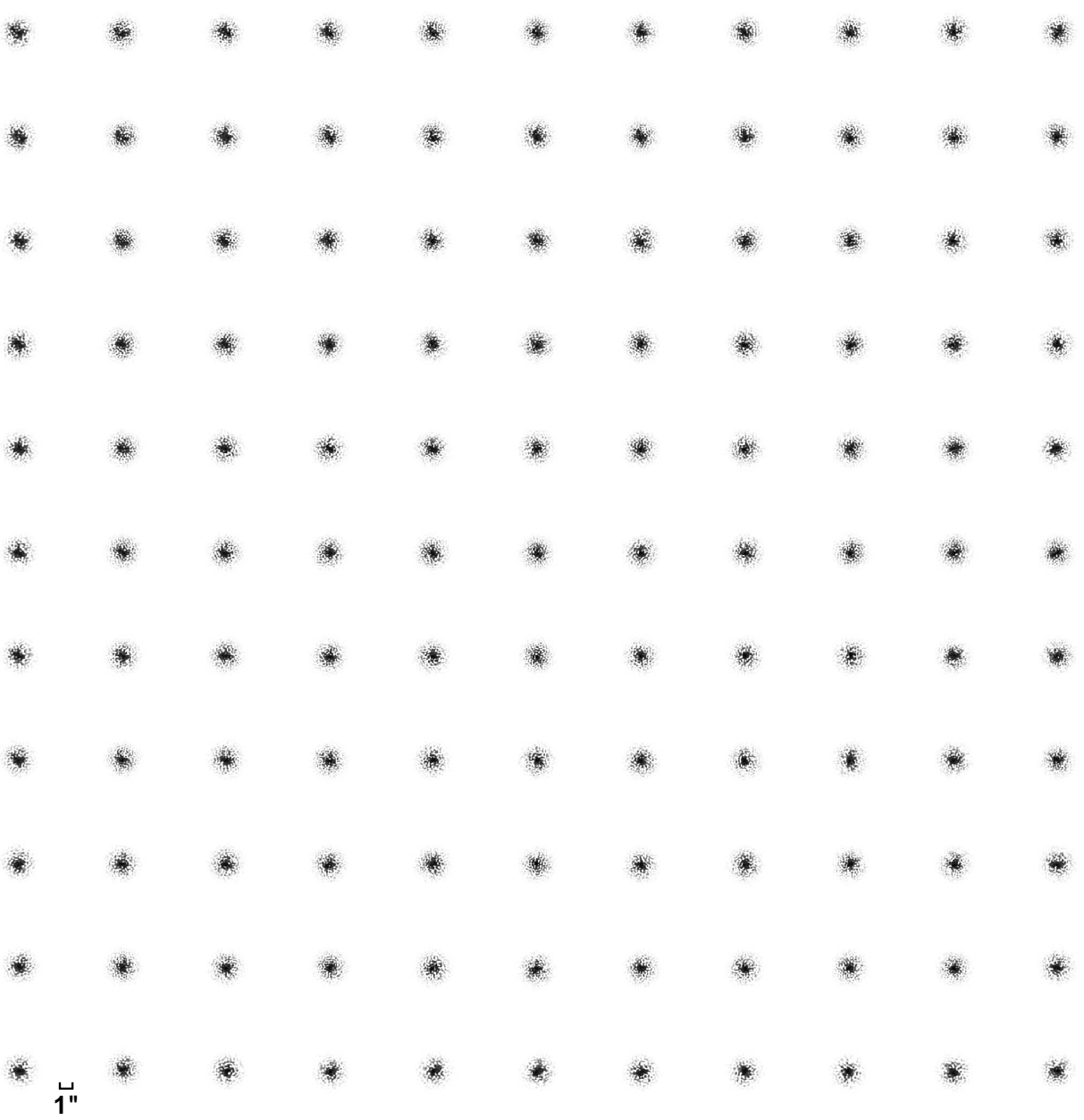

Abbildung 7.24: Aus den Bilddaten lassen sich durch iterative Methoden mit Randbedingungen geschätzte PSFs für isoplanare Bildausschnitte gewinnen (freundlicherweise zur Verfügung gestellt von F. Wöger, KIS), hier am Beispiel von $11 \times 11$ sich überlappenden Ausschnitte des Bildes aus Abb. 7.23. Zusammen mit den zeitgleich notierten Wellenfrontdaten und Aktuatorsignalen wird versucht, die Turbulenzverhältnisse in der Atmosphäre und technische Parameter abzuleiten. Aus vielen solchen Aufnahmen kann dann eine gute individuelle Schätzung der STF für alle dargestellten Subfelder gewonnen werden, die zuverlässigere Intensitäten bei der Speckle-Rekonstruktionen gewähren.

Winkelabhängigkeit der AO-Korrektur aus den Bilddaten selbst, sollte es möglich sein, eine, wenn auch nicht exakte Bestimmung, so doch eine eindeutige Eingrenzung der 'GröBenordnung' der verwendeten Modellparameter herauszufinden, sodass eine gut auf die Beobachtungen abgestimmte Modell-Übertragungsfunktion für eine weitere Rekonstruktion der Bilddaten bestimmt werden kann. 


\section{Zusammenfassung und Ausblick}

Den Rahmen für diese Arbeit bildet das Problem der Bestimmung und/oder Abschätzung der anisoplanatischen und zeitabhängigen Übertragungseigenschaften eines Teleskops bei Einsatz einer Adaptiven Optik. Lösungen in diesem Bereich sind gerade für die photometrische Genauigkeit von Speckle-rekonstruierten Bildern von großer Bedeutung (Puschmann \& Sailer 2006, Mikurda et al. 2006). Aus dem weiten Feld möglicher Ansätze und Untersuchungen zur Analyse habe ich in einem numerischen Zugang Übertragungsfunktionen mit Hilfe einer Simulation berechnet, die die Wellenfrontpropagation durch Atmosphäre und Teleskop mitsamt der AO-Module berücksichtigt. Dabei wurde der Einfluss der Atmosphäre mit zwei Turbulenzschichten modelliert, um die Richtungsabhängigkeit der Wellenfrontkorrektur untersuchen zu können. Eine Parameterstudie mit einer Reihe weiterer Variablen, wie der Korrekturempfindlichkeit, der Turbulenzstärke in den einzelnen Schichten und Windstärke mit Windrichtung in der oberen und unteren Atmosphärenschicht, zeigte den Charakter und die Größe der Veränderungen in der theoretisch zu erwartenden Bildqualität, gemessen an der Strehl'schen Definitionshelligkeit.

Die Anwendung auf eine Speckle-Rekonstruktion hochaufgelöster Beobachtungsdaten der solaren Photosphäre machte einerseits deutlich, dass die Berücksichtigung des AOEinflusses signifikante Unterschiede bei der Bewertung des Kontrastes und der relativen Intensitäten hervorruft, andererseits kann jedoch, offenbar schon ohne spezifische Kenntnis der exakten tatsächlichen AO-Aktivität während der Datenerfassung (siehe auch Cagigal \& Canales 2000a) und nur durch geschicktes Abschätzen der wahrscheinlichsten Parameter, mit den simulierten Speckle-Transferfunktionen eine deutlich homogenere Bildqualität in der rekonstruierten Szene erreicht werden. Hier bietet sich geradezu an, mit weiteren Informationen zur Wellenfrontkorrektur in die Modellierung zu gehen, um besser auf die Daten abgestimmte Parameter, sowohl bezüglich des Atmosphärenmodells, wie auch der AO-Leistungsdaten, in die Simulation eingeben zu können. Die dazu nötigen Protokoll-Dateien wurden aufgenommen, ihre Auswertung konnte hier jedoch nur kurz angerissen werden. Hier besteht noch Handlungsbedarf bei der Darstellung und Analyse, um in der Zukunft zu einer anwenderfreundlichen Implementierung der Analysen innerhalb routinemäßiger Datenreduktion zu gelangen. darzustellen und mit den beobachteten Bilddaten in Verbindung zu bringen. Ich hoffe immerhin, dieses Ziel, wenn auch nicht mehr im Rahmen dieser Arbeit, weiterverfolgen zu können.

Über die Zielsetzung dieser Arbeit hinausgehend sind mit den Simulationen und den AOProtokolldateien auch Möglichkeiten zu untersuchen, wie gewisse experimentelle Parameter optimiert werden können. Schon durch den Vergleich der Kalibrationsmatrizen konnte die tatsächliche Einstellung am Teleskop aus den Mitschriften abgelesen und in den Parameterraum der Simulation eingeordnet werden, wo die optimalen Werte bezüglich der geometrischen Anordnung einfach bestimmbar sind. Prinzipiell ist dieses Vorge- 
hen mit allen Größen möglich, die zum einen protokolliert und zugleich in der Simulation modelliert werden können.

Untersuchungen zur winkelabhängigen Korrekturleistung sind für die Entwicklung der Multi-konjugierten Adaptiven Optik (MCAO) natürlich von besonderem Interesse. Die in dieser Arbeit verwendeten Modelle der Atmosphäre und der Wellenfrontkorrektur sind im Ansatz dem bei KAOS verfolgten Konzept zur MCAO-Erweiterung verwandt. Somit ist die Erweiterung des Simulations-Codes in der gleichen Weise naheliegend.

Schließlich sollen alle diese Anstrengungen nicht zuletzt auch im Hinblick auf das neue 1,5-Meter Sonnenteleskop GREGOR auf Teneriffa unternommen werden. Dabei können selbstverständlich die hier angestellten Versuche zur besseren Modellierung der Übertragungseigenschaften ebenfalls angewendet werden, und ich hoffe, meine Arbeit kann einen wichtigen Beitrag für die Qualität sowohl der Beobachtungsdaten wie auch der daraus entstehenden Forschungsergebnisse leisten. 


\section{A Fouriertransformation}

Im Bereich der Optik stellt die Fouriertransformation $\mathcal{F}$ ein höchst wichtiges analytisches Werkzeug dar. Bilder als zweidimensionale Datenfelder physikalischer Messwerte, wie sie etwa vom CCD-Chip einer Kamera produziert werden, können normalerweise mit reellen Zahlen dargestellt werden und liefern nach Anwendung von $\mathcal{F}$ ein Hermite'sches Spektrum, das aus Symmetriegründen hohe Redundanz besitzt. Eine Halbebene im (komplexen) Frequenzraum enthält schon die gesamte Information des Spektrums. Die nur ausschnitthaft mögliche Erfassung eines Phänomens durch eine reale Messung bewirkt jedoch eine Beschränkung des gesamten Informationsgehalts, also des zeitlichen und/oder räumlichen Variablenbereichs im Ortsraum, sowie zur Reduzierung der Auflösung im Frequenzraum. Es können andererseits keine beliebig hohe Frequenzen registriert werden, sodass eine gewisse Grenzfrequenz $\nu_{g}$ existiert, oberhalb derer die Übertragungsfunktion des Messgerätes verschwindet und die höherfrequenten Anteile verloren gehen. Dies bedeutet wiederum eine Beschränkung in der Auflösung im Ortsraum, bei Bildern also etwa der Genauigkeit der räumlichen Abtastung, darstellbar durch die Dichte der Pixel am CCD-Detektor.

Unterschiedlich große Strukturen werden mit verschiedenen Raumfrequenzen identifiziert, wobei die Fourierphasen die Position der Strukturen im Bild kodieren. Bei der Bildentstehung geht die Information über die Phasen der Lichtwellen am Detektor durch Intensitätsbildung verloren, macht sich aber durch Interferenzerscheinungen bemerkbar. Die Phasen des idealen Bildes an verschiedenen Punkten der Bildebene werden durch die Atmosphäre gestört und ergeben deshalb ein verzerrtes Abbild der Lichtquelle. In der vorliegenden Arbeit werden nun zwei Ansätze benutzt, die in die Fourierspektren eingreifen: einerseits die Adaptive Optik, die noch vor dem Verlorengehen der Phaseninformation diese selbst korrigiert bzw. verändert. Bei der Speckle-Interferometrie wird dagegen ausgenutzt, dass gewisse Phasenbeziehungen zwischen verschiedenen Bildpunkten auch nach Durchlaufen der turbulenten Atmosphäre erhalten bleiben, und die Methode ist in der Lage, den 'Originalzustand' dieser Beziehungen, und damit auch die Phasen selbst, wiederherzustellen.

Aus diesen Gründen soll hier auf die wichtigsten Eigenschaften von $\mathcal{F}$ eingegangen werden: Für eine beliebige spektrale Zerlegung benötigt man ein vollständiges System von Basisfunktionen, nach denen die betreffende Funktion entwickelt wird. Speziell bei der Fourier-Transformation dient die komplexe Exponentialfunktion $e^{-i 2 \pi \nu t}=e^{-i \omega t}$ als Basis. Dabei kann $t$ mit der Zeit und $\omega$ mit der zugehörigen Kreisfrequenz identifiziert werden. $\mathcal{F}$ hat die größte Symmetrie, wenn wir die konjugierten Variablen $t$ und $\nu$ benutzen. Bei Verwendung von $\omega$ ergibt sich wegen $d \omega=2 \pi d \nu$ jedoch nur ein konstanter Vorfaktor. Der negative Exponentialkern kennzeichne außerdem die Transformation aus dem Ortsraum in den Frequenzraum. Erfüllt die Funktion $f(t)$ noch gewisse Bedingungen 
(siehe Bracewell (1986)), dann ist ihre Fouriertransformierte $F(\nu)$ definiert durch

$$
F(\nu)=\mathcal{F}\{f(t)\}:=\int_{-\infty}^{+\infty} f(t) e^{-i 2 \pi \nu t} d t
$$

und die Rücktransformation $\mathcal{F}^{+}$ist dann gegeben durch:

$$
f(t)=\mathcal{F}^{+}\{F(\nu)\}=\int_{-\infty}^{+\infty} F(\nu) e^{+i 2 \pi \nu t} d \nu
$$

Die Verallgemeinerung auf höhere Dimensionen geschieht durch die Verwendung des Skalarproduktes. Im zweidimensionalen Raum haben wir dann folgende Form der Transformation, die wir für die Behandlung von Bilddaten benötigen:

$$
\mathcal{F}\{f(x, y)\}=\int_{-\infty}^{+\infty} f(x, y) e^{-i 2 \pi\left(\nu_{x} x+\nu_{y} y\right)} d x d y=F\left(\nu_{x}, \nu_{y}\right)
$$

wobei hier $x$ und $\nu_{x}$ (bzw. $y$ und $\nu_{y}$ ) als konjugierte Variablen verwendet werden.

So, wie komplexe Zahlen in der Gauß'schen Zahlenebene als Vektor mit Betrag und Winkel zur reellen Achse dargestellt werden können, ist das komplexe Fourierspektrum gewöhnlich durch zwei reelle Spektren gegeben: Das power spectrum (PS), also die spektrale Leistungsdichte, ist das Betragsquadrat der Fouriertransformierten,

$$
P S_{f}(\nu)=|\mathcal{F}\{f(t)\}|^{2}=|F(\nu)|^{2}
$$

und die Winkel sind im zugehörigen Phasenspektrum $\Phi_{f}$ enthalten:

$$
\Phi_{f}(\nu)=\arctan \frac{\operatorname{Im}\{F(\nu)\}}{\operatorname{Re}\{F(\nu)\}}
$$

Dabei ist der gesamte Informationsgehalt der Funktionen $f(t)$ und $F(\nu)$ identisch.

Fast Fourier Transform Zur digitalen Datenanalyse mittels Computer muss natürlich die diskrete Form DFT (Digitale Fouriertransformation) für die FT gefunden werden. Statt eines Integrals mit der kontinuierlichen Variablen $x$ bildet man hier eine Summe über $N$ Stützstellen:

$$
F_{l}=D F T\left\{f_{k}\right\}=\frac{1}{N} \sum_{k=0}^{N-1} f_{k} e^{-2 \pi i \frac{k l}{N}}
$$

mit $l=0, \ldots, N$ als Index im diskretisierten Fourierraum.

Wenn wir die Daten als Spaltenvektoren schreiben,

$$
\vec{f}=\left(\begin{array}{c}
f_{0} \\
\vdots \\
f_{N}
\end{array}\right) \quad \text { und } \quad \vec{F}=\left(\begin{array}{c}
F_{0} \\
\vdots \\
F_{N}
\end{array}\right)
$$


lässt sich das $N$-zeilige Gleichungssystem (A.6) als Matrixgleichung schreiben:

$$
\vec{F}=W^{-} \vec{f} \quad \text { bzw. } \quad \vec{f}=W^{+} \vec{F}
$$

mit

$$
W^{ \pm}=\frac{1}{N}\left(\begin{array}{cccc}
1 & 1 & \cdots & 1 \\
1 & e^{ \pm 2 \pi i \frac{1}{N}} & \cdots & e^{ \pm 2 \pi i \frac{N-1}{N}} \\
\vdots & \vdots & \ddots & \vdots \\
1 & e^{ \pm 2 \pi i \frac{N-1}{N}} & \cdots & e^{ \pm 2 \pi i \frac{(N-1)^{2}}{N}}
\end{array}\right)
$$

Die Matrix $W^{ \pm}$enthält also nur Koeffizienten, die von den Daten unabhängig sind. Die DFT kann nun sehr leicht berechnet werden, wobei jedoch die Anzahl der nötigen Rechenschritte mit $N^{2}$ steigt!

Bei äquidistanten Datensätzen kann man nun bei der sogenannten Fast Fourier Transform $(F F T)$ die Matrix $W$ rekursiv in ein Produkt von Matrizen zerlegen, die sehr schnell berechenbar sind. Mit $N=2^{k}$ ergeben sich bei der Zerlegung folgende Untermatrizen:

$$
W_{2^{k}}=\left(\begin{array}{cc}
I_{2^{k-1}} & D_{2^{k-1}} \\
I_{2^{k-1}} & -D_{2^{k-1}}
\end{array}\right)\left(\begin{array}{cc}
W_{2^{k-1}} & 0 \\
0 & W_{2^{k-1}}
\end{array}\right)\left(\begin{array}{c}
\text { Ordner }- \\
\text { matrix }
\end{array}\right)
$$

Die erste Matrix enthält vier Untermatrizen, die alle nur schwach besetzt sind. Bis auf die Hauptdiagonalen sind alle Elemente gleich Null: Es bedeutet $I$ die Einheitsmatrix und $D$ eine Diagonalmatrix mit Exponentialkoeffizienten. Mit $N=8$ bzw. $k=3$ und $\omega=e^{2 \pi i / 2^{N}}$ erhalten wir beispielsweise

$$
I_{2^{k-1}}=\left(\begin{array}{cccc}
1 & 0 & 0 & 0 \\
0 & 1 & 0 & 0 \\
0 & 0 & 1 & 0 \\
0 & 0 & 0 & 1
\end{array}\right) \quad \text { und } \quad D_{2^{k-1}}=\left(\begin{array}{cccc}
\omega^{0} & 0 & 0 & 0 \\
0 & \omega^{1} & 0 & 0 \\
0 & 0 & \omega^{2} & 0 \\
0 & 0 & 0 & \omega^{3}
\end{array}\right)
$$

Die Untermatrizen $W_{2^{k-1}}$ in Gleichung A.10 besitzen nur noch die Hälfte der Spalten- und Zeilenanzahl der Ausgangsmatrix und können wiederum nach der gleichen Vorschrift reduziert werden. Die dritte Matrix verändert schließlich nur die Anordnung der Elemente.

Insgesamt reduziert sich der Rechenaufwand durch die Faktorisierung jedesmal um etwa die Hälfte. Bei der Transformation von Bildern der Größe $N=1024=2^{k}$ ergeben sich durch die $k=10$ Faktorisierungen statt etwa einer Million Rechnungen nur noch etwa 11000. Dieser Umstand macht die FFT zu einem mächtigen Hilfsfmittel, das mit Hilfe der Computer schnelle Bildverarbeitung praktisch erst möglich macht. 



\section{B Der Satz von Wiener-Khinchine}

Die Faltung ' $\otimes$ ' zweier Funktionen $f$ und $g$ ist definiert durch

$$
h(x)=f(x) \otimes g(x)=\int_{-\infty}^{+\infty} f(\xi) g(x-\xi) d \xi
$$

Diese lineare Operation wird zur Beschreibung vieler physikalischer Systeme herangezogen. Wenn ein solches auf das Eingabesignal $f$ mit der Antwort $h$ reagiert, dann beschreibt $g$ die Übertragungseigenschaften des jeweiligen Systems. Die Transformierte $G=\mathcal{F}\{g\}$ heißt Übertragungsfunktion bzw. Transferfunktion.

Ähnlich liefert die Korrelation ' $\star$ ' die Kreuzkovarianzfunktion KKF:

$$
K K F_{f g}(x)=f(x) \star g(x)=\int_{-\infty}^{+\infty} f^{\star}(\xi) g(x+\xi) d \xi
$$

wobei $f^{\star}$ konjugiert komplex zu $f$ ist.

Die $K K F$ gibt an, wie eng zwei Funktionen miteinander zusammenhängen. Das Maximum bezeichnet dann diejenige relative Verschiebung $x$, bei der die größte Übereinstimmung gefunden wird. Die Korrelation einer Funktion $f$ mit sich selbst (Autokorrelation) ergibt analog die Autokovarianzfunktion AKF.

Werden nun diese Integrale fouriertransformiert, wird der einfache Zusammenhang der Funktionen im Frequenzraum sichtbar:

$$
\begin{aligned}
& \mathcal{F}\{f \otimes g\}=F \cdot G \\
& \mathcal{F}\{f \star g\}=F^{\star} \cdot G
\end{aligned}
$$

Insbesondere gilt der Satz von Wiener-Khinchine über die Autokorrelation:

$$
F T\{f \star f\}=F^{\star} \cdot F=|F|^{2}
$$

Um diesen Zusammenhang anschaulich zu machen, mag die graphische Darstellung in Abbildung B.1 behilflich sein. 


\section{Ortsraum $(\mathrm{x}, \mathrm{y})$}

Frequenzraum $\left(v_{x}, v_{y}\right)$

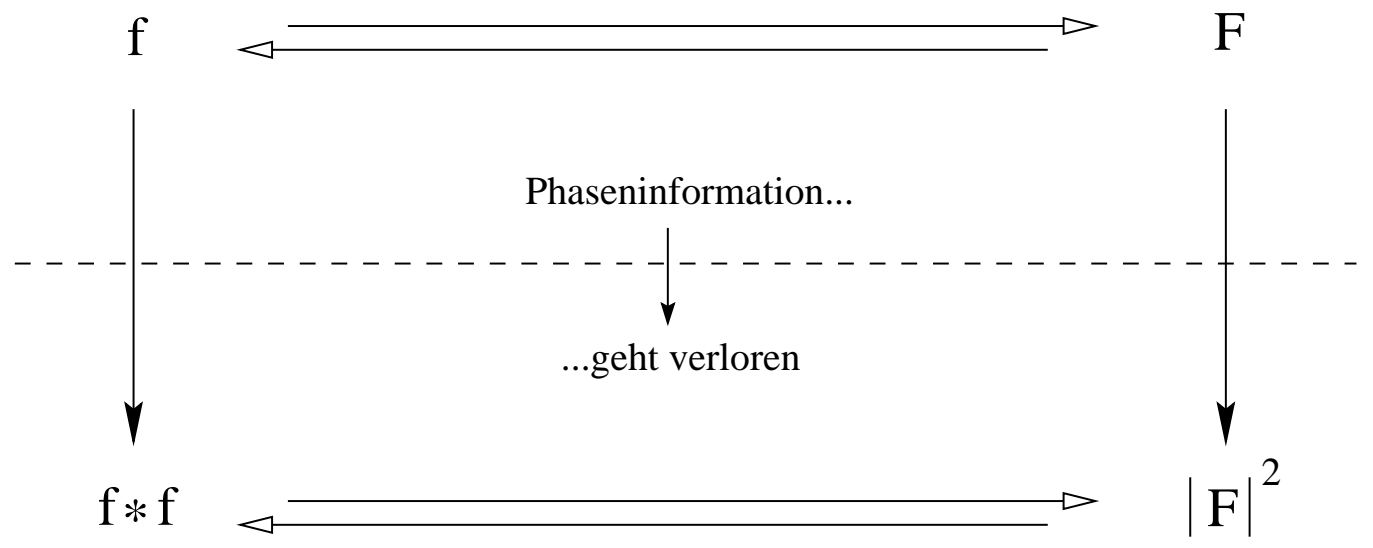

$\mathrm{AKF}$

PS

Abbildung B.1: Zum Wiener-Khinchine-Theorem, das den Zusammenhang zwischen Fourier-Transformation und Autokorrelation beschreibt. Der Übergang vom Ortsraum in den Fourierraum ist umkehrbar. Bei der Bildung der Autokorrelationsfunktion $(A K F)$ oder des Leistungsspektrums $(P S)$ geht jedoch in irreversibler Weise die Phaseninformation verloren. 


\section{Literaturverzeichnis}

Acton, D. S. \& Smithson, R. C., 1992, Solar imaging with a segmented adaptive mirror, Appl. Opt. 31, 3161-3169

Babcock, H., 1953, The possibility of compensating astronomical seeing, Publ. Astron. Soc. Pac. 65, 229-236

Beckers, J. M., 1993, Adaptive optics for Astronomy: principles, performance and applications, Ann. Rev. Astron. Astrophys. 31, 13-62

Berkefeld, T.: Untersuchungen zur Messung und Korrektur einzelner Schichten der Erdatmosphäre, Inaugural-Dissertation, Ruprecht-Karls-Universität, Heidelberg, 1998

Berkefeld, T., Soltau, D., von der LÃijhe, O., 2005, Results of the multi-conjugate adaptive optics system at the German solar telescope, Tenerife. In: Astronomical Adaptive Optics Systems and Applications II, Proc. SPIE 5903, 219-226

Beyer, J. T., Roggemann, M. C., Otten, L. J., Schulz, T. J., Havens, T. C., Brown, W. W., 2003, Experimental estimation of the spatial statistics of turbulence-induced index of refraction fluctuations in the upper atmosphere, Appl. Optics 42, 908-921

Born, M., Wolf, E.: Principles of Optics, Cambridge University Press, 7th edition, 1999

Bracewell, R. N.: The Fourier Transform and Its Applications, $2^{\text {nd }}$ edition (revised), McGraw-Hill, New York, 1986

Brigham, E. O.: FFT - Schnelle Fourier-Transformation, 3. verb. Auflage, R. Oldenbourg Verlag, München, 1987

Cagigal, M. P., Canales, F. V., 2000, Generalized Fried parameter after adaptive optics partial wave-front compensation, Opt. Soc. Am. A, 17, 903-910

Cagigal, M. P., Canales, F. V., 2000, Residual phase variance in partial correction: application to the estimate of the light intensity statistics, Opt. Soc. Am. A, 17, 1312-1318

Coulman, C. E., Vernin, J., Coqueugniot, Y. and Caccia, J. L., 1988, Outer scale of turbulence appropriate to modeling refractive index structure profiles, Appl. Optics 27, $155-160$

de Boer, C. R., \& Kneer, F., 1992, Speckle observations of abnormal solar granulation, Astron. Astrophys. 264, L24-L26 
de Boer, C. R.: Speckle-Interferometrie und ihre Anwendung auf die Sonnenbeobachtung, Dissertation, Georg-August-Universität, Göttingen, 1993

de Boer, C. R., 1996, Noise filtering in solar speckle masking reconstructions, Astron. Astrophys. Suppl. Ser. 120, 195-199

Fried, D. L., 1965, Statistics of a geometric representation of wavefront distortion, J. Opt. Soc. Am. 55, 1427-1435

Fried, D. L., 1966a, Optical resolution through a randomly inhomogeneous medium for very long and very short exposures, J. Opt. Soc. Am. 56, 1372-1379

Fried, D. L., 1966b, Limiting resolution looking down through the atmosphere, J. Opt. Soc. Am. 56, 1380-1384

Fried, D. L., 1982, Anisoplanatism in adaptive optics, J. Opt. Soc. Am. 72, 52-61

Fried, D. L., 1990, Time-delay-induced mean-square error in adaptive optics, J. Opt. Soc. Am. A 7, 1224-1225

Fried, D. L., Atmospheric turbulence optical effects: understanding the adaptive optics implications, in: Alloin, E. M., Mariotti, J.-M., (eds.): Adaptive Optics for Astronomy, NATO ASI Series C 423, 25-57, Kluwer Academic Publishers, Dordrecht, 1993

Fusco, T., \& Conan, J.-M., 2004, On- and off-axis statistical behavior of adaptive-opticscorrected short-exposure Strehl ratio, J. Opt. Soc. Am. A 21, 1277-1289

Glindemann, A., Lane, R. G., Dainty, J. C., 1993, Simulation of time-evolving speckle patterns using Kolmogorov statistics, J. Mod. Opt. 40, Nr. 12, 2381-2388

Greenwood, D. P., 1976, Power spectra requirements for wave-front-compensative systems, J. Opt. Soc. Am. 66, 193

Greenwood, D. P., 1977, Bandwidth specification for adaptive optics systems, J. Opt. Soc. Am. 67, 174-176.

Hardy, J. W.: Adaptive Optics for Astronomical Telescopes, Oxford series in optical and imaging sciences 16, Oxford University Press, New York, 1998

Hartmann, J. F., 1904, Objektivuntersuchungen, Zeitschrift für Instrumentenkunde 24, $1-21$

Knox, K. T., Thompson, B. J., 1974, Astrophysical Journal 193, L45-L58

Kolmogorov, A. N., 1941, Die Energiedissipation für lokal isotrope Turbulenz, Ber. Akad. Wiss. UdSSR, Bd. 32, Nr. 1 (dt. Übersetzung in: Goering, H., (ed.): Sammelband zur statistischen Theorie der Turbulenz, Akademie-Verlag, Berlin, 1958.) (engl.: Dissipation of energy in locally isotropic turbulence, Doklady Akad. Nauk SSSR 32, 16. in: Friedlander, S. K., \& Topper, L., (eds.): Turbulence, Classic Papers on Statistical Theo$r y$, Interscience, New York, 1961) 
Korff, D., 1973, Analysis of a method for obtaining near-diffraction-limited information in the presence of atmospheric turbulence, J. Opt. Soc. Am. 63, 971-980

Labeyrie, A., 1970, Attainment of Diffraction Limited Resolution in Large Telescopes by Fourier Analysing Speckle Patterns in Star Images, Astron. Astrophys. 6, 85-87

Lane, R. G., Glindemann, A., \& Dainty, J. C., 1992, Simulation of a Kolmogorov phase screen, Waves in Random Media 2, 209-224

Lohmann, A. W., Weigelt, G., \& Wirnitzer, B., 1983, Speckle masking in astronomy: triple correlation theory and applications, Appl. Opt. 22, 4028-4037

von der Lühe, O., 1984, Estimating Fried's parameter from a time series of an arbitrary resolved object imaged through atmospheric turbulence, J. Opt. Soc. Am. A 1, 510-519

von der Lühe, O., 1985, The Speckle Masking Transfer Function, Astron. Astrophys. 150, 229-231

von der Lühe, O., 1993, Speckle imaging of solar small scale structure: I. Methods, Astron. Astrophys. 268, 374-390

von der Lühe, O., Berkefeld, T., Soltau, D., Adaptive optics system for a $1.5 \mathrm{~m}$ solar telescope, in: Kohnle, A., Gonglewski, J.D., Schmugge, T.J., (eds.): Optics in Atmospheric Propagation and Adaptive Systems IV, SPIE 4538 (2002), 197-204

von der Lühe, O., Berkefeld, T., Soltau, D., 2005, Multi-conjugate solar adaptive optics at the Vacuum Tower Telescope on Tenerife, Comptes Rendus Physique 6, Issue 10, $1139-1147$

Michau, V., Rousset, G., \& Fontanella, J. C., Wave front sensing from extended sources, in: Radick, R. R., (ed.): Real time and Post-facto Solar Image Correction, NSO/SP Summer Workshop Series No. 13, 124-128, National Solar Observatory, Sacramento Peak, 1992

Mikurda, K., Tritschler, A., \& Schmidt, W., 2006, The influence of image reconstruction on two-dimensional spectrograms of the solar photosphere, Astron. Astrophys., (akzeptiert 28/04/06)

Noll, R. J., 1976, Zernike polynomials and atmospheric turbulence, J. Opt. Soc. Am. 66, 207-211

Obukhov, A. M., 1949, Structure of the temperature field in a turbulent flow, Izv. Akad. Nauk SSSR, Ser. Geograf. Geofiz. 13, 58

Puschmann, K. G., \& Sailer, M., 2006, Speckle reconstruction of photometric data observed with adaptive optics, Astron. Astrophys., (akzeptiert 03/04/2006)

Ragazzoni, R., \& Farinato, J., 1999, Sensitivity of a pyramidic wave front sensor in closed loop adaptive optics, Astron. Astrophys. 350, L23-L26 
Roddier, F., The Effects of Atmospheric Turbulence in Optical Astronomy, in: Wolf, E., (ed.), Progress in Optics XIX, 283-376, North-Holland, New York, 1981

Roddier, F., 1988, Curvature sensing and compensation: a new concept in adaptive optics, Appl. Opt. 27, 1223-1225

Roddier, F., Northcott, M., Graves, J., McKenna, D., \& Roddier, D., 1993, Onedimensional spectra of turbulence-induced Zernike aberrations: time-delay and isoplanicity error in partial adaptive compensation, J. Opt. Soc. Am. A 10, 957-965

Roddier, F.: Adaptive Optics in Astronomy, Cambridge University Press, 1999

Roggemann, M. C., Welsh, B. M.: Imaging Through Turbulence, CRC Press, Boca Raton, Fla., 1996

Rousset, G., Wave front sensing, in: Alloin, E. M., Mariotti, J.-M., (eds.): Adaptive Optics for Astronomy, NATO ASI Series C 423, 115-137, Kluwer Academic Publishers, Dordrecht, 1993

Sailer, M.: Speckle-Rekonstruktion von großformatigen Bildserien solarer Feinstrukturen, Diplomarbeit, Georg-August-Universität, Göttingen, 2002

Sasiela, R. J. \& Mooney, J. G., 1985, An optical phase reconstructor based on using a multiplier-accumulator approach, Proc. SPIE 551, 1088-1098.

Scharmer, G. B., Bjelksjö, K., Korhonen, T. K., Lindberg, B. \& Petterson, B., 2003a, The 1-meter Swedish solar telescope, Proc. SPIE 4853, 341-350

Scharmer, G. B., Dettori, P. M., Löfdahl, M. G. \& Shand, M., 2003b, Adaptive optics system for the new Swedish solar telescope, Proc. SPIE 4853, 370-380

Séchaud, M., Wave-front compensation devices, in: Roddier, F.: Adaptive optics in astronomy, Cambridge University Press, 1999

Shack, R. B. \& Platt, B. C., 1971, Production and use of a lenticular Hartmann Screen (abstract), J. Opt. Soc. Am. 61, 656

Strehl, K., 1902, Über Luftschlieren und Zonenfehler, Zeitschrift für Instrumentenkunde 22, 213-217

Tatarskij, V. I.: Wave Propagation in a Turbulent Medium, McGraw-Hill, New York, 1961

Thiébaut, E., Introduction to image reconstruction and inverse Problems, in: Foy, R., \& Foy, F. C., (eds.): Optics in Astrophysics, NATO ASI Science Series II, Vol. 198, $397-$ 421, Springer, 2005

Valley, G. C., 1980, Isoplanatic degradation of tilt correction and short-term imaging systems, Appl. Opt.19, 574-577

Wang, J. Y. \& Markey, J. K., 1978, Modal compensation of atmospheric turbulence distortion, J. Opt. Soc. Am. 68, 78-87 
Weigelt, G. P., 1977, Modified astronomical Speckle interferometry "Speckle masking", Opt. Commun. 21, Nummer 1, 55-59

Weigelt, G. P., Wirnitzer, B., 1983, Image Reconstruction by the Speckle masking method, Opt. Letters 8, 389-391

Weiß, A. R.: Point Spread Function Reconstruction for the Adaptive Optics System ALFA and its Application to Photometry, Dissertation, Ruprecht-Karls-Universität, Heidelberg, 2003

Winker, D., 1991, Effect of a finite outer scale on the Zernike decomposition of atmospheric optical turbulence, J. Opt. Soc. Am. A 8, 1568-1573

Wöger, F.: Diagnostik der Korrekturleistung adaptiver Optik bei der Sonnenbeobachtung, Staatsexamensarbeit, Albert-Ludwigs-Universität, Freiburg i. Brsg., 2001 



\section{Danksagung}

Nach vielen Jahren erfahrener Betreuung, immer wiederkehrender Motivierung und kollegialer Freundschaft freut es mich, zu dieser Gelegenheit meiner Dankbarkeit gegenüber meinem Doktorvater Prof. Dr. Franz Kneer Ausdruck verleihen zu können! Franz, ein herzliches 'Vergelt's Gott', wie man in meiner Heimat im Allgäu sagt! Ich danke Dir sehr für alle Förderung, die ich durch Dich immer wieder erfahren habe, für die außerordentlich freundliche Arbeitsatmosphäre und für Dein persönliches Begleiten meiner Ausbildung in den letzten sieben Jahren - das war mein Auslandsjahr auf Teneriffa, die anschließende Diplomarbeit in der Sternwarte und die Zeit als Doktorand an der alten Göttinger Sternwarte und danach hier im neuen Institut für Astrophysik.

Am Kiepenheuer-Institut für Sonnenphysik (KIS) in Freiburg möchte ich ganz besonders einem weiteren Begleiter meiner Arbeit danken, Prof. Dr. Oskar von der Lühe! Ohne seinen sprühenden Enthusiasmus, seinen Ideenreichtum mit Tipps und Tricks, und sein außergewöhnliches didaktisches Talent zusammen mit dem ungeheuren Fachwissen, hätte ich wohl nicht promoviert und längst das Weite gesucht! Herr von der Lühe, Sie hatten trotz aller anderen Verpflichtungen immer auch ein Ohr für meine Angelegenheiten und Schwierigkeiten und halfen mir recht oft wieder auf die Beine! Auch wenn es nicht einfach ist, 'in Ihre Fußstapfen zu treten', so hoffe ich trotzdem auf intensiven fachlichen Austausch und weiterhin freundschaftlichen Kontakt! Vielen herzlichen Dank!

In meiner Arbeitsgruppe und meinem Heimat-Institut 'Sternwarte Göttingen' möchte ich besonders Nazaret Bello González für die vielen Jahre freundlichen Arbeitsklimas, die fachlichen Diskussionen und nicht zuletzt für die angenehme Atmosphäre von Fürsorglichkeit danken. Klaus Puschmann half mir außerordentlich, in das wissenschaftliche Leben aktiv einzusteigen, was nicht einfach war! Bruno Sánchez-Andrade Nuño, Julian Blanco Rodriguez, Klaus Reinsch und Wilfried Steinhof danke ich sehr für ihre Kollegialität und die technischen Hilfen in Notsituationen! Gedankt sei ehemalige Kollegen an der Sternwarte, Moritz Kirschmann, Oleg Okunev, Itahiza Domínguez Cerdeña, Aleksandra Andjic, Katja Janßen und Maren Wunnenberg.

Am KIS in Freiburg arbeiten eine Vielzahl von Leuten, denen ich gerne danken möchte: Meinem Kollegen Friedrich Wöger, der mir seit den Tagen der Diplomarbeit ein guter Freund geworden ist und mir immer wieder mit Rat und Tat zur Seite stand und mir immer bereitwillig Unterkunft gewährte, obwohl das für seine junge Familie sicher auch eine Belastung war. Friedrich, vielen Dank deshalb auch an Deine Frau Amanda!

Thomas Berkefeld danke ich für seine Hilfen zum Verständnis der Adaptiven Optik und die gute Atmosphäre am Teleskop!

Sven Bingert danke ich für einen schönen Sommer, besonders für die harte Heraus- 
forderung am Klavier - vielleicht lern' ich doch noch einmal das vom-Blatt-spielen?

Viele Diskussionen konnte ich mit Christian Beck führen, der sich immer auf meine fachlichen Probleme einließ und immer konstruktive Fragen oder Vorschläge machen konnte. Christian, am liebsten erinnere ich mich aber vor allem an die geistreiche und witzige Arbeitsatmosphäre mit Dir zurück!

Alexandra Tritschler danke ich besonders, für viel seelische Unterstützung in allen stressigen Aspekten einer Doktorarbeit!

Ein herzliches Dankeschön an Rolf Schlichenmaier, der mich immer wieder auch etwas wachrüttelte und Haltung annehmen ließ

Vielen Dank an das KIS-Kino-Team, Tayeb Aiouaz, Petri Käpylä, Mathieu Ossendrijver, Dirk Schmidt, Pia Zacharias, Stefan Jendersie, Christof Prahl, Reza Rezaei, Lars Krieger, Kasia Mikurda, Sven Wedemeyer-Böhm, Oskar Steiner, Peter Caligari, Dirk Soltau und Frau Gertrud Abadia!

Aus dem technischen Bereich danke ich Robert Fellmann, Thomas Keller, Oliver Wiloth und Thomas Hederer, mit denen ich ein ausgesprochen kollegiales Klima genossen habe - auf Teneriffa wie in Freiburg!

Am Max-Planck-Institut für Sonnensystemforschung (MPS) sind bzw. waren sehr viele Gefährten auf dem Weg zur Promotion im Rahmen der International Max Planck Research School (IMPRS) tätig. Die jährlichen 'Retreat'-Wochen, Vorlesungen und Seminare haben den Kontakt und den fachlichen Horizont ungemein verbreitert.

Besonders seien erwähnt: Chun Ming Mark Cheung, Sabine Preusse, María Hebe Cremades Fernández, Denise Tortorella, Tra-Mi Ho, Emre Isik, Santo Salinas, Lidong Xia, Marilena Mierla, Vasily Zakharov, Durgesh Kumar Tripathi, Rupali Arunkumar Mahajan, Aveek Sarkar, Dragos Ovidiu Constantinescu, Martin Schrinner, Alexander Vögler, Lotfi Yelles Chaouche, Lucas Paganini, Sergey I. Shelyag, Lucasz Matloch, Ganna Portyankina, Ana Teresa Monteiro Tomás, Michael Heuer, Monika Buske, Ingo Jens Baumann, Juan Manuel Borrero Santiago, Martin Tschimmel, Redouane Mecheri, Clementina Sasso und Danica Tothova.

In Kanada danke ich Laurent Jolissaint und Jean-Pierre Véran am NRC Herzberg Institute of Astrophysics (NRC-HIA) in Victoria, B. C., für die Möglichkeit, meine Arbeit einem internationalen Publikum vorzustellen. In diesem Zusammenhang sei den MitOrganisatoren Julian Christou am Center for Adaptive Optics in Santa Cruz, Kalifornien, und Thomas Rimmele vom National Solar Observatory (NSO) in New Mexiko gedankt. Ich danke Jeffrey A. Stösz am Dominian Astrophysical Observatory (DAO), ebenfalls Victoria, B. C. , für interessante Diskussionen bei der Summer school in Cargése, Korsi$\mathrm{ka}$, und in Victoria beim Workshop zur AO-PSF!

Außerdem geht mein Dank an Kai Morgenstern und Kerstin Hewel in Vancouver! Vielen Dank für die Gastfreundschaft und Unterstützung. Auf intensiven weiteren Kontakt!

Für die finanzielle Unterstützung, ohne die diese Arbeit nicht entstanden wäre, bin ich folgenden Stellen sehr dankbar:

Der Deutschen Forschungsgemeinschaft, die die Arbeit in den ersten 2 1/2 Jahren als Träger des Graduiertenkollegs 140 'Strömungsinstabilitäten und Turbulenz' gefördert hat. Dem Kiepenheuer-Institut für Sonnenphysik (KIS) in Freiburg, bei dem ich während ei- 
nes halben Jahres unter Vertrag intensive Forschung treiben konnte und dabei Gelegenheit hatte, wertvolle fachliche und persönliche Kontakte aufzubauen und zu pflegen.

Dem Institut für Astrophysik (IAG) in Göttingen und dem Max-Planck-Institut für Sonnensystemforschung in Katlenburg-Lindau, die mir in der Endphase meiner Arbeit durch die finanziellen Mittel die Möglichkeit gaben, mich voll auf die Dissertation zu konzentrieren. Hier danke ich besonders Prof. Dr. Franz Kneer und PD Dr. Dieter Schmitt!

Außerdem möchte ich mich in diesem Zusammenhang noch besonders bei meinem Gönner und Förderer H. H. Geistl. Rat Rudolf Sinz in Weiler im Allgäu bedanken! Herzliches 'Vergelt's Gott' für Deine stille Unterstützung während der letzten Jahre und, an dieser Stelle besonders zu betonen, für alle Hilfen seit meinen Tagen im Internat in St. Ottilien. Vielen herzlichen Dank!

Allen Freundinnen und Freunden möchte ich gerne danken, die mich in den vergangenen Jahren der Doktorarbeit begleitet haben: Helmut Hölzler, Elke Wiludda, Vivien Kurtz, Naomi Fretz, Juliette Anglehart, Naomi Crey, Arnélida Gorrin, Meike Wollni, Robert Mettin, Julien Aubert, Malte Klar, Verina Wild, Nadja Reusch, Karin Raiser, das ganze Spanisch-Cafe, Ursula Köberle, Petra Maaß Jasivia Gonzales, Regina Koch, Antje Seeber, Malte Jitschin, Sven Anderson, Gunnar Heunisch, Martin Laging, Nils Heunisch, Olaf Cohrs, Tina Kruse, Rüdiger Hartwich, Ester Schoppe, Sabine Hüttl, Regina Günther, Stephanie Gümbel, Sabine, Stephan Dongus, Tom Tetzlaff, Tanja Faust, Heiko Rode und Martha Decker.

Besonders möchte ich meiner lieben Joana Krause danken, die mich gerade im letzten Jahr immer wieder mal aushalten musste und mich wieder aufrichtete. Ich freue mich auf unseren weiteren Weg!

Schließlich möchte ich meinen Eltern danken, die mir die Möglichkeiten gaben, diesen Bildungsweg einzuschlagen, mich immer persönlich und finanziell unterstützten und mir immer ein warmes Willkommen zu Hause bereiten. Herzliches Vergelt's Gott! 



\title{
Lebenslauf
}

\author{
Name: $\quad$ Markus Josef Sailer \\ Geburt: $\quad$ 12. März 1974 \\ Geburtsort: Kempten im Allgäu \\ Staatsangehörigkeit: deutsch \\ Eltern: \\ Anselm Sailer und Hildegard Sailer (geb. Keller) \\ 09/1980 - 07/1984 Grundschule Heiligkreuz, Kempten \\ 09/1984 - 07/1986 Carl-von-Linde Gymnasium, Kempten i. Allg. \\ 09/1986 - 07/1993 Rhabanus-Maurus Gymnasium, St. Ottilien (Internat) \\ 07/1993 Abitur \\ 11/1993 - 01/1995 Zivildienst, Alten- und Pflegeheim, Grafrath (Fürstenfeldbruck) \\ 10/1995 - 05/2002 Studium der Physik (Diplom) \\ an der Georg-August-Universität Göttingen \\ 10/1999 - 09/2000 Auslands-Studienaufenthalt \\ an der Universidad de La Laguna, Teneriffa (Spanien) \\ 03/2000 - 08/2000 Diplompraktikum mit Abschlussarbeit: "Espectropolarimetría solar" \\ 1/2001 - 03/2002 Diplomarbeit an der Göttinger Sternwarte: \\ "Speckle-Rekonstruktion von großformatigen Bildserien \\ solarer Feinstrukturen" \\ $05 / 2002$ \\ Diplom mit Abschluss "sehr gut" \\ 09/2002-07/2006 Doktorarbeit an der Sternwarte Göttingen \\ bzw. am Institut für Astrophysik Göttingen (IAG): \\ "Simulationsrechnungen anisoplanatischer Übertragungsfunktionen \\ für solare Adaptive Optik" \\ 04/2005 - 09/2005 Forschungsaufenthalt im Rahmen der Dissertation \\ am Kiepenheuer-Institut für Sonnenphysik (KIS) in Freiburg
}

